\title{
Magnetic nanoparticles studied by synchrotron radiation and rf transverse susceptibility
}

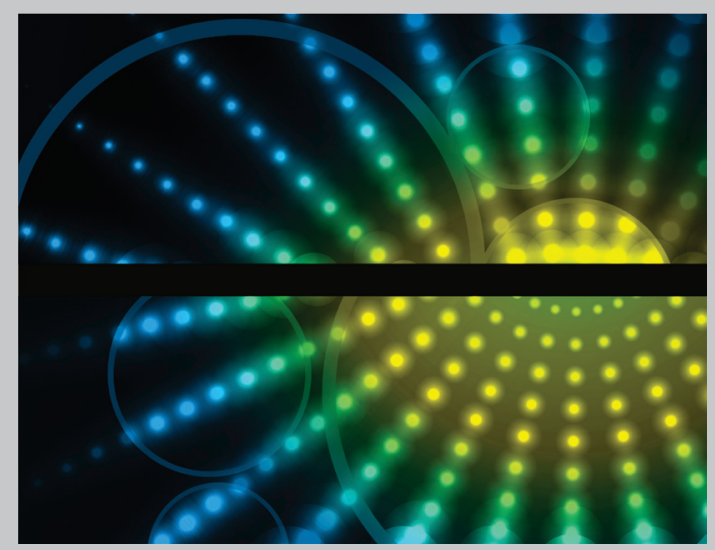

Adriana Isabel Figueroa García 

Magnetic nanoparticles studied by synchrotron radiation and rf transverse susceptibility 


\section{Colección de Estudios de Física}

\section{Vol. 103}

Esta colección recoge las tesis presentadas en el Departamento de Física de la Materia Condensada de la Universidad de Zaragoza desde su constitución en 1987. 
Colección de Estudios de Física

Vol. 103

\section{Magnetic nanoparticles studied by synchrotron radiation and rf transverse susceptibility}

Adriana Isabel Figueroa García

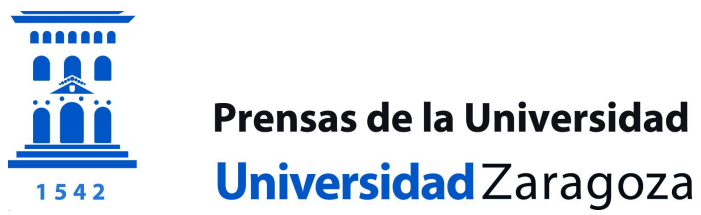




\section{FIGUEROA GARCÍA, Adriana Isabel}

Magnetic nanoparticles studied by synchroton radiation and rf transverse susceptibility / Adriana Isabel Figueroa García. — Zaragoza : Prensas de la Universidad de Zaragoza, 2013

XI, 181 p. ; 25 cm. - (Colección de estudios de física ; 103)

Bibliografía: p. 165-178. — ISBN 978-84-15770-49-7

1. Materiales magnéticos-Tesis doctorales. 2. Nanotecnología-Tesis doctorales 539.622(043.2)

$620.22(043.2)$

Cualquier forma de reproducción, distribución, comunicación pública o transformación de esta obra solo puede ser realizada con la autorización de sus titulares, salvo excepción prevista por la ley. Diríjase a CEDRO (Centro Español de Derechos Reprográficos, www.cedro.org) si necesita fotocopiar o escanear algún fragmento de esta obra.

(C) Adriana Isabel Figueroa García

(C) De la presente edición, Prensas de la Universidad de Zaragoza 1. ${ }^{a}$ edición, 2013

Prensas de la Universidad de Zaragoza. Edificio de Ciencias Geológicas, c/ Pedro Cerbuna, 12, 50009 Zaragoza, España. Tel.: 976761 330. Fax: 976761063

puz@unizar.es http://puz.unizar.es

Impreso en España

Imprime: Servicio de Publicaciones. Universidad de Zaragoza

D.L.: Z 918-2013 
To my dearest family, in loving memory of my dad... 



\section{Contents}

$\begin{array}{ll}\text { Introduction } & 1\end{array}$

1 Experimental techniques $\quad 9$

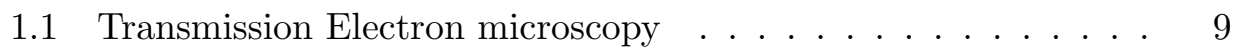

1.1 .1 JEOL JEM $3000 \mathrm{~F} \ldots \ldots \ldots$

1.1 .2 FEI Tecnai ${ }^{\mathrm{TM}}$ G2 F30 STWIN . . . . . . . . . 11

1.2 SQUID based magnetometry $\ldots \ldots \ldots \ldots \ldots$

1.3 Anomalous Hall Effect . . . . . . . . . . . . . . . . . . . . 13

$1.4 \mathrm{X}$-ray absorption spectroscopies in synchrotron radiation facilities 15

1.4.1 X-ray absorption fine structure . . . . . . . . . . 17

1.4.2 X-ray magnetic circular dichroism . . . . . . . . . 21

2 Structural and magnetic properties of amorphous Co-W al$\begin{array}{lr}\text { loyed nanoparticles } & 29\end{array}$

2.1 Samples studied . . . . . . . . . . . . . . . . . . 30

2.2 Morphological and structural study . . . . . . . . . . . . . 32

2.2 .1 Microscopy Measurements . . . . . . . . . . . . . 32

2.2.2 XANES and EXAFS measurements . . . . . . . 39

2.3 Magnetic study . . . . . . . . . . . . . . . 50

2.3.1 SQUID-based magnetometry . . . . . . . . . 50

2.3.2 Magnetic anisotropy of the Co-W particles . . . . . . 55

2.3.3 X-ray Magnetic Circular Dichroism magnetometry . . . 57

2.4 Discussion . . . . . . . . . . . . . . . . . . . 61

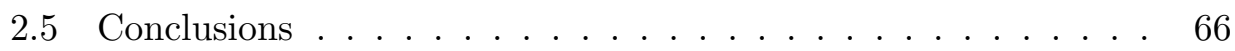

3 Breakdown of Hund's third rule in amorphous Co-W alloy $\begin{array}{ll}\text { nanoparticles } & \mathbf{6 7}\end{array}$

3.1 XANES and XMCD measurements . . . . . . . . . . 68

3.1 .1 Co $K$ edge results . . . . . . . . . . . . . . . . . . . . . 69

$3.1 .2 \quad \mathrm{~W} L_{2,3}$ edges results . . . . . . . . . . . . . 71

3.2 Calculations of DOS, XANES and XMCD . . . . . . . 75

3.2 .1 DOS calculations . . . . . . . . . . 76 
3.2 .2 Co $K$ edge . . . . . . . . . . . . . . . . . . . 77

$3.2 .3 \mathrm{~W} L_{2,3}$ edges . . . . . . . . . . . . . . . . . . . . . . . . . . . . 78

3.3 Discussion ..................... 79

3.4 Conclusion .................... 82

4 Structural and magnetic properties of Co-Pt nanoparticles $\mathbf{8 3}$

4.1 Samples studied . . . . . . . . . . . . . . . . 84

4.2 Morphological and structural study . . . . . . . . . . . . . 84

4.2.1 Microscopy Measurements ............ . 84

4.2.2 XANES and EXAFS measurements . . . . . . . . 87

4.3 Magnetic study .................... 95

4.3.1 SQUID-based magnetometry . . . . . . . . 95

4.3.2 Anomalous Hall Effect magnetometry . . . . . . . . . . 99

4.3.3 X-ray Magnetic Circular Dichroism magnetometry . . . 101

4.4 Discussion . . . . . . . . . . . . . . . . 107

4.5 Conclusions ..................... 109

5 d-band magnetism of $\mathrm{Ag}, \mathrm{Au}, \mathrm{Pd}$ and $\mathrm{Pt}$ nanostructured ma$\begin{array}{ll}\text { terials } & \mathbf{1 1 1}\end{array}$

5.1 Magnetism in bulk metals . . . . . . . . . . . . . . . . 111

5.2 Induced magnetism in nanoparticle matrix . . . . . . . . . . . 113

5.3 Intrinsic magnetic moment in M nanoparticles . . . . . . . . . . 115

5.4 Conclusions .................... 121

6 Transverse susceptibility measurement system for the PPMS123

6.1 Theoretical model of the magnetic transverse susceptibility . . 124

6.2 Description of the TS measurement system . . . . . . . . . . 126

6.3 Design and implementation of the oscillator circuit . . . . . . . 129

6.4 Data acquisition ................... 134

6.5 Test Measurements . . . . . . . . . . . . . . . . . . 135

6.5.1 Test measurements on an empty coil . . . . . . . . 135

6.5.2 Calibration of the TS measurement system with $\mathrm{Gd}_{2} \mathrm{O}_{3} 137$

6.5.3 Test measurements on a known and previously characterized sample with a TDO based TS system . . . . . 138

6.5.4 Low temperature measurements on $\mathrm{TmCo}_{2}$. . . . . . 140

6.6 Conclusions ..................... 141

7 Transverse susceptibility of iron oxide nanoparticles systems 143

7.1 Magnetite Nanoparticles . . . . . . . . . . . . . . . . . . 144

7.1.1 Experimental . . . . . . . . . . . . . 144

7.1.2 Magnetic Properties . . . . . . . . . . . . . 145

7.1.3 Discussion . . . . . . . . . . . . . . . . 149

7.1.4 Conclusions . . . . . . . . . . . . . 152 
7.2 Maghemite Nanoparticles . . . . . . . . . . . . . . 153

7.2 .1 TS measurements . . . . . . . . . . . . . 154

7.2 .2 Discussion . . . . . . . . . . . . . 156

7.2 .3 Conclusions .................. 159

$\begin{array}{ll}\text { General Conclusions } & 161\end{array}$

$\begin{array}{ll}\text { Bibliography } & 165\end{array}$

$\begin{array}{lr}\text { Publications } & 179\end{array}$ 



\section{Introduction}

The fundamental understanding of nanoscale materials has been the focus of much scientific and technological interest over the last decades. Nanoparticles (NPs) are widely investigated not only as consequence of the general miniaturization of devices, but also because they constitute ideal systems to study finite-size and surface effects, those yielding new physical phenomena and enhanced properties with respect to their bulk counterpart $[1-3$.

Magnetic NPs are especially interesting since the properties of the valence electrons of atoms (which are mainly responsible of the magnetic character of atoms and solids) change as they become part of a small particle and start to delocalize. Thus, their magnetic properties are very sensitive to size, composition, and local atomic environment, showing a wide variety of intriguing phenomena. This rich variety of behavior can be understood in general terms as arising from the reduced average coordination per atom, the quantum size effect and modified collective electron behavior such as screening and charge spill-out from the surface $[2]$.

The size of magnetic nanoparticles is comparable to the size of a magnetic domain, which results in a completely novel phenomenology with respect to bulk magnetic materials. Macroscopic magnetic materials reduce their energy by breaking into magnetic domains, or small regions where the spins are aligned. Each domain can have a magnetic moment which is oriented in a different direction from that of its neighbor. The creation of a domain increases the anisotropy energy of the system, defined as the change in the electronic energy, associated to the inversion of its magnetization; but it reduces the magnetostatic energy, so that the material is in average not magnetized (possessing a net magnetization) unless an external magnetic field is applied.

For a particle of radius $R$, the energy of a domain wall is expressed as

$$
E_{p}=4 \sqrt{A K} \pi R^{2}
$$

where $A$ is the exchange interaction and $K$ the anisotropy constant. For 
magnetic particles, there exists a limit of size (critical size) below which it is no longer energetically favorable to create domains in the system. Therefore, it becomes a single-domain particle with a net magnetization. As isolated particles they can be considered as single giant moments of ferromagnetically coupled atomic spins that can be aligned by the application of an external magnetic field. When a saturating field is removed, the magnetization decays with a relaxation rate. This behavior is described by the Arrhenius' law

$$
\tau=\tau_{0} \exp \left(\frac{U}{k_{B} T}\right)
$$

with $k_{B}$ the Boltzmann constant and $T$ the temperature. It describes the time $\tau$ for a particle to overcome an energy barrier $U$ (directly related to the anisotropy energy of each particle), with an attempt frequency $\tau_{0}$. At high temperatures the thermal energy is much greater than the anisotropy energy of each particle, so that all magnetization directions are almost energetically equal. The magnetization is then described by the classical Langevin function $\lfloor 4\rfloor$ as

$$
L(x)=m_{\text {part }}\left(\operatorname{coth}(x)-\frac{1}{x}\right)
$$

with $x=\frac{m_{\text {part }} H}{k_{B} T}, m_{\text {part }}=M_{s} V$ the magnetic moment of a single particle, $M_{s}$ the saturation magnetization, $V$ the particle volume, and $H$ the applied field.

This behavior is known as superparamagnetism. The relaxation time in these systems and their magnetization strongly depend on the temperature. If the net moment of the particle can freely flip from one orientation to the other, the particle is said to be unblocked. On the contrary situation, it is said to be blocked. The temperature that separates both regimes is defined as the blocking temperature $T_{B}$, which depends on the particles intrinsic properties and size.

The distinct behavior of magnetic NPs constitute an important challenge for technical applications, which include diverse fields such as biomedicine $[3$, $5\rfloor$, high-density magnetic recording $\lfloor 2,6,7\rfloor$, and magnetic resonance imaging (MRI) $[8,9$. Each of these areas require of magnetic particles with specific characteristics, for which it is crucial the comprehension and tailoring of their magnetic properties like anisotropy or dipolar interactions between particles. In the hard drive industry, for example, the main objective is to find ways of increasing the magnetic anisotropy of single domain NPs, so that storage densities can continue to increase without compromising the stability of the 
media; this may occur since thermal fluctuations can lead to destabilization of the magnetic state of the particles. On the contrary, biomedical applications need magnetic particles with low anisotropy, for their magnetization to be easily reversed inside biological systems.

Therefore, research in magnetic nanoparticles involves several aspects to consider, from the preparation procedures to the comprehension, control and optimization of the desired properties. For instance, the possibilities of tuning the magnetic anisotropy of the particles in order to fulfill the requirements for each application seem to be of paramount importance. Different experimental approaches for a complete structural and magnetic characterization of the NPs are fundamental to attain this purpose. Following this scheme, in this dissertation we describe different studies performed on various magnetic nanoparticles systems where a proper combination of structural, electronic, and magnetic characterization methods lead to a better understanding of the NPs properties, in particular, of their magnetic anisotropy.

In the first part, we make use of some synchrotron radiation techniques. The unique properties of this radiation such as broad energy spectrum, variable light polarization, and flexible time structure, have made it an enormously powerful tool in the study of magnetic phenomena and structure of materials. Extensive characterization of magnetic particles has been carried out over the last decades with synchrotron based spectroscopic techniques demonstrating their possibilities. We focus here on some X-ray absorption techniques: Xray Absorption near edge structure (XANES), extended X-ray absorption fine structure (EXAFS) and X-ray magnetic Circular Dichroism (XMCD). These techniques constitute very useful methods to directly and selectively examine the electronic state and local structure of the atoms composing the particles, which are fundamental as resulting from the surface effects in reduced size materials. Moreover, the average coordination number, which is also a key parameter that controls the evolution of the magnetic behavior in materials, may be directly determined by EXAFS. On top of that, XMCD arises as an effective method to selectively study the magnetic properties of the particles $\lfloor 2,10-15\rfloor$, giving access to both contributions to the total magnetic moment, the atomic orbital moment $m_{\mathrm{L}}$ (currents associated with the electronic motion) and spin moment $m_{\mathrm{S}}$ (intrinsic angular momentum of the electrons).

The possibility of separating both contributions is especially useful in itinerant magnetic materials, which have an orbital magnetic moment induced by spin-orbit coupling. The general trend in $m_{\mathrm{L}}$ is the same one as for the spin moment: the orbital moment increases with decreasing coordination $\lfloor 2\rfloor$. However, the orbital moment does not linearly follow the spin moment, the ratio $m_{\mathrm{L}} / m_{\mathrm{S}}$ varies with atomic structure, dimensionality and coordination 
number. $m_{\mathrm{L}}$ in NPs is very sensitive to neighboring atoms, increasing rapidly with a decrease in the coordination number, with only the two outer shells of NPs showing values being significantly different from bulk values. This enhancement of $m_{\mathrm{L}}$ and its anisotropy at the NPs surface has been demonstrated to contribute significatively to the total magnetocrystalline anisotropy, as proposed by Bruno [16], following the expression

$$
\Delta E_{\mathrm{SO}} \propto-\xi\left(m_{\mathrm{L}}^{\perp}-m_{\mathrm{L}}^{\|}\right) \propto-K_{\mathrm{S}}
$$

with $\xi$ the spin-orbit coupling of the atoms forming the particle surface, $m_{\mathrm{L}}^{\|}$and $m_{\mathrm{L}}^{\perp}$ the in-plane and out-of-plane components of the orbital moment, respectively, and $K_{\mathrm{S}}$ the surface anisotropy constant. Therefore, the spinorbit coupling in systems of nanometric dimensions has been found to be highly relevant. In this sense, this dissertation continues previous studies of spin-orbit enhanced anisotropy observed in different cobalt NPs systems. They consist of self-organized lattices of nanometer-sized Co magnetic clusters (1-4 nm) prepared by sequential deposition of $\mathrm{Al}_{2} \mathrm{O}_{3}$ and Co layers on a $\mathrm{Si}$ substrate. In these granular systems, it is possible to control the clusters size $\lfloor 17$, the average number of neighbors to a given cluster $\lfloor 18$, and even the surface anisotropy by capping the cobalt nanoparticles with noble metals like $\mathrm{Cu}, \mathrm{Ag}$, $\mathrm{Au}[19]$. Those studies allowed quantitative determination of parameters like magnetic anisotropy $[20,21\rfloor$ or dipolar interactions $[18\rfloor$. In the case of Cometal(M) particles, with $\mathrm{M}=\mathrm{Cu}, \mathrm{Ag}, \mathrm{Au}$, the capping metal does not diffuse on Co, so the Co particle retains its integrity as a nearly spherical crystalline particle. A systematic increase in the surface anisotropy of Co-M NPs with capping in the trend $\mathrm{Cu}, \mathrm{Ag}$, and $\mathrm{Au}$ was then observed, as resulting from the previously described orbital moment anisotropy, caused by hybridization of Co atoms at the particle surface with the capping metal [11].

Beside those surface effects, the magnetic anisotropy of NPs systems may be intrinsic, such as that identified in chemically ordered CoPt alloy NPs $[22$. The anisotropy of those particles is also correlated with the Co orbital anisotropy, which by hybridization with $\mathrm{Pt}$, and due to the high spin-orbit coupling of the latter, gives rise to an enhanced anisotropy, albeit much smaller than in the bulk CoPt $L 1_{0}$ alloy phase $\lfloor 23,24\rfloor$. Therefore, in the Co-M NPs systems that we describe in this thesis, we explore the introduction of high spin-orbit non-magnetic elements and induce their alloying with Co, as a strategy to control their intrinsic anisotropy and magnetic properties. In particular, we study Co NPs capped with Tungsten (W) and Platinum (Pt).

On the one hand, $\mathrm{W}$ (electron configuration $[\mathrm{Xe}] 4 f^{14} 5 d^{4} 6 s^{2}$ ) is a good candidate as it hybridizes with Co (electron configuration $[\mathrm{Ar}] 3 d^{7} 4 s^{2}$ ), its affinity to alloy with Co on sputtering grown films at room temperature is known 
$\lfloor 25\rfloor$, these alloys tend to become amorphous $\lfloor 26-29\rfloor$, and it has a less than half filled $5 d$ band. This way, we end up with a system of amorphous NPs, which constitute a different type with variable anisotropy. Then, the work on Co-W NPs we describe in this dissertation is devoted to investigate the open question whether amorphous alloys may support intrinsic anisotropy, induced by alloying, irrespective of surface effects.

On the other hand, for the Pt case, aside from the previously described hybridization between $\mathrm{Pt}$ (electron configuration [Xe $] 4 f^{14} 5 d^{9} 6 s^{1}$ ) and Co, and the high spin-orbit coupling of $\mathrm{Pt}$ that gives rise to an enhanced anisotropy, Co-Pt NPs show perpendicular magnetic anisotropy (PMA) [30, 31], which is interesting for applications in spintronic nano-devices implemented to, for example, ultrahigh density magnetic storage, fast memory applications, and nanosensors $\lfloor 32,33\rfloor$.

Apart from the importance of the orbital moment in nanomaterials, the spin moment still dominates their magnetism, as in the case of transition metals. Reducing the dimension leads to a narrowing of the valence $d$ bands that generate most of the magnetic moment. The increase in the density of states at the Fermi level $E_{f}$ also opens the chance to detect magnetic phenomena in materials which are non-magnetic in bulk, for example, in the noble metals of

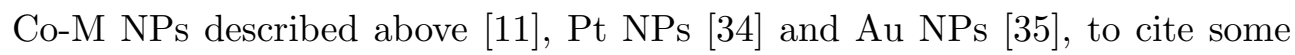
examples of metallic particles where such phenomena have been observed. Accordingly, in this dissertation we present a review of some of these examples as an attempt towards the comprehension of the $d$-band magnetism in nanostructured metals via the element-selective XMCD technique.

In addition to synchrotron radiation techniques, the anisotropy may be studied by magnetometry performed in conventional laboratories. One of the rarely used, but effective methods to directly study the magnetic anisotropy of a material is the transverse susceptibility (TS) technique. This measurement system consists in probing the ac magnetic susceptibility of a material in one direction while an external bias magnetic field is applied perpendicular to the measurement direction. Analysis of the TS in magnetic materials have demonstrated its potential and versatility to study singular magnetic properties of bulk $\lfloor 36-38\rfloor$, single crystals $\lfloor 39\rfloor$, thin films $\lfloor 40-43\rfloor$ and nanoparticles (NPs) systems [44-48]. TS measurement techniques developed up to date are based on conventional inductance susceptibility techniques $\lfloor 46,49,50\rfloor$, magnetooptical setups $\lfloor 40,43\rfloor$ and self-resonant circuits in the radio frequency (rf) range [51]. The latter method has demonstrated to be one of the most accurate and versatile developments to measure TS, since it may be implemented on widely used commercial Quantum Design physical properties measurement systems (PPMS). It consists of a self-resonant $L C$ tank coupled to a tunnel 
diode oscillator (TDO), the latter acting as an active device for losses compensation in the circuit. The magnetic sample is inserted into the inductor $(L)$ core space and its TS is probed by the shift in the resonant rf generated by changes in the sample magnetization.

However, currently operating TS measurement systems for the PPMS are far from being simple and friendly to use. Changing sample usually involves soldering and desoldering the inductance coil, jeopardizing the reproducibility of the system. Moreover, previously published designs of TS susceptometers for PPMS systems [51] introduce a high heat input into the sample space, avoiding the PPMS platform to stabilize the sample temperature below $10 \mathrm{~K}$, and, therefore, impeding the possibility of studying interesting lowtemperature phenomena with this technique. On the electronics design, it has been conventionally adopted the use of TDOs, which benefits of a good spectral purity, but limiting the compensation capabilities of the system and hence the range of samples feasible to measure. And finally, the necessity to install extra electronic and computing equipment to acquire data, independent to the control of the PPMS, makes the programming of experiments harder, rendering the previous systems not very efficient.

Then, in the second part of this thesis, we present an implementation of a TS measurement system for the PPMS of the Servicio de Medidas Físicas in the Universidad de Zaragoza. We have improved several aspects of the design, electronics, and data acquisition of previously published transversal susceptometers [51], turning this magnetometric technique a relatively easy one to implement and operate within the widely used PPMS platform. We also describe the use of the developed setup to explore the magnetic anisotropy of two systems of iron oxide nanoparticles, interesting for biomedical applications.

\section{Motivation and outline}

This dissertation is aimed to undertake an elaborate study of different magnetic nanoparticles systems, with which we intend to demonstrate the capabilities of two experimental techniques: the X-ray absorption spectroscopies (XANES, EXAFS and XMCD) and the rf transverse susceptibility. By using these techniques for electronic, structural and magnetic characterization of nanoparticles systems, along with other complementary methods, we obtain a better understanding of the origin of their magnetic anisotropy.

The manuscript is organized as follows. In Chapter $\mathbf{1}$ we describe the main experimental techniques used in the present work to study the structural and magnetic properties of NP systems. In particular, we focus on the 
X-ray absorption spectroscopies that make use of synchrotron radiation. In the following three chapters we describe the structural, magnetic and electronic properties of two nanoparticles systems, namely: Co-W NPs and Co-Pt NPs, studied with synchrotron radiation techniques. Hence, in Chapter 2 we describe the main properties of the amorphous Co-W alloy NPs. We demonstrate that the magnetic anisotropy of amorphous alloy nanoparticles may be originated in mechanisms other than surface effects, such as structural short range order. A continuation of this study is presented in Chapter 3, where we describe evidences of violation of Hund's third rule in the W induced magnetic moments of this $\mathrm{Co}-\mathrm{W}$ system, as consequence of hybridization effects and local structure in the Co-W alloys. Similarly, in Chapter 4 we describe the properties of the Co-Pt NPs studied, where the presence of Co-Pt alloy in these granular films is determinant in the origin of their perpendicular magnetic anisotropy. Then, a review of noble metals (Ag, Au, Pd, Pt) nanoparticles studied with synchrotron radiation is presented in Chapter $\mathbf{5}$, where we demonstrate that these techniques are very suitable to study their $d$-band magnetism.

The last two chapters of this thesis are dedicated to the rf transverse susceptibility (TS) technique. Chapter 6 is devoted to the description of the TS technique and the developed equipment for the PPMS. Then in Chapter 7 we present a couple of examples of magnetic NPs studied with this TS measurement system, namely magnetite and maghemite NPs. Finally, we give some general conclusions and perspectives of this work at the end of this manuscript. 



\section{Chapter 1}

\section{Experimental techniques}

This section is addressed to briefly describe the main experimental techniques used to study the structural and magnetic properties of the nanoparticles included in this thesis. We first explain the transmission electron microscopy techniques used to explore the morphology and structure of the particles. Then, we introduce two techniques to study the magnetic properties of the samples: SQUID based magnetometry and anomalous hall effect (AHE). Finally, we focus on the description of the synchrotron radiation techniques employed, in particular those based on X-ray absorption mechanisms, which allow studying selectively and locally the structural, electronic and magnetic properties of the nanoparticles systems; these are: X-ray absorption near edge structure (XANES), extended X-ray absorption fine structure (EXAFS), and $\mathrm{X}$-ray magnetic circular dichroism (XMCD).

Later, we will dedicate chapter 6 to describe the magnetic transverse susceptibility technique, which has been also used to study the magnetic properties of some nanoparticles systems in this dissertation.

\subsection{Transmission Electron microscopy}

The transmission electron microscopy (TEM) is a technique whereby an electron beam is transmitted through an ultra thin specimen, forming an image from the interaction between the transmitted electrons and the sample. TEMs are capable of imaging at a significantly higher resolution than light microscopes, owing to the small wavelength of electrons. This enables to examine fine details, even as small as a single column of atoms. This technique usually combines the high resolution imaging with elemental microanalysis and electron diffraction, so that a complete characterization of the shape, size, 
chemical composition and crystalline structure of nanoparticulate materials is achieved.

In a TEM microscope, an electron gun, located at the top of it, emits the electrons by thermionic or field emission. These electrons travel through vacuum in the column of the microscope where electromagnetic lenses focus them into a very thin beam. The electron beam then travels through the specimen to study. At the bottom of the microscope, the image is formed on the image plane of objective lens from the unscattered electrons. Then, projector lenses form the images on a screen or CCD camera.

One of the main requirements for this technique is that the sample must be thin enough for electrons to pass through; the interaction between electrons and matter is much stronger than that with visible light, so samples for TEM observation must have thickness below $100 \mathrm{~nm}$. There exists a variety of procedures to prepare samples for TEM observation, depending on the kind of materials and their nature. For example, bulk samples must be first cut to an proper size ( $\sim 3 \mathrm{~mm}$ in diameter, $300-400 \mu \mathrm{m}$ thick); after that, they are thinned, first mechanically and then by ionic or electrolytic milling, until reaching the desired thickness.

Some modern TEMs integrate a high angle annular dark field (HAADF) detector that allows to perform scanning transmission electron microscopy (STEM) measurements. In this mode, the electron beam is focused into a narrow spot that is scanned over the sample in a raster. The rastering of the beam across the sample makes these microscopes suitable for analysis techniques such as mapping by X-ray energy disperse spectrometry (EDX) spectroscopy and electron energy loss spectroscopy (EELS). The HAADF detector collects electrons from an annulus around the beam, forming the image only by very high angle incoherently scattered electrons, as opposed to Bragg scattered electrons in the conventional TEM images. By using a STEM and the HAADF detector, it is possible to form atomic resolution images where the contrast is directly related to the atomic number (Z-contrast image).

The microscopy images and analysis of the magnetic nanoparticles in this work were performed with two TEMs, whose technical specifications are described below.

\subsubsection{JEOL JEM $3000 \mathrm{~F}$}

A JEOL JEM 3000 F Field Emission Microscope has been used by Dr. Luisa Ruiz González and Dr. José María González-Calbet of the Departamento 


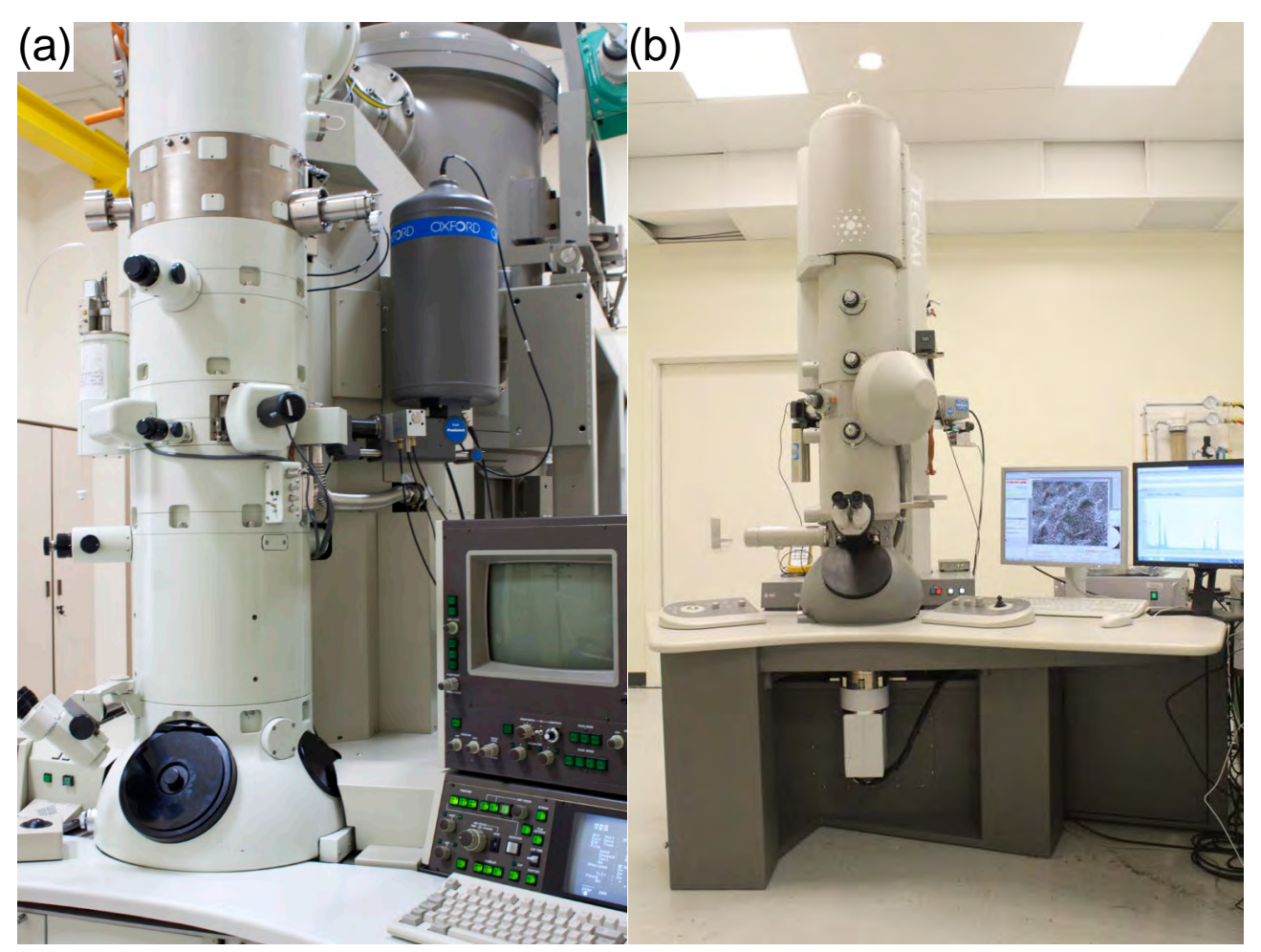

Figure 1.1: Images of the two models of transmission electron microscopes used to observe the samples. (a) JEOL JEM 3000 F Field Emission Microscope. (b) FEI Tecnai ${ }^{\mathrm{TM}}$ G2 F30 STWIN Field Emission Microscope.

de Química Inorgánica in the Universidad Complutense de Madrid ${ }^{1}$ to take some of the images of the nanoparticles systems that we show in this thesis. This microscope is shown in Fig. 1.1(a). It operates at acceleration voltages between 100 and $300 \mathrm{kV}$ and it is fitted with an Oxford Instruments energy dispersive X-ray spectrometer (EDS). Its high-resolution performance (a point resolution of $0.17 \mathrm{~nm}$ ) is then complemented by the ability to perform chemical microanalysis from sub-nanometre areas (below $0.4 \mathrm{~nm}$ in some cases). This microscope is equipped with a HAADF detector, so that STEM mode and EELS analysis are also possible.

\subsubsection{FEI Tecnai ${ }^{\mathrm{TM}}$ G2 F30 STWIN}

Some of the TEM images that we show in this dissertation have taken by Dr. Cesar Magén and Dr. Alfonso Ibarra in a FEI Tecnai ${ }^{\text {TM }}$ G2 F30 STWIN Field Emission Microscope of the Laboratorio de Microscopías Avanzadas,

\footnotetext{
${ }^{1}$ http://www. cnme.es/
} 
Instituto de Nanociencia de Aragón, ${ }^{2}$ Universidad de Zaragoza. A picture of a microscope of this kind is shown in Fig. 1.1(b). In this microscope, acceleration voltages as high as $300 \mathrm{kV}$ allow reaching a point resolution of 0.2 $\mathrm{nm}$, line resolution of $0.10 \mathrm{~nm}$, and information limit of $0.14 \mathrm{~nm}$. This TEM system is fully loaded including a HAADF detector, EDX, and a Gatan Image Filter (GIF).

\subsection{SQUID based magnetometry}

A magnetometer is an instrument used for the magnetic characterization of a material, by measuring the strength and/or direction of magnetic fields. A superconducting quantum interference device (SQUID) is the most sensitive device available for such purposes. It works as an extremely sensitive currentto-voltage convertor, so that, when integrated to a Quantum Design magnetic property measurement system (MPMS), the complete system is a magnetic flux to voltage transducer. The fact of directly measuring magnetic flux is one of the main advantages of SQUID magnetometry, allowing to measure moments on the order of $10^{-7} \mathrm{emu}$.

A picture of one of the MPMS of the Servicio de Medidas Físicas of the Universidad de Zaragoza, ${ }^{3}$ which have been used for some of the magnetic characterization described in this manuscript, is shown in Fig. 1.2(a). A schematics of the measurement process of a magnetic sample in this equipment is displayed in Fig. 1.2(b). In a MPMS, the SQUID does not detect directly the magnetic field from the sample since it may be interfered by any flux from nearby magnetic systems, or even the earth's magnetic field. Instead, a measurement is performed by moving a sample through a system of superconducting pick-up coils, two of them with the same sense very closely spaced, and the other two of the opposite sense, spaced symmetrically outside (see Fig. 1.2(b)). This system is located outside the sample chamber at the center of the superconducting magnet. As the sample moves through the coils, the magnetic moment of the sample induces an electric current in the detection coils. Because the detection coils, the connecting wires, and the SQUID input coil form a closed superconducting loop, any change of magnetic flux in the detection coils produces a change in the persistent current in the detection circuit, which is proportional to the change in magnetic flux. Since the SQUID is a highly linear current-to-voltage convertor, the variation in the current in the detection coils produces corresponding variations in the SQUID output voltage. Based on this profile of voltage (and thus flux) versus position

\footnotetext{
${ }^{2}$ http://ina.unizar.es/lma/index.html

${ }^{3}$ http://sai.unizar.es/medidas/index.html
} 

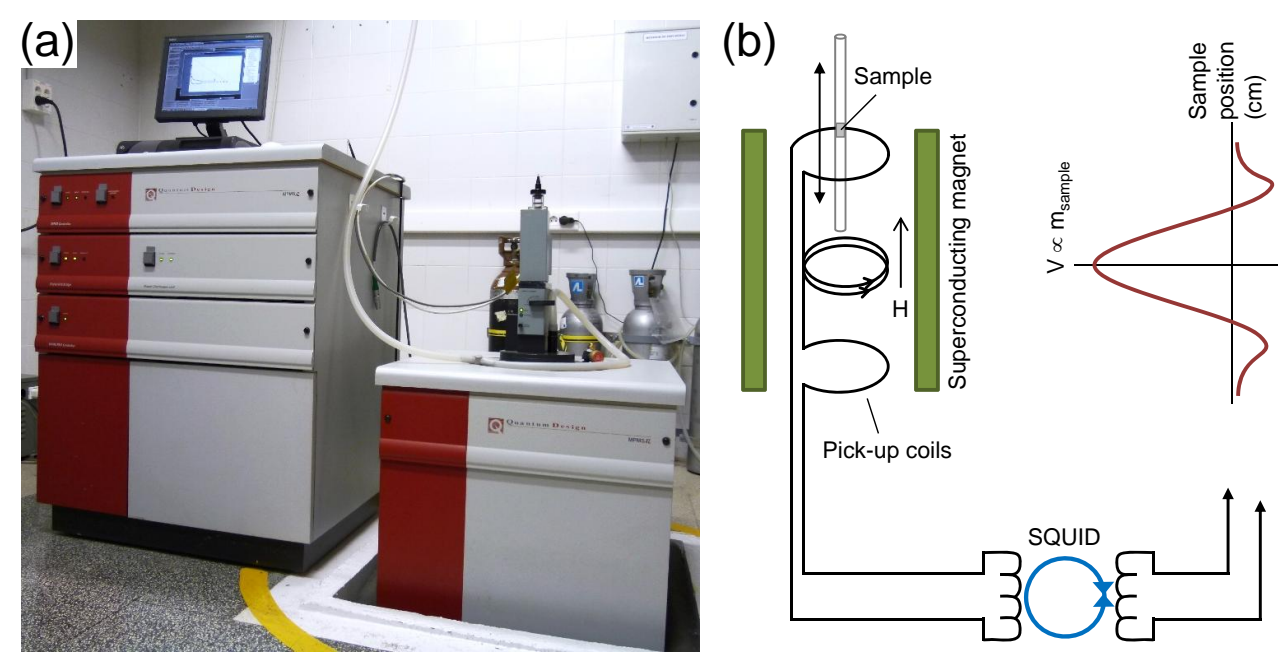

Figure 1.2: (a) MPMS-XL magnetometer of the Universidad de Zaragoza. (b) Schematics of the SQUID - based measurement system.

of the sample (see Fig. 1.2(b)), given the assumption that the signal is from a point dipole, the magnetic moment can be determined.

This MPMS allows measuring magnetization as a function of magnetic fields up to $50 \mathrm{kOe}$, or temperature in a range of 1.9 - $400 \mathrm{~K}$. AC measurements with a field amplitude of 4 Oe and frequencies from 0.01 to $1000 \mathrm{~Hz}$ are also possible in this equipment. It integrates a reciprocating sample option (RSO), which consists of a high resolution mode to improve the equipment sensitivity to measure moments as small as $5 \times 10^{-9}$ emu. Unlike DC measurements where the sample is moved through the coils in discrete steps, the RSO measurements are performed using a servo motor which rapidly oscillates the sample. A digital signal processor (DSP) is incorporated in the RSO, which allows for much more rapid data collection than the standard step scan method. The DSP also decreases the contribution of low frequency noise during data collection, which improves the signal-to-noise ratio resulting in maximum sensitivity. ${ }^{4}$

\subsection{Anomalous Hall Effect}

The Hall effect [53. (or ordinary Hall effect, OHE) is a well known and studied phenomenon, characterized by the production of a voltage difference (the Hall voltage) across an electrical conductor or semiconductor, transverse to an elec-

\footnotetext{
${ }^{4}$ For further information of SQUID magnetometers see Ref. 52. and/or the Quantum Design website http://www.qdusa.com/index.html
} 
tric current in the material, and a magnetic field perpendicular to the current. In magnetic materials, there is an additional contribution to the Hall voltage, proportional to the magnetization, called the extraordinary or anomalous Hall effect (AHE). Correlation between the AHE and magnetization enables the use of the Hall voltage measurement for the study of magnetic properties of materials in conditions hardly accessible by other techniques [54].

The Hall voltage in magnetic materials is commonly described by the phenomenological equation $[55,56\rfloor$

$$
V_{H}=\underbrace{\frac{R_{H} I}{t} H \cos (\alpha)}_{O H E}+\underbrace{\frac{\mu_{0} R_{s} I}{t} M \cos (\theta)}_{A H E}+\underbrace{\frac{k I}{t} M^{2} \sin ^{2}(\theta) \sin (2 \phi)}_{P H E}
$$

where $t$ is the film thickness, $R_{H}, R_{s}$ and $k$ are the ordinary, anomalous and planar Hall coefficients, respectively, and the angles $\alpha, \theta$ and $\phi$ are defined in Fig. 1.3. The first term in equation 1.1 is the OHE and arises from the Lorentz force acting on conduction electrons. The OHE depends on the $z$-component of the $\vec{H}$ field, and produces an electric field perpendicular to $H_{z}$ and the current $I$. The second term is the AHE and arises due to spin dependent scattering mechanisms. It is is associated with a break of the right-left symmetry during the spin-orbit scattering in magnetic materials and its contribution could be much larger than the OHE. The AHE depends on the perpendicular component of $\vec{M}$, and produces an electric field perpendicular to $M_{z}$ and $I$. The last term in equation 1.1 is the planar Hall

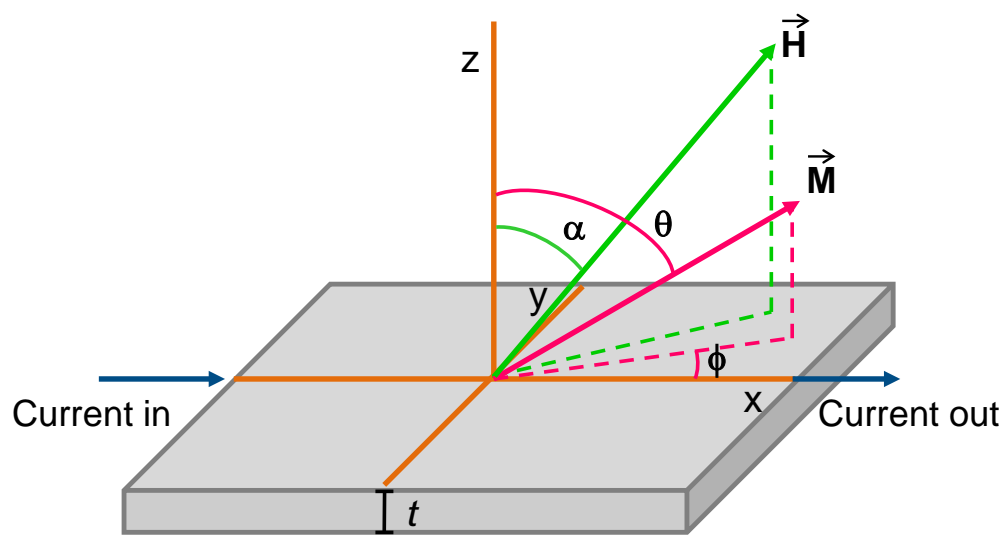

Figure 1.3: Geomery of the AHE measurement. $\alpha$ is the angle between the applied field and the normal to the sample, $\theta$ is the angle between the magnetization and the normal and $\phi$ is the angle between the current and the in plane component of the magnetization. 
effect (PHE), or anisotropic magneto-resistance. The PHE is proportional to the square of the planar component of $\vec{M}$, and produces an electric field parallel and perpendicular to the current (the one expressed in equation 1.1 is perpendicular to the current). Note that all three terms are inversely proportional to the film thickness $t$. Given this property, the AHE has been recognized as a useful tool for measuring the magnetic hysteresis $\mathrm{M}(\mathrm{H})$ loops of thin films, specially those used in perpendicular magnetic recording media (PMRM), ferromagnetic/semiconductor heterostructures (spintronic devices), and diluted-magnetic-semiconductors $[55,56\rfloor$.

The AHE measurements performed on some of the nanoparticles systems described in this thesis were carried out by Dr. Jolanta Stankiewicz in an electrical transport setup of the Departamento de física de la materia condensada and Instituto de Ciencia de Materiales de Aragón (ICMA), CSIC - Universidad de Zaragoza, and in a Quantum Design Physical Property Measurement System (PPMS) of the Servicio de Medidas Físicas of the Universidad de Zaragoza. $^{5}$

\subsection{X-ray absorption spectroscopies in synchrotron radiation facilities}

Several experimental techniques based on interactions of radiation with matter require of highly intense, bright, continuous and tuneable light sources $\lfloor 57,58\rfloor$. These requirements are easily fulfilled with synchrotron radiation sources. These large-scale facilities consist of particle accelerators that speed up charged particles, such as electrons or protons, into an orbit at almost the speed of light. According to the electromagnetic theory, an electric charge irradiates energy when accelerated. This radiation is emitted tangentially to the circular trajectory of the electric charge when it is deflected. Third generation synchrotron radiation facilities are the most powerful currently operating synchrotron light sources, and have become one of the essential tools for basic and applied scientific research over the world.

The main properties of the synchrotron light may be summarized as follows: it has a broad spectrum from microwaves to hard X-rays, so that it is a tuneable radiation source; its high intensity photon beam of about $10^{18}-10^{20}$ photons $/ \mathrm{s} / \mathrm{mm}^{2}$ (compared to the $10^{8}$ photons $/ \mathrm{s} / \mathrm{mm}^{2}$ of a conventional X-ray tube) allows rapid experiments; it is a highly brilliant photon beam generated by a small divergence and small size source (spatial coherence); it is a very stable (submicron source stability) and polarized radiation.

\footnotetext{
${ }^{5} \mathrm{http}: / /$ sai.unizar.es/medidas/index.html
} 
A diagram of a synchrotron radiation facility is shown in Fig. 1.4. The synchrotron light generation starts in an electron (or proton) gun (1), where a cathode produces free electrons released to a linear accelerator or LINAC (2). The LINAC feeds a booster ring (3), where electrons receive a boost in energy from approximately $250 \mathrm{MeV}$ to approximately 1.5 to $8 \mathrm{GeV}$ from microwaves generated in a radio frequency cavity as they circulate in the ring. The booster increases the speed of the electrons close to the speed of light. When the electrons have enough energy to produce light, an injection system transfers them from the booster ring to the storage ring (4): a tube with straight and circular sections kept in ultra high vacuum, with quadrupole and sextupole magnets designed to focus the electron beam. Once there, the electrons will circulate for a long period of time producing photons every time the dipole magnets (also called bending magnets) change the direction of the flow of electrons. After each turn there is a photon port to allow the light to travel down the beamlines to the research stations. Aside from the bending magnets, synchrotron light may also be produced by insertion devices, such as wigglers or undulators, located at the straight sections of the storage ring. They consist of periodic magnetic structures that stimulate highly brilliant, forward-directed synchrotron radiation emission by forcing the stored charged particle beam to perform wiggles, or undulations, as they

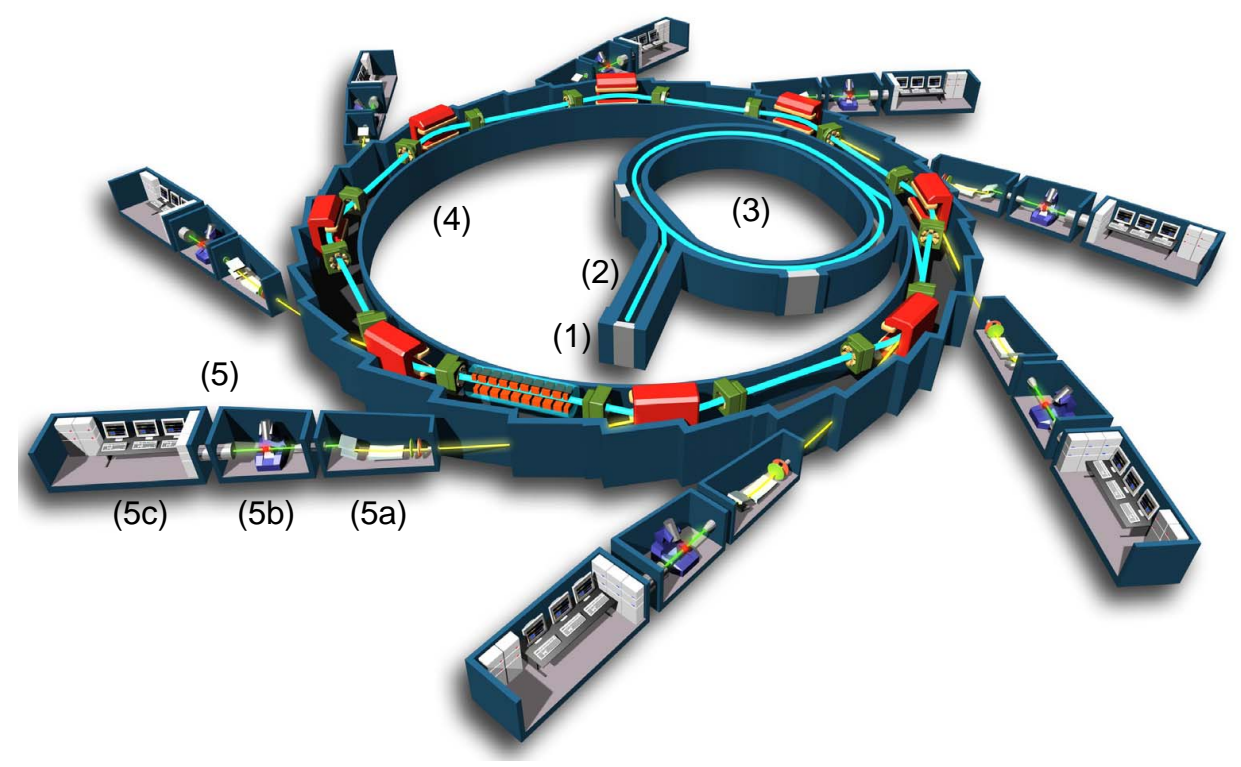

Figure 1.4: General diagram of a synchrotron facility. ${ }^{6}(1)$ Electron or proton gun, (2) LINAC, (3) booster, (4) storage ring, (5) beamline, (5a) optics cabin, (5b) experimental hutch, and (5c) control cabin.

\footnotetext{
${ }^{6}$ General diagram of Synchrotron Soleil with Copyright CEPSIM 3D/JF Santarelli.
} 
pass through the device. The magnet arrays are designed in such a way that the emitted radiation interferes constructively to produce a very intense and concentrated beam in narrow energy bands in the spectrum.

Each beamline has an optics cabin (5a), an experimental hutch (5b), and a control cabin (5c). The optics cabin has optical instruments used to tailor the type of radiation to have the characteristics for each experiment. The experimental hutch contains the support mechanism, and the environment for the sample study. Different instruments and detectors record the information produced from the interaction of the synchrotron light and the sample. The control cabin allows the researchers to control the experiments and collect the data.

The synchrotron radiation source used to study the magnetic nanoparticles in this dissertation is the European Synchrotron Radiation Facility (ESRF), ${ }^{7}$ which is a third generation and the most powerful synchrotron in Europe. It is located in Grenoble, France. It operates at a nominal energy of $6 \mathrm{GeV}$, with a circumference of $844 \mathrm{~m}$, and around 49 operating beamlines.

$\mathrm{X}$-ray absorption spectroscopy (XAS) is one of those techniques that is best performed with the intense and tunable X-ray beams produced in a synchrotron radiation source. This spectroscopy refers to the details of how $\mathrm{X}$-rays are absorbed by an atom at energies near and above the core-level binding energies of that atom. The absorbing process is illustrated in figure 1.5(a). An X-ray photon reaches the absorbing atom (in blue) and disappears. After the interaction, a photoelectron is emitted. Depending on the energy of the incident $\mathrm{X}$-ray photon, different processes occur. If the binding energy of certain electron in the absorbing atom is equal to the $\mathrm{x}$-ray photon energy, there is a sharp increase in the absorption cross-section, called absorbing edge, corresponding to an emission of the photoelectron to the continuum states, as indicated in $1.5(\mathrm{~b})$. The absorption edges are named according to the principle quantum number of the electron that is excited: $K$ for $n=1, L$ for $n=2, M$ for $n=3$, and so on. The energies at which edges appear are unique for each element, fact that makes this spectroscopy an element-selective technique.

\subsubsection{X-ray absorption fine structure}

$\mathrm{X}$-ray absorption fine structure (XAFS) is a specific structure observed in XAS $[59,60]$. A usual XAFS spectrum can be seen in Fig. 1.6, where the two spectral regions are identified: the near-edge region XANES, typically within $50 \mathrm{eV}$ of the main absorption edge, and the extended fine-structure (EXAFS) which spans the 50 to $2000 \mathrm{eV}$ above the edge. The basic physical description

\footnotetext{
${ }^{7}$ http://www.esrf.eu/
} 
(a)

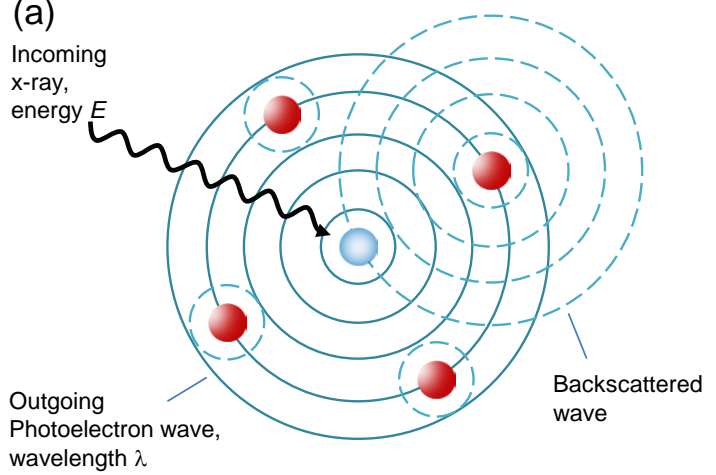

(b)

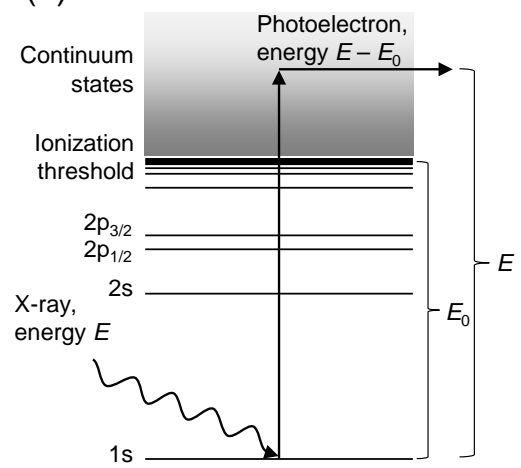

Figure 1.5: Schematics of the X-ray absorption process. (a) An absorbing atom (blue) is shown here surrounded by several other atoms (red). Whenever the energy of an $\mathrm{X}$-ray is greater than the binding energy of one of the absorbing atom's core electrons, a photoelectron is liberated. (b) Energy-level diagram of an absorbing atom showing the atom's first few core-electron orbitals: $1 s, 2 s, 2 p_{1 / 2}$, and $2 p_{3 / 2}$. The ionization potential energy is denoted by $E_{0}$. With the absorption of a photon of energy $E$, the electron undergoes a transition to an unbound state in the continuum and, by conservation of energy, acquires a kinetic energy $\left(E-E_{0}\right)\lfloor 59\rfloor$.

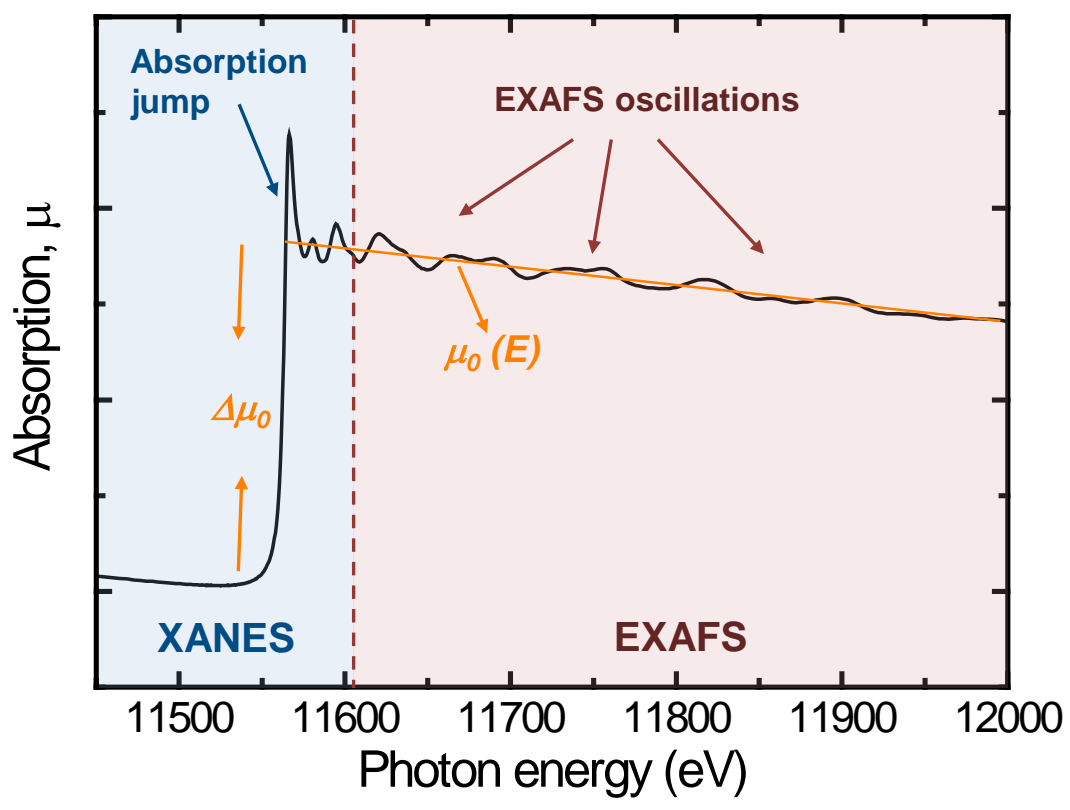

Figure 1.6: XAFS spectrum recorded at the $\mathrm{Pt} L_{3}$ edge for a $\mathrm{Pt}$ foil at $300 \mathrm{~K}$. We have divided the spectrum into its two main regions: XANES - X-ray Absorption Near Edge Structure, and EXAFS - Extended X-ray Absorption Fine Structure. The background function $\mu_{0}$, and the measured jump at the absorption at the threshold energy $E_{0}, \Delta \mu_{0}$, are also shown. 
of these two regimes is the same. EXAFS results from the interference in the single scattering process of the photoelectron scattered by surrounding atoms (see Fig. 1.5(a)), and it provides information on the local structure such as distances between neighbors, thermal and structural disorder in a material. Information on the geometry of the local structure is provided by the analysis of the multiple scattering peaks in the XANES spectra. However, XANES is harder to be described in a quantitative way since there is not an equation to parameterize this region, while it does exist for EXAFS, as it will be described below.

The X-ray absorption process is a transition between two quantum states: from an initial state with an X-ray, a core-electron, and no photoelectron, to a final state with no X-ray, a core-hole, and a photoelectron. According to the Fermi's golden rule for dipolar transitions (following the dipole selection rules $\Delta l= \pm 1$ and $\Delta s=0$ ), the transition probability between two electronic states, per unit time, is defined as

$$
W_{i f}=\frac{2 \pi}{\hbar}\left(\frac{e E_{0}}{2}\right)^{2}|\langle i|\hat{\varepsilon} \cdot \vec{r}| f\rangle|^{2} \rho\left(E_{f}\right)
$$

where $\rho\left(E_{f}\right)$ is the density of final states, $\langle i|$ is the initial state of the coreelectron, $|f\rangle$ the final state of the photoelectron, and $\hat{\varepsilon} \cdot \vec{r}$ the dipole operator. Since the core-level electron is very tightly bound to the absorbing atom, the initial state will not be altered by the presence of the neighboring atom. The final state, on the other hand, will be affected by the neighboring atom because the photoelectron will be able to see it. The absorption coefficient $\mu(E)$ is proportional to the sum of possible final states $f$ of the transition probability in equation 1.2. It is calculated according to the first order approximation of the time-dependent perturbation theory, based on the interaction between the electromagnetic field and the atom potential.

In the EXAFS region, the spectrum reflects oscillatory variation of the $\mathrm{X}$-ray absorption coefficient, $\mu(E)$, as a function of photon energy, $E$, beyond the absorption edge (see Fig. 1.6). We define the EXAFS fine-structure function $\chi(E)$ as

$$
\chi(E)=\frac{\mu(E)-\mu_{0}(E)}{\Delta \mu_{0}(E)}
$$

where $\mu(E)$ is the measured absorption coefficient, $\mu_{0}(E)$ is a smooth background function representing the absorption of an isolated atom, and $\Delta \mu_{0}(E)$ is the measured jump in the absorption $\mu(E)$ at the threshold energy $E_{0}$. These parameters are also represented in Fig. 1.6. 
According to the described absorption process, the EXAFS $\chi(E)$ is proportional to the amplitude of the scattered photoelectron at the absorbing atom. Therefore, EXAFS is best understood in terms of the wave-like nature of the photoelectron created in this process. Thus, it is common to convert the $\mathrm{X}$-ray energy to $k$, the wave number of the photoelectron, being $k^{2}=2 m\left(E-E_{0}\right) / \hbar^{2}$, and $m$ the electron mass. The primary quantity for EXAFS is then $\chi(k)$, the oscillations as a function of photoelectron wave number. To emphasize the oscillations, $\chi(k)$ is often weighted by a power of $k$, typically $k^{2}$ or $k^{3}$. The different frequencies composing these oscillations correspond to different near-neighbor coordination shells, described and modeled according to the EXAFS equation

$$
\chi(k)=\sum_{j} \frac{N_{j} S_{0}^{2} F_{j}(k) \exp \left(-2 k^{2} \sigma_{j}^{2}\right) \exp \left(-\frac{2 R_{j}^{2}}{\lambda_{j}(k)}\right)}{k R_{j}^{2}} \sin \left(2 k R_{j}+\delta_{j}(k)\right)
$$

where $F_{j}(k)$ is the backscattering amplitude from each of the $N_{j}$ neighboring atoms, $\delta_{j}(k)$ is the total phase shift experienced by the photoelectron, $R_{j}$ is the distance to the neighboring atom $j, \sigma_{j}^{2}$ is the disorder in the neighbor distance, parameter usually known as Debye-Waller factor, and $S_{0}^{2}$ is the amplitude reduction due to many-body effects. The term $\exp \left(-2 R_{j}^{2} / \lambda_{j}(k)\right)$ is due to inelastic losses in the scattering process, with $\lambda_{j}$ being the electron mean free path. The EXAFS equation allows us to determine $N, R$, and $\sigma^{2}$ knowing the scattering amplitude $F(k)$ and phase-shift $\delta(k)$ for each path. Furthermore, since these scattering factors depend on the atomic number, Z, of the neighboring atom, EXAFS is also sensitive to the atomic species of the neighborhood.

The EXAFS $\chi(k)$ is usually Fourier transformed into a radial structure function $\chi(R)$ that represents the data in terms of distances from the absorber. Peak positions in this radial structure function roughly correspond to the radii of the scattering shells.

\section{BM29 beamline at the ESRF}

The XAFS experiments on the magnetic nanoparticles system studied here were performed in collaboration with Dr. Sakura Pascarelli at the general purpose X-ray absorption spectroscopy beamline BM29 [61」 at the ESRF. ${ }^{8}$

\footnotetext{
${ }^{8}$ In a recent upgrade, performed in 2011, the ESRF BM29 beamline has been relocated to the BM23 port, which is the one currently operational. For further information see http://www.esrf.eu/UsersAndScience/Experiments/ElectStructMagn/BM23/
} 
This beamline is devoted to carry out XAFS experiments at a very large operational energy range with reasonable X-ray flux: $4 \mathrm{keV}$ to $74 \mathrm{keV}$, coming from a bending magnet. It has a high energy resolution, typically a factor 3 to 5 better than the intrinsic spectral broadening at any $K$ or $L$ absorption edge; a high spectral signal to noise ratio, above $7.0 \times 10^{4}$ for well prepared samples; a high beam stability; and a high level of automation.

A schematic diagram of a conventional XAFS beamline, as BM29, is shown in Fig. 1.7. The full spectrum of synchrotron light passes through an entrance slit, which helps collimate the radiation before entering a into a double-crystal monochromator. The $\mathrm{X}$-rays diffract off the crystals' lattice planes, which are aligned at an angle $\theta$ with respect to the incoming beam. Because of the Bragg scattering condition $n \lambda_{j}=2 d \sin \theta$, only X-rays of energy $E_{n}=n h c / \lambda_{j}$ can reflect off the crystals. Different $\mathrm{X}$-ray energies can be selected by changing the angle $\theta$ of the crystals. The monochromatic beam passes through an ion chamber that monitors the beam intensity $I_{0}$ by absorbing part of it. The beam then passes through the sample, and the intensity of the transmitted $\mathrm{X}$-ray $I$ is measured by a second ion chamber. Taking the log of the ratio of $I / I_{0}$ yields the absorption. We can also determine the sample's absorption by measuring the intensity of its $\mathrm{X}$-ray fluorescence, $I_{f}$ with a detector that is out of the beam path, usually oriented at $45^{\circ}$ with respect to the sample. Finally, the beam passes through a reference sample whose absorption edge is used to help correct for uncertainties in the beam's energy.

\subsubsection{X-ray magnetic circular dichroism}

Some synchrotron radiation techniques take advantage of the natural polarization of this light in the plane of the electron orbit in order to perform polarization-dependent measurements. This is the case of the X-ray magnetic circular dichroism (XMCD), where the difference between two X-ray absorption spectra recorded in a magnetic field, one measured with right circularly polarized light ( +1 helicity), and one with left circularly polarized light (-1 helicity), is performed. The XMCD is then defined as

$$
X M C D(E)=\mu^{-}-\mu^{+}
$$

where $\mu^{-}$and $\mu^{+}$are the absorption coefficients with left and right circularly polarized light, respectively. The differences in the absorption of light with opposite helicities arise from an additional dipole selection rule for dipolar transitions, $\Delta m_{j}= \pm 1$ for +1 and -1 helicity, respectively, in equation 1.2; the dipole operator, $\hat{\varepsilon} \cdot \vec{r}$, must also be expressed as dependent of the light polarization. This way, XMCD reflects the difference in the density of 


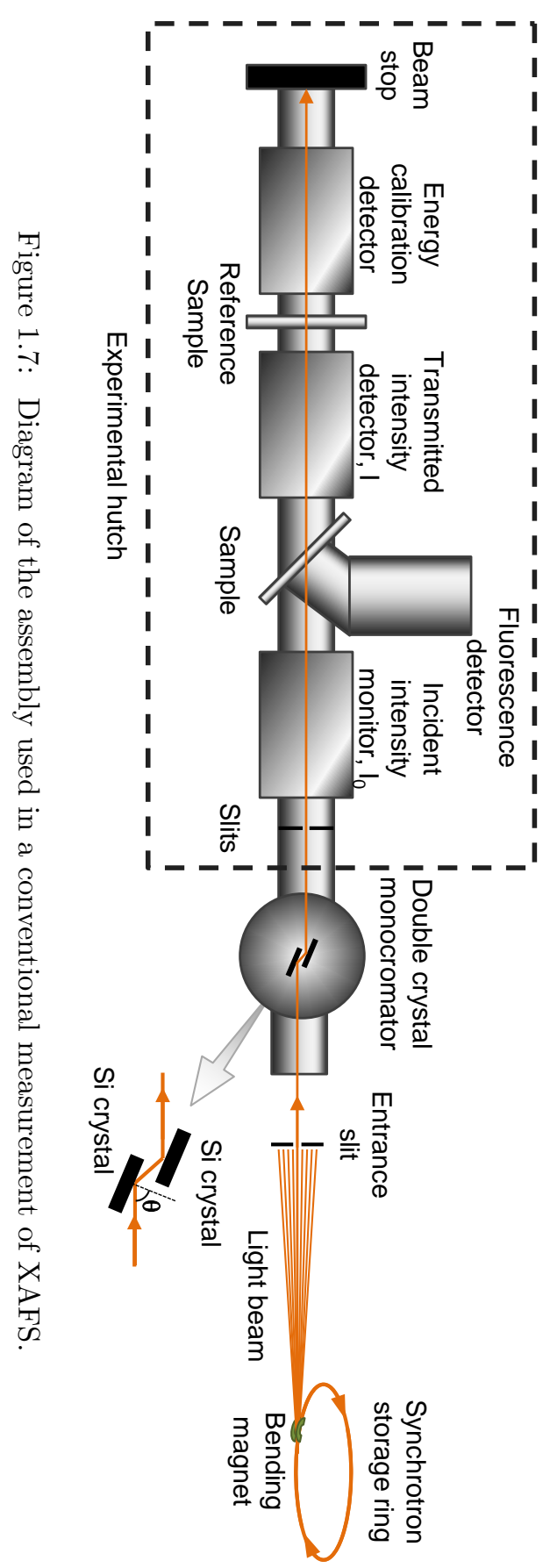


empty states with different spin moment, so that by closely analyzing the XMCD signal, information can be obtained on the magnetic properties of the absorbing atom, such as its spin and orbital magnetic moment.

In a simple atomic monoelectronic picture, the XMCD occurrence may be understood in terms of the two-step approach described by Schütz et al. [62]. Let us first consider an X-ray photon with helicity +1 absorbed by an electron from a spin-orbit-split core-level, for example, $2 p_{3 / 2}\left(L_{3}\right.$ edge) and $2 p_{1 / 2}\left(L_{2}\right.$ edge), as illustrated in Fig. 1.8(a). When absorbing a circularly polarized photon, some of the orbital moment of the photon can be transferred via the spin-orbit coupling to the electron spin. This results in an effective spin polarization of the exited electrons, process known as Fano-effect [63]. From
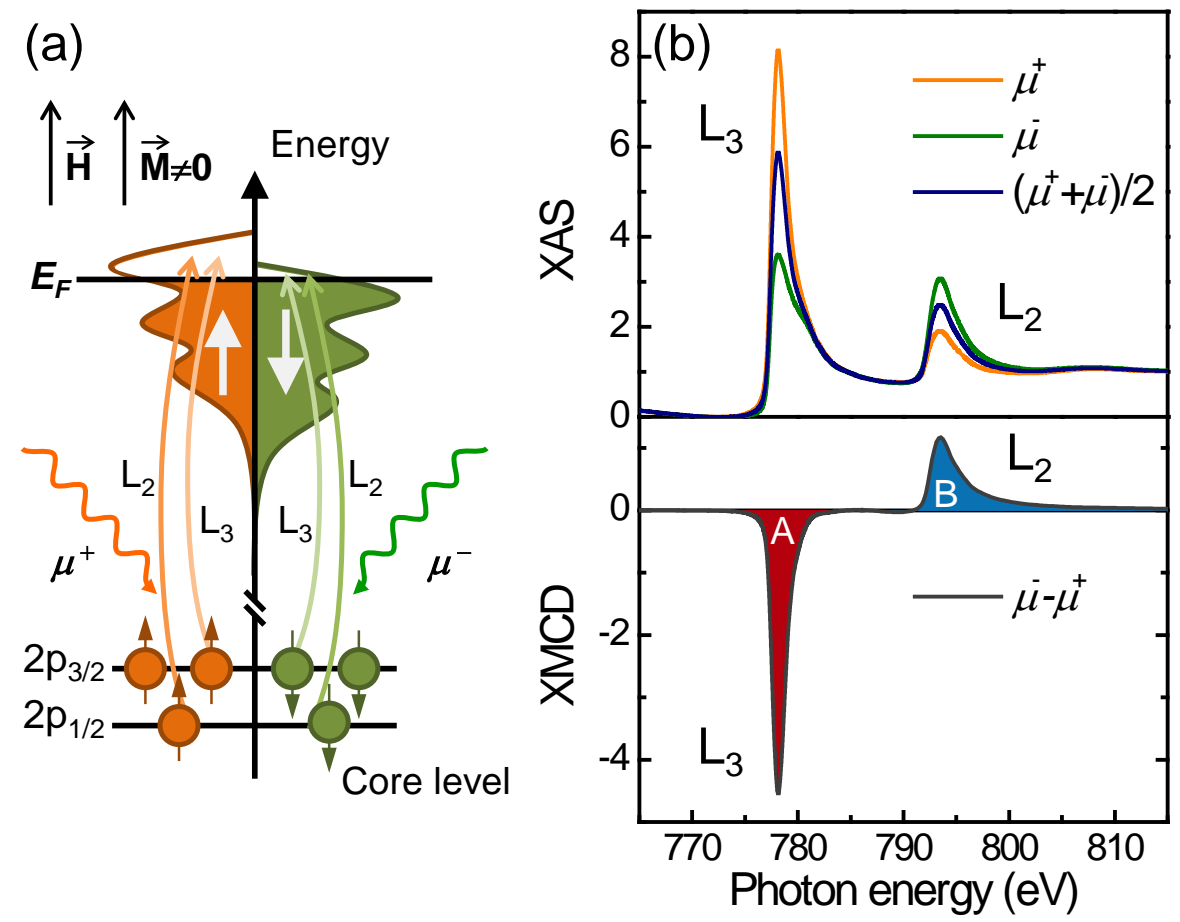

Figure 1.8: (a) Diagram of the simple monoelectronic two-step picture of the resonant excitation process in a magnetic material, resulting in a XMCD signal. First a circularly polarized photon excites a spin polarized electron (Fano-effect) from a $2 p$ level; this spin polarized electron is used in step two to probe the spin polarized occupation levels of the $3 d$ states. (b) XAS spectra and XMCD signal at the Co $L_{2,3}$ in a system of Co-Pt NPs. In this case, a scan over the $2 p$ absorption edges of Co with circularly polarized light leads to non statistic absorption lines due to differently spin occupied $3 d$ states. This effect is reversed by inverting either the magnetization (change of the magnetic field direction) or the light polarization. In this picture the polarization of the light is reversed between the orange and green absorption curves. The difference of left and right circularly polarized scan is the dichroic signal displayed below. 
the $2 p_{3 / 2}$ state, $62.5 \%$ of excited electrons carry a spin of $m_{s}=+1 / 2$ and only $37.5 \%$ of $m_{s}=-1 / 2$. From the $2 p_{1 / 2}$ state, $75 \%$ of excited electrons carry a spin of $m_{s}=-1 / 2$ and only $25 \%$ of $m_{s}=+1 / 2$. Note that the sign of spin polarization is different for the different $2 p$ states, provided their different spin-orbit coupling: $(l+s)$ at the $p_{3 / 2}$-state, and $(l-s)$ at the $p_{1 / 2}$-state. If there were no spin-orbit-splitting, that is, no energy gap between $2 p_{3 / 2}$ and $2 p_{1 / 2}$ states, the averaged spin polarization would be zero.

With this spin and orbital polarized excited electrons, the polarization of the unoccupied final states is probed in the second step of this model. Any imbalance in either spin or orbital momentum in the final states will give rise to a dichroic effect. Thus, in a magnetic material, the exchange splitting leads to a splitting of the valence band, lowering the energy for electrons with one spin orientation (majority electrons) and rising it for the other (minority electrons), as illustrated in Fig. 1.8(a). The transition probability of electrons in a dipole transition are proportional to the unoccupied density of states, as expressed in equation 1.2. If this density of states is different for different spin orientations and the electrons are spin polarized, the absorption spectra will change when the spin polarization is inverted, as due to the change of the helicity of the incoming light. This results in spectra as the one represented in Fig. 1.8(b), where we have plotted the XAS recorded with right and left circularly polarized X-rays at the Co $L_{2,3}$ edges in a Co-Pt NPs sample, along with the resulting XMCD signal.

To see this effect it is required that the magnetization of the sample has at least a component parallel to the incoming light. The inversion of the magnetization has the same effect on the absorption process as the inversion of the photon polarization. Thus, XMCD measurements can be performed in those two equivalent ways: either by changing the helicity while keeping constant the applied magnetic field, or by changing the direction of the magnetic field at a constant helicity of the light.

XMCD became more powerful as a tool for the element-selective characterization of magnetic materials with the derivation of the magneto-optical

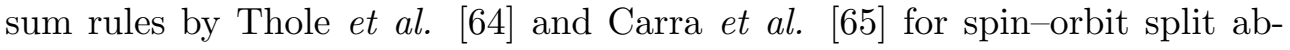
sorption edges. Via these sum rules it is possible to determine both the spin, $m_{\mathrm{S}}=-2 \mu_{B}\left\langle S_{z}\right\rangle / \hbar$, and orbital, $m_{\mathrm{L}}=-\mu_{B}\left\langle L_{z}\right\rangle / \hbar$, magnetic moments in the atom being probed with the XMCD measured at its $L_{2,3}$ edges. In order to write the sum rules, let us define the following integrals 


$$
\begin{aligned}
& A=\int_{L_{3}}\left(\mu^{-}-\mu^{+}\right) d E \\
& B=\int_{L_{2}}\left(\mu^{-}-\mu^{+}\right) d E \\
& C=\int_{L_{3}+L_{2}}\left(\mu^{-}+\mu^{+}+\mu^{0}\right) d E
\end{aligned}
$$

which follow the symbols and sign criteria denoted in Ref. [66]. The absorption spectrum $\mu^{0}$ requires in principle linearly polarized radiation, but is usually approximated by $\mu^{0}=1 / 2\left(\mu^{+}+\mu^{-}\right)$. Before application of the sum rules it is necessary to separate the absorption intensity due to the electron transitions we are interested in. Thus, the $C$ integral must be calculated after removing the unwanted transitions into higher unoccupied states or into the continuum in the absorption spectra. It is usually performed by subtraction of a hyperbolic step function with one step at the $L_{3}$ and one at the $L_{2}$ edge $[67\rfloor$. If there are less pronounced absorption maxima at the absorption edges, a reference spectrum of a similar element with a well-known number of unoccupied states should be subtracted.

The sum rule for the orbital moment per unoccupied final state $n_{h}$ is expressed as

$$
\frac{m_{\mathrm{L}}}{n_{h}}=-\frac{2(A+B)}{C} \mu_{B}
$$

For the spin sum rule, it is usual to define the effective spin moment, $m_{\text {Seff }}=m_{\mathrm{S}}+m_{\mathrm{D}}$, which considers the dipolar term $m_{\mathrm{D}}$. The latter is expressed as a function of the expectation value of the intra-atomic magnetic dipole operator $\left\langle T_{z}\right\rangle\lfloor 66,68\rfloor$, as $m_{\mathrm{D}}=-7\left\langle T_{z}\right\rangle \mu_{B} / \hbar$, which reflects the asphericity of the spin moment distribution around the absorbing atom. In order to determine $m_{\mathrm{S}}$, one option is to assume that the angle averaged $\left\langle T_{z}\right\rangle$ is much smaller than the spin moment and can be neglected, as in the case where the absorbing atom is in a cubic (undistorted) symmetry. On the contrary, for lower symmetries or distorted systems, $\left\langle T_{z}\right\rangle$ may be obtained from angle dependent experiments or theoretically approximated $[68,69\rfloor$. For example, for non-cubic structures, $\left\langle T_{z}\right\rangle$ might have a maximum value of $\left\langle S_{z}\right\rangle / 3$, corresponding to the Lorentz local field at the absorbing atom site $[70\rfloor$. However, the angle average of $\left\langle T_{z}\right\rangle$ with this approximation in the whole sample usually cancels out, as well. 
If the angle average of $\left\langle T_{z}\right\rangle$ is zero, $m_{\text {Seff }}=m_{\mathrm{S}}$, and the spin moment, also per $n_{h}$, is written as

$$
\frac{m_{\mathrm{S}}}{n_{h}}=-\frac{3(A-2 B)}{2 C} \mu_{B}
$$

Therefore, the orbital to spin moment ratio is defined as

$$
\frac{m_{\mathrm{L}}}{m_{\mathrm{S}}}=\frac{4(A+B)}{3(A-2 B)}
$$

which is independent of $n_{h}$ and the $C$ integral. For a precise estimation of these moments, one must separate the $L_{3}$ and the $L_{2}$ contribution of the spectrum, which is fairly easy for the heavier $3 d$, and for $4 d$ and $5 d$ elements since the spin-orbit split increases with the nuclear charge of the atom. Additionally, one needs to know the exact amount of $d$-holes in the material, for which no straight forward experimental determination procedure exists. This drawback may be solved by either obtaining the number of $d$-holes from band structure calculations, or by expressing the sum rules results as magnetic moments per $d$-hole.

\section{ID08 and ID12 beamlines at the ESRF}

The XMCD experiments on the magnetic nanoparticles studied here were performed at the ID08 beamline (for soft X-rays ranging between 0.3 and $1.6 \mathrm{keV}$ ) in collaboration with Dr. Peter Bencok and Dr. Nick Brookes, and at the ID12 beamline (for hard X-rays ranging between 2 and $20 \mathrm{keV}$ ) in collaboration with Dr. Fabrice Wilhelm, Dr. Alevtina Smekhova and Dr. Andrei Rogalev, in the ESRF. ${ }^{9}$ These beamlines are dedicated to polarization-dependent X-ray absorption and excitation spectroscopies. Both beamlines make use of undulators to produce the polarized light for XMCD measurements. By longitudinal phasing of the magnetic arrays, the polarization of the undulator emission can be changed from linear to elliptical and circular. They complement each other to probe magnetism in a diverse range of systems with X-ray magnetooptical techniques and to study the electronic structure of materials X-ray and photoelectron emission techniques.

ID08 is equipped with two APPLE II undulators providing $\sim 100 \%$ circular/linear beam polarization, a spherical grating monochromator with an energy resolution close to $\Delta E / E=5 \times 10^{-4}$ at $850 \mathrm{eV}$, optics focussing down

\footnotetext{
${ }^{9}$ For further information about ID08 and ID12 beamlines, please visit http://www . esrf . eu/UsersAndScience/Experiments/ElectStructMagn
} 


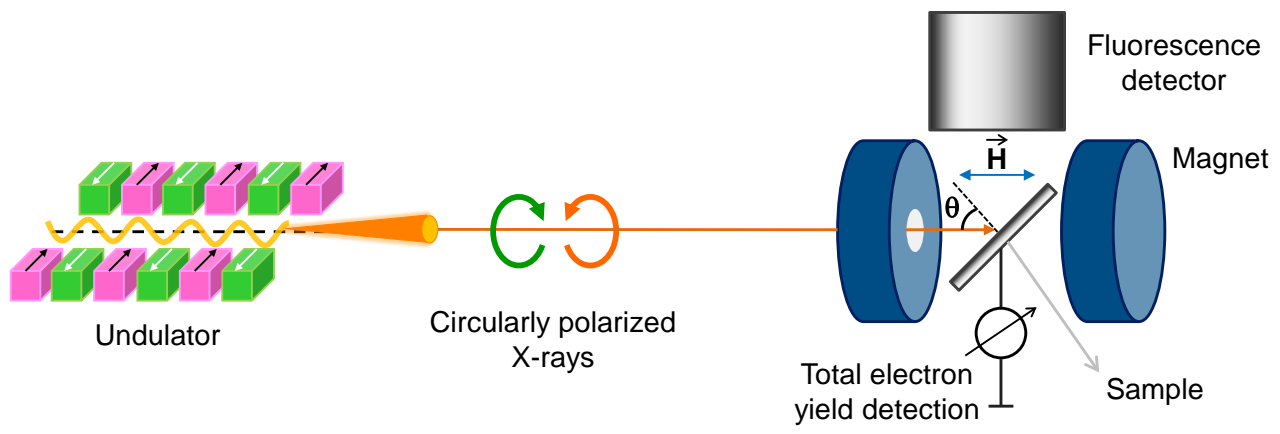

Figure 1.9: Basic diagram of the XMCD measurement setup at ID08 and ID12.

to 6-40 [60-500] microns (FWHM) vertical [horizontal] beam size, and several different UHV experimental stations and sample preparation facilities. ID12 has three helical undulators installed on the its straight section: HELIOSII, APPLE-II and the so-called "ElectroMagnet/Permanent magnet Hybrid Undulator" (EMPHU) that have been optimized to complement one another. They give ID12 the advantages of fully control of the polarization state of the incident X-ray beam over a wide energy range. ID12 uses a double-crystal monochromator with a resolution of $\Delta E / E=\sim 10^{-4}$ to select the energy of the polarized X-rays for experiments.

A schematics of the basic setup for XMCD experiments in these two beamlines is shown in Fig. 1.9. The polarized and monochromatized X-rays are directed through the sample, which is located at the center of a superconducting magnet. The magnetic field is applied on the direction of the incoming beam. The detection of the polarized dependent X-ray absorption spectra may be performed by fluorescence yield (FY) or total electron yield (TEY), the latter regularly used in the soft X-ray regime. The fluorescence detectors are (singleor multi-anode) ion implanted silicon photodiodes, which are characterized by a high efficiency in the $\mathrm{X}$-ray range, fast time response, excellent linearity, and nearly insensitivity to magnetic fields. 



\section{Chapter 2}

\section{Structural and magnetic properties of amorphous Co-W alloyed nanoparticles}

This section is devoted to investigate a system of amorphous Co- $\mathrm{W}$ alloy nanoparticles (NPs). The sequential alumina-Co-W deposition technique employed for their preparation allows to obtain self-organized arrays of particles with controlled geometries, i.e., particle sizes and interparticle distances. This potentiality has been expanded to study the magnetic properties of the amorphous Co-W NPs. In particular, we study the open question whether amorphous alloy nanoparticles may support intrinsic magnetic anisotropy, induced by alloying, irrespective of surface effects usually present in NPs systems.

Amorphous NPs constitute a different type with variable anisotropy, but few works on this field have been previously performed. Most of them are focused on Fe-based NPs [71-81] and some on Ni-based NPs [82-84], where surface effects are substantial for the blocking process and magnetic anisotropy, while, to our knowledge, no study on the mechanism of intrinsic anisotropy has been so far performed.

The election of $\mathrm{W}$ is based on the known affinity to produce $\mathrm{Co}-\mathrm{W}$ alloys by sputtering, at room temperature $[25,27,85,86\rfloor$, on the one hand, and the tendency of these alloys to become amorphous $[26-29\rfloor$, on the other hand. Besides, short range structural ordering, observed in films and bulk amorphous alloys $[87,88$, has been proposed as the mechanism inducing anisotropy in those systems. So, there was a good chance that Co-W alloying would allow varying the NPs anisotropy as a function of the tungsten concentration.

Thus, we present a systematic study of structural and magnetic proper- 
ties of the Co-W NPs system. Their size and composition are controlled by varying the amount of $\mathrm{Co}$ or $\mathrm{W}$ in the samples. The effects of these parameters on the local structure of the particles are described by analysis of highresolution transmission electron microscopy (HRTEM) and extended X-ray absorption fine structure (EXAFS) measurements; and their magnetic properties are examined by SQUID magnetometry and X-ray magnetic circular dichroism (XMCD).

\subsection{Samples studied}

The samples studied here were prepared by Dr. Cyrile Deranlot and Dr. Frederic Petroff at the Unite Mixte de Physique CNRS-Thales ${ }^{1}$ in Palaiseau, France, by sequential sputtering deposition of $\mathrm{Al}_{2} \mathrm{O}_{3}$, Co and W on a Si substrate. The deposition procedure is the same described for the Metal (M)capped Co-NPs in Ref. [11], and it is illustrated in Fig. 2.1. The cobalt, tungsten and alumina are deposited using Ar plasma, the metals in DC mode and the insulator at a RF power of $2.2 \mathrm{~W} / \mathrm{cm}^{2}$. The substrate temperature is kept constant at $293 \mathrm{~K}$ and the Ar pressure is $2 \times 10^{-3}$ Torr. The formation of Co aggregates on the amorphous alumina (see Fig. 2.1) is the result of threedimensional growth because of the different surface energies between alumina and Co [89]. Aggregation occurs below a certain threshold of the nominal thickness of $\mathrm{Co}, t_{\mathrm{Co}}$, that the layer would have if it were continuous. Previous studies show that not all the Co deposited on the alumina layer aggregates as forming the particles, but there exists a fraction of Co atoms or small clusters dispersed on the matrix, defined as $x_{\text {para }}[17\rfloor$. The values of $x_{\text {para }}$ depend on $t_{\mathrm{Co}}$, varying between 0.5 and 0.13 for $t_{\mathrm{Co}}=0.4$ and $1.0 \mathrm{~nm}$, respectively [17. The Co clusters are subsequently capped with a $\mathrm{W}$ layer, whose nominal thickness is denoted by $t_{\mathrm{W}}$. A new alumina layer of about $3 \mathrm{~nm}$ is deposited on top of this Co-W system. At the end of the deposition process, a final thicker alumina layer of $5 \mathrm{~nm}$ is deposited for protection purposes. This sequential deposition process is repeated $\mathrm{N}$ times in order to get a multilayer system, following the formula $\mathrm{Al}_{2} \mathrm{O}_{3} /\left(\mathrm{Al}_{2} \mathrm{O}_{3} / \mathrm{Co} / \mathrm{W}\right)_{\mathrm{N}} / \mathrm{Al}_{2} \mathrm{O}_{3}$.

The samples measured may be grouped into two series: $a$, those with $t_{\mathrm{Co}}$ fixed at $0.7 \mathrm{~nm}$ and varying $\mathrm{W}$ capping layer, $t_{\mathrm{W}}=0.6,1.5$ and $4.5 \mathrm{~nm}$, and $b$, with constant capping layer $t_{\mathrm{W}}=1.5 \mathrm{~nm}$ and varying $t_{\mathrm{Co}}=0.4,0.7$ and $1.0 \mathrm{~nm}$. Most of the samples have $\mathrm{N}=25$, except that with $t_{\mathrm{Co}}=1.0 \mathrm{~nm}$ and $t_{\mathrm{W}}=1.5 \mathrm{~nm}$, for which $\mathrm{N}=20$.

A bulk $\mathrm{Co}_{3} \mathrm{~W}$ alloy has been used in the present study as a reference

\footnotetext{
${ }^{1}$ http://www.trt.thalesgroup.com/ump-cnrs-thales/
} 


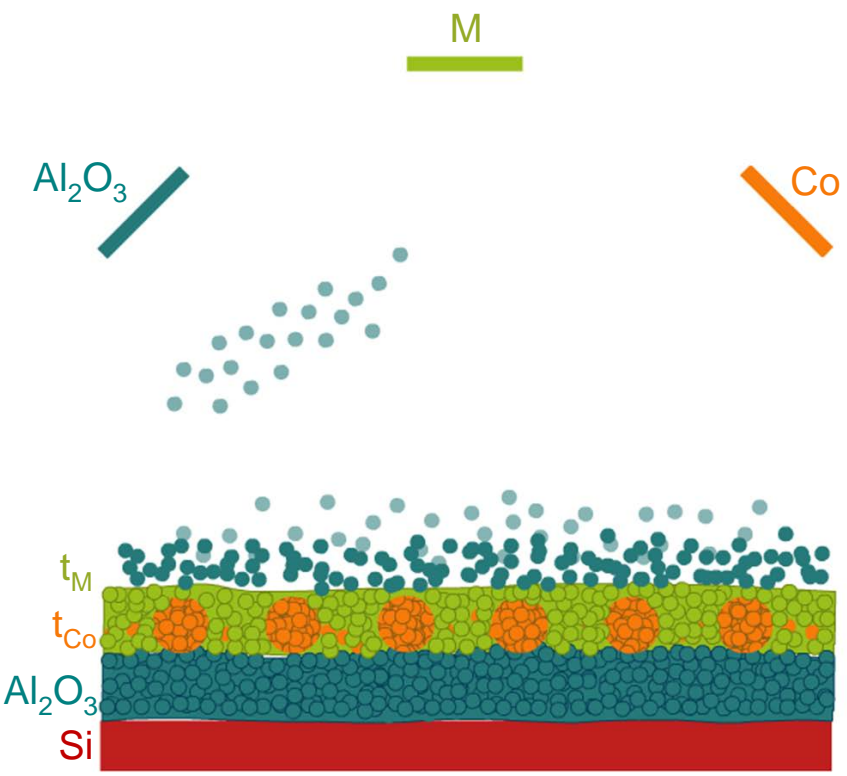

Figure 2.1: Illustration of the sequential sputtering deposition process of the Co-M NPs.

sample. It has been synthesized by melting the pure metallic precursors in an induction furnace under Ar atmosphere. The encapsulated sample was thermally treated for seven days at a temperature of $1300 \mathrm{~K}$ in a muffle furnace. After the annealing, the sample was quenched by immersing the quartz tube in room temperature water.

The chemical composition of the bulk $\mathrm{Co}_{3} \mathrm{~W}$ alloy was confirmed by means of energy-dispersive X-ray spectroscopy (EDX) performed by an Oxford Instruments INCA $300 \mathrm{X}$-Sight as part of a JEOL JSM 6400 scanning electron microscope (SEM) in the Servicio de Microscopía Electrónica de Materiales of the Universidad de Zaragoza. ${ }^{2}$ The crystalline structure of the alloy was determined by X-ray powder diffraction (XRD) measurements performed at room temperature using a rotating anode D-Max Rigaku diffractometer. XRD data were collected in a range $20^{\circ}<2 \theta<80^{\circ}$ using $\mathrm{Cu} K_{\alpha}$ radiation, and are shown in Fig. 2.2. The $\mathrm{Co}_{3} \mathrm{~W}$ alloy is an $h c p$ phase with spatial group $\mathrm{P}_{3} m c$. A profile matching (PM) routine was run on this data using FULLPROF suite code $[90$. The position of the diffraction peaks in the XRD profile are well matched with this routine using the $\mathrm{Co}_{3} \mathrm{~W}$ crystallographic information taken from the ICSD ${ }^{3}$ database as initial parameters (see Fig. 2.2). The lattice parameters obtained from the $\mathrm{PM}$ for the bulk $\mathrm{Co}_{3} \mathrm{~W}$ alloy were $a=b=5.144$ $\AA$ and $c=4.141 \AA$, very close to those of the $\mathrm{Co}_{3} \mathrm{~W}$ listed in the ICSD. How-

\footnotetext{
${ }^{2}$ http://sai.unizar.es/microscop-mat/

${ }^{3}$ http://icsd.iqfr.csic.es/icsd/
} 


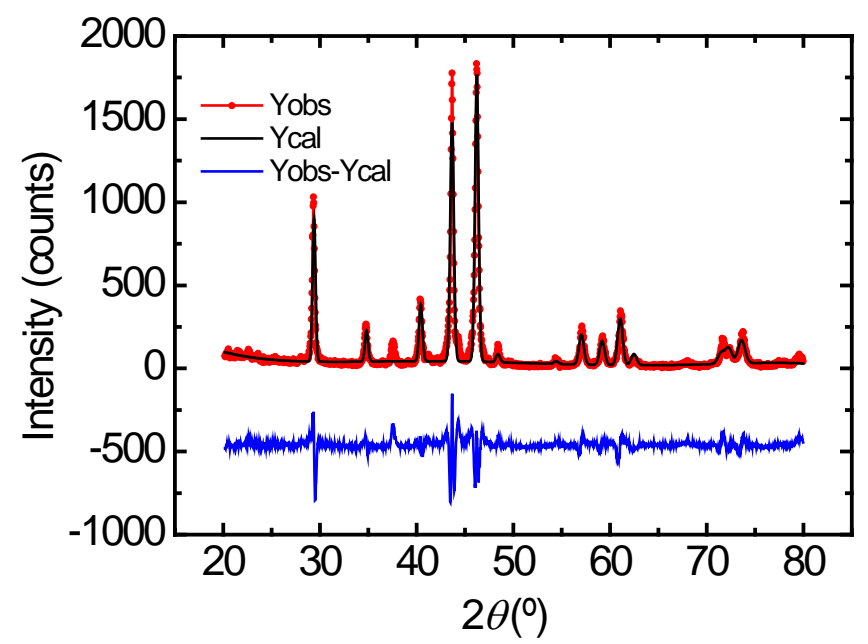

Figure 2.2: $\mathrm{XRD}$ data and $\mathrm{PM}$ results of the bulk $\mathrm{Co}_{3} \mathrm{~W}$ alloy.

ever, the structure in the bulk $\mathrm{Co}_{3} \mathrm{~W}$ sample is found to be rather disordered, which is evident from the broad and less intense diffraction peaks observed, compared to regular highly crystalline materials, and from the mismatch of the peaks width in the PM results. Thus, a complete Rietveld analysis on this dataset was not possible. There are a very few diffraction peaks not identified by the PM routine that may be assigned to the $\mathrm{Co}_{7} \mathrm{~W}_{6}$ phase; such phase has also been observed in the SEM and EDX study, but it has been estimated to be less than $2 \%$ in the sample.

\subsection{Morphological and structural study}

\subsubsection{Microscopy Measurements}

HRTEM measurements were performed by Dr. Luisa Ruiz González and Dr. José María González-Calbet of the Departamento de Química Inorgánica in the Universidad Complutense de Madrid. ${ }^{4}$ HRTEM images were taken on both $a$ and $b$ samples series in cross section configuration, in a JEOL JEM 3000 F Field Emission Microscope fitted with an Oxford LINK Electron Dispersive Spectrometry (EDS) analyzer. Cross section specimens were prepared by conventional mechanical thinning and ion milling techniques.

HRTEM and high angle annular dark field (HAADF) scanning transmission electron microscopy (STEM) measurements were performed on two samples in plan view configuration by Dr. Cesar Magén and Dr. Alfonso Ibarra in

\footnotetext{
${ }^{4}$ http://www. cnme.es/
} 
a FEI Tecnai ${ }^{\text {TM }}$ G2 F30 STWIN Field Emission Microscope of the Laboratorio de Microscopías avanzadas, Instituto de Nanociencia de Aragón, Universidad de Zaragoza. ${ }^{5}$ Samples for plan-view observation were prepared by deposition of a $\mathrm{Al}_{2} \mathrm{O}_{3} / \mathrm{Co} / \mathrm{W} / \mathrm{Al}_{2} \mathrm{O}_{3}$ sandwich on TEM carbon grids, with $t_{\mathrm{Co}}=0.7 \mathrm{~nm}$ and $t_{\mathrm{W}}=0.6$ and $1.5 \mathrm{~nm}$.

Low magnification studies in cross section configuration for all five samples confirmed the presence of 25 layers (20 layers in the case of $t_{\mathrm{Co}}=1.0 \mathrm{~nm}$ and $t_{\mathrm{W}}=1.5 \mathrm{~nm}$ ) in agreement to the nominal layer preparation. HRTEM images and EDS analysis for both samples series (Fig. 2.3 and 2.4) indicate that light gray layers correspond to alumina and darker layers to metal (Co and $\mathrm{W}$ ). These layers exhibit a washboard cross sectional shape. In stark contrast to Co-nanoparticles systems covered with a noble metal layer, where the crystal structure of the Co particle is not affected by the capping [11], W alloys with Co in those places where the Co NPs were originally formed. By inspection of the particles in Fig. 2.3(a) and 2.3(b), there seems to be very short range order within each layer due to the higher $\mathrm{Co} / \mathrm{W}$ ratio in these two samples, but this is neither detected by SAED (Selected Area Electron Diffraction), nor by Fourier transform. In fact, the SAED images show the typical halo diffraction pattern corresponding to an amorphous structure (see Fig. 2.4(d)). Regarding this feature, some authors suggest that amorphous Co-W films consist of tetrahedral units composed of one $\mathrm{W}$ and three Co atoms, which arrange in a structure that does not exhibit macroscopic crystal symmetry [91]. Others ascribe this short range ordering (SRO) in amorphous alloys to the existence of icosahedral and octahedral clusters [92 . Some of these structures are probably being formed in our Co-W NPs. We will further discuss this topic in section 2.2.2.

The Co-W amorphous particles in series $a$ and $b$ are more detached in samples with higher $\mathrm{Co} / \mathrm{W}$ ratio, as in that of $t_{\mathrm{Co}}=0.7 \mathrm{~nm}$ and $t_{\mathrm{W}}=0.6 \mathrm{~nm}$ (Fig. 2.3(a)). In those samples where the $\mathrm{Co} / \mathrm{W}$ ratio decreases, $\mathrm{W}$ tends to join the particles by filling the interparticle spaces (Fig. 2.3(b) and 2.3(c)). These particles with elongated cross sectional shape arrange within layers in a periodical way, similarly to their assembly with alumina capping [89]. That is, in each layer, particles lay on the depression formed by the previous deposited particles layer so that they form a hexagonal arrangement across the layers, as it is marked in Fig. 2.3(a). All this morphology described for samples from the $a$ series has also been observed for samples from the $b$ series (see Fig. 2.4).

Structural differences are found in the $a$ series sample with $t_{\mathrm{W}}=4.5 \mathrm{~nm}$. As it is observed in a HRTEM image for this sample (Fig. 2.3(c)), the interparticle spaces existing in all other four samples have been filled, so that

\footnotetext{
${ }^{5}$ http://ina.unizar.es/lma/index.html
} 


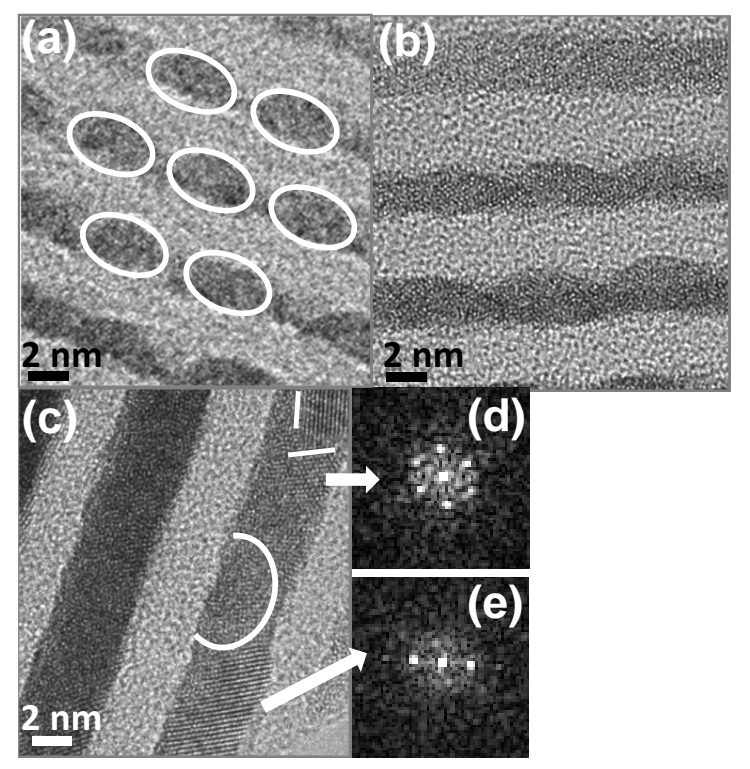

Figure 2.3: HRTEM images in cross section configuration for samples from the $a$ series with $t_{\mathrm{Co}}=0.7 \mathrm{~nm}$ and (a) $t_{\mathrm{W}}=0.6 \mathrm{~nm}$, (b) $t_{\mathrm{W}}=1.5 \mathrm{~nm}$ and (c) $t_{\mathrm{W}}=4.5 \mathrm{~nm}$. Fourier transforms (FFTs) at selected zones of the image in (c) are shown in (d) and (e). Arrangement of the particles within layers is highlighted in (a). An amorphous zone on one of the layers of sample in (c) is demarcated by a white line.

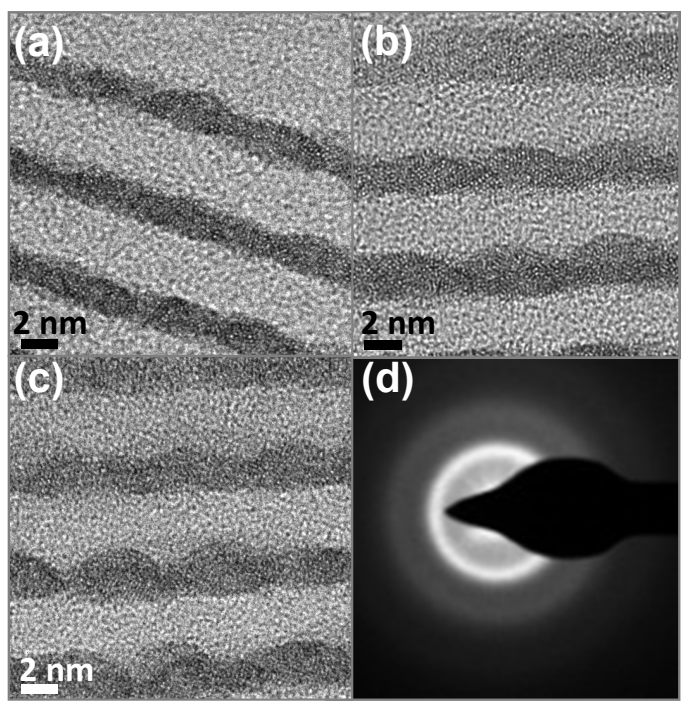

Figure 2.4: HRTEM images in cross section configuration for samples from the $b$ series with $t_{\mathrm{W}}=1.5 \mathrm{~nm}$ and (a) $t_{\mathrm{Co}}=0.4 \mathrm{~nm}$, (b) $t_{\mathrm{Co}}=0.7 \mathrm{~nm}$ and (c) $t_{\mathrm{Co}}=1.0$ $\mathrm{nm}$. (d) SAED image of a NP in sample in (b). 
the $\mathrm{Co} / \mathrm{W}$ film has a nearly constant thickness. Each metallic layer of about $4.2 \mathrm{~nm}$ thick, shows ordered zones alternating with amorphous zones. In the ordered regions, periodicity is around $0.2 \mathrm{~nm}$, confirmed by fast Fourier transform (FFT) performed on some of the ordered zones (see bright spots observed in Fig. 2.3(d) and 2.3(e)). This periodicity and the distances and angles on the FFT are in agreement with the $\left(\begin{array}{lll}1 & 1 & 0\end{array}\right)$ planes of $\mathrm{W}$ bcc structure. There is also evidence of different grains within the crystalline zones on the layer, identified, for example, by the two orientation of the planes observed in the HRTEM image (see Fig. 2.3(c)), and the splitting of the spots in Fig. 2.3(d). EDS analysis show the presence of both Co and W. However, it is not easy to establish differences between ordered and disordered zones by this technique. Given the higher amount of $\mathrm{W}$ in this last sample, the Co- $\mathrm{W}$ alloy is possibly saturated, so that $\mathrm{W}$ starts to generate the crystalline zones identified from the images (Fig. 2.3(c)). Further discussion about W growing as a crystal in the samples is presented in the EXAFS section (see section 2.2.2).

High contrast obtained in plan view HAADF STEM images for the $a$ series samples with $t_{\mathrm{Co}}=0.7 \mathrm{~nm}$ and $t_{\mathrm{W}}=0.6$ and $1.5 \mathrm{~nm}$ (Fig. 2.5(a) and 2.5(b)) allows easier identification of the particles than in HRTEM images due to the huge $\mathrm{Z}$ contrast between the heavy metal particles and the light oxide matrix. In these images, brighter parts correspond to Co-W nanoparticles, and darker parts to $\mathrm{Al}_{2} \mathrm{O}_{3}$. Images obtained are comparable to those of similar systems of metallic nanoparticles embedded in insulator matrices [93], and the shape of the particles is similar to that of Co-nanoparticles embedded in alumina [94]. HAADF STEM images reveal that particles in sample with $t_{\mathrm{W}}=1.5 \mathrm{~nm}$ (Fig. $2.5(\mathrm{~b}))$ are bigger and more closely packed than particles in sample with $t_{\mathrm{W}}=$ $0.6 \mathrm{~nm}$ (Fig. 2.5(a)).

Size distributions for samples from the $a$ series with $t_{\mathrm{Co}}=0.7 \mathrm{~nm}$ and $t_{\mathrm{W}}=0.6 \mathrm{~nm}$ (Fig. 2.5(c)) and $1.5 \mathrm{~nm}$ (Fig. 2.5(d))were obtained by digital processing of the images taken, for both cross section (Fig. 2.3(a) and 2.3(b)) and plan view configurations (Fig. 2.5(a) and 2.5(b)). Results for the diameter distribution on each case were fitted using a Gaussian distribution function. The values obtained from this analysis are listed in table 2.1. The effective diameters for each configuration, $\left\langle D_{\text {eff }}\right\rangle_{\mathrm{CS}}$ for Cross Section and $\left\langle D_{\text {eff }}\right\rangle_{\mathrm{PV}}$ for Plan View, were obtained with the assumption that particles have circular in-plane and elliptic out-of-plane projection shape, the latter with short axis $a$ and long axis $b$ on each projection, so that $D_{\text {eff }}=\sqrt{a b}$. Interparticle distance, $d$, and surface density of particles, $N_{S}$, expressed as number of particles per $\mathrm{nm}^{2}$ for the plan view images, were also obtained (see table 2.1 ). These two values are almost equal for both samples analyzed in plan view, which means that particles are spatially distributed on each layer in the same way for those samples from the $a$ series. Cross section values have a higher error since they 

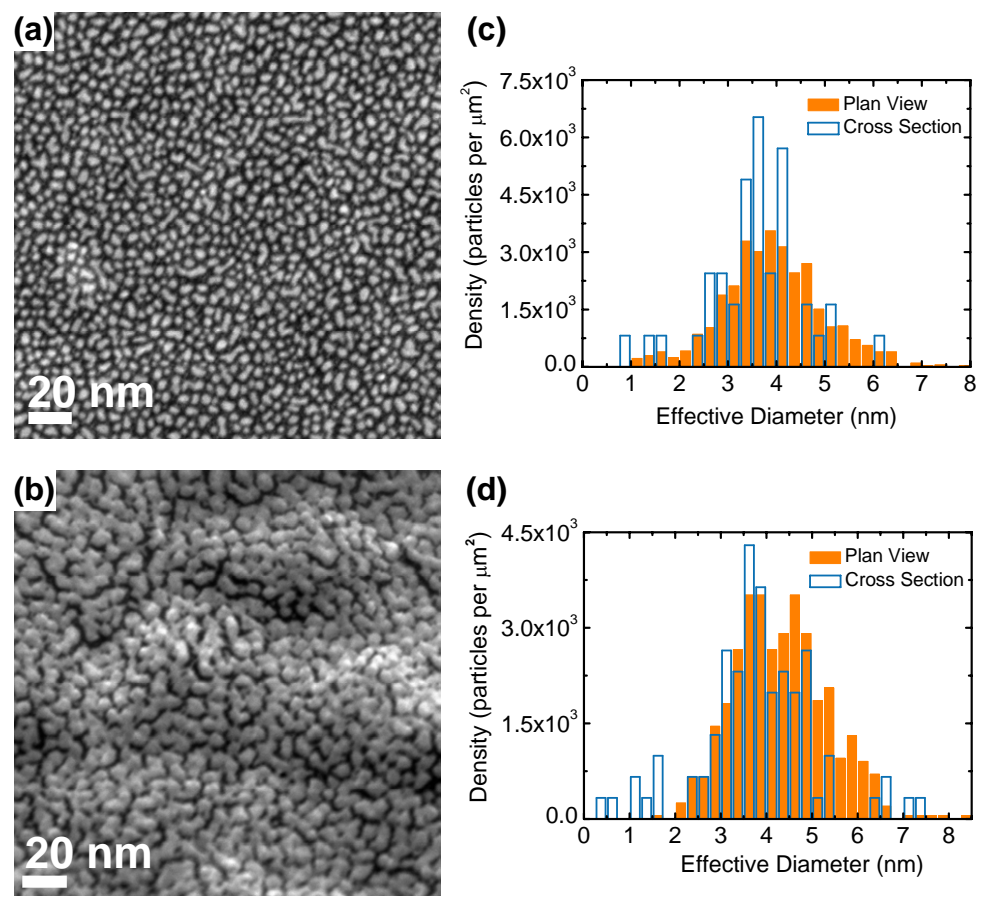

Figure 2.5: HAADF STEM images in plan view configuration for the $a$ series samples with $t_{\mathrm{Co}}=0.7 \mathrm{~nm}$ and (a) $t_{\mathrm{W}}=0.6 \mathrm{~nm}$ and (b) $t_{\mathrm{W}}=1.5 \mathrm{~nm}$. Size distribution of plan view and cross section configurations for the samples in (a) and (b) are shown in (c) and (d), respectively.

were obtained from poor contrast HRTEM images, so particle identification was less precise than in the HAADF STEM images in the plan view case.

Size distributions for cross section and plan view configurations for the $a$ samples series are shown in Fig. 2.5(c), for sample with $t_{\mathrm{W}}=0.6 \mathrm{~nm}$, and in Fig. $2.5(\mathrm{~d})$ for sample with $t_{\mathrm{W}}=1.5 \mathrm{~nm}$, both with $t_{\mathrm{Co}}=0.7 \mathrm{~nm}$. These distributions are centered near the same mean $\left\langle D_{\text {eff }}\right\rangle$; i.e., there is a slight difference between both values. $\left\langle D_{\text {eff }}\right\rangle$ for the in-plane projection shape obtained from the plan view configuration analysis is larger than the out-ofplane projection from cross section analysis in both samples $\left(t_{\mathrm{W}}=0.6\right.$ and 1.5 $\mathrm{nm})$. Thus, Co-W particles have an oblate shape.

Digital analysis of the images obtained in cross section for samples from the $b$ series were also performed in order to identify some morphological parameters; however, size distributions in these cases were not obtained, due to the low contrast and less separation between the particles in these two samples (see Fig. 2.4(a) and 2.4(c)). A clear tendency in the size of the particles given by the $\mathrm{Co} / \mathrm{W}$ ratio in the samples is also evident from the values in table 2.1 , so that out-of-plane projection increases from 2.84 to $4.24 \mathrm{~nm}$ for samples in 


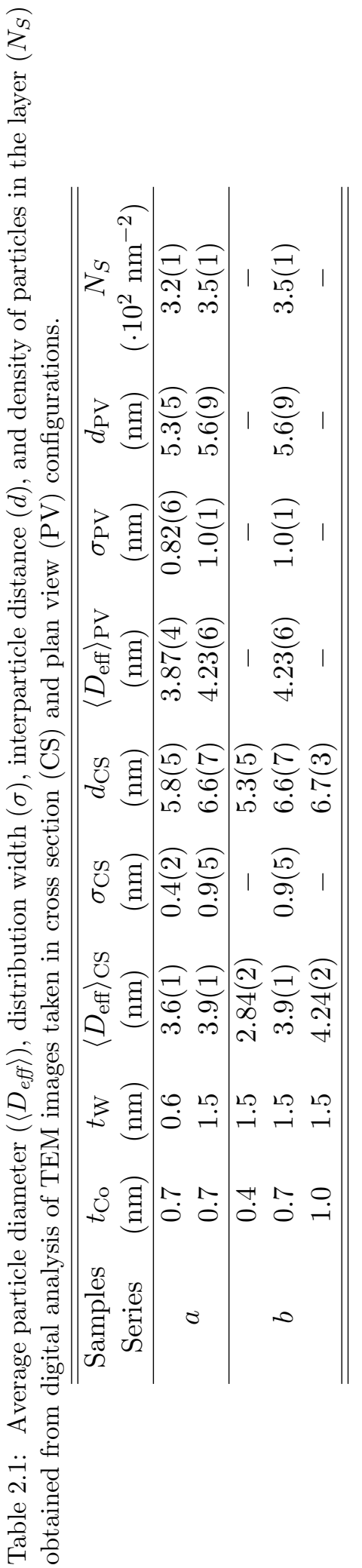


the $b$ series, as $t_{\text {Co }}$ increases from $0.4 \mathrm{~nm}$ to $1.0 \mathrm{~nm}$.

The elemental composition of the nanoparticles has been measured by EDS on the plan view sample with $t_{\mathrm{Co}}=0.7 \mathrm{~nm}$, and $t_{\mathrm{W}}=0.6 \mathrm{~nm}$. A total of 12 particles were measured yielding to an average composition with tungsten content of $27 \pm 6$ at. $\% \mathrm{~W}$; i.e. the particle composition is close to that of $\mathrm{Co}_{3} \mathrm{~W}$. Moreover, the composition within one particle was constant within experimental error. The relative variation in the composition of the other particles has been determined by EXAFS analysis at the Co $K$-edge (see section 2.2.2).

From this morphological study we conclude that we have obtained a system of self-organized and uniformly dispersed amorphous Co-W alloy NPs. This occurs since W has alloyed with the preformed Co NPs, instead of growing as a homogeneous crystalline film covering the particles. The size of the amorphous particles depends on the amount of Co and $\mathrm{W}$ deposited on each layer. Particles formed within the layers have an oblate shape. When the amount of $\mathrm{W}$ increases and the Co-W alloy saturates, $\mathrm{W}$ grows as a crystal and fills the interparticle spaces.

Previous works on Co-W thin films have led to similar amorphous structure, when such films are synthesized by sputtering, using composite targets $\lfloor 26,95\rfloor$. The amorphous forming ability (AFA) of Co-W has been studied in terms of the Miedema's coordinates model, $Y=\left|\Delta \phi / \Delta n_{\mathrm{WS}}^{1 / 3}\right|$, where $\Delta \phi=\phi_{\mathrm{Co}}-\phi_{\mathrm{W}}$, and $\Delta n_{\mathrm{WS}}=n_{\mathrm{WS}}(\mathrm{Co})-n_{\mathrm{WS}}(\mathrm{W})$, where $\phi$ is the chemical potential, and $n_{\mathrm{WS}}$ the electron density of the Wigner-Seitz unit cell, and $Z=\left|\left(R_{\mathrm{W}}-R_{\mathrm{Co}}\right) / R_{\mathrm{Co}}\right|\lfloor 95\rfloor$. The binary alloy phase space is separated by the empirical law $Y=2.52 Z^{-1 / 4}$ in the amorphous and crystalline areas. It was found that Co-W lies in the amorphous region; the reason for this favorable AFA in this alloy is a negative heat of mixing, large atomic size differences and a low substrate temperature that avoids recrystallization via diffusion. These factors have explained satisfactorily the formation of $\mathrm{Co}-\mathrm{W}$ amorphous thin films by co-sputtering $\lfloor 26\rfloor$. In our preparation method Co nanoparticles are formed first, and a subsequent W capping supplies the alloying metal. The sputtering voltage is enough to let $\mathrm{W}$ and Co react, while the substrate temperature avoids crystallization. The AFA is high since the W concentration is higher that $17 \%$, a condition needed for the formation enthalpy of the amorphous phase to be lower than the crystalline one $[95$. Therefore, amorphization is favorable in the formation of Co-W NPs, just as it is in the thin film case. 


\subsubsection{XANES and EXAFS measurements}

X-ray absorption near edge structure (XANES) and EXAFS spectra for both $a$ and $b$ samples series were measured at the Co $K(7709 \mathrm{eV})$ and $\mathrm{W} L_{3}(10207$ $\mathrm{eV}$ ) edges using a double-crystal Si(111) monocromator in the BM29 beamline at the European Synchrotron Radiation Facility (ESRF). Measurements were performed at $300 \mathrm{~K}$ in fluorescence detection mode with a 13-element Ge solid state detector with digital signal processing for fluorescence X-ray absorption spectroscopy (XAS), high energy resolution and high count rate. Reference samples were also measured at different edges: Co particles embedded in alumina matrix (referred in the following as "bare Co NPs") $\left(\mathrm{Al}_{2} \mathrm{O}_{3} / \mathrm{Co}\right)_{20}$ with $t_{\mathrm{W}}=0.7 \mathrm{~nm}$ measured at the $\mathrm{Co} K$ edge, $\mathrm{Co}_{3} \mathrm{~W}$ alloy measured at the Co $K$ and $\mathrm{W} L_{3}$ edges, Au capped particles with formula $\left(\mathrm{Al}_{2} \mathrm{O}_{3} / \mathrm{Co} / \mathrm{Au}\right)_{25}, t_{\mathrm{Au}}=$ 1.5 and $4.5 \mathrm{~nm}$, measured at the $\mathrm{Co} K$ and $\mathrm{Au} L_{3}$ edges. The reference foils: Co metallic foil measured at the Co $K$ edge, W metallic foil measured at the $\mathrm{W} L_{3}$ edge and $\mathrm{Au}$ foil measured at the $\mathrm{Au} L_{3}$ edge. For each edge measured on each sample, four spectra were taken in order to have enough statistics in data treatment, following a $4 \mathrm{~s} /$ point count.

Some of the XANES spectra at the $\mathrm{W} L_{3}$ edge were recorded at the ESRF ID12 beamline, by a fluorescence detector in backscattering geometry. The APPLE-II undulator and a double - Si - (111) - crystal monocromator were used to collect the spectra.

The X-ray absorption spectra were analyzed following standard procedures. A preliminary reduction of the EXAFS raw data involving background removal of the absorption data $\mu(\mathrm{E})$, conversion of $\mu(\mathrm{E})$ to $\chi(\mathrm{k})$, normalization and weighting scheme were performed with AUTOBK and ATHENA, programs from the simulation package IFEFFIT [96]. EXAFS data analysis and fitting were performed on all reference and samples at their respective edges. All spectra were fitted using the ARTEMIS program from the same IFEFFIT package using models based on the crystallographic information found in the ICSD $^{6}$ database. Atomic clusters used to generate the scattering paths for fitting were generated with ATOMS, a tool from the same simulation package $[97\rfloor$.

\section{Co $K$ edge results}

The X-ray absorption near edge structure (XANES) allows to study the electronic structure of Co (electron configuration $[\mathrm{Ar}] 3 d^{7} 4 s^{2}$ ) and $\mathrm{W}$ (electron configuration $\left.[\mathrm{Xe}] 4 f^{14} 5 d^{4} 6 s^{2}\right)$ atoms of the $\mathrm{Co}-\mathrm{W}$ systems. Spectra at Co $K$

\footnotetext{
${ }^{6}$ http://icsd.iqfr.csic.es/icsd/
} 

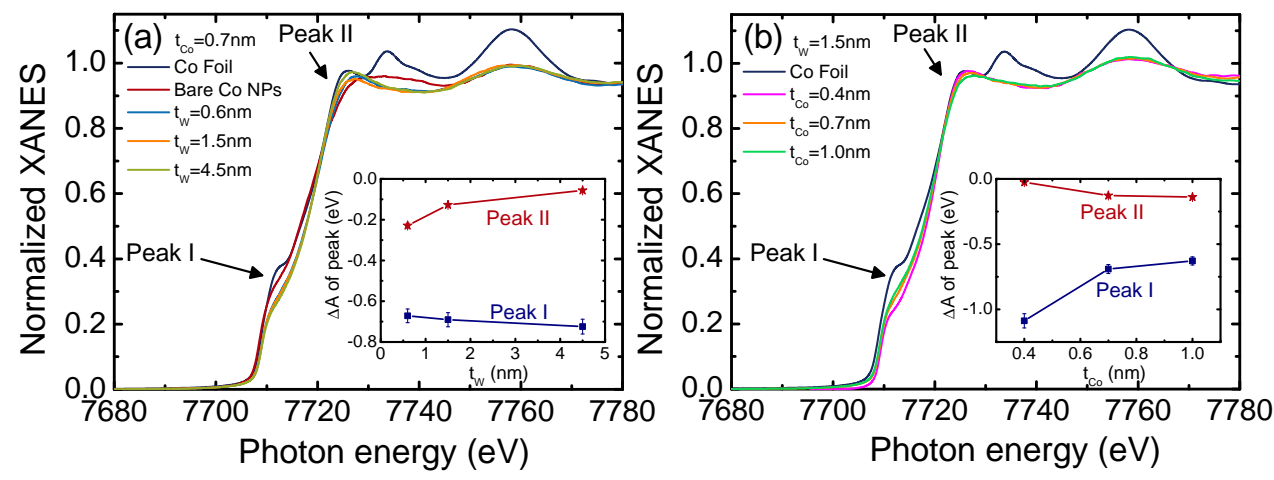

Figure 2.6: XANES at the Co $K$ edge for the Co-W NPs (a) $a$ samples series, (b) $b$ samples series. A spectrum for the Co foil is plotted for reference in both cases. In figure (a) a curve for bare Co NPs with $t_{\mathrm{Co}}=0.7 \mathrm{~nm}$ has been included for comparison. Insets: Variation of the areas of peak I and peak II in figure (a) and (b) as a function of $t_{\mathrm{W}}$ and $t_{\mathrm{Co}}$, respectively.

edge for Co-W NPs from the $a$ and $b$ series are shown in Fig. 2.6(a) and 2.6(b), respectively. In both cases a Co foil XANES is plotted for reference, and a spectrum for bare Co NPs with $t_{\mathrm{Co}}=0.7 \mathrm{~nm}$ is plotted in Fig. 2.6(a) for comparison. Changes in the intensity of the pre-edge peak and the white line (denoted as peak I and peak II, respectively, in Fig. 2.6) are noticeable. The variation of the peaks areas $\left(\Delta \mathrm{A}=\int_{E_{i}}^{E_{f}}\left(\mu_{\text {sample }}-\mu_{\text {Co-foil }}\right) d E\right)$ in series $a$ and $b$ is shown in the insets of Fig. 2.6(a) and 2.6(b), respectively. The integration limits were $7708.4-7721.3 \mathrm{eV}$ for peak I, and $7721.3-7729 \mathrm{eV}$ for peak II.

The Co $K$ edge white line reflects its $4 p$ empty states near the Fermi energy. The origin of peak $\mathrm{I}$ is at the density of states (DOS) of $d$-character available at the Fermi energy, projected onto the Co site $[98,99\rfloor$. This is influenced by intra-atomic Co $4 p-3 d$ hybridization, as it will be further described in section 3.1.1. Moreover, in our samples, this $d$-DOS originates both at the Co $3 d$ band and at the $\mathrm{W} 5 d$ states through interatomic Co $3 d-\mathrm{W} 5 d$ hybridization. The intensity of peak I in Co-W NPs is clearly lower than in metal cobalt, and than bare Co NPs for the $a$ samples series. The same reduction at peak I with respect to Co foil is observed for the $b$ series. Although alloying with $\mathrm{W}$ introduces a higher number of $d$ holes in the system, hybridization strongly delocalizes the $d$ empty states, depleting the intensity of peak I by reducing the $d$-DOS projected at the Co site. Therefore, the strong reduction of peak $\mathrm{I}$ at the Co $K$ edge of Co-W NPs is a proof of Co $4 p-3 d-\mathrm{W} 5 d$ hybridization.

In series $a$, peak I is practically constant along the series (see inset of Fig. $2.6(\mathrm{a}))$, reflecting that $\mathrm{Co} 3 d-\mathrm{W} 5 d$ hybridization is not affected by small variations in the amount of $\mathrm{W}$ for a fixed Co content. This, in principle, 
surprising fact strongly suggests that the alloy composition is similar for the three samples along this series, a result that will be confirmed by EXAFS (later in this section) and by magnetic analysis (section 2.3.1). In contrast, $b$ samples series show a clear increase of the $\Delta \mathrm{A}$ of peak I upon increasing Co content in the NPs, as shown in the inset of Fig. 2.6(b). This trend is the expected one, as a decrease in the proportion of $\mathrm{W}$ (or increase of $\mathrm{Co} / \mathrm{W}$ ratio) in the sample lowers the number of $5 d$ holes available for hybridization, increasing the localization of the $d$-character states at the Co site.

The intensity of peak II depends on both the number of available $4 p$ states, and the structure of the system, in a non-trivial way. The comparison of the Co metal and Co-W NPs has to be taken with care, as their structures are different ( $h c p$ and amorphous, respectively). However, the trend of $\Delta \mathrm{A}$ of peak II in samples from the $a$ series indicates that the number of $4 p$ holes is enhanced as the $\mathrm{W}$ content increases, suggesting an electronic transfer from Co $4 p$ towards W $5 d$ states. Accordingly, $\Delta \mathrm{A}$ of peak II in samples from the $b$ series slightly decreases with Co content, suggesting that, for the range of $t_{\mathrm{Co}}$ and $t_{\mathrm{W}}$ studied, the number of $4 p$ holes is reduced as the ratio $\mathrm{Co} / \mathrm{W}$ increases. In short, along the $a$ and $b$ series, as the $\mathrm{Co} / \mathrm{W}$ ratio increases, both the $4 p$ number of empty states and the delocalization of $3 d$ empty states decrease.

Structural analysis that can be extracted from the $\chi(\mathrm{k})$ EXAFS signal is better studied by its Fourier transform, shown in Fig. 2.7, performed on a krange from 2.0 to $11.9 \AA^{-1}$ using a $\mathrm{k}^{2}$ weight and a hanning window function. This $R$-space data show peaks which correspond to different atomic shells surrounding the absorbing atom, Co in this case. In Fig. 2.7(a), it can be seen that the position and shape of the peaks in the bare Co nanoparticles and Co foil are very similar, not only for the first coordination shell, but also for outer shells, showing that Cobalt in $\mathrm{Co} / \mathrm{Al}_{2} \mathrm{O}_{3}$ aggregates in a similar structure as metallic Co, as it has been reported in the literature $[94\rfloor$. However, the peaks for outer coordination shells are different in the W-capped Co nanoparticles. This is caused by a change in the Co coordination due to alloying between Co-W, which is also evident in Fig. 2.7(b) for the $b$ sample series. Indeed, intensities of all peaks in both Fig. 2.7(a) and 2.7(b) are larger in the Co foil than in the particulate systems, as it is expected from the reduction in the mean Co coordination due to the size reduction.

In the present study, EXAFS signal for the bare Co nanoparticles with $t_{\mathrm{W}}=0.7 \mathrm{~nm}$ was fitted using a Co - $f c c$ metal model, where the coordination number $N$ was used as a fitting parameter. A reduction from $N=12$ in the metallic Co to $N=6.1$ in the first coordination shell of the bare Co nanoparticles was obtained from this fit. However, recent works have demonstrated that such a strong reduction in the number of first neighbors could be 


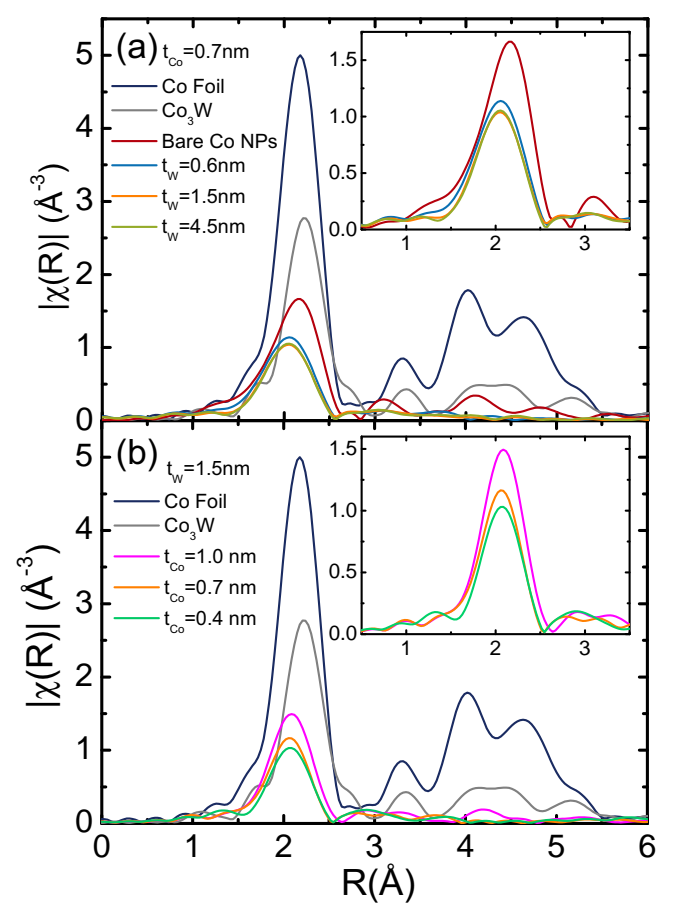

Figure 2.7: Fourier transform of EXAFS signal at the Co $K$ edge on the Co-W samples. (a) a samples series. (b) $b$ samples series. Measurements for Co foil and bulk $\mathrm{Co}_{3} \mathrm{~W}$ in both cases, and bare Co nanoparticles with $t_{\mathrm{Co}}=0.7 \mathrm{~nm}$ in (a) are shown for comparison. Inset: zoom of the first coordination shell in the particulate systems.

an artifact of EXAFS analysis [100, due to effects of surface distortions on the nanoparticles, producing an underestimation of the average coordination number.

A reduction in the EXAFS signal as a result of lower Co coordination due to alloying is also observed when the signal for the Co foil is compared with the one for bulk $\mathrm{Co}_{3} \mathrm{~W}$ in Fig. 2.7(a) and 2.7(b). This behaviour supports the scheme of $\mathrm{Co}-\mathrm{W}$ alloy presence in the $\mathrm{Co}-\mathrm{W}$ nanoparticles. There is a further reduction in the amplitude of all peaks and lack of structure beyond the first two coordination shells in the Co-W nanoparticles systems, which is characteristic of amorphicity, thus supporting the observation of amorphous structure in the HRTEM measurements. In general, amorphous compounds are characterized by damping of their EXAFS signal compared to a reference foil, consistent with static disorder and reduction of average coordination number [101].

In the HRTEM section we have mentioned that capping the Co nanoparticles with noble metals does not affect the structure of the NPs $[11\rfloor$. This has 


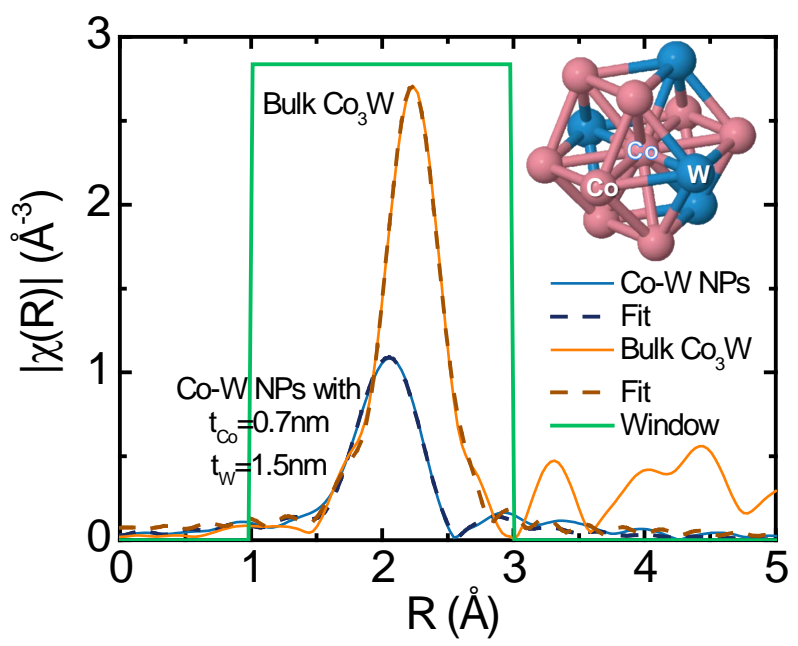

Figure 2.8: $\quad$ FT EXAFS signal at the $\mathrm{Co} K$ edge for the bulk $\mathrm{Co}_{3} \mathrm{~W}$ alloy and comparison with that of a Co-W alloy NPs sample. Dashed lines: best fit of the first Co coordination shell for each sample. Inset: an illustration of the structure of a Co first coordination shell.

also been observed with EXAFS, so that bare and Au-capped Co nanoparticles have equivalent $f c c$ crystal structure when comparing their Fourier transformed EXAFS signal ${ }^{7}$. However, capping the Co NPs with W completely modifies the structure of the original particles by forming an amorphous alloy.

Different models were tested in order to fit the Co $K$ edge $R$-space data for the $\mathrm{W}$-capped Co nanoparticles. Some of the fitting tests were performed by using a model of pure Co - $f c c$ metal, such as the one typically used in the bare Co nanoparticles case, and by using a W - doped metal Co model. However, the best fitting model was obtained by using the Co - scattering paths of the most stable $\mathrm{Co}-\mathrm{W}$ alloy, ${ }^{8}$ i.e., $\mathrm{Co}_{3} \mathrm{~W}$, and determining the deviation in the average coordination $(N)$ and interatomic distance $(R)$ for each Co-W NPs sample.

Given the high affinity of $\mathrm{Co}$ and $\mathrm{W}$ to form Co-W alloys $[25,27,85,86\rfloor$ we have assumed that all Co in the Co-W NPs samples is uniformly alloyed

\footnotetext{
${ }^{7}$ EXAFS spectra for the Au-capped Co NPs were recorded during the same experiment and compared to those for the uncapped Co-NPs, both with the same $t_{\mathrm{Co}}=0.7 \mathrm{~nm}$. We found that both systems have $f c c$ structure and very close first near neighbor distances. Their Fourier Transformed EXAFS profiles are almost identical, even for outher coordination shells. The first coordination shell peak in the Co - Au NPs is higher in amplitude than the uncapped ones and it increases with the content of $\mathrm{Au}$ in the sample due to the high crystallinity in these noble - metal capped particles. Then, contrary to the case of $\mathrm{W}$ deposit, noble metals - capping do not modify the crystal structure of the Co NPs.

${ }^{\varepsilon}$ http://www.sgte.org/
} 
with $\mathrm{W}$. Then, we have estimated the change of the Co environment when increasing the amount of $\mathrm{W}$ or Co in the sample, and the different trends the structural parameters follow when varying them.

Due to the amorphous nature of the Co-W NPs, it is very difficult to fit the EXAFS signal beyond the first coordination shell. Moreover, as it was mentioned above, strong structural disorder in the NPs systems can lead to erroneous interpretation of the EXAFS analysis $[100,101]$. Then, fitting of the Co-W NPs EXAFS signal was performed on the $R$-space in a range from 1.0-3.0 $\AA$ using a hanning window function, so that it covered the Co first coordination shell in the alloy (see Fig. 2.8 and 2.9). For comparison, the EXAFS signal of the bulk $\mathrm{Co}_{3} \mathrm{~W}$ alloy has been fitted in the same $R$ range. An illustration of this Co first coordination shell in the crystal structure of the $\mathrm{Co}_{3} \mathrm{~W}$ alloy is shown in the inset of Fig. 2.8. In this structure, the central Co is surrounded by a total of twelve atoms $\left(N_{\mathrm{T}}=12\right.$, typical in $h c p$ structures $)$, from which eight are other Co atoms $\left(N_{\mathrm{Co}-\mathrm{Co}}=8\right)$ and the remaining four are $\mathrm{W}$ atoms $\left(N_{\mathrm{Co}-\mathrm{W}}=4\right)$. The amplitude reduction factor $\mathrm{S}_{0}^{2}$ and the shift in the threshold energy $\Delta E_{0}$ were set to those obtained for the Co foil fit. A total of 10 fitting parameters were used for an iterative procedure, which

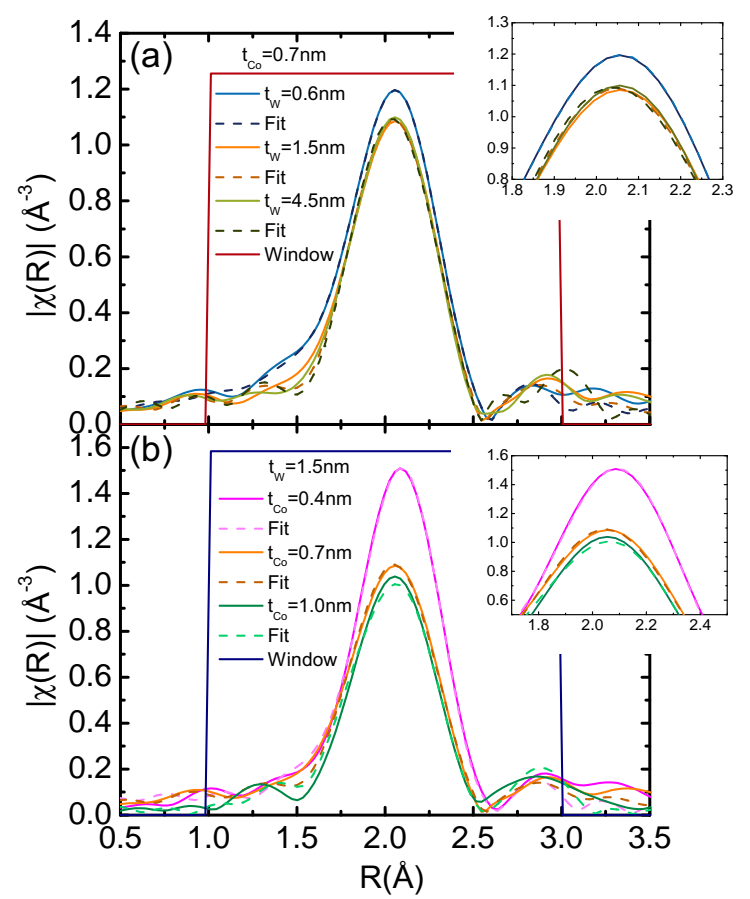

Figure 2.9: Best fits to the first coordination shell on the Co $K$ edge $R$-space EXAFS signal for all five Co-W nanoparticles samples studied. (a) a samples series. (b) $b$ samples series. Inset: zoom of the fitted peak. 
consistently included the fitting of both Co $K$ and $\mathrm{W} L_{3}$ edges (see following section). Results for the $\mathrm{Co}-\mathrm{W}$ NPs and the bulk $\mathrm{Co}_{3} \mathrm{~W}$ fits are shown in Fig. 2.9 and the values obtained in each case are listed in table 2.2. A comparison of the bulk $\mathrm{Co}_{3} \mathrm{~W}$ and one of the Co-W NPs fits is plotted in Fig. 2.8.

A high accordance between the original EXAFS data and their fits is observed for all samples. Fitting results show that the interatomic distance, $R$, and the disorder level quantified by the Debye - Waller factor, $\sigma^{2}$, in the Co-W NPs are strongly affected by the presence of $\mathrm{W}$ in the structure, showing the distortion of the Co-Co and Co-W bonds in the amorphous Co-W alloy. These values found for $\sigma^{2}$ are typical of disordered Co environments, e.g., in Co-rich amorphous alloys $\lfloor 102\rfloor$. It is also observed that the highest $\sigma^{2}$ is obtained in the Co-W bonds, confirming the high amorphicity created by $\mathrm{W}$ in the Co nanoparticles.

The average total coordination of the central Co atom, $N_{\mathrm{T}}$, which includes both Co-Co and Co-W coordination, is reduced from $N=12$ in the $\mathrm{Co}_{3} \mathrm{~W}$ alloy to values as low as $N=9.4$, which corresponds to a decrease of about $22 \%$. This reduction is observed as the amount of $\mathrm{W}$ in the sample increases in the $a$ series and the amount of Co decreases in the $b$ series. However, $N_{\mathrm{T}}$ in most samples still resembles that of the crystal. This is an indication of short range ordering (SRO) in our samples, of chemical or structural origin, or both. Such SRO has been detected in similar $T_{1-x}-M_{x}$ alloys ( $T$ a transition metal and $M$ a metal that favors amorphicity) via EXAFS and Electron Diffraction $\lfloor 92,103\rfloor$. In these alloys, it was found that SRO increases with increasing $T$ content. Indeed, we identify a similar trend of $N_{\mathrm{T}}$ approaching the $N=12$ value in our Co-W NPs as the amount of Co increases in the sample, suggesting a higher $\mathrm{SRO}$ in the sample with $t_{\mathrm{Co}}=1.0 \mathrm{~nm}$.

Variation of the coordination of Co atoms in the Co-W NPs samples with
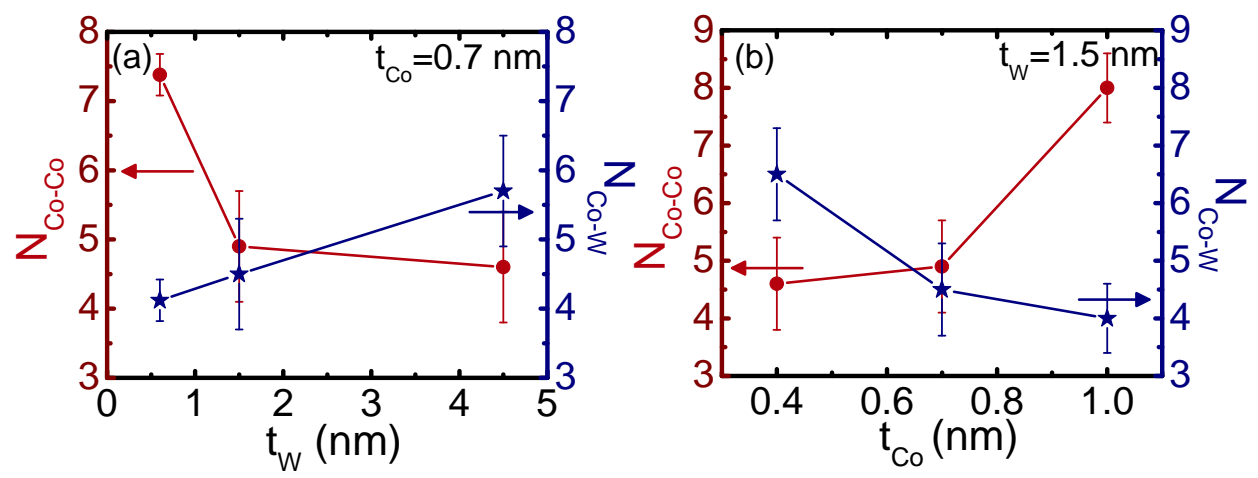

Figure 2.10: Variation of $N_{\text {Co-Co }}$ and $N_{\text {Co-W }}$ obtained from EXAFS fits. (a) a samples series. (b) $b$ samples series. 


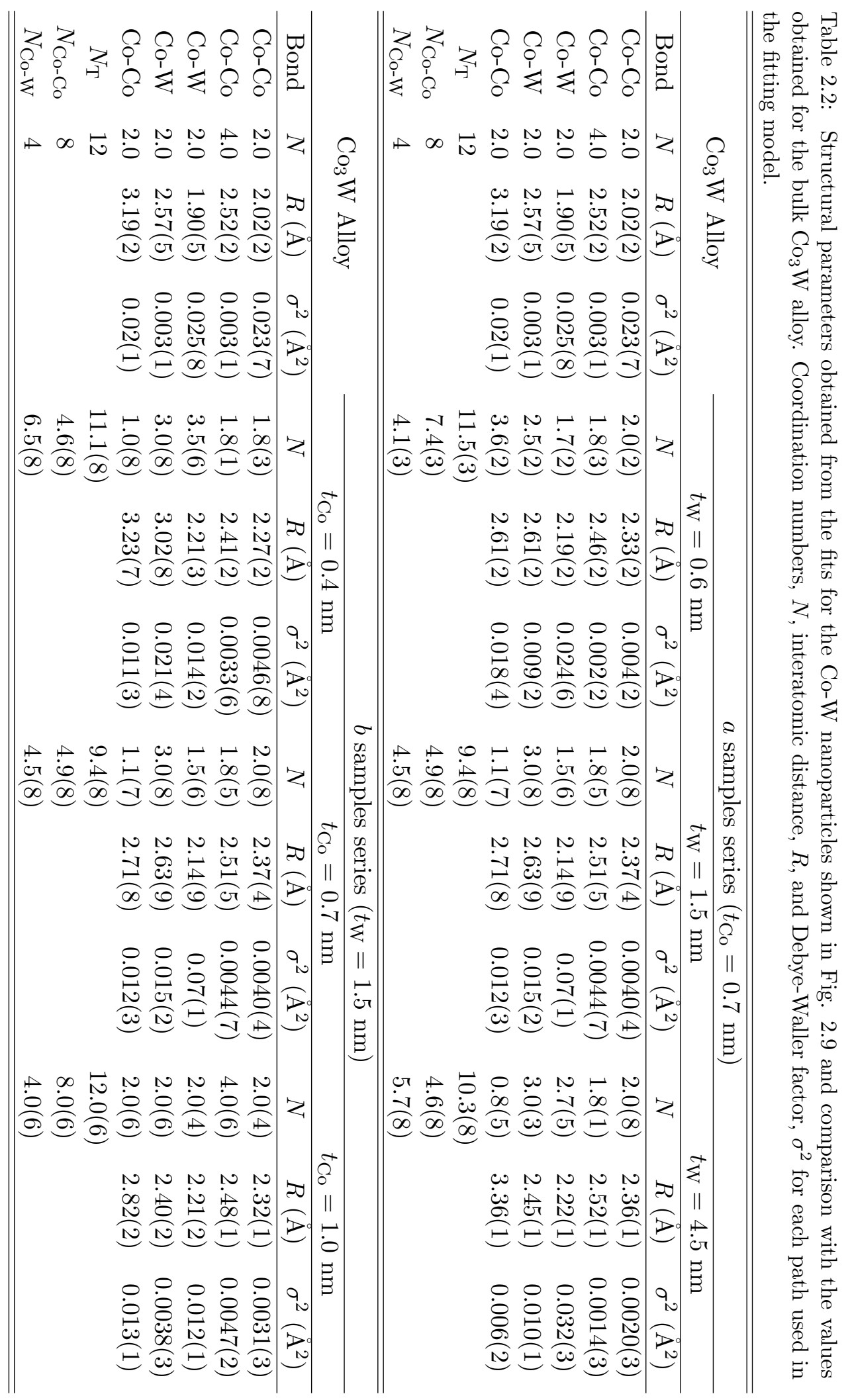


$t_{\mathrm{Co}}$ and $t_{\mathrm{W}}$ is plotted in Fig. 2.10. When analyzing only the coordination between the central Co and their first Co neighbors, $N_{\text {Co-Co }}$ (left y-axis in Fig. $2.10(\mathrm{a})$ and $2.10(\mathrm{~b})$ ), a clear decrease from $N_{\mathrm{Co}-\mathrm{Co}}=8$ in $\mathrm{Co}_{3} \mathrm{~W}$ to values as low as $N_{\mathrm{Co}-\mathrm{Co}}=4.6$ for the smallest particles, is observed. This is a reduction of about $42 \%$ in the Co-Co coordination. In general, it is found that the $N_{\mathrm{Co}-\mathrm{Co}}$ decreases as the amount of Co in the sample decreases in the $b$ samples series, and the as the $\mathrm{W}$ content increases in the $a$ samples series. Similarly, the average coordination of $\mathrm{W}$ atoms surrounding the central Co, $N_{\text {Co-W}}$, increases as the amount of $\mathrm{W}$ in the sample increases, and as the amount of Co decreases, following an almost linear dependence with $t_{\text {Co }}$ and $t_{\mathrm{W}}$ (see right y-axis in Fig. 2.10(a) and 2.10(b)).

A slightly higher coordination has been observed for the sample with the lowest amount of $\mathrm{Co}\left(t_{\mathrm{Co}}=0.4 \mathrm{~nm}\right.$ and $\left.t_{\mathrm{W}}=1.5 \mathrm{~nm}\right)$, which seems to deviate from the trend given the small size of the particles in this sample. This behavior is probably due to the high $x_{\text {para }}$ present in this case $\left(x_{\text {para }}=0.5\right)$ so that the system is less particulate than the others, forming an almost uniformly distributed Co-W amorphous alloy on each layer.

\section{W $L_{3}$ edge results}

Normalized XANES spectra at the $\mathrm{W} L_{3}$ edge for Co-W NPs are shown in Fig. 2.11(a) and Fig. 2.11(b), for $a$ and $b$ sample series, respectively. XANES of $\mathrm{W}$ foil at the same edge is plotted in both cases for comparison. In the case of $\mathrm{W}$, being a $5 d$ metal, $L_{3}$ absorption edge appears for transitions from $2 p$ to both $5 d_{3 / 2}$ and $5 d_{5 / 2}$ bands. Then, an increase in $\mathrm{W} L_{3}$ edge intensity from one sample to the other show an increase in the number of W $5 d$ available empty states. In the $a$ series samples (Fig. 2.11(a)), intensity decreases as $t_{\mathrm{W}}$ increases. For the $b$ series (Fig. 2.11(b)), the white-line is systematically enhanced as the amount of Co increases from zero, in the case of $\mathrm{W}$ foil, to $t_{\mathrm{Co}}=1.0 \mathrm{~nm}$. In both cases, an increase in the $\mathrm{Co} / \mathrm{W}$ ratio gives rise to an increase in the number of W $5 d$ empty states. We may conclude that as this $\mathrm{Co} / \mathrm{W}$ ratio increases there is an electron transfer from $\mathrm{W}$ towards Co.

Fourier transform of the EXAFS data taken at the $\mathrm{W} L_{3}$ edge for the Co-NPs is shown in Fig. 2.12. Fourier transformation was performed on the $\chi(\mathrm{k})$ EXAFS signal on a k-range from 2.3 to $9.8 \AA^{-1}$ using a $\mathrm{k}^{2}$ weight and a sine window function. Fig. 2.12(a) shows results for samples from the $a$ series and Fig. 2.12(b) for the $b$ series, as well as for a $\mathrm{W}$ foil used as a reference and for $\mathrm{Co}_{3} \mathrm{~W}$ alloy for comparison. In both cases it is evident that the $\mathrm{W}$ neighborhood has changed in the Co-W nanoparticles compared to that of metallic $\mathrm{W}$ by the presence of $\mathrm{Co}-\mathrm{W}$ alloying. The first peak in the Co-W 


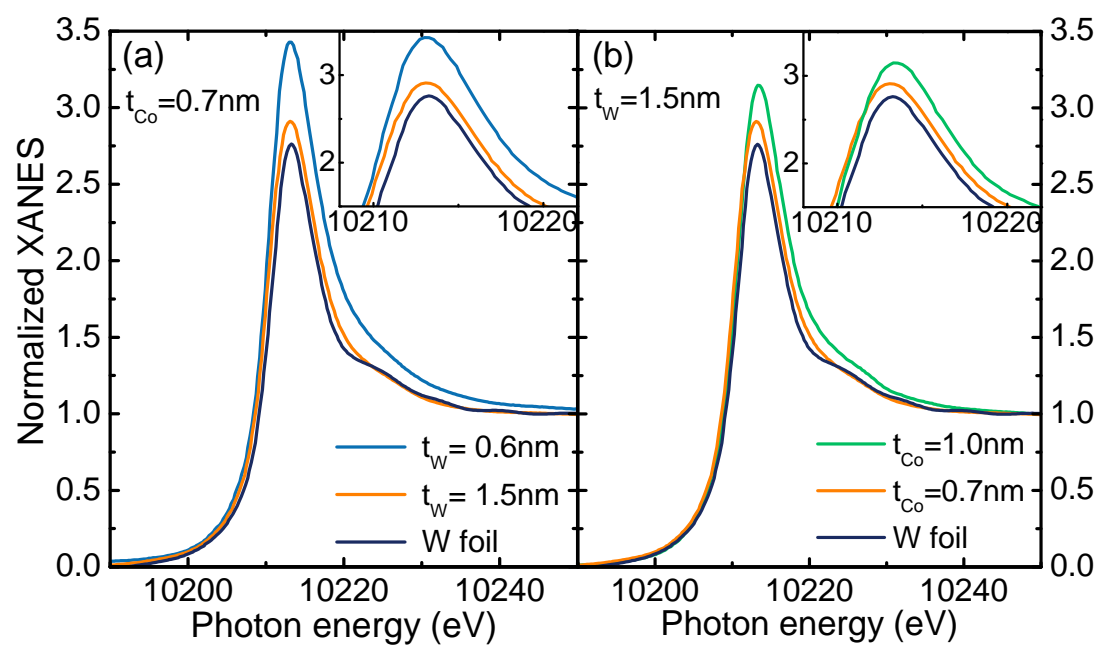

Figure 2.11: XANES spectra at the $\mathrm{W} L_{3}$ edge for samples from the (a) a samples series and (b) $b$ samples series. In both cases, the absorption of the $\mathrm{W}$ foil is shown for reference. Insets show details of the white line intensity.

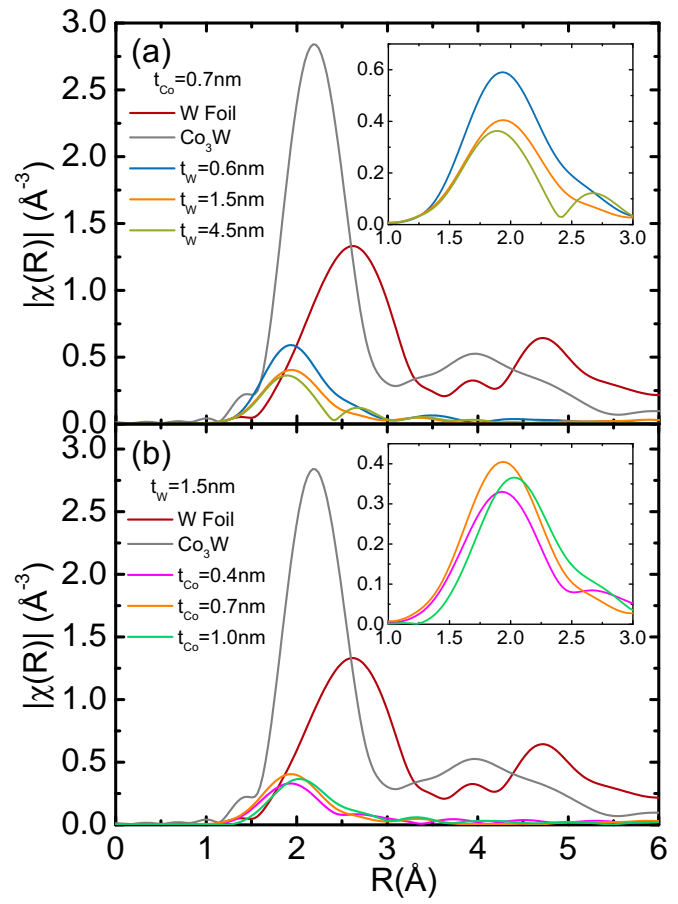

Figure 2.12: Fourier transform of EXAFS signal at the $\mathrm{W} L_{3}$ edge on the Co-W NPs studied. (a) $a$ samples series. (b) $b$ samples series. Measurements for $\mathrm{W}$ foil and $\mathrm{Co}_{3} \mathrm{~W}$ are shown for comparison in both graphs. Insets: zoom of the first coordination shell in the particulate systems. 
samples is very close to that of $\mathrm{Co}-\mathrm{W}$ in the $\mathrm{Co}_{3} \mathrm{~W}$ alloy. For the sample with $t_{\mathrm{W}}=0.6 \mathrm{~nm}$ and $t_{\mathrm{Co}}=0.7 \mathrm{~nm}$ this peak amplitude is higher showing a higher amount of Co neighbors in the $\mathrm{W}$ environment.

EXAFS spectra at the $\mathrm{W} L_{3}$ edge were fitted following a similar procedure to the one for Co $K$ edge, which included an iterative fitting process of both edges. Fitting was performed on the $R$-space in a range that covered the $\mathrm{W}$ first coordination shell (1.5-3.0 $\AA$ ), using a hanning window function (see Fig. 2.13). $\mathrm{S}_{0}^{2}$, and $\Delta E_{0}$ were set to those values obtained for the $\mathrm{W}$ foil signal fit. Different models for fitting were tested: a pure metallic W model, a pure Co-W alloy $\left(\mathrm{Co}_{3} \mathrm{~W}\right)$ and a linear combination of these two phases. The latter was the procedure leading to the best fitting results, shown in Fig. 2.13.

$\mathrm{W}$ environment was found to be consistent with the results of Co-W alloy obtained for the Co $K$ edge, showing evidence of an additional W metal phase in all samples. However, a quantitative determination of this $\mathrm{W}$ metal fraction was prevented given the intrinsic difficulties of fitting EXAFS signals for amorphous systems, as mentioned above $[100,101]$. In spite of it, $\mathrm{W} L_{3}$ EXAFS results show interesting trends as shown in Fig $2.13 \mathrm{a}$, where it is noticeable that the shoulder corresponding to the metallic $\mathrm{W}$ contribution increases as

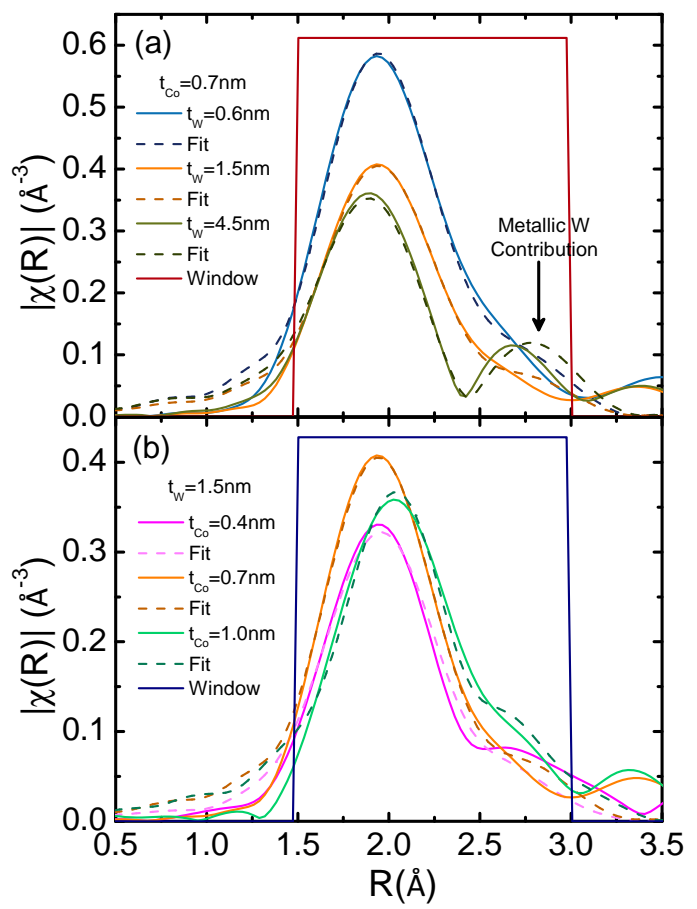

Figure 2.13: Best fits to the first coordination shell on $R$-space EXAFS signal measured at the $\mathrm{W} L_{3}$ edge for the Co-W nanoparticles samples. (a) a samples series. (b) $b$ samples series. 
the amount of $\mathrm{W}$ increases in the sample ( $a$ series). A similar trend was also observed in their fitting results and is well in agreement to what was observed by HRTEM. The fraction of $\mathrm{W}$ metal was found to be around an almost constant value when fitting the samples from the $b$ series (Fig. 2.13(b)).

\subsection{Magnetic study}

\subsubsection{SQUID-based magnetometry}

The magnetic study of both $a$ and $b$ samples series was performed by $\chi_{d c}(T)$, $\chi_{a c}(T)$ and $M(H)$ measurements in a commercial SQUID magnetometer at the Servicio de Medidas Físicas of the Universidad de Zaragoza. $\chi_{d c}(T)$ was measured after zero-field cooling (ZFC) and field cooling (FC) the sample. Temperature was varied between $5 \mathrm{~K}$ and $150 \mathrm{~K}$, and a 200 Oe field was applied. $M(H)$ measurements were performed at $T \approx 3 T_{B}\left(T_{B}\right.$, blocking temperature of the superparamagnetic particles) under applied fields up to 50 kOe. $\chi_{a c}(T)$ was measured by applying an ac field of 4 Oe in amplitude to the sample with frequency $\omega / 2 \pi$ in between $1 \mathrm{~Hz}$ and $480 \mathrm{~Hz}$.

SQUID based magnetic measurements reveal the superparamagnetic behavior of the amorphous Co-W NPs. ZFC and FC curves for both sample series (Fig. 2.14) are similar to those for bare and $\mathrm{Cu}, \mathrm{Ag}$ and $\mathrm{Au}$ - capped Co nanoparticles $\lfloor 11,17,19\rfloor$. At lower temperatures, FC and ZFC for each sample separate and the ZFC curve shows a maximum at a blocking temperature, $T_{B}$. The values of $T_{B}$ for the Co-W NPs are given in table 2.3. $T_{B}$ values in all samples are lower than those found for the bare Co-NPs [17]. $T_{B}$ is also reduced for the $a$ series as the amount of $\mathrm{W}$ in the sample increases for a constant $t_{\mathrm{Co}}$ (Fig. 2.14(a)) and for samples from the $b$ series when the amount of Co decreases at a fixed $t_{\mathrm{W}}$ (Fig. 2.14(b)). At high temperatures, all curves follow the Curie-Weiss law $\chi_{e q}=C /(T-\theta)$. The values of the Curie-Weiss temperature $\theta$ obtained from the curves are shown in table 2.3.

Although $\theta$ values are not negligible, some trends are identified in order to describe the magnetic interactions between Co-W NPs. For the $a$ series samples, $\theta$ remains approximately constant as the amount of $\mathrm{W}$ increases, suggesting that interparticle interactions are quite similar in the series and decrease on a small scale when adding $\mathrm{W}$. This is understandable since particles are similarly and uniformly distributed on the layers of these three samples, as demonstrated by HRTEM (see analysis of $N_{S}$ in section 2.2.1). Instead, for the $b$ series, $\theta$ values have a drastic change, increasing in one order of magniture from sample with $t_{\mathrm{Co}}=0.4 \mathrm{~nm}$ to the one with $t_{\mathrm{Co}}=1.0 \mathrm{~nm}$. Thus, the magnetic interactions in the Co-W NPs increase as the amount 


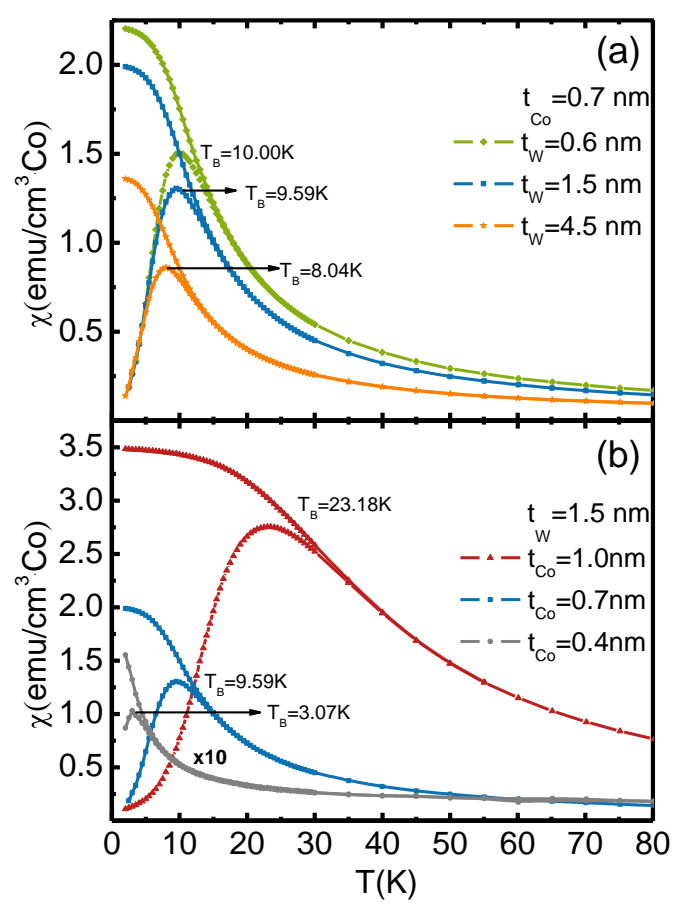

Figure 2.14: DC susceptibility curves for samples from the $a$ series in (a) and from the $b$ series in (b).

of Co increases because particles are bigger and closer. These results of the magnetic interactions in the Co-W NPs are well supported by analysis of the variation of the $\mathrm{FC} / \mathrm{ZFC}$ - curves height ratios $\lfloor 104\rfloor$, which show the same trend. This is, FC/ZFC - curves height ratios for the $a$ series are almost equal and they decrease as $t_{\mathrm{Co}}$ increases for the $b$ series samples.

Magnetization as a function of field in the paramagnetic region $(M(H)$ at $\left.T \approx 3 T_{B}\right)$ for both sample series are plotted in Fig. 2.15. These $M(H)$ curves were identical when measured with the field parallel and perpendicular to the sample plate, therefore the particle anisotropy axes are randomly distributed in all samples and the demagnetization factor plays no role in the present case.

Values of the magnetization per Co atom deposited in the samples, $m_{\text {tot }}$, obtained from saturation are listed in table 2.3 and plotted in Fig. 2.16. It was found that $m_{\text {tot }}$ decreases for samples from $a$ series as the $\mathrm{W}$ capping layer thickness increases for a constant $t_{\mathrm{Co}}$ (Fig. 2.15(a) and 2.16(a)) and in samples from the $b$ series as the amount of Co decreases for a fixed $t_{\mathrm{W}}$ (Fig. 2.15(b) and 2.16(b)). The magnetization is supported by the Co atoms since $\mathrm{W}$ has a negligible induced moment. ${ }^{9}$ Then, there is a strong reduction on the magnetic

\footnotetext{
${ }^{9}$ See details of the induced magnetic moments in the $\mathrm{W}$ atoms of these samples in chapter
} 3. 

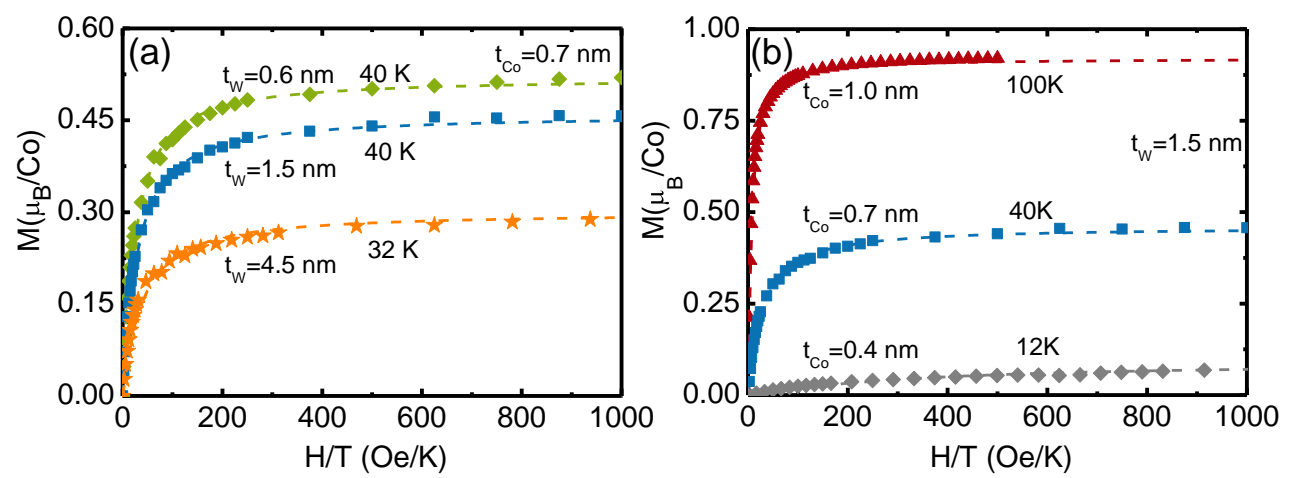

Figure 2.15: Magnetization curves for samples for samples from the $a$ series in (a) and from the $b$ series in (b). The dashed lines are fitted Langevin curves with $\left\langle D_{\text {mag }}\right\rangle$ and $\sigma$ parameters given in table 2.3 .

moment of the Co atoms due to the formation of Co-W amorphous alloy. This reduction may be due to combined effect of charge transfer, hybridization and environmental effects, as it is described in Ref. [27]. According to this author, when the number of Co first neighbors is higher than 3, Co atoms carry their full magnetic moment of $1.7 \mu_{B} / \mathrm{Co}[27]$. This is the case of previously studied bare Co nanoparticles [19]. However, if the Co atom has less Co atoms as first neighbors, e.g. 2 or 3 Co atoms, its magnetic moment decreases to about $0.5 \mu_{B} /$ Co. The latter value is well in agreement with the moments found for Co atoms in the samples studied in this work. The strong reduction of the Co moment in our samples is due to a decrease in the Co coordination of the Co atoms because of the presence of $\mathrm{W}$, demonstrated via EXAFS analysis in the previous section. Such decrease in $T_{1-x}-M_{x}$ alloys ( $T$ being a transition metal and $M$ usually a non-magnetic metal) has been reported in previous works $\lfloor 105\rfloor$ and will be further discussed in section 2.4.

For those samples where the number of particles per unit surface, $N_{S}$, was identified from the STEM images in the morphology characterization, we can easily determine the magnetic moment per particle, $m_{\text {Part }}$. This is obtained by dividing the saturation magnetization by $N_{S}$, both, per unit surface. $m_{\text {Part }}$ values are listed in table 2.3. The average amount of Co atoms per particle is also easily determined by the ratio of deposited Co and number of particles, per unit surface. The resulting moment per Co atom (table 2.3) decreases with increasing the amount of $\mathrm{W}$ in the $a$ series samples from 0.52 to 0.30 $\mu_{B} / \mathrm{Co}$, for $t_{\mathrm{W}}=0.6$ and $4.5 \mathrm{~nm}$, respectively. Also, it strongly decreases with reduction of the amount of $\mathrm{Co}$ in the $b$ series samples from 0.93 to $0.08 \mu_{B} / \mathrm{Co}$ for $t_{\mathrm{Co}}=1.0$ and $0.4 \mathrm{~nm}$, respectively. Thus, the reduction in Co moment expected for Co-W alloying $[25,27\rfloor$ is properly verified. 


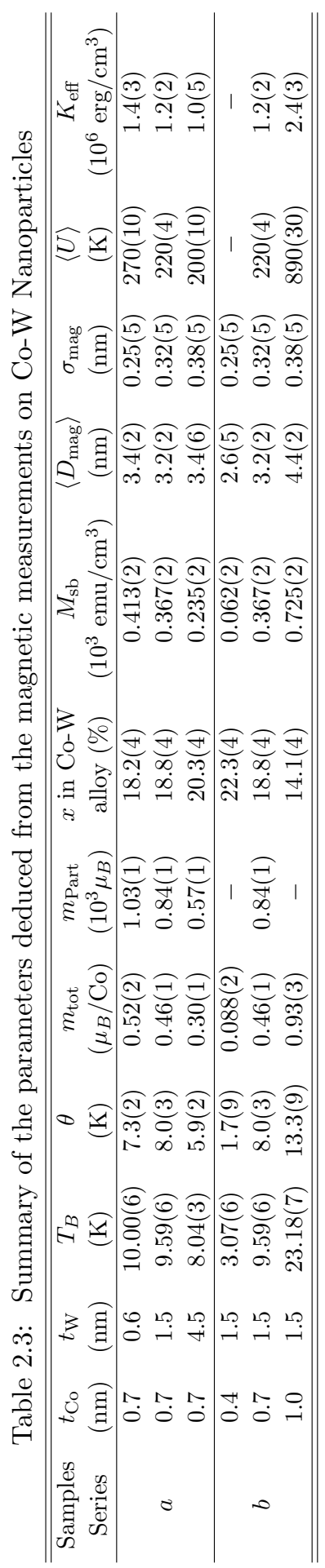



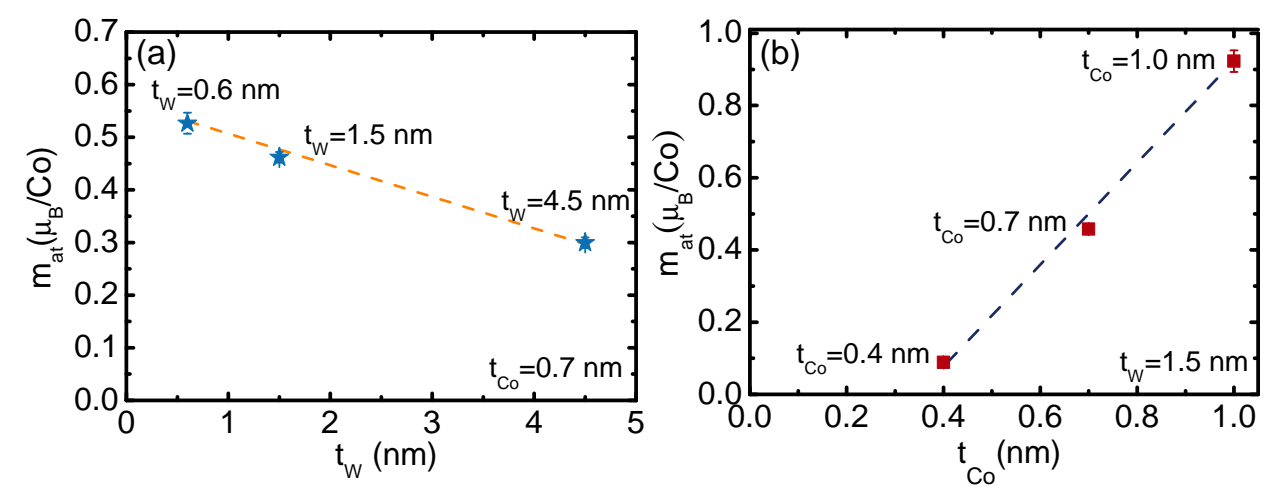

Figure 2.16: Total Co magnetic moment per Co atom in the Co-W NPs as deduced from the $M(H)$ measurements. (dashed lines) Guide to the eye. (a) a samples series. (b) b samples series.

An average $\mathrm{Co}_{1-x} \mathrm{~W}_{x}$ alloy composition in the Co- $\mathrm{W}$ NPs may be proposed by comparing the $m_{\text {tot }}$ values to those measured for Co-W films deposited by rf sputtering in Ref. [26]. Values of $\% \mathrm{~W}$ in the alloy are shown in table 2.3. The $\mathrm{W}$ concentration that explains the reduction in magnetic moment ranges from 18.2 to 20.3 at \% in the $a$ samples series, and from 14.1 to 22.3 at $\%$ in the $b$ samples series.

From the equilibrium magnetic properties of each sample we can determine the particle size distribution and compare it with the results obtained by HRTEM and STEM. This is obtained by fitting the $M(H)$ curves at high T to a Langevin function, averaged over the appropriate particle size distribution. We define $g\left(D_{\mathrm{mag}}\right)$ as the gaussian distribution of a number of particles having a diameter equal to $D_{\text {mag }}$. For spherical particles, the particle magnetization is written as $m_{\text {part }}=\pi M_{\mathrm{sb}} D_{\mathrm{mag}}^{3} / 6$, where $M_{\mathrm{sb}}$ is defined as the particle magnetic density, calculated from the saturation magnetization values and the density of the Co-W alloy formed on each sample as

$$
M_{\mathrm{sb}}(x)=\frac{m_{\mathrm{tot}}\left(\mu_{B} / C o\right) \rho(x)}{M_{\mathrm{w}}(x)} N_{a} \mu_{B}
$$

where $x$ gives the $\mathrm{Co}_{1-x} \mathrm{~W}_{x}$ alloy composition, $\rho(x)$ is the density and $M_{\mathrm{w}}(x)$ the molecular weight of the alloy, $N_{a}$ is the Avogadro number and $\mu_{B}$ the Bohr magneton. Since there are no direct values in the literature for the densities of the Co-W alloys found for our samples, we need to calculate them. Knowing that the density for metallic $\mathrm{Co}$ is $8.9 \mathrm{~g} / \mathrm{cm}^{3}$ and the one for $\mathrm{Co}_{3} \mathrm{~W}$ alloy is $12.7 \mathrm{~g} / \mathrm{cm}^{3}\lfloor 106\rfloor$, we can apply linear approximation for $\rho(x)$ vs. $x$. Given the $x$ values found for our Co-W samples (listed in table 2.3) we can interpolate each composition value and get its respective density. 
The experimental data are fitted by the expression:

$$
\begin{aligned}
& M(H, T)=x_{\text {para }} m_{\text {para }} \\
& +\left(1-x_{\text {para }}\right) M_{s b} \frac{\int g\left(D_{\mathrm{mag}}\right) V\left[M(H, T, \mu) / m_{\text {tot }}\right] d D}{\int g\left(D_{\mathrm{mag}}\right) V d D_{\mathrm{mag}}}
\end{aligned}
$$

Let us recall that $x_{\text {para }}$ gives the fraction of non-aggregated Co atoms present in the samples. In the case of bare and noble-metal capped Co NPs they give a paramagnetic contribution as a positive slope of $M(H)$ at high fields $\lfloor 17,19\rfloor$. In our Co-W alloy NPs, the non-aggregated Co atoms are also alloyed with W. They do not contribute to the magnetic signal, i.e., at high fields the slope of $M(H)$ is negligible. Then $m_{\text {para }}$ in equation 2.2 is null and equation 2.2 is reduced to the second term.

Results for these fits are the dashed lines in Fig. 2.15. The average magnetic diameter values $\left\langle D_{\mathrm{mag}}\right\rangle$ and the distribution width for the Gaussian distribution of particle size, $\sigma_{\text {mag }}$ obtained are listed in table 2.3. These parameters were confirmed by fitting the equilibrium susceptibility $1 / \chi_{\text {eq }}(T)$ in the superparamagnetic temperature regime, measured for the same samples. This is, for a system of particles of that diameter and size distribution, $\chi_{\text {eq }}$ can be expressed as

$$
\chi_{\mathrm{eq}}=\frac{\int g\left(D_{\mathrm{mag}}\right) V\left(M_{\mathrm{sb}}^{2} V / 3 k_{B} T\right) d D_{\mathrm{mag}}}{\int g\left(D_{\mathrm{mag}}\right) V d D_{\mathrm{mag}}}
$$

Comparing the values of $\left\langle D_{\text {eff }}\right\rangle$ obtained from the morphological analysis described in section 2.2.1 with the $\left\langle D_{\mathrm{mag}}\right\rangle$ derived from this magnetic analysis, we note that the latter is smaller. From the above facts we may conclude that our amorphous Co-W NPs are composed by a magnetic $\mathrm{Co}-\mathrm{W}$ alloy core surrounded by unalloyed metallic $\mathrm{W}$, which tends to fill the interparticle space, as described in sections 2.2.1 and 2.2.2.

\subsubsection{Magnetic anisotropy of the Co- $\mathrm{W}$ particles}

The phenomenon of superparamagnetic blocking described in the FC-ZFC curves analysis is related to the magnetic anisotropy of the particles. The anisotropy favors some particular orientations of the magnetic moment, two opposite to each other in the simplest case of uniaxial anisotropy, which are separated by activation energy barriers $U$. The rate at which the magnetic moment of the particles flips from one orientation to the other is determined by this anisotropy $K_{\text {eff }}$, so that we write $U=K_{\text {eff }} V$, with $V$ being the particle 
magnetic volume. The relaxation time, $\tau$, follows an Arrhenius' law $(\tau=$ $\tau_{0} \exp \left(U / k_{B} T\right)$ ), where $\tau_{0} \approx 10^{-10}-10^{-13}$ s is the inverse attempt frequency that depends on the damping of the magnetic moments by the phonons. The superparamagnetic blocking occurs when $\tau=t_{e}$, where $t_{e}$ is the measuring time of each experimental point, so $T_{B} \cong \alpha U / k_{B} \ln \left(t_{e} / \tau_{0}\right)$ where $\alpha$ is a constant that depends on the width of the particle size distribution.

An accurate way to obtain $U$ is derived from the full distribution of activation energies $f(U)$ and then to find which value of $U$ corresponds to particles with a diameter equals to $D_{\text {mag. }}$. A detailed description of this procedure can be found in Ref. [17]. $f(U)$ can be directly determined from the analysis of the imaginary part of $\chi_{\mathrm{ac}}(\mathrm{T})$, measured near the superparamagnetic blocking temperature $T_{B}$. For non-interacting particles we write

$$
\chi^{\prime \prime} \simeq \frac{\pi}{2} k_{B} T \chi_{\mathrm{eq}}(T, U) f\left(U_{b}\right)
$$

where we define $U_{b}=k_{B} T \ln \left(1 / \omega \tau_{0}\right)$, as a scaling variable, corresponding to the activation energy of those particles having $\tau=t_{e}=1 / \omega$ at a given $T$. The frequency dependent $\chi_{\text {ac }}$ should converge into a single curve when plotting $\chi^{\prime \prime}$ vs. $U_{b} / k_{B} . f(U)$ can be determined from equation 2.4 from a $\chi^{\prime \prime}\left(U_{b}\right)$ fit. In Fig. 2.17 we have plotted the result obtained from this $\chi_{\mathrm{ac}}(\mathrm{T})$ analysis, including the $\chi^{\prime \prime}\left(U_{b}\right)$ fit, for the sample with $t_{\mathrm{Co}}=0.7 \mathrm{~nm}$ and $t_{\mathrm{W}}=1.5$ $\mathrm{nm}$. We also show the Arrhenius plot of $\ln (1 / \omega)$ vs. $1 / T_{B}$, from the analysis

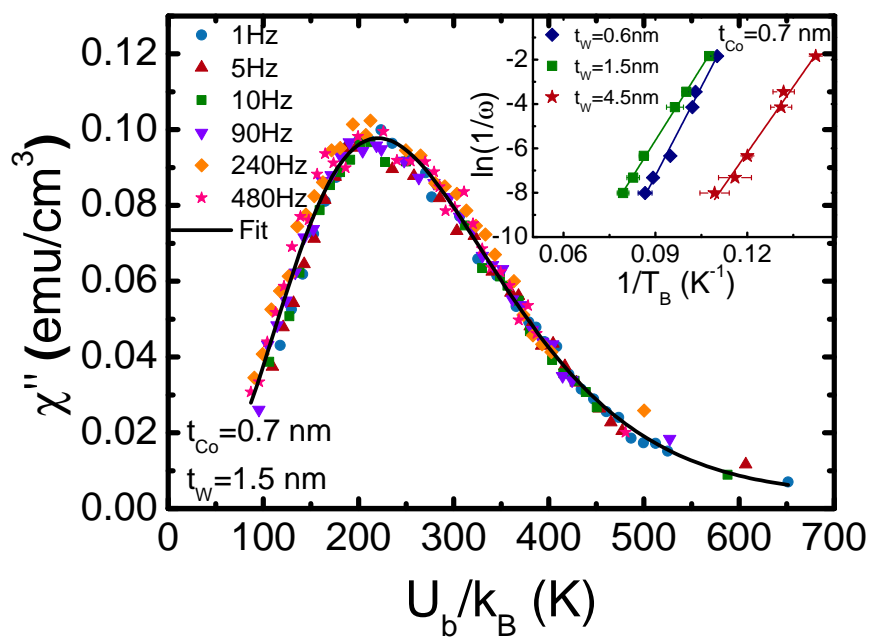

Figure 2.17: Imaginary part of the ac susceptibility for the sample with $t_{\mathrm{Co}}=0.7 \mathrm{~nm}$ and $t_{\mathrm{W}}=1.5 \mathrm{~nm}$ plotted as a function of the scaling variable $U_{b}$. The solid line is the fitting result. Inset: Arrhenius plot of the measuring time $(1 / \omega)$ as function of $T_{B}$, as obtained from $\chi^{\prime \prime}$ measurements for the $a$ series samples. 
obtained from $\chi^{\prime \prime}$ measurements for the $a$ series samples. In the case of the sample with the lowest amount of $\mathrm{Co}\left(t_{\mathrm{Co}}=0.4 \mathrm{~nm}\right.$ and $\left.t_{\mathrm{W}}=1.5 \mathrm{~nm}\right)$ this analysis was not performed since the $\chi_{a c}$ signal was negligible.

$K_{\text {eff }}$ is then obtained from $U\left(\left\langle D_{\text {mag }}\right\rangle\right)=K_{\text {eff }}(\pi / 6)\left\langle D_{\text {mag }}\right\rangle^{3}$, where $\left\langle D_{\text {mag }}\right\rangle$ denotes the average magnetic diameter of the spherical particles. All this analysis is performed assuming that the magnetic moment is just supported by the Co-W magnetic alloy core. The values of the average $\langle U\rangle$ and $K_{\text {eff }}$ obtained for all samples are shown in table 2.3 .

$K_{\text {eff }}$ in our Co-W NPs decreases with increasing $t_{\mathrm{W}}$ for the $a$ series samples and with decreasing $t_{\mathrm{Co}}$ for the $b$ series samples. This gives $K_{\text {eff }}$ a trend directly proportional to $\left\langle D_{\mathrm{mag}}\right\rangle$, suggesting that surface anisotropy is not playing an important role in the $K_{\text {eff }}$ as in other particulate systems $[11,19,78-81\rfloor$. Further discussion on these results will be presented in section 2.4 .

\subsubsection{X-ray Magnetic Circular Dichroism magnetometry}

The XAS and XMCD experiments at the Co $L_{3}(778.1 \mathrm{eV})$ and Co $L_{2}(793.2$ $\mathrm{eV}$ ) edges were performed at the ID08 beamline of the ESRF. An APPLE-II undulator and a spherical grating monochromator were used. The degree of polarization was $\sim 100 \%$. The total electron yield (TEY) detection method was employed. The beam direction and parallel magnetic field applied had an incident angle of $10-15^{\circ}$ with respect to the normal to the plate sample. The XMCD signal was obtained by a direct difference of the XAS spectra recorded with opposite helicities at each magnetic field value and for both orientations of the field. The applied field was $20 \mathrm{kOe}$ and the temperature was $T=7 \mathrm{~K}$ in all cases.

$\mathrm{XMCD}$ is a very powerful technique that allows to perform an element selective magnetometry on our Co-W NPs. From a XMCD experiment at the $L_{2,3}$ edges of Co one obtains, through the sum rules $[64,65$. described in section 1.4.2, the spin and orbital moments averaged over the volume of the particle, and on the number of holes $n_{h}$ in the empty exchange split $\mathrm{n} d$ sub-bands (Co $3 d$ in the present case). The sum rules have been applied to the spectra to derive the orbital, spin and total magnetic moments per $3 d$ hole of Co, $m_{\mathrm{L}} / n_{h}$, $m_{\mathrm{S}} / n_{h}$ and $m_{\text {at }} / n_{h}$, respectively, with the symbols and sign criteria defined in Ref. $[66\rfloor$. We define the constant $C=\left(I_{L 2}+I_{L 3}\right)$, where $I_{L 2}$ and $I_{L 3}$ are the white-line integrated intensities after subtraction of the contributions from transitions to the continuum. It is noteworthy that in the present case of amorphous particles, with their individual anisotropy axes oriented at random, the dipolar term $m_{D}$ in $m_{\text {Seff }}=m_{\mathrm{S}}+m_{\mathrm{D}}$ cancels out, so that the sum rule $(A-2 B)=-C m_{\mathrm{S}} /\left(n_{h} \mu_{B}\right)$ yields the spin component $m_{\mathrm{S}} / n_{h} . A$ and $B$ are the 
integrated areas of the $L_{3}$ and $L_{2}$ XMCD peaks, respectively. Besides, from the ratio of the sum rules $m_{\mathrm{L}} / m_{\mathrm{S}}$ is directly derived, which is independent of $n_{h}$ and of the normalization method used.

XMCD also allows us to study the changes in the orbital magnetic moment in the Co-W NPs samples as a function of $t_{\mathrm{W}}$ and $t_{\mathrm{Co}}$ by obtaining the average $m_{\mathrm{L}} / n_{h}$ per Co atom present in the sample. In the experiments a magnetic field of $20 \mathrm{kOe}$ is applied, sufficient to saturate the particle spin magnetization. The contribution of the orbital moment to the XMCD signal is the averaged projection of the local orbital magnetic moments in the direction of the applied field (parallel to the photon beam direction in the experiments).

The experimental XMCD spectra at the Co $L_{2,3}$ edges for sample with $t_{\mathrm{Co}}=0.7 \mathrm{~nm}$ and $t_{\mathrm{W}}=1.5 \mathrm{~nm}$ is depicted in Fig. 2.18, together with its XAS, for normalization sake. All XMCD results are qualitatively similar,

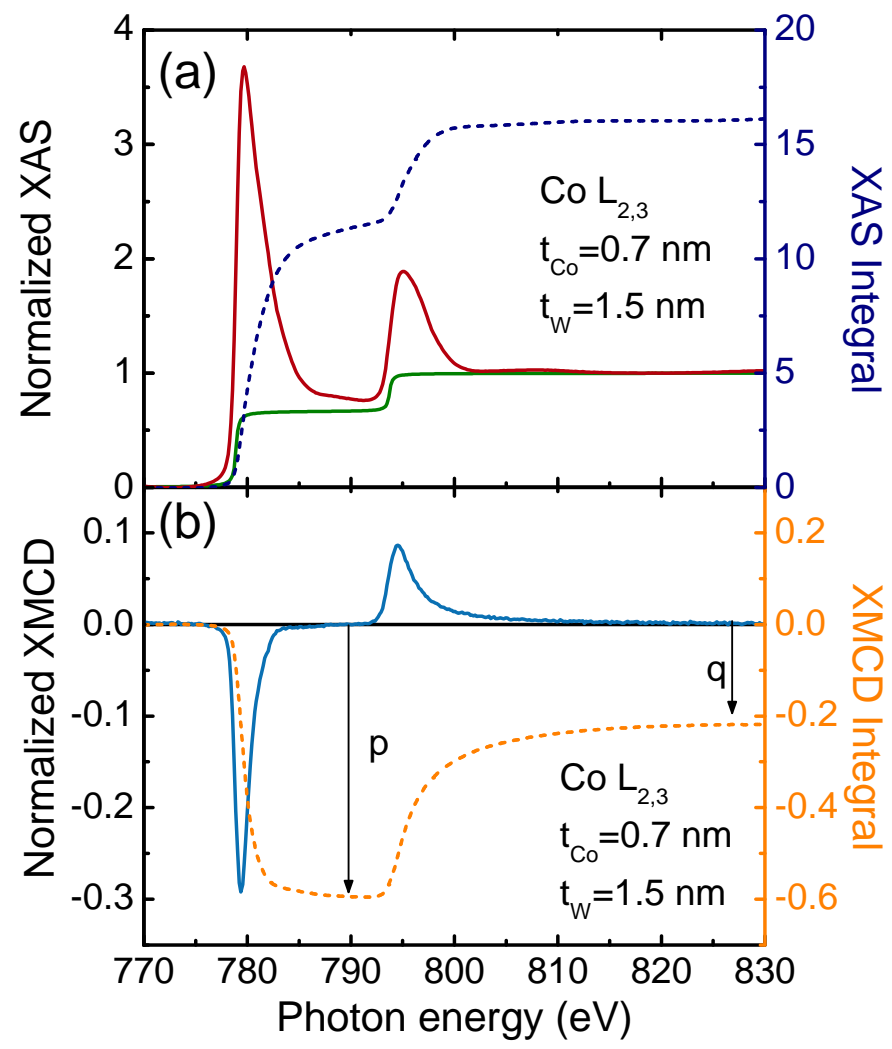

Figure 2.18: Co- $L_{2,3}$ edges spectra of the $t_{\mathrm{Co}}=0.7 \mathrm{~nm}, t_{\mathrm{W}}=1.5 \mathrm{~nm}$ sample measured at $\mathrm{T}=5 \mathrm{~K}, H=20 \mathrm{kOe}$ and incident angle $10^{\circ}$. (a) (red) Normalized XAS spectra mean curve. (green) The double-step function used is also indicated. (dark blue) Integrated white line. (b) (light blue) Normalized XMCD spectra, (orange) integrated area. 
though the actual derived $A$ and $B$ values differ. The derived $m_{\mathrm{L}} / m_{\mathrm{S}}, m_{\mathrm{L}} / n_{h}$ and $m_{\mathrm{S}} / n_{h}$ values are given in table 2.4 , along with the values of the total moment $m_{\text {tot }} / n_{h}$.

Self-saturation effects may affect these TEY measurements in principle. It is customarily corrected in thin films [109], and has been done in the case of rather large Co and Fe nanoparticles $[110,111]$. To correct for this effect the electron escape depth parameter $\lambda_{e}$ at low kinetic energies of the particular material is needed. The Co-W alloys $\lambda_{e}$ at low energies is not known, but at higher energies, of the order of $100 \mathrm{eV}$, it is very near to that for Co. ${ }^{10}$ Under this hypothesis, the correction due to saturation effects would be very similar to that needed for Co particles of the same size. It has been estimated that for Co particles with diameter $D=3 \mathrm{~nm}$ the $m_{\mathrm{S}}$ and $m_{\mathrm{L}}$, parameters obtained with the sum rules from uncorrected XAS and XMCD spectra, may be underestimated by 2 and $13 \%$, respectively [110], which amounts to an underestimation of $3 \%$ in $m_{\text {tot }}$. Such a difference is comparable to the other sources of error in these experiments, like the presence of $x_{\text {para }}$, or the size distribution of the particles. Therefore, to account for this effect, we have added this source of error to the total estimated errors in the table 2.4 data, and should take conclusions from the trends rather than from the absolute values.

The variation of the $m_{\mathrm{L}} / m_{\mathrm{S}}$ ratio as a function of the amount of Co and $\mathrm{W}$ in the samples can be analyzed from the values listed in table 2.4. In $b$ samples series, for $t_{\mathrm{Co}}=0.4 \mathrm{~nm}$ the ratio is much larger than the value for bulk Co $\left(m_{\mathrm{L}} / m_{\mathrm{S}}\right.$ Co Bulk $\left.=0.078\right)$, and decreases towards that value as the $t_{\mathrm{Co}} / t_{\mathrm{W}}$ ratio increases, being quite close for $t_{\mathrm{Co}}=1.0 \mathrm{~nm}\left(m_{\mathrm{L}} / m_{\mathrm{S}}=0.071\right)$ (see

Table 2.4: $m_{\text {tot }} / n_{h}, m_{\mathrm{L}} / n_{h}, m_{\mathrm{S}} / n_{h}$ and $m_{\mathrm{L}} / m_{\mathrm{S}}$ per Co atom as determined from XMCD measurements. For the $f c c$ bulk Co, $m_{\mathrm{L}} / m_{\mathrm{S}}=0.078$ 107, $m_{\mathrm{L}} / n_{h}=0.134$ and $m_{\mathrm{S}} / n_{h}=0.248 \mu_{B}$ per $3 d$ hole $\left[67 .\left(3 d n_{h}^{\text {Co }}=2.49\right)\lfloor 108\rfloor\right.$.

\begin{tabular}{ccccccc}
\hline $\begin{array}{c}\text { Samples } \\
\text { series }\end{array}$ & $\begin{array}{c}t_{\mathrm{Co}} \\
(\mathrm{nm})\end{array}$ & $\begin{array}{c}t_{\mathrm{W}} \\
(\mathrm{nm})\end{array}$ & $m_{\mathrm{L}} / m_{\mathrm{S}}$ & $\begin{array}{c}m_{\mathrm{L}} / n_{h} \\
\left(\mu_{B}\right)\end{array}$ & $\begin{array}{c}m_{\mathrm{S}} / n_{h} \\
\left(\mu_{B}\right)\end{array}$ & $\begin{array}{c}m_{\text {tot }} / n_{h} \\
\left(\mu_{B}\right)\end{array}$ \\
\hline \multirow{3}{*}{$a$} & 0.7 & 0.6 & $0.082(1)$ & $0.018(1)$ & $0.216(3)$ & $0.234(3)$ \\
& 0.7 & 1.5 & $0.085(1)$ & $0.015(1)$ & $0.172(4)$ & $0.186(4)$ \\
& 0.7 & 4.5 & $0.079(2)$ & $0.010(1)$ & $0.120(5)$ & $0.130(6)$ \\
\hline \multirow{3}{*}{$b$} & 0.4 & 1.5 & $0.159(7)$ & $0.007(1)$ & $0.042(4)$ & $0.048(4)$ \\
& 0.7 & 1.5 & $0.085(1)$ & $0.015(1)$ & $0.172(4)$ & $0.186(4)$ \\
& 1.0 & 1.5 & $0.071(1)$ & $0.021(1)$ & $0.290(9)$ & $0.310(9)$ \\
\hline \hline
\end{tabular}

\footnotetext{
${ }^{10}$ Data obtained from the NIST Electron Effective-Attenuation-Length Database, http: //www.nist.gov/srd/nist82.cfm
} 

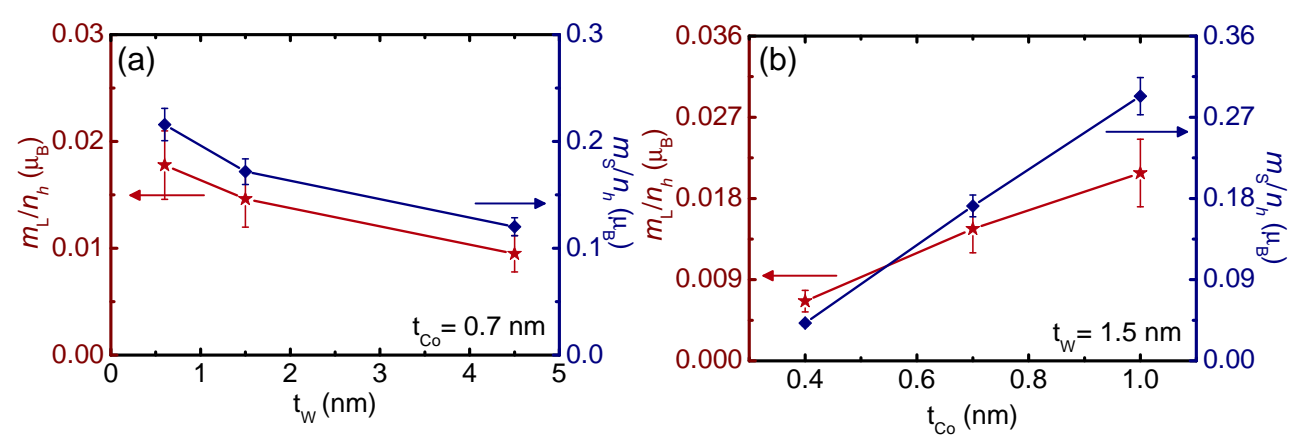

Figure 2.19: Dependence of Co $m_{\mathrm{L}} / n_{h}$ and $m_{\mathrm{S}} / n_{h}$, on the Co and $\mathrm{W}$ composition. (a) $a$ samples series, (b) $b$ samples series.

table 2.4 and Fig. 2.19(b)). The reason for this difference at low $t_{\mathrm{Co}} / t_{\mathrm{W}}$ can be found in the unusually low value of $m_{\mathrm{S}} / n_{h}\left(0.042 \mu_{B}\right.$ per $3 d$ hole $)$ for $t_{\mathrm{Co}}=0.4 \mathrm{~nm}$. Although the $m_{\mathrm{L}} / n_{h}$ decreases with $\mathrm{W}$ alloying, the reduction of $m_{\mathrm{S}} / n_{h}$ is much stronger (see Fig. 2.19(b)). Therefore, alloying with W affects essentially the difference between up and down filled states in the Co $3 d$ band. In contrast, the $m_{\mathrm{L}} / m_{\mathrm{S}}$ ratio as a function of the $\mathrm{W}$ capping thickness is practically constant (see variation of $m_{\mathrm{L}} / n_{h}$ and $m_{\mathrm{S}} / n_{h}$ in Fig. 2.19(a)), i.e., the compositional $\mathrm{Co} / \mathrm{W}$ ratio in the alloys obtained in $a$ samples series is similar (see also alloys composition in table 2.3). It may be concluded that the crucial factor to reach the different alloys in the Co-W NPs depends more on the amount of Co available for alloying than on the amount of $\mathrm{W}$ in the sample.

We may estimate $n_{h}$ for each Co-W composition by substituting in the $m_{\text {tot }} / n_{h}$ values given in table 2.4 , those $m_{\text {tot }}$ determined from $M(H)$ measurements in the previous section (see table 2.3). Although W may be polarized by Co, its contribution to the net magnetization is orders of magnitude lower, ${ }^{11}$ so it is considered as negligible in this analysis. Therefore, the magnetization of our samples is mainly due to the Co present in the Co- $\mathrm{W}$ particles, so we may assume that both SQUID and XMCD magnetometry techniques yield the average total Co moment at saturation (for the sake of comparison we have used values at $H=20 \mathrm{kOe}$ ). Thus, from the comparison of both values, we can extract the variation of the $n_{h}$ in the Co $3 d$ band in the Co-W NPs. For the $a$ series samples, $n_{h}$ was found to be around an almost constant value of $n_{h}=2.4(1)$. Within experimental errors, this result is on the order or slightly lower than that for bulk Co $\left(n_{h}=2.49\right)$ [108」, and is well in agreement with

\footnotetext{
${ }^{11}$ XMCD measurements at the $\mathrm{W} L_{2,3}$ edges on these W-capped Co nanoparticles samples have been performed to determine the induced magnetic moment of $\mathrm{W}$. It was found to be on the order of $\sim 10^{-3} \mu_{B} / \mathrm{W}$ at $10 \mathrm{~K}$, which is two orders of magnitude lower than the Co moment measured in the samples. These results will be described in detail in chapter 3
} 
the insignificant variation observed in the pre-edge peak of Co $K$ XAS spectra for the same samples, described in section 2.2 .2 ; i.e., the Co $3 d-\mathrm{W} 5 d$ hybridization is hardly affected by small variations of the amount of $\mathrm{W}$ for a fixed Co, showing once again that the alloy composition the three samples along this series is similar. For the $b$ series samples, $n_{h}$ was found to vary within a $20 \%$ of the average value found for the $a$ series. The general trend was that of increase as the amount of Co in the sample increases for a fixed $t_{\mathrm{W}}$ value. This latter result is also in agreement to what was observed at the Co $K$ edge XAS measurements in this samples series since it gives evidence of increasing localization of the $3 d$ states in $\mathrm{Co}$ as the $\mathrm{Co} / \mathrm{W}$ ratio in the sample increases.

\subsection{Discussion}

We have found that the magnetic moment per Co atom in our alloy decreases with increasing $\mathrm{W}$ content. Such dependence has been extensively reported both in crystalline and amorphous alloys following the formula $T_{1-x}-M_{x}$, with $T$ usually being $\mathrm{Fe}$, $\mathrm{Co}$ or $\mathrm{Ni}$, and $M$ an early transition metal that favors the amorphicity in the alloy $\lfloor 105\rfloor$. A generalized Slater-Pauling model based on the existence of a gap, or minimum, in the conduction-band density of states has been used to describe this trend. [105] Such gap tends to conserve the number of conduction electrons in an alloy series leading to a linear variation of $m_{\text {tot }}$ (the atom-averaged magnetic moment of the alloy) with $Z_{M}$ (the atomic valence of the $M$ metal, $Z_{M}=6$ for $\left.\mathrm{W}\right)\lfloor 105\rfloor$. The relation of $m_{\text {tot }}$ as a function of $x$ is then expressed as

$$
m_{\text {tot }}(x)=m_{\text {metal }}-x\left(10+Z_{M}-Z_{T}\right) \mu_{\mathrm{B}}
$$

where $Z_{T}$ and $m_{\text {metal }}$ represent the atomic valence and magnetic moment in the pure $T$ metal $\left(Z_{T}=9\right.$ and $m_{\text {metal }}=1.7 \mu_{B}$ for Co $)$. Strong reduction of $m_{\text {tot }}$ is expected when $M$ is a nonmagnetic element. Comparison of this theoretical prediction with the values obtained for our Co-W NPs is shown in Fig. 2.20. Although there is a qualitative agreement in the trends, we find a significant deviation from the predicted slope, which can be attributed to the limitations of the model that takes insufficient account of the atomic structure, nearest-neighbor environments and chemical disorder [105].

In Co amorphous magnetic alloys, which have local anisotropy at the Co sites with randomly oriented local easy axes, the Co-Co exchange interaction is of sufficient intensity to guarantee collective ferromagnetic order if the Co content is above a critical value $[105]$. In Co-W alloys it is $\sim 76 \%$ at. Co, 


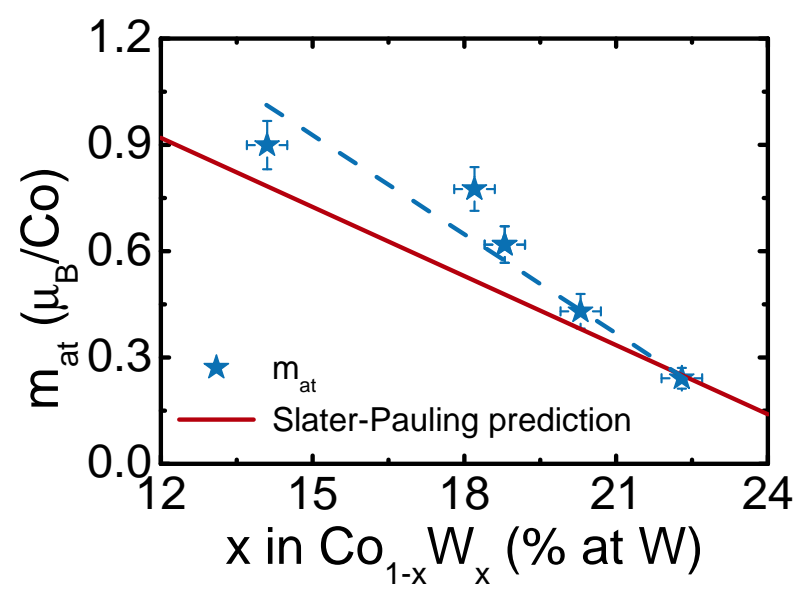

Figure 2.20: Dependence of $m_{\text {tot }}$ with the composition of the Co-W alloy in the NPs. (Dashed line) Guide to the eye.

so that those alloys with smaller values have no magnetic moment $[26\rfloor$. In general, for $3 d$-metal based alloys, the orientational correlation length of the local magnetic moments has been estimated to be of the order of a few $\mu \mathrm{m}$ 112. Therefore, in Co-W nanoparticles, with a diameter of the order of a few nanometers, exchange stiffness can maintain local moment correlation within the whole particle, and thus, yield to a particle net average moment and anisotropy.

Amorphous magnetic alloys are expected to behave magnetically isotropic or to exhibit a low anisotropy (in the case of thin films where a stress-induced anisotropy can be present) given their lack of atomic long range order. In contrast, in our Co-W NPs $K_{\text {eff }}$ is all but negligible. Its origin may be traced back to the structural or chemical SRO we have shown to exist in section 2.2.2. Indeed, some amorphous alloys, typically following the formula $T_{1-x}-M_{x}$, still exhibit remarkable SRO $\lfloor 92,103\rfloor$ Coordination numbers of about 12 found by structural characterization of these alloys may be visualized as $h c p$ or $f c c$ like [103. Such amorphous materials are often simulated by small structural units, which are defined by a reference atom and its nearest neighbors. The structural model should contain sites having $h c p$ and $f c c$ like symmetries and are modeled as icosahedral, octahedral and trigonal units [92 . Given the presence of SRO, large local magnetic anisotropies, comparable to those of crystalline Co, for example, are expected. Moreover, if the anisotropy is due to chemical SRO, $K_{\text {eff }}$ is expected to increase linearly with the W concentration [88], contrary to our observation. The origin of $K_{\text {eff }}$ in these materials is then structural. Values of $K_{\text {eff }}$ on the order of the $10^{5} \mathrm{erg} / \mathrm{cm}^{3}$ have been reported for amorphous $\mathrm{Co}_{1-x} \mathrm{Ti}_{x}$ films [87] and on the order of $10^{6} \mathrm{erg} / \mathrm{cm}^{3}$ for amorphous $\mathrm{Co}_{1-x} \mathrm{Zr}_{x}$ films [88]. The latter is comparable to those obtained for our amorphous 
Co-W alloy NPs. Such high values of magnetic anisotropy are attributed to the strong local uniaxial anisotropy of the clusters $[87,88]$. In $\mathrm{Co}_{1-x} M_{x}$ amorphous alloys $(M=\mathrm{Ti}$ and $\mathrm{Zr})$, clusters formed of only Co atoms are magnetically isotropic, while those where the Co atom possess a number of $M$ atoms as nearest neighbors present a local anisotropy, so submitted to greater anisotropy energy. The local anisotropy depends on both the local symmetry of the Co atoms and to the saturation magnetization, $M_{\mathrm{S}}[88]$. If the local disorder increases by increasing the amount of $M$ atoms, the clusters are less defined giving rise to a reduction in $K_{\text {eff }}$. Such dependence of $K_{\text {eff }}$ with $x$ has been observed in $\mathrm{Co}_{1-x} \mathrm{Ti}_{x}$ [87」 and $\mathrm{Co}_{1-x} \mathrm{Zr}_{x}$ films [88, and is also observed in our Co-W NPs (see Fig. 2.21).

In Fig. 2.22, the $K_{\text {eff }}$ and $m_{\mathrm{L}} / m_{\mathrm{S}}$ results found for the amorphous CoW NPs are depicted together with the values found for bulk Co, and NPs of the same average diameter $(\mathrm{D} \sim 3 \mathrm{~nm})$; the bare Co NPs $\lfloor 11\rfloor$ and, for completeness, the published values for alloyed Co-Pt particles are included $\lfloor 22\rfloor$. In all these cases hybridization effects between $3 d$ Co and $5 d$ metal bands are at the origin of the increase of anisotropy. Thus, alloying Co with W increases the anisotropy with respect to bulk Co where crystalline anisotropy

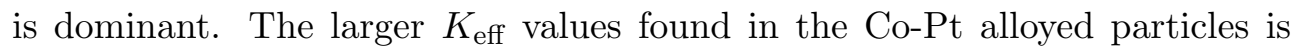
due to the larger spin-orbit coupling constant in the $\mathrm{Pt}$ case with respect to the $\mathrm{W}$ one $\left(\xi_{5 d}=0.66\right.$ and $0.038 \mathrm{eV}$ for $\mathrm{Pt}\lfloor 113\rfloor$ and $\mathrm{W}\lfloor 114\rfloor$, respectively). However, the enhancement driven by surface effects observed in the bare pure Co NPs is significantly larger [11].

Besides, when plotting $K_{\text {eff }}$ vs. the diameter of the Co-W particles (Fig. 2.23 ) we find an increase of $K_{\text {eff }}$ with increasing the NP diameter, instead of

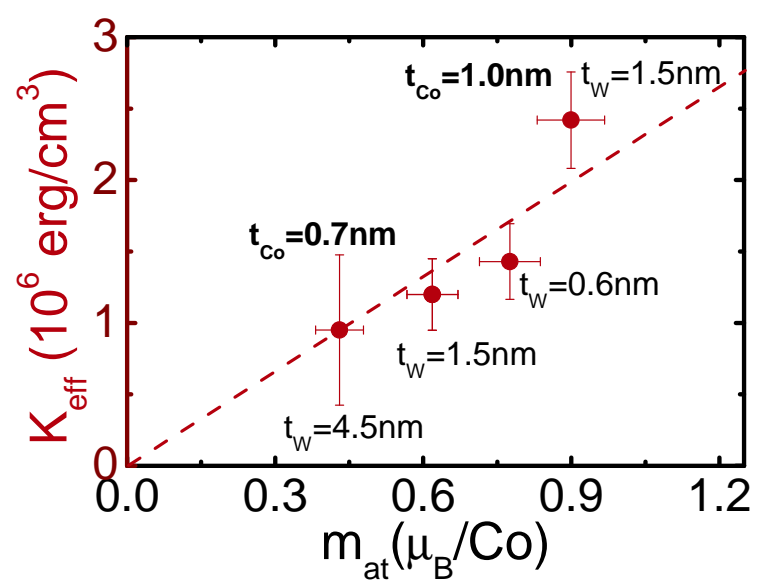

Figure 2.21: Dependence of $K_{\text {eff }}$ with $m_{\text {tot }}$ for all Co-W NPs samples. (Dashed line) Guide to the eye. 


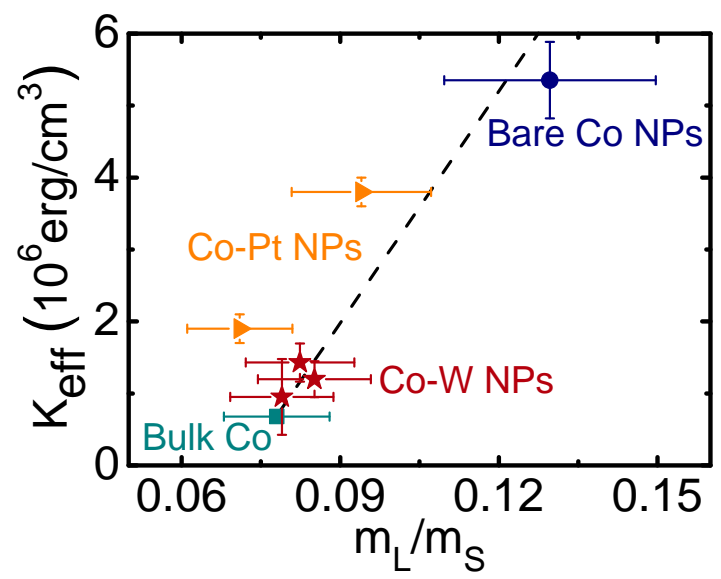

Figure 2.22: Dependence of $K_{\text {eff }}$ with $m_{\mathrm{L}} / m_{\mathrm{S}}$ for the $a$ series of Co-W samples and comparison with those for the bulk Co $\lfloor 107\rfloor$, bare Co NPs with $t_{\mathrm{Co}}=0.7 \mathrm{~nm}\lfloor 11$, and Co-Pt NPs with comparable diameters 22!. (Dashed line) Guide to the eye.

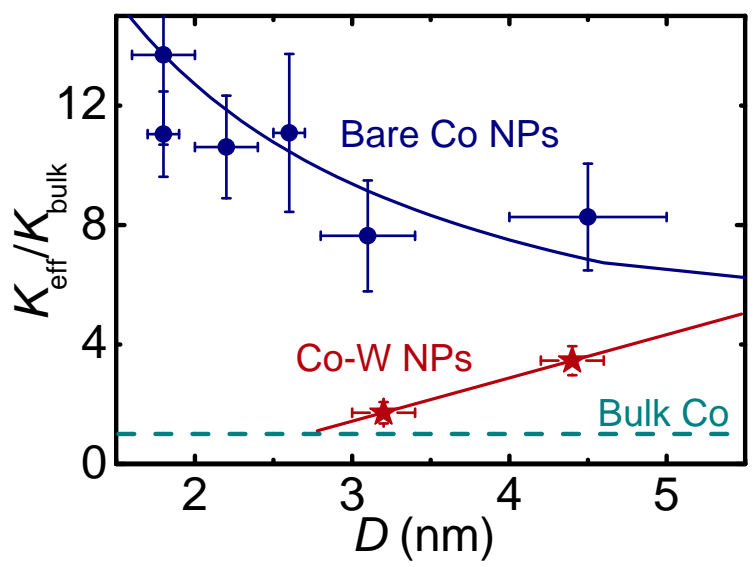

Figure 2.23: Average magnetic anisotropy normalized to its bulk value $\left(K_{\text {bulk }}=7\right.$ $\left.\times 10^{5} \mathrm{erg} / \mathrm{cm}^{3}\right)\lfloor 115\rfloor$ as a function of the Co-W particle diameter for the $b$ samples series along with the values for bare Co NPs $\lfloor 19$.

the $1 / D$ dependence found for the bare Co NPs $[19]$. This feature implies that there is an increase in the intrinsic anisotropy of the alloy, while the surface anisotropy is zero, as occurs, for example, in Co-Pt alloy NPs $\lfloor 22\rfloor$.

When comparing the magnetic behavior of our amorphous Co-W NPs with some other amorphous NPs systems studied in the literature, we find a quite different magnetic behavior. Most of the amorphous Fe NPs $171,72,74\rfloor$, Fe oxides $[73,75\rfloor$, organic coated Fe NPs $[76,77\rfloor$, amorphous Ni powder [82], Fe-

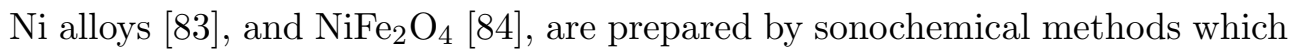
lead to agglomeration of the particles. Thus, in such systems, the magnetic 
interactions between particles play an important role. Instead, our Co-W NPs system constitute an ideal example of uniform non-interacting amorphous NPs well dispersed in an insulating matrix. Properties of the amorphous Fe and Ni based NPs systems are also those of superparamagnetic particles, with blocking temperature depending on the particle size $[71-77,82-84\rfloor$. In the organic coated Fe NPs, the surface dominates their magnetic properties since there is a chemical bonding between the functional groups attached to the particle and the Fe atoms $\left\lfloor 76,77\right.$. The chemical bonding modifies their $T_{B}$ and saturation magnetization, $M_{S}$. However, in the case of our Co-W NPs we have demonstrated that surface contribution is not as important as in those particulate systems.

Amorphous boride NPs with formula TM-B (with TM being one or two transition metals) show superparamagnetic behavior, interpreted in the frame of a core-shell model $\mid 78-81\rfloor$. Such particles are prepared by chemical synthesis and dispersed in a polymeric matrix for measurements. Opposite to our CoW NPs, TM-B have a distinct high temperature blocking temperature $T_{B}$, assigned to blocking of the ferromagnetically ordered core spins, and a second one at lower temperature, explained as due to blocking of the disordered shell spins. Besides, the $M(H)$ curves were temperature dependent; i.e., they did not follow a corresponding state law with $H / T$, on one hand, and had two components, a typical Langevin dependence, contributed by the core, and a linear dependent contribution, contributed by the spin disordered shell, on the other. In contrast, our Co-W NPs do fulfill the corresponding state law and such core-shell model (each with a different magnetic contribution) is not applicable.

In other Co-W multilayer systems the origin of the magnetic anisotropy observed is ascribed to the surface of the Co layers $\lfloor 116,117\rfloor$ and to volume-type strain anisotropy $\lfloor 116\rfloor$. However, we have demonstrated that interface effects play no role in the increase of $K_{\text {eff }}$ obtained for our Co-W NPs, compared to the bulk Co value.

Our Co-W NPs have an oblate shape with semiaxes $a=\left\langle D_{\text {eff }}\right\rangle_{\mathrm{PV}} / 2$, and $c=\left\langle D_{\text {eff }}\right\rangle_{\mathrm{CS}} / 2$, albeit with a small $c / a$ ratio; in the two samples for which the plan view and cross section average diameter could be determined (see table 2.1 ), $c / a \approx 0.93(1)$. Then, one may estimate the shape anisotropy constant $\lfloor 118\rfloor$ as:

$$
K_{\mathrm{sh}}=\frac{1}{2}\left(N_{a}-N_{c}\right) M_{\mathrm{sb}}^{2}
$$

where the demagnetization factors for that $c / a$ ratio are $N_{a} \approx 0.325$ and $N_{c} \approx 0.350\lfloor 119\rfloor$, for the long and short particle semiaxes, and $M_{\mathrm{sb}}$ is the 
magnetization density of the particle. For the two particles with $t_{\mathrm{Co}}=0.7 \mathrm{~nm}$ and $t_{\mathrm{W}}=0.6$ and $1.5 \mathrm{~nm}, M_{\mathrm{sb}}=0.413$ and $0.367 \times 10^{3} \mathrm{emu} / \mathrm{cm}^{3}$, respectively (see table 2.3 ), which yields $K_{\mathrm{sh}}=-2.0$ and $-1.7 \times 10^{3} \mathrm{emu} / \mathrm{cm}^{3}$, respectively. These values are nearly three orders of magnitude smaller than the anisotropy constant found from our a.c susceptibility measurements, so, we may consider this contribution as negligible.

Then, shape anisotropy cannot explain the $K_{\text {eff }}$ found for our Co-W NPs. Stress anisotropy is also one order of magnitude smaller [105. Instead, the $\mathrm{SRO}$ detected in our amorphous Co-W alloy NPs is probably the main factor in the origin of their magnetic anisotropy.

\subsection{Conclusions}

We have presented a complete study on a system of self-organized amorphous $\mathrm{Co}-\mathrm{W}$ alloy nanoparticles, uniformly dispersed in an alumina matrix. The size and composition of the Co-W alloy NPs have been controlled by varying the amount of Co or $\mathrm{W}$ in the system. Such parameters strongly affect their structural and magnetic properties. Co-W hybridization and electronic transfer from $\mathrm{W} 5 d$ to Co orbitals have been observed by XANES measurements, so that as the $\mathrm{Co} / \mathrm{W}$ ratio in the samples increases, there is more electronic transfer from $\mathrm{W} 5 d$ towards Co $4 p$ bands and less delocalization of Co $3 d$ empty states. The amorphous nature of the Co-W NPs was verified by HRTEM and EXAFS measurements. The particles are superparamagnetic with minimal interactions between them, as demonstrated by the FC-ZFC magnetic measurements. Their magnetism is governed by the Co present in the alloy particles, so we were able to obtain values of the Co magnetic moment in the system via conventional SQUID magnetometry and compare it to results from XMCD measurements. A reduction of the Co moment per Co atom present in the samples was observed, compared to the bulk Co value. We have explained such decrease as due to the reduction of Co coordination in the alloy, which was verified by EXAFS and has been described in the literature. SRO in the amorphous Co-W alloy NPs is evident and is responsible for the magnetic anisotropy detected in the particles, contrary to other amorphous nanoparticles systems where the anisotropy is due to surface effects. With this work, we supply a paradigmatic example in the phenomenological body of magnetic nanoparticle behavior. 


\section{Chapter 3}

\section{Breakdown of Hund's third rule in amorphous Co-W alloy nanoparticles}

In the previous chapter, we have demonstrated that the amorphous $\mathrm{Co}-\mathrm{W}$ alloy NPs constitute a paradigmatic system among the specific group of magnetic nanoparticles with amorphous structure: contrary to other amorphous nanoparticle systems where the anisotropy is due to surface effects, its origin in the amorphous Co-W alloy NPs lies on the structural short range order (SRO) detected in the particles. We have also described the Co-W hybridization and electronic transfer from W $5 d$ to Co orbitals in these Co-W NPs by analysis of X-ray absorption near edge structure (XANES) measurements at the Co $K$ and $\mathrm{W} L_{3}$ edges (the electron configuration for $\mathrm{Co}$ is $[\mathrm{Ar}] 3 d^{7} 4 s^{2}$ and for $\mathrm{W}$ is $[\mathrm{Xe}] 4 f^{14} 5 d^{4} 6 s^{2}$ ). The electronic transfer from $\mathrm{W} 5 d$ toward Co $4 p$ bands, and the delocalization of the Co $3 d$ empty states were qualitatively described as a function of the $\mathrm{Co} / \mathrm{W}$ ratio in these Co-W NPs samples.

The effects of similar $3 d-5 d$ hybridization, in particular on $5 d$ induced magnetic moments, have been previously studied both experimentally and theoretically in $\mathrm{Fe} / \mathrm{W}$ multilayers $\left\lfloor 120\right.$. and bulk $\mathrm{Co}_{100-x} \mathrm{Ir}_{x}$ alloys $\lfloor 70\rfloor$, to cite some examples of metallic systems. A remarkable finding in these two systems is the observation of a breakdown of Hund's third rule of the induced orbital and spin magnetic moments in the $5 d$ metals. This rule states how spin and orbital total moments in a single atom should be aligned, being parallel for an atom with more than half-filled shell, and antiparallel for a less than half-filled shell [121]. In spite of its phenomenological character and of being defined for single isolated atoms, atoms in solids have been proven to ubiquitously follow this rule, too. 
However, the two cases mentioned above are part of the few examples where this rule is not completely fulfilled $\lfloor 70,120,122-124\rfloor$. In the Fe/W multilayers, the total spin and orbital magnetic moments of the $5 d \mathrm{~W}$ shell couple in parallel (notice that $\mathrm{W}$ is a less than half-filled $5 d$ band metal), and for Ir in bulk Co-Ir alloys they are antiparallel aligned (Ir is a more than halffilled $5 d$ band metal), being, in both cases, contrary to the predictions of the Hund's third rule $[70,120\rfloor$. All these experimental findings have been possible thanks to the highly sensitive X-ray magnetic circular dichroism (XMCD) technique, which, as described in previous chapters, allows to perform element specific magnetometry, as well as to separate the contribution of the orbital and spin moments to the total magnetic moment and their relative orientation, by applying the magneto-optical sum rules $[64,65\rfloor$.

The structural disorder in transition metal alloys has also been demonstrated to influence their electronic configuration $\lfloor 123\rfloor$. In fact, for amorphous Fe-Zr and Co-Zr alloy films, the induced spin and orbital moments detected in $\mathrm{Zr}$ (a less-than half filled $4 d$ element) are found to be parallel aligned, thus breaking down the Hund's third rule. However, for similar crystalline compounds Fe-Zr-Pt and Co-Zr-Pt, the induced orbital and spin moments in $\mathrm{Zr}$ are found to fulfill this rule.

Following these results, the amorphous Co-W alloy NPs arise as an attractive system to investigate the hybridization between Co orbitals and the $5 d$ band of $\mathrm{W}$, and its consequence on the induced spin and orbital magnetic moments in W. XANES and XMCD are the best techniques to study the electronic transfer between Co and $\mathrm{W}$, and examine the compliance or breakdown of the Hund's third rule in the $\mathrm{W}$ atoms in Co-W systems. This chapter is then dedicated to delving into these hybridization effects, both experimentally by XANES and XMCD measurements at the Co $K$ and $\mathrm{W} L_{2,3}$ edges, and theoretically by simulations of the XANES and XMCD signals at both edges in the amorphous Co-W alloy NPs system. The polycrystalline bulk $\mathrm{Co}_{3} \mathrm{~W}$ alloy is also included in the present study, so that, by comparison to the amorphous NPs, the influence of the structural disorder is evaluated.

\subsection{XANES and XMCD measurements}

XANES and XMCD measurements at the Co $K(7709 \mathrm{eV}), \mathrm{W} L_{3}(10207$ $\mathrm{eV})$ and $\mathrm{W} L_{2}(11544 \mathrm{eV})$ edges on the Co-W NPs samples and the bulk $\mathrm{Co}_{3} \mathrm{~W}$ alloy were performed at the ESRF ID12 beamline. Spectra for $\mathrm{Al}_{2} \mathrm{O}_{3}-$ capped Co NPs and on a W metallic foil for reference, were also recorded, at the Co $K$ and W $L_{2,3}$ edges, respectively. The APPLE-II undulator and a double - Si - (111) - crystal monocromator were used to collect the spectra 
at the respective energies. XANES spectra were recorded by a fluorescence detector in backscattering geometry. XMCD signal was obtained by applying a magnetic field of 10 and $50 \mathrm{kOe}$ for the $\mathrm{Co}-\mathrm{W}$ NPs and the $\mathrm{Co}_{3} \mathrm{~W}$ alloy, respectively, normal to the sample plane and along the X-ray beam direction. Measurements were performed at 7 and $10 \mathrm{~K}$ for the Co-W NPs and the $\mathrm{Co}_{3} \mathrm{~W}$ alloy, respectively. This field and temperature were chosen according to the magnetic properties of the Co-W NPs, so that the system was reaching its magnetic saturation under these conditions (see section 2.3.1). XMCD was obtained by differences of XANES spectra measured with opposite helicities of the light at a fixed magnetic field value, orienting the field in two inverse directions. Polarization of the circular light was over $90 \%$ at the Co $K$ edge and $94 \%$ at the $\mathrm{W} L_{2,3}$ edges. Due to the low XMCD signal in W, several spectra were taken for each sample, being 16 spectra in average for each direction of the field. Due to experimental difficulties, the Co-W NPs sample with $t_{\mathrm{Co}}=$ $0.7 \mathrm{~nm}$ and $t_{\mathrm{W}}=4.5 \mathrm{~nm}$ was not measured at the $\mathrm{W} L_{2,3}$ edges.

The absorption spectra and XMCD for the bulk $\mathrm{Co}_{3} \mathrm{~W}$ alloy were corrected for self-absorption effects after their normalization of the absorption jump to unity. These corrections take into account that the sample is infinitely thick, and uses its chemical composition and density, along with some geometrical parameters of the measurement setup such as the angle of incidence of the X-ray beam and the solid angle of the detector, to estimate the effect of selfabsorption from the known energy dependence of the absorption coefficient of the sample. Details of the correction procedure can be found in Ref. [125. and references therein.

\subsubsection{Co $K$ edge results}

XANES and XMCD at the Co $K$ edge probes the $4 p$ empty states of the Co atom in the sample. Analysis of the XANES spectra recorded at this edge on these Co-W alloy NPs samples was described in section 2.2.2. Evidences of Co $4 p-3 d$ and Co $3 d-\mathrm{W} 5 d$ hybridization in the Co-W system and electronic transfer from W $5 d$ to Co orbitals were then observed. These findings were obtained not only from the observed changes of the Co $K$ edge XANES on the Co-W alloy NPs with respect to that of metallic Co, but also from the reduction of the magnetic moments of the Co atoms obtained in the Co-W alloy NPs by W inclusion in the alloy, as proven by analysis of the XMCD signals at the Co $L_{2,3}$ edges in section 2.3.3.

The detection of a nonzero XMCD signal at the Co $K$ edge on all samples, shown in Fig. 3.1, reflects the magnetic polarization of the $4 p$ band in Co atoms. In Fig. 3.1 we compare the XMCD spectra for all studied samples, 
normalized to the energy jump of the absorption edge: the $\mathrm{Al}_{2} \mathrm{O}_{3}$ capped Co NPs (Fig. 3.1(a)), the $a$ and $b$ samples series (Fig. 3.1(b) and (c), respectively), and the bulk $\mathrm{Co}_{3} \mathrm{~W}$ alloy (Fig. 3.1(d)). The origin of the energy scale has been chosen at the inflection point of the absorption edge. The XMCD signal in the case of the $\mathrm{Al}_{2} \mathrm{O}_{3}$ capped Co NPs (Fig. 3.1(a)) emerges by interatomic exchange interaction between the $4 p$ band of the absorbing Co and the $3 d$ bands of the neighboring Co atoms. This results from the allowed symmetry operations at the Co site in this cubic structure, so that the $4 p-3 d$ bands

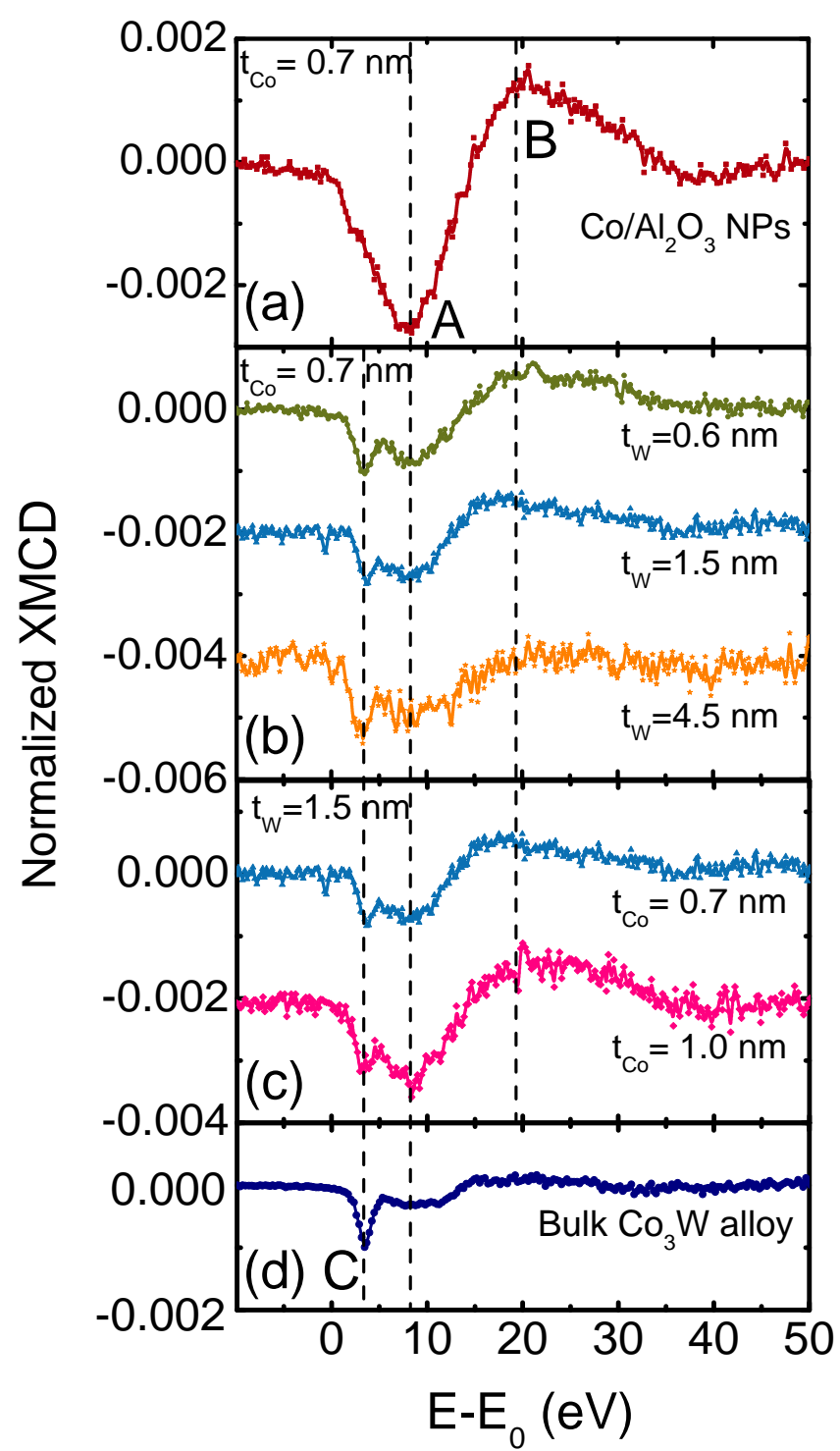

Figure 3.1: XMCD spectra at the Co $K$ edge for (a) $\mathrm{Al}_{2} \mathrm{O}_{3}$ capped Co NPs, (b) Co-W NPs $a$ sample series, (c) Co-W NPs $b$ sample series, and (d) bulk $\mathrm{Co}_{3} \mathrm{~W}$ alloy. 
mixing is only possible via the neighboring Co atoms $\lfloor 126\rfloor$. In the Co-W alloys (Fig. 3.1(b) to $(\mathrm{d})$ ), with a hexagonal local structure, this signal originates at the intra-atomic exchange interaction between the $4 p$ and $3 d$ bands in Co. The sign of the XMCD signal in all cases is the same as in Ref. $\mid 127$, indicating an antiferromagnetic coupling between $4 p$ and $3 d$ levels in Co.

Visible differences are observed in the Co $K$ edge XMCD spectra of the $\mathrm{Co}-\mathrm{W}$ NPs with respect to that of $\mathrm{Al}_{2} \mathrm{O}_{3}$ capped (see Fig. 3.1(a)); however, they are very similar to that of the $\mathrm{Co}_{3} \mathrm{~W}$ alloy (Fig. 3.1(d)). The dichroic signal in the $\mathrm{Al}_{2} \mathrm{O}_{3}$ capped Co NPs consists of a negative one-peak structure between 5 and $10 \mathrm{eV}$ above the edge (marked in Fig. 3.1(a) as peak A), followed by a positive large bump between $10 \mathrm{eV}$ and $30 \mathrm{eV}$ (denoted in Fig. 3.1(a) as peak B), similar to what is observed in metallic Co $\lfloor 126\rfloor$ and other metal capped Co NPs [11]. Instead, in our Co-W NPs (Fig. 3.1(b) and (c)) and in the $\mathrm{Co}_{3} \mathrm{~W}$ alloy (Fig. 3.1(d)) the broad negative peak $\mathrm{A}$ is split, giving rise to a double-peak structure formed by a trace of peak $\mathrm{A}$ and an additional negative peak, tagged as $\mathrm{C}$ in Fig. 3.1(d). This peak $\mathrm{C}$ is more pronounced in the XMCD signal of the bulk $\mathrm{Co}_{3} \mathrm{~W}$ alloy. A similar shape of Co $K$ edge XMCD signal has been observed by Rueff et al. [126」 in $\mathrm{LaCo}_{5}$ alloys, where they discussed the effect of the rare earth on the Co $K$ XMCD signal shape. They ascribe this extra feature in the negative peak to a contribution of the La $d$ empty states. Then, we may conjecture that this splitting in the Co-W systems is due to the $\mathrm{W} 5 d$ states in the Co- $\mathrm{W}$ alloy.

\subsubsection{W $L_{2,3}$ edges results}

XANES spectra recorded at the $\mathrm{W} L_{2,3}$ were normalized as $L_{3} / L_{2}$ to $2.19 / 1$,

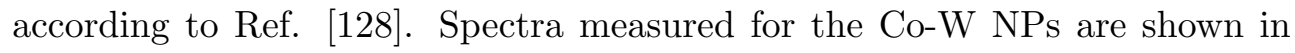
Fig. 3.2(a), along with the one for a metallic $\mathrm{W}$ foil. XANES of the bulk $\mathrm{Co}_{3} \mathrm{~W}$ alloy is plotted in Fig. 3.3(a). Changes in the absorption white-line at $\mathrm{W} L_{2,3}$ edges for the Co-W NPs are observed and reflect variation of the available states of $5 d$ character. Specifically, $L_{3}$ absorption edge appears for transitions from $2 p$ to both $5 d_{3 / 2}$ and $5 d_{5 / 2}$, while $L_{2}$ reveals only transitions from $2 p$ to $5 d_{3 / 2}$ band. A qualitative analysis of this variation was described in section 2.2.2. We observed that an increase in the $\mathrm{Co} / \mathrm{W}$ ratio in the $\mathrm{Co}-\mathrm{W}$ NPs samples gave rise to an increase in the number of W $5 d$ empty states, $n_{h}^{\mathrm{W}}$; i.e., there is an electron transfer from $\mathrm{W}$ toward Co. The change in $n_{h}^{\mathrm{W}}$ for each sample may be quantified by analysis of the $\mathrm{W} L_{2,3}$ XANES spectra. The integral of the white line at the $L_{2,3}$ edges of each sample, $r_{\mathrm{Co}-\mathrm{W}}$, and that for W foil, $r_{\text {Wfoil }}$, are defined as the sum of the individual $L_{2}$ and $L_{3}$ integrals, $I_{L 2}$ and $I_{L 3}$, respectively, calculated by first subtracting the continuum from each XANES. The continuum is artificially represented by a double-step function. 


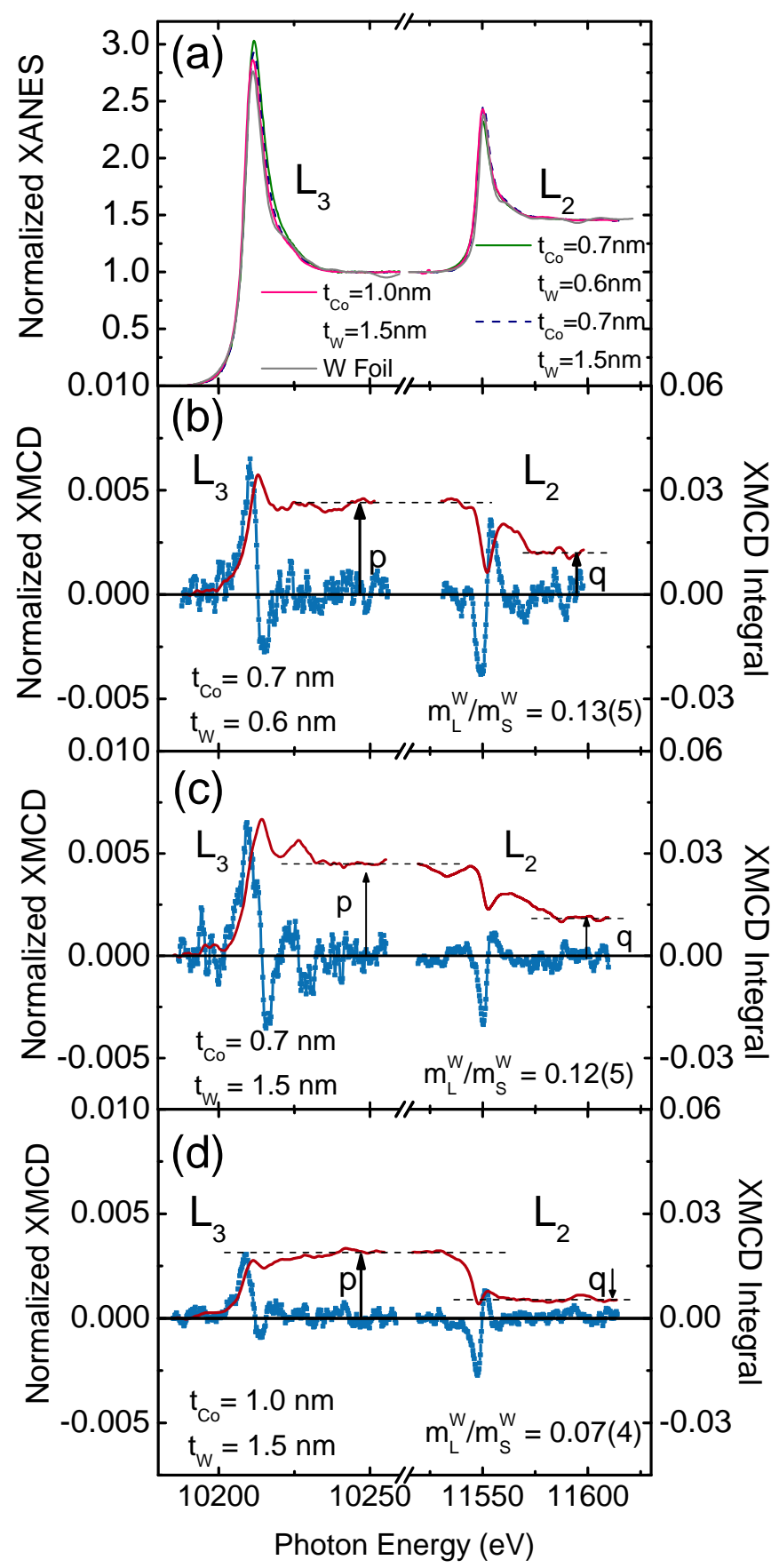

Figure 3.2: (a) XANES spectra at the $\mathrm{W} L_{2,3}$ for the Co-W NPs, and for a W reference foil. (b) to (d) show normalized XMCD signal and XMCD integral for each Co-W alloy NPs sample at the $\mathrm{W} L_{2,3}$ measured at $H=10 \mathrm{kOe}$. Arrows indicate the energy at which $p$ and $q$ integrals were determined. 


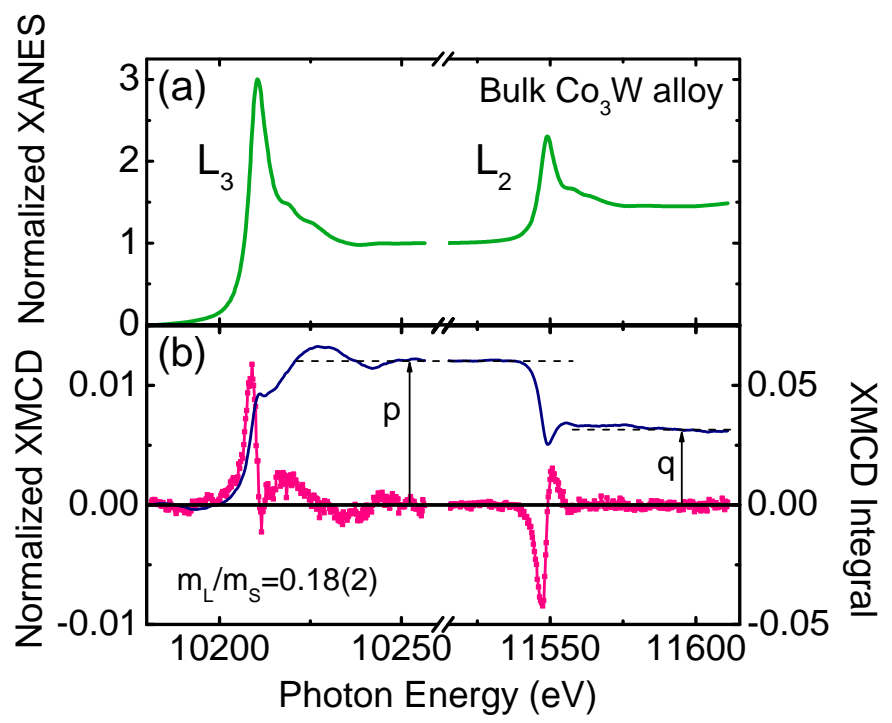

Figure 3.3: (a) XANES spectra and (b) XMCD signal and XMCD integral at the W $L_{2,3}$ for the $\mathrm{Co}_{3} \mathrm{~W}$ alloy measured at $H=50 \mathrm{kOe}$ and $T=10 \mathrm{~K}$. Arrows indicate the energy at which $p$ and $q$ integrals were determined.

This double - step approach is extensively used for the $3 d$ FM metals, and its accuracy in the case of $\mathrm{W}$ has been previously estimated to be about $94 \%$, given its relative large white line intensity $\lfloor 120$. Then, assuming that for metallic $W$

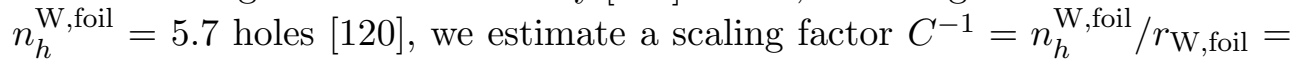
0.1936 holes/eV. Finally, the number of holes for each Co-W sample can be calculated as $n_{h}^{\mathrm{W}}=C^{-1} r_{\mathrm{Co}-\mathrm{W}}$.

Results for these calculations for both the Co-W alloy NPs and the bulk $\mathrm{Co}_{3} \mathrm{~W}$ alloy are listed in table 3.1. From these values we observe that the number of holes in $\mathrm{W}$ in the Co-W alloy NPs slightly increases with respect to that in metallic $\mathrm{W}$, and to that found for the bulk $\mathrm{Co}_{3} \mathrm{~W}$ alloy, due to hybridization of the $5 d$ band in $\mathrm{W}$ with the $3 d$ band in Co. Moreover, we notice a further increase in $n_{h}^{\mathrm{W}}$ when the amount of Co in the sample increases, and when $t_{\mathrm{W}}$ decreases. This means that the electronic transfer from the $5 d$ band in $\mathrm{W}$ to the $3 d$ band in Co depends on the $\mathrm{Co} / \mathrm{W}$ proportion in the samples. For the $\mathrm{Co}_{3} \mathrm{~W}$ alloy a slight increase in $n_{h}^{\mathrm{W}}$ with respect to the value of metallic $\mathrm{W}$ is also observed.

The ratio between the unoccupied states in the $5 d_{5 / 2}$ and $5 d_{3 / 2}$ bands in $\mathrm{W}, n_{h}^{5 / 2} / n_{h}^{3 / 2}$, may be evaluated from the branching ratio $I_{L 3} / I_{L 2}$, following the analysis described in Ref. [129]. The results of $n_{h}^{5 / 2} / n_{h}^{3 / 2}$ for the Co-W NPs and the bulk $\mathrm{Co}_{3} \mathrm{~W}$ alloy are given in table 3.1. Similar to $n_{h}^{\mathrm{W}}$, we observe that this ratio varies depending on the $\mathrm{Co} / \mathrm{W}$ proportion in the sample; i.e., 
Table 3.1: Values of $x$ in the $\mathrm{Co}_{1-\mathrm{x}} \mathrm{W}_{\mathrm{x}}$ alloy (values extracted from table 2.3), $n_{h}^{\mathrm{W}}$, $n_{h}^{5 / 2} / n_{h}^{3 / 2}$ ratio, and $m_{\mathrm{L}} / m_{\mathrm{S}}$ ratio as calculated from the XMCD sum rules for the $\mathrm{Co}-\mathrm{W}$ NPs samples in the present study and the bulk $\mathrm{Co}_{3} \mathrm{~W}$ alloy. $n_{h}^{\mathrm{W}}=5.7$ holes for metallic $\mathrm{W}\lfloor 120\rfloor$.

\begin{tabular}{cccccc}
\hline \hline \multicolumn{2}{c}{ Sample } & $x$ & $n_{h}^{\mathrm{W}}$ & $n_{h}^{5 / 2} / n_{h}^{3 / 2}$ & $m_{\mathrm{L}} / m_{\mathrm{S}}$ \\
\hline \multicolumn{6}{c}{ Co-W NPs } \\
$t_{\mathrm{Co}}(\mathrm{nm})$ & $t_{\mathrm{W}}(\mathrm{nm})$ & & & & \\
1.0 & 1.5 & $14.1(4)$ & $6.09(3)$ & $0.50(1)$ & $0.07(4)$ \\
0.7 & 0.6 & $18.2(4)$ & $6.06(5)$ & $0.52(1)$ & $0.13(5)$ \\
0.7 & 1.5 & $18.8(4)$ & $6.00(5)$ & $0.54(1)$ & $0.12(5)$ \\
Bulk $\mathrm{Co}_{3} \mathrm{~W}$ & $25(1)$ & $5.85(2)$ & $0.59(1)$ & $0.18(5)$ \\
Calc. $\mathrm{Co}_{3} \mathrm{~W}$ & 25 & $5.96(2)$ & $0.57(2)$ & $0.10(1)$ \\
\hline \hline
\end{tabular}

$n_{h}^{5 / 2} / n_{h}^{3 / 2}$ increases when the $\mathrm{Co} / \mathrm{W}$ ratio decreases, being highest for the bulk $\mathrm{Co}_{3} \mathrm{~W}$ alloy.

XMCD signals at the $\mathrm{W} L_{2,3}$ on each sample were normalized according to factors obtained from XANES normalization. Figures 3.2(b) to 3.2(d) show XMCD signals for each Co-W NPs sample, and Fig. 3.3(b) for the $\mathrm{Co}_{3} \mathrm{~W}$ alloy. The XMCD integrals at $L_{3}$ and $L_{2,3}$ edges, defined as $p$ and $q$, respectively, are also shown. The existence of a XMCD signal at W $L_{2,3}$ evidences the presence of an induced magnetic moment in the $\mathrm{W}$ atoms by the magnetic Co atoms in the Co-W alloy NPs and the $\mathrm{Co}_{3} \mathrm{~W}$ alloy. According to the direction of the applied magnetic field, and considering that the $L_{3} \mathrm{XMCD}$ signal is mostly positive, while the $L_{2}$ XMCD is negative, we can determine that the induced $\mathrm{W}$ magnetic moment orients opposite to the direction of the field. Consequently, $\mathrm{W}$ induced moments are antiparallel to the Co magnetic moments deduced for the Co-W NPs in section 2.3.3. This is consistent with the general trend in all transition metal series with the nd band filling: $\mathrm{W}$ has a less than half filled $5 d$ band, which results in antiparallel coupling of its induced moment with that of the magnetic $3 d$ metal $\lfloor 120,123\rfloor$.

Values of $p$ and $q$ were used to calculate the orbital to spin moment ratio, $m_{\mathrm{L}} / m_{\mathrm{S}}$, in the induced magnetic moments in $\mathrm{W}$ by applying the magnetooptical sum rules $\lfloor 64,65$. We have followed the symbol and sign criteria used in Ref. 66 , , so that the effective spin moment obtained from the sum rules is expressed as $m_{\mathrm{Seff}}=m_{\mathrm{S}}+m_{\mathrm{D}}$, where $m_{\mathrm{D}}$ represents the dipolar term, proportional to the expectation value of the magnetic dipole operator $\left\langle T_{z}\right\rangle$. Thus, in order to determine $m_{\mathrm{S}}$, one has to consider the $\left\langle T_{z}\right\rangle$ value. For alloys with a non-cubic structure, like the $h c p \mathrm{Co}_{3} \mathrm{~W}$ alloy, $\left\langle T_{z}\right\rangle$ might have a maximum value of $\left\langle S_{z}\right\rangle / 3$, corresponding to the Lorentz local field at the 
W site, as described for Ir in Co-Ir alloys [70]. Following this approximation, $m_{\text {Seff }} \leq 13 / 6 m_{\mathrm{S}}$.

Notwithstanding, in the case of our amorphous Co-W alloy particles or in the polycrystalline $\mathrm{Co}_{3} \mathrm{~W}$ sample, where their individual anisotropy axes are randomly oriented, the angle averaged $\left\langle T_{z}\right\rangle$ becomes negligible, thus we consider below that $m_{\mathrm{S}}=m_{\text {Seff }}$. Moreover, we refrain from calculating absolute values of $m_{\mathrm{S}}, m_{\mathrm{L}}$ and $m_{\mathrm{tot}}$ in the Co-W NPs because of the uncertainty of the actual amount of $\mathrm{W}$ in their $\mathrm{Co}-\mathrm{W}$ alloy, taking into account that not all of the $\mathrm{W}$ in the samples is alloyed with Co, as described in chapter 2.

$m_{\mathrm{L}} / m_{\mathrm{S}}$ results are listed in table 3.1. In all the Co-W systems in the present study $m_{\mathrm{L}} / m_{\mathrm{S}}$ in $\mathrm{W}$ is positive, which indicates that the orbital and spin moments in $\mathrm{W}$ are parallel aligned. Those values in the Co-W NPs samples are found to be nearly constant for a fixed amount of Co, and to decrease as the amount of Co in the sample increases. The latter may be understood as a reduction of the contribution of the orbital magnetism as the $\mathrm{Co} / \mathrm{W}$ ratio in the sample increases. Comparing $m_{\mathrm{L}} / m_{\mathrm{S}}$ values for the Co- $\mathrm{W}$ NPs with that of the $\mathrm{Co}_{3} \mathrm{~W}$ alloy we observe that the latter is higher. This may also be related to the $\mathrm{Co} / \mathrm{W}$ ratio on each alloy, as will be discussed in section 3.3 .

For the $\mathrm{Co}_{3} \mathrm{~W}$ alloy, application of the sum rules yields to values of $m_{\mathrm{S}}=$ $-0.023(2), m_{\mathrm{L}}=-0.004(1)$ and $m_{\mathrm{tot}}=-0.027(2) \mu_{\mathrm{B}} / \mathrm{W}$, for $n_{h}=5.85$ holes in $\mathrm{W}$ atoms. The total magnetic moment in $\mathrm{W}$ for this alloy is one order of magnitude lower than the $-0.2 \mu_{\mathrm{B}} / \mathrm{W}$ moment measured for $\mathrm{Fe} / \mathrm{W}$ multilayers in Ref. [120]. However, the $m_{\mathrm{L}} / m_{\mathrm{S}}$ ratio found in the $\mathrm{Co}_{3} \mathrm{~W}$ alloy is higher than in the experimental value reported for Fe/W multilayers [120], but comparable to that estimated by first principles calculations for the latter system [130].

\subsection{Calculations of DOS, XANES and XMCD}

Calculations of the electronic density of states (DOS) projected at the Co $3 d$ and $\mathrm{W} 5 d$ orbitals, as well as XANES and XMCD spectra at the Co $K$ and the $\mathrm{W} L_{2,3}$ edges in the $\mathrm{Co}_{3} \mathrm{~W}$ system have been performed in collaboration with Dr. Fernando Bartolomé in the Instituto de Ciencia de Materiales de Aragón, CSIC - Universidad de Zaragoza, by using the ab initio code FDMNES $\lfloor 131,132$. This program gives the absorption cross section of photons around the ionization edge using fully relativistic monoelectronic calculations (DFTLSDA). We have used the two different approaches for monoelectronic calculations that this code provides in order to simulate the X-ray absorption spec- 
tra: (i) a finite difference method to solve the Schrödinger equation, where the shape of the potential is free, thus a muffin-tin (MT) approximation can be avoided, and (ii) a Green formalism (multiple scattering) with a MT potential.

The structural and crystallographic information of the $\mathrm{Co}_{3} \mathrm{~W}$ system used to define the position of the absorbing and scattering atoms in the calculations is the one previously described in section 2.1. A cluster of $6 \AA$ radius was used and a Fermi level of about 7701, 10199, and $11536 \mathrm{eV}$ for the Co $K$, $\mathrm{W} L_{3}$ and $\mathrm{W} L_{2}$ edges, respectively. The initial parameters of the atomic electronic configurations of Co and $\mathrm{W}$ have been fixed as those obtained from the analysis of experimental XMCD (see W $5 d n_{h}$ in table 3.1). However, slightly modified electronic configurations were tested during the simulations in order to evaluate the dependence of the calculated XMCD signal with the initial moment in the Co and $\mathrm{W}$ nd bands, as will be described below.

\subsubsection{DOS calculations}

The spin polarized DOS projection at the Co $3 d$ and W $5 d$ orbitals for the $\mathrm{Co}_{3} \mathrm{~W}$ system were performed as described in Refs. $\lfloor 131,132\rfloor$, and are shown in Fig. 3.4. The traditional sign of hybridization $\lfloor 133\rfloor$ is observed on the curves; i.e., W $5 d$ states follow the main features of the Co $3 d$ DOS, specially in the energy region where the latter have their dominating weight.

The number of electrons in the $\mathrm{W} 5 d$ band were calculated by integration of the DOS in Fig. 3.4, up to the Fermi energy, $E_{f}$, resulting in $4.04(2)$

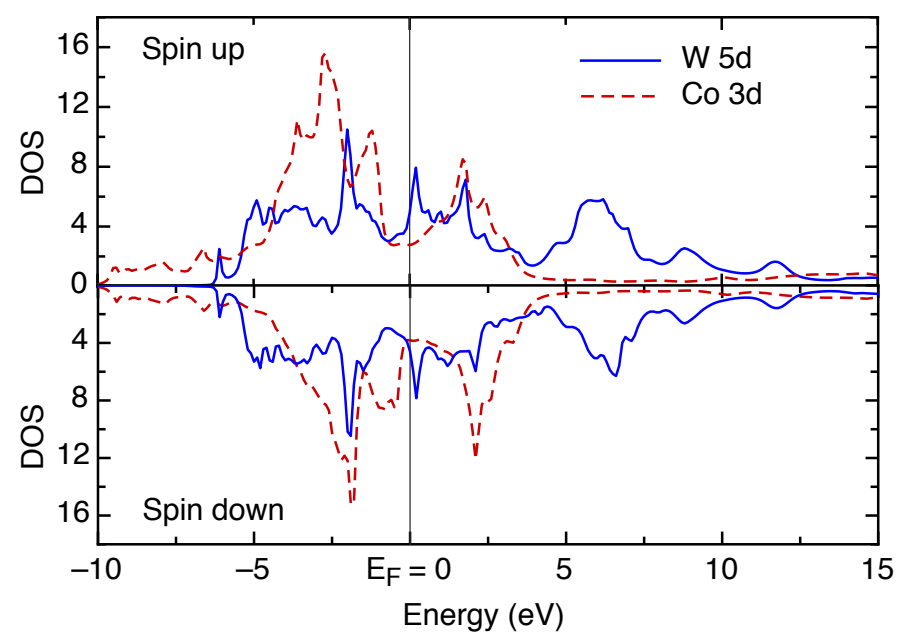

Figure 3.4: Calculated density of states (DOS) for the $\mathrm{Co}_{3} \mathrm{~W}$ system. DOS projected at the Co $3 d(--)$ and W $5 d(-)$ orbitals. Spin-up DOS are shown as positive and spin-down as negative. 
electrons, or equivalently, 5.96(2) holes. This result is very close to the $5.85(2)$ holes calculated for the $\mathrm{W} 5 d$ band from the $\mathrm{W} L_{2,3}$ XANES analysis in section 3.1.2. The difference between the number of electrons with spin up and down in the $\mathrm{W} 5 d$ band amounts to $0.014(1)$ electrons, which results in an estimated spin magnetic moment of $0.014(1) \mu_{B}$. This value is on the same order of the $m_{\mathrm{S}}$ derived for the $\mathrm{W}$ atoms from the sum rules applied to the $\mathrm{W} L_{2,3} \mathrm{XMCD}$ results in the $\mathrm{Co}_{3} \mathrm{~W}$ alloy in section 3.1.2. The difference between the two values may be an artifact of the calculation, due to the MT approximation on the form of the potential $\lfloor 131\rfloor$.

\subsubsection{Co $K$ edge}

The calculated XMCD signal at the Co $K$ edge for the $\mathrm{Co}_{3} \mathrm{~W}$ system is plotted in Fig. 3.5. A qualitative agreement between the simulated and the experimental curves for the $\mathrm{Co}_{3} \mathrm{~W}$ alloy is found both for XANES and XMCD.

The splitting of the negative peak observed in these Co-W systems at about $5 \mathrm{eV}$ above the edge (marked as peak $\mathrm{C}$ in Fig. 3.1 and Fig. 3.5(b)) is reproduced in the calculations although the relative intensities and energy positions may vary. In particular, the energy positions of the calculated features above the edge are affected by an overestimation of the interaction with the core-hole in the finite-difference method calculation (peaks A and B in Fig. 3.5(b) are shifted to lower energies), and, therefore, the agreement with the experiment can only be expected to be qualitative. In order to take insight of the origin of the different peaks of the spectrum, we have calculated XAS and XMCD of a fictitious " $\mathrm{Co}_{3} \mathrm{Co}$ " system with the $\mathrm{Co}_{3} \mathrm{~W}$ structure by substituting the W by $\mathrm{Co}$ in the definition of the cluster, and therefore, eliminating the presence of $5 d$ states in the calculation. The $\mathrm{Co}_{3} \mathrm{Co} \mathrm{XMCD}$ result is shown in Fig. 3.5(b) for comparison. Although the $\mathrm{Co} K$ edge XAS calculated in $\mathrm{Co}_{3} \mathrm{Co}$ is only slightly different than $\mathrm{Co}_{3} \mathrm{~W}$ at the pre-edge feature, the $\mathrm{Co}_{3} \mathrm{Co} \mathrm{XMCD}$ is not split into two negative peaks, but closely resembles the bulk Co XMCD, which is similar to the measurement recorded in bare Co NPs. The calculations confirm our conjecture (section 3.1.1) on the origin of the splitting of the Co $K$ XMCD edge, and therefore, we positively ascribe the first negative XMCD peak experimentally observed in $\mathrm{Co}_{3} \mathrm{~W}$ to the hybridization of $\mathrm{W} 5 d$ band states with the Co empty states probed in $K$ edge absorption. Nominally, those are the Co $4 p$ states through dipolar E1 transitions and the Co $3 d$ states through quadrupolar E2 ones. However, the calculated Co $K$ edge XMCD on $\mathrm{Co}_{3} \mathrm{~W}$ is split also when calculated in dipolar approximation only, showing that the hybridized $\mathrm{W} 5 d$ band is probed also at the Co $4 p$ states. Moreover, the correct sign of the calculated split XMCD feature (Fig. 3.5b) is only achieved for the (experimentally observed) antiparallel configuration be- 

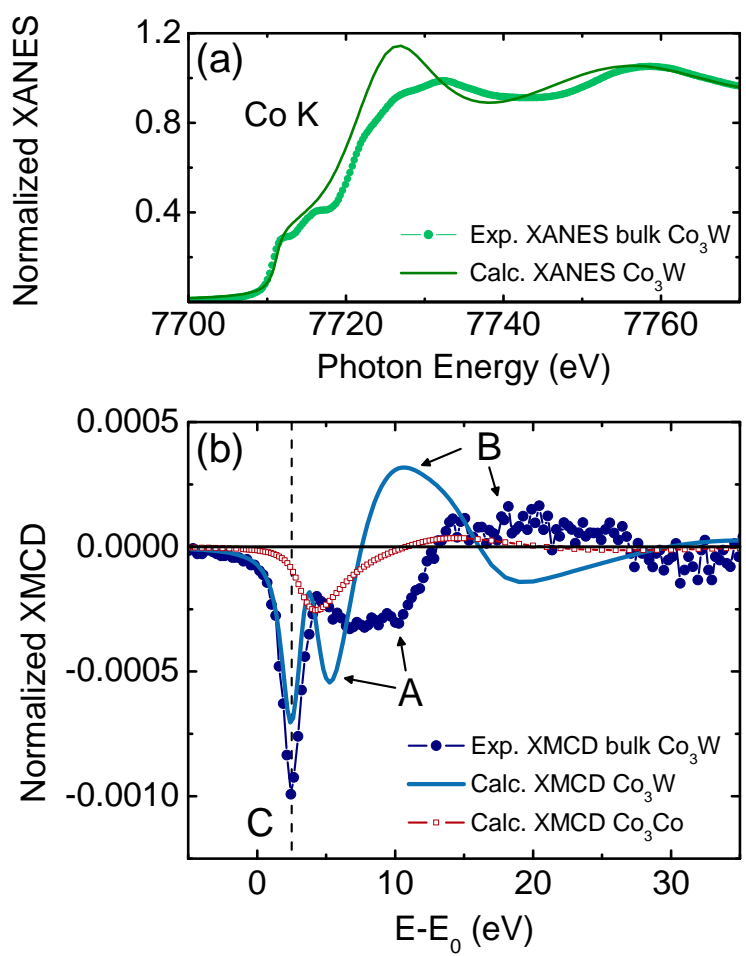

Figure 3.5: (a) Co $K$ edge XANES spectra for the $\mathrm{Co}_{3} \mathrm{~W}$ system calculated with FDMNES (-) and comparison with the experimental one for the alloy (•). (b) Calculated Co $K$ edge XMCD signal for a $\mathrm{Co}_{3} \mathrm{Co}(\square)$ and a $\mathrm{Co}_{3} \mathrm{~W}(-)$ system, and comparison with the experimental one for the $\mathrm{Co}_{3} \mathrm{~W}$ alloy $(\bullet)$.

tween the Co magnetic moment and the $\mathrm{W}$ induced one, as is the general case for intermetallics with less-than-half and more-than-half filled bands [134].

\subsubsection{W $L_{2,3}$ edges}

Calculations of the XANES and XMCD at the W $L_{2,3}$ edges were performed with the same FDMNES code. The intensity and spectral shape of the XMCD calculated signal is very sensitive to the Co magnetic moment introduced in the calculation. In contrast with the Co $K$ edge, the spectral shape of the W XMCD is very sensitive to the approach used within the FDMNES code. In general, a rather satisfactory qualitative agreement is always found, achieving the best with the non muffin-tin approximation, as shown in Fig. 3.6(a). The calculation confirms the antiparallel configuration between the Co magnetic moment and the $\mathrm{W}$ induced one, as described above. It is particularly interesting that the breakdown of Hund's third rule for the W $5 d$ magnetic moments in $\mathrm{Co}_{3} \mathrm{~W}\left(m_{\mathrm{L}} / m_{\mathrm{S}}>0\right)$ is also described in the calculation, as shown in Fig. 


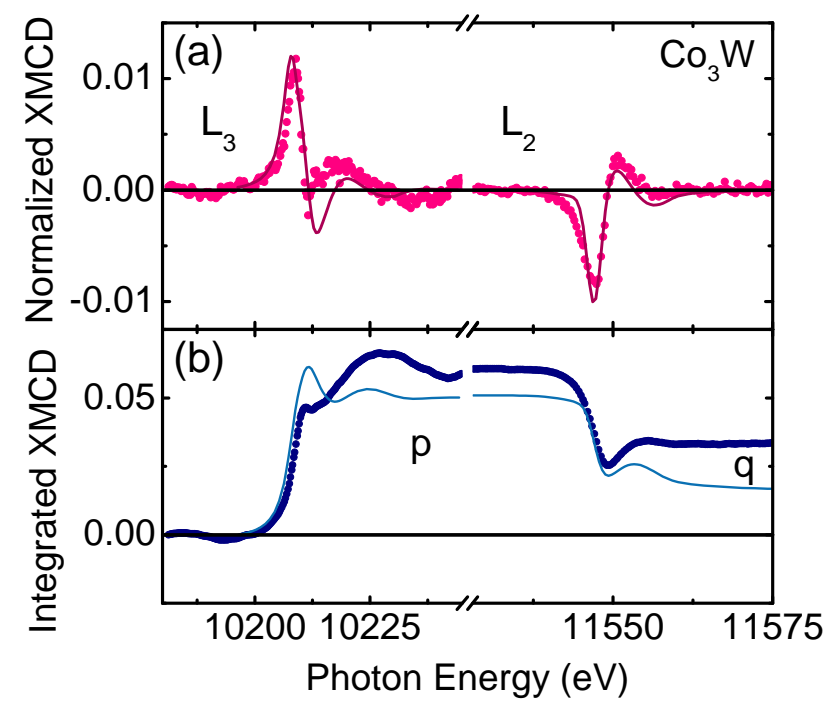

Figure 3.6: (a) Experimental $(\bullet)$ and calculated $(-)$ XMCD signal at the $L_{2,3}$ edges of $\mathrm{W}$ in $\mathrm{Co}_{3} \mathrm{~W}$. (b) Integrals of the experimental $(\bullet)$ and calculated $(-)$ signals in (a).

3.6(b), where the experimental integrals are compared to the calculated ones. The signs of $p$ and $q$ are both positive, as in the experiments, leading to the same quantitative result (see values in table 3.1). Calculations with the multiple scattering formalism with a muffin-tin potential generate extra features which are not observed in the $\mathrm{W} L_{2,3}$ XMCD.

\subsection{Discussion}

The existence of a strong hybridization between the Co $4 p-3 d$ and W $5 d$ orbitals in all the Co-W systems studied has been demonstrated both experimentally and theoretically. Our study of the W $5 d$ band by XANES at the W $L_{2,3}$ edges and DOS calculations evidence electronic transfer from Co $4 p-3 d$ through $\mathrm{W} 5 d$ bands, and its dependence on the $\mathrm{Co} / \mathrm{W}$ ratio in the samples. The main consequence of this hybridization is the existence of an induced magnetic moment in the $\mathrm{W}$ atoms in all the $\mathrm{Co}-\mathrm{W}$ alloy systems. Moreover, the induced spin and orbital moments in $\mathrm{W}$ are found to be parallel aligned $\left(m_{\mathrm{L}} / m_{\mathrm{S}}>0\right)$, which is contrary to the prediction of Hund's third rule for W atoms since it is a less-than half filled $5 d$ band metal.

How hybridization may influence the breakdown of Hund's third rule in metallic compounds was originally proven by Galanakis et al. in a theoretical study on metallic $\mathrm{VAu}_{4}\lfloor 133\rfloor$. They nicely described how the spin-orbit (SO) in Au may influence the orbit in $\mathrm{V}$ atoms through hybridization, provoking the 
reversal of its orbital moment and, therefore, the breakdown of the mentioned rule. Subsequent works on metallic compounds make use of this interpretation to demonstrate that the strong hybridization between the different atoms in the magnetic compounds is the key factor of such a breakdown $[70,120,123]$. Similarly, in our Co-W alloy NPs and in the bulk $\mathrm{Co}_{3} \mathrm{~W}$ alloy, Co spin-orbit coupling can influence the induced W orbital moment causing its reversal.

The effect of the SO coupling in the reversal of the induced orbital moment in $\mathrm{W}$ in $\mathrm{Fe} / \mathrm{W}$ multilayers is described by Wilhelm et al. $\mid 120\rfloor$ using a simple atomic picture based on the competition of interactions between the spin and orbital angular momentum projections in the $3 d$ metal and W. Similarly, in our Co-W alloy systems, the interaction mechanisms may be examined as those then observed on $\mathrm{Fe} / \mathrm{W}$. The dominant interaction in all cases is the spin-spin interatomic exchange coupling between Co and W, $J_{\text {inter }} \cdot S_{z}^{\mathrm{Co}} \cdot S_{z}^{\mathrm{W}}$ which compels the spin in $\mathrm{W}$ to align antiparallel to Co. Then, there is a competition between the intra-atomic SO coupling in $\mathrm{W}, \lambda_{\text {intra }} \cdot S_{z}^{\mathrm{W}} \cdot L_{z}^{\mathrm{W}}$, and the interatomic Co-W SO interaction $\lambda_{\text {inter }} \cdot S_{z}^{\mathrm{Co}} \cdot L_{z}^{\mathrm{W}}$. The breakdown of Hund's third rule occurs since the interatomic Co-W interaction overcomes the intra-atomic SO interaction in $\mathrm{W}$, so that Co spin turns $m_{\mathrm{L}}^{\mathrm{W}}$ to align antiparallel to $m_{\mathrm{S}}^{\mathrm{Co}}$, but parallel to $m_{\mathrm{S}}^{\mathrm{W}}$, resulting in $m_{\mathrm{L}} / m_{\mathrm{S}}>0$ in $\mathrm{W}$.

Further theoretical studies for the layered Fe/W system illustrate the influence of a large crystal field splitting in W, and the hybridization between Fe and $\mathrm{W}$ taking into account the band-filling and geometrical effects [135-137]. The latter are rather determinant, since the interatomic interactions between Fe and $\mathrm{W}$ are mediated by the crystal symmetry and interlayer distances; i.e., an increase of the interatomic Fe-W interaction is observed as the Fe and W interlayer distance decreases, which may be strongly related to the reversal of the orbital moment in W atoms [135].

In fact, recent works on Fe-Zr and Co-Zr amorphous magnetic alloys discuss the strong relationship between the structural properties with the breakdown of Hund's third rule observed in these systems [123]. The dependence of $\mathrm{Co}(\mathrm{Fe})$ spin-orbit coupling with the reduction of the $\mathrm{Co}(\mathrm{Fe})-\mathrm{Zr}$ interatomic distance and the coordination number is determined from first-principles calculations of the $m_{\mathrm{L}} / m_{\mathrm{S}}$ ratio in $\mathrm{Zr}$ in the $\mathrm{Co}(\mathrm{Fe})-\mathrm{Zr}$ alloys, as a function of the lattice parameter, $\alpha$, for various crystallographic structures. A change in sign of $m_{\mathrm{L}} / m_{\mathrm{S}}$ from negative to positive is observed as $\alpha$ decreases. Following an analogous analysis, we may correlate the structural results obtained on the Co-W alloy NPs and on the bulk $\mathrm{Co}_{3} \mathrm{~W}$ alloy from extended X-ray absorption fine structure (EXAFS) in section 2.2.2 with the results of induced moments obtained by XMCD in this chapter. In Fig. 3.7(a), the obtained $m_{\mathrm{L}} / m_{\mathrm{S}}$ in W in all Co-W systems are plotted as a function of the first $\mathrm{Co}-\mathrm{W}$ interatomic 


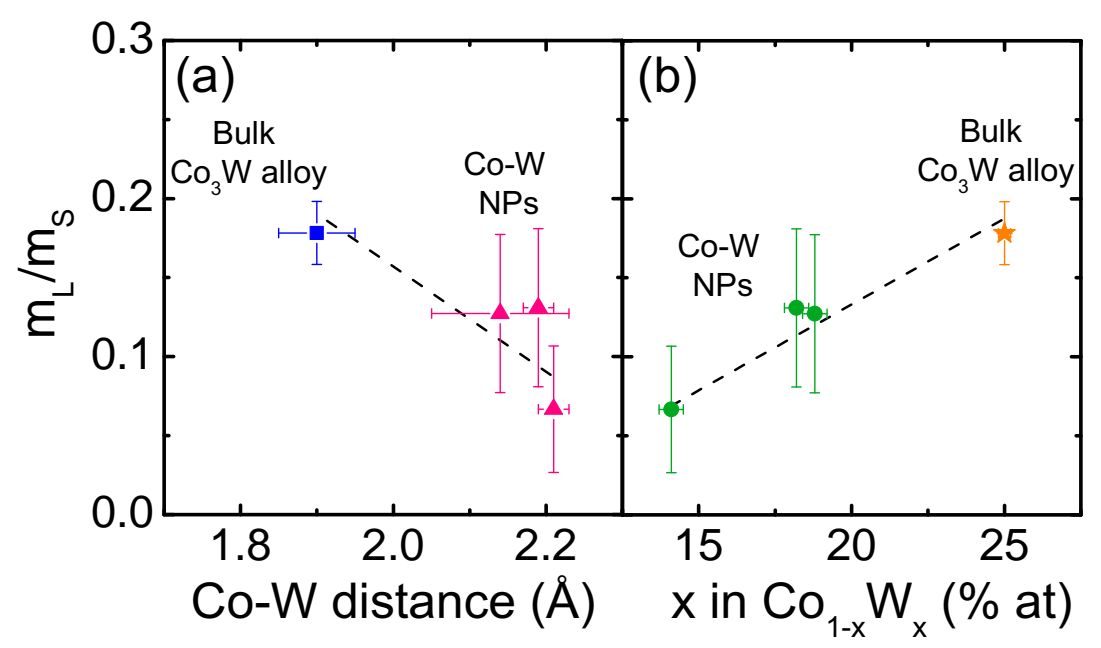

Figure 3.7: $m_{\mathrm{L}} / m_{\mathrm{S}}$ ratio as calculated from the $\mathrm{W} L_{2,3} \mathrm{XMCD}$ signal for the Co-W alloy NPs and for the bulk $\mathrm{Co}_{3} \mathrm{~W}$ alloy, as a function of (a) the first $\mathrm{Co}-\mathrm{W}$ interatomic distance obtained from EXAFS, and (b) the composition of the Co-W alloy in the samples. Dashed lines: guide to the eye.

distance resulting from EXAFS analysis on the same samples. $m_{\mathrm{L}} / m_{\mathrm{S}}$ is found to increase as the Co-W distance shortens. This trend agrees well with the one observed for calculated $m_{\mathrm{L}} / m_{\mathrm{S}}$ versus $\alpha$ in $\mathrm{Zr}$ in the $\mathrm{Co}(\mathrm{Fe})-\mathrm{Zr}$ alloys in Ref. [123]. In the same study, Kapaklis et al. claim that the crystallinity of the materials is determinant in the breakdown of Hund's third rule since $\mathrm{Zr}$ induced moments in crystalline FeZrPt and CoZrPt alloys are found to fulfill the rule. In contrast, our observations of the breakdown of the rule on the polycrystalline bulk $\mathrm{Co}_{3} \mathrm{~W}$, both experimentally and theoretically, demonstrate that the disorder level in the sample is not its main cause; instead, the reduction of the interatomic distances between dissimilar atoms seems to be the crucial point on this very controversial topic.

The Co influence on the orbital moment of $\mathrm{W}$ atoms in the samples may also be examined as a function of the $\mathrm{Co} / \mathrm{W}$ in the alloy. In fact, $m_{\mathrm{L}} / m_{\mathrm{S}}$ is found to scale with the $\mathrm{W}$ concentration in the Co-W alloy, as observed in Fig. 3.7(b). As it was described in section 3.1.2, this may be understood as an increase of the induced orbital component of the $\mathrm{W}$ magnetic moment as the $\mathrm{Co} / \mathrm{W}$ proportion on the alloy decreases. A similar trend was observed for the $m_{\mathrm{L}} / m_{\mathrm{S}}$ induced in Ir in Co-Ir disordered alloys, where Hund's third rule is also not fulfilled $[70\rfloor$. 


\subsection{Conclusion}

In all the $\mathrm{Co}-\mathrm{W}$ alloy samples studied, i.e., Co-W alloy NPs and polycrystalline bulk $\mathrm{Co}_{3} \mathrm{~W}$ alloy, the induced $\mathrm{W}$ orbital moment orients parallel to its spin moment, contrary to the prediction of Hund's third rule for W. This breakdown has been proven by experimental and simulated XANES spectra and XMCD signals at the Co $K$ and $\mathrm{W} L_{2,3}$ edges. Consequently, we demonstrate that $\mathrm{W}$ not only affects the local structure of Co NPs, but also their electronic structure. The Co $3 d-\mathrm{W} 5 d$ hybridization is evidenced and becomes a key factor in the breakdown of this rule in the case of induced moments. Our comparison between XMCD and EXAFS results demonstrate that the influence of the Co$\mathrm{W}$ spin-orbit coupling depends on the average $\mathrm{Co}-\mathrm{W}$ interatomic distance, as consequence of the dependence of the strength of the hybridization. Contrary to the differences found for crystalline and amorphous $\mathrm{Co}(\mathrm{Fe})-\mathrm{Zr}$ alloys in Ref. $\lfloor 123$, we observe the breakdown of Hund's third rule in both amorphous and polycrystalline Co-W systems, which suggests that this behavior is not a general behavior, but depends on the studied material and its local structure. It is worth noting that this is the pioneer observation of the breakdown of this rule on a nanoparticulated system. 


\section{Chapter 4}

\section{Structural and magnetic properties of Co-Pt nanoparticles}

In this section, we describe the structural and magnetic characterization performed on the Pt-capped Co-nanoparticles system. We examine the role of Co-Pt alloying in the origin and enhancement of the magnetic anisotropy in these NPs. In fact, the production of Co-Pt alloys is one of the preferred routes currently used in nanoparticles and thin films to increase their magnetic anisotropy $[22,138]$. This results from the crystallization of Co-Pt alloys in the chemically ordered $L 1_{0}$ phase, which has a very high magnetocrystalline anisotropy for the bulk $[23\rfloor$.

Moreover, many Co-Pt alloy thin films [139-142 $\rfloor$ and assembled nanoparticles systems $[22,30,31,138]$ show perpendicular magnetic anisotropy (PMA): a property which favors the easy axis of magnetization along the film normal direction. Most of these Co-Pt systems are either produced at high temperatures or annealed in order to favor the crystallization of the Co-Pt alloy in the above mentioned ordered $L 1_{0}$ phase. However, the origin of PMA in CoPt alloy systems deposited at room temperature, as in the case of the Co-Pt NPs we describe in this chapter, remains controversial $\lfloor 142\rfloor$.

Previous studies on Co-Pt alloy nanostructured systems have demonstrated the importance of a complete investigation of both the local atomic environment and their long range order to clarify the correlation between crystallographic structure and magnetic anisotropy [142]. Therefore, we have performed a systematic analysis of the structural and magnetic properties of CoPt NPs prepared by sequential sputtering deposition of $\mathrm{Al}_{2} \mathrm{O}_{3}$, Co and Pt on 
a Si substrate. The morphology and structure of the Co-Pt NPs are studied by high-resolution transmission electron microscopy (HRTEM) and extended $\mathrm{X}$-ray absorption fine structure (EXAFS) measurements, with which we prove the presence of Co-Pt alloy in all samples, as it was described for the Co$\mathrm{W}$ alloy NPs. However, contrary to the amorphous structure observed for the Co-W NPs, the Co-Pt alloy NPs consist of a self-organized system of crystalline particles. Their fascinating properties of strong perpendicular magnetic anisotropy are described, as observed by conventional SQUID magnetometry measured with the applied magnetic field parallel and perpendicular to the substrate plane. Their magnetic properties are also explored by anomalous Hall effect (AHE) and X-ray magnetic circular dichroism (XMCD).

This work allows to have a better understanding the origin of PMA in these Co-Pt NPs.

\subsection{Samples studied}

The samples studied in this chapter consist in Pt-capped Co-nanoparticles prepared by Dr. Cyrile Deranlot and Dr. Frederic Petroff at the Unité Mixte de Physique CNRS-Thales ${ }^{1}$ in Palaiseau, France, by sequential sputtering deposition of $\mathrm{Al}_{2} \mathrm{O}_{3}$, Co and $\mathrm{Pt}$ on a $\mathrm{Si}$ substrate. The preparation procedure is the same as that previously described for the Co-W NPs in section 2.1, and illustrated in Fig. 2.1, using $\mathrm{Pt}$ instead of $\mathrm{W}$ in this case. We end up with a multilayer system, following the formula $\mathrm{Al}_{2} \mathrm{O}_{3} /\left(\mathrm{Al}_{2} \mathrm{O}_{3} / \mathrm{Co} / \mathrm{Pt}\right)_{\mathrm{N}} / \mathrm{Al}_{2} \mathrm{O}_{3}$, with $\mathrm{N}$ being the number of repetitions of the $\mathrm{Al}_{2} \mathrm{O}_{3}$, Co and Pt trilayer deposition. The characterization of the Co-Pt NPs that we describe in this chapter has been performed on samples with a fixed $t_{\mathrm{Co}}=0.7 \mathrm{~nm}$, varying $t_{\mathrm{Pt}}=0.6,1.5$, 4.5 and $6.0 \mathrm{~nm}$, and $\mathrm{N}=25$ in all cases. Let us recall that the $\mathrm{Al}_{2} \mathrm{O}_{3}$ layer thickness is $3 \mathrm{~nm}$ in each trilayer, and $5 \mathrm{~nm}$ in the one deposited at the end of the process for protection purposes.

\subsection{Morphological and structural study}

\subsubsection{Microscopy Measurements}

The morphology of the Co-Pt NPs samples has been studied by HRTEM and high angle annular dark field (HAADF) scanning transmission electron microscopy (STEM) measurements, performed by Dr. Luisa Ruiz González and Dr. José María González-Calbet of the Departamento de Química Inorgánica

\footnotetext{
${ }^{1}$ http://www.trt.thalesgroup.com/ump-cnrs-thales/
} 
in the Universidad Complutense de Madrid. ${ }^{2}$ HRTEM images were taken in both cross section and plan view configurations, in a JEOL JEM $3000 \mathrm{~F}$ Field Emission Microscope fitted with an Oxford LINK Electron Dispersive Spectrometry (EDS) analyzer.

Low magnification studies in cross section configuration for all four samples confirmed the presence of 25 layers in agreement to the nominal layer preparation. HRTEM images, shown in Fig. 4.1, and EDS analysis indicate that light gray layers correspond to the amorphous alumina and darker layers to the Co and Pt metals, similarly to the case of Co-W NPs described in section 2.2. In contrast to the Co-W NPs case, crystallinity in the Co-Pt NPs is observed in all four samples studied by HRTEM. Each metallic layer is about $2.3,2.5,5.5$ and $6.7 \mathrm{~nm}$ thick, for the samples with $t_{\mathrm{Pt}}=0.6,1.5,4.5$ and 6.0 $\mathrm{nm}$, respectively.

In the samples with the lowest amount of $\mathrm{Pt}\left(t_{\mathrm{Pt}}=0.6\right.$ and $\left.1.5 \mathrm{~nm}\right)$ there

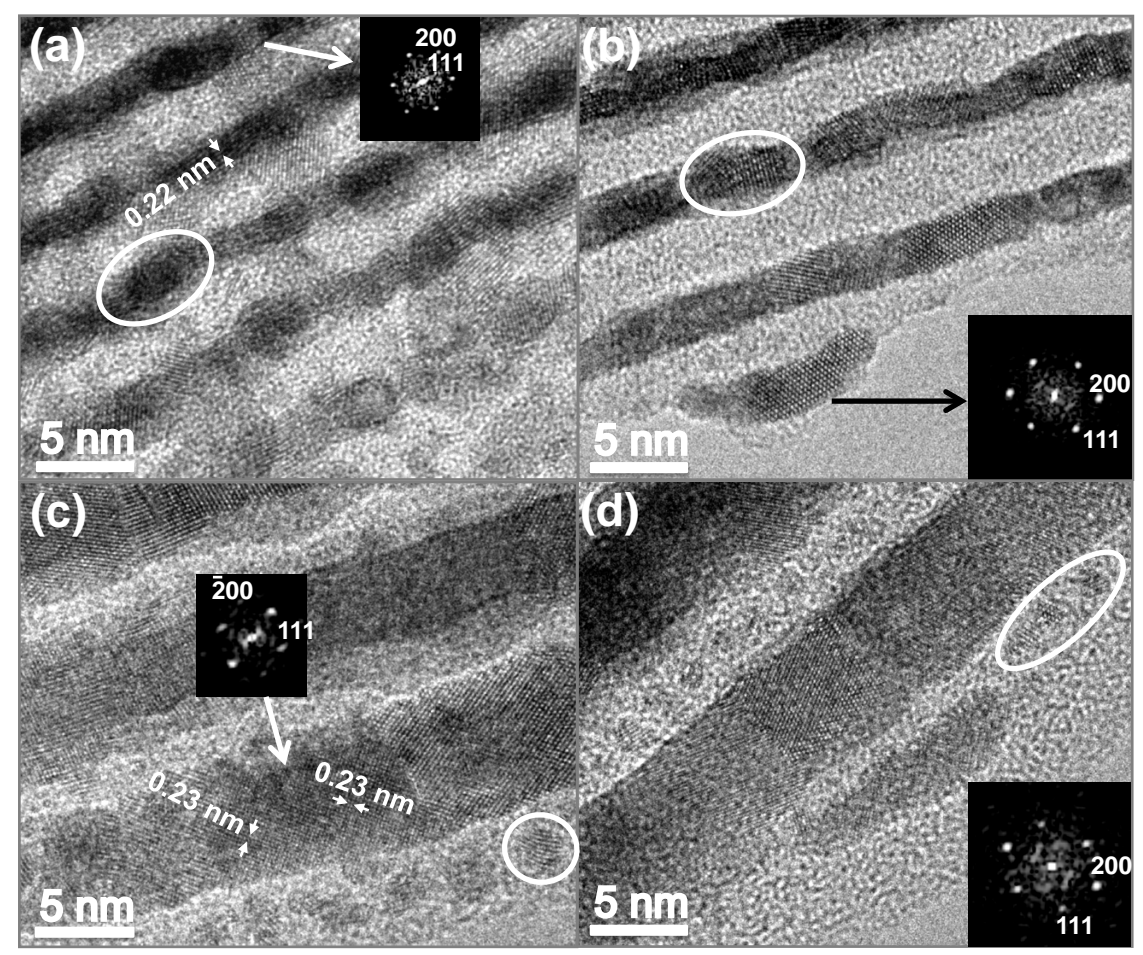

Figure 4.1: HRTEM images in cross section configuration for samples from with $t_{\mathrm{Co}}=0.7 \mathrm{~nm}$ and (a) $t_{\mathrm{Pt}}=0.6 \mathrm{~nm}$, (b) $t_{\mathrm{Pt}}=1.5 \mathrm{~nm}$, (c) $t_{\mathrm{Pt}}=4.5 \mathrm{~nm}$ and (d) $t_{\mathrm{Pt}}=6.0 \mathrm{~nm}$. Fourier transforms (FFTs) at selected zones of the images are shown. A Co-Pt particle is highlighted in (a) and (b). Pt NPs are marked in (c) and (d).

\footnotetext{
${ }^{2}$ http://www.cnme.es/
} 
are some signs of particulated morphology, as in the region surrounded by a white ellipse in Figs. 4.1(a) and 4.1(b). Some darker shadows inside the layers indicate the presence of $\mathrm{Co}$, located at the center of the layer, suggesting that the capping $\mathrm{Pt}$ tends to surround the Co particle. For the other two samples $\left(t_{\mathrm{Pt}}=4.5\right.$ and $\left.6.0 \mathrm{~nm}\right)$, however, the Co-Pt nanoparticles are not easy to be identified from the images obtained in cross section configuration.

Fast Fourier transform (FFT) performed on selected areas of the images shows spots that are well indexed with the interplanar distances of a $f c c$ cell in all cases (see insets in Fig. 4.1). In Figs. 4.1(a) and 4.1(c) the periodicity found in the selected zone of the image is $0.22-0.23 \mathrm{~nm}$, which is characteristic of the (llll) planes of a $f c c$ Pt structure. In Fig. 4.1(c) and 4.1(d) some small crystalline clusters in the alumina region of the samples are observed (see circled particles in the figures), which have been identified as Pt clusters with $f c c$ structure, ranging between 2 and $3 \mathrm{~nm}$ in diameter.

Improved contrast to better identify the Co-Pt particles is obtained by plan view STEM images, such as the one shown for the sample with $t_{\mathrm{Co}}=0.7 \mathrm{~nm}$ and $t_{\mathrm{Pt}}=0.6 \mathrm{~nm}$ in Fig. $4.2(\mathrm{a})$. In this image, brighter parts correspond to Co-Pt NPs, and darker parts to $\mathrm{Al}_{2} \mathrm{O}_{3}$. The image obtained is comparable to those of similar systems of metallic nanoparticles embedded in insulator matrices [93], and the shape of the particles is similar to that of Co-nanoparticles embedded in alumina [94], and to the one found for the Co-W NPs (see section 2.2.1).

The average diameter of the Co-Pt NPs from the image in Fig. 4.2(a) was

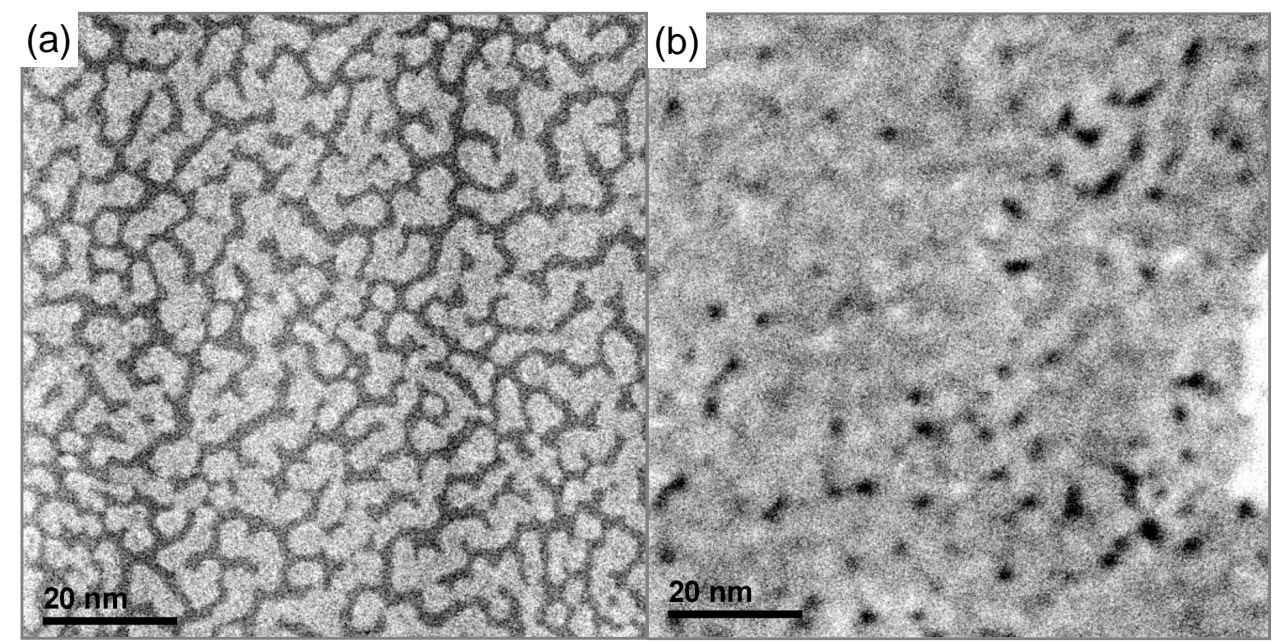

Figure 4.2: $\quad$ STEM images in plan view configuration for the samples with $t_{\mathrm{Co}}=0.7$ $\mathrm{nm}$, and (a) $t_{\mathrm{Pt}}=0.6 \mathrm{~nm}$ and (b) $t_{\mathrm{Pt}}=1.5 \mathrm{~nm}$. 
4.1(2) nm. STEM images for the other three samples (those with $t_{\mathrm{Pt}}=1.5$, 4.5 and $6.0 \mathrm{~nm}$ ) did not allowed identification of the Co-Pt particles since they revealed an almost uniform Pt capping, as shown in Fig. 4.2(b) for the sample with $t_{\mathrm{Pt}}=1.5 \mathrm{~nm}$.

\subsubsection{XANES and EXAFS measurements}

X-ray absorption (XAS) and EXAFS spectra for the Co-Pt NPs samples with $t_{\mathrm{Co}}=0.7 \mathrm{~nm}$ and $t_{\mathrm{Pt}}=0.6,1.5,4.5$ and $6.0 \mathrm{~nm}$ were measured at the Co $K$ $(7709 \mathrm{eV})$ and $\mathrm{Pt} L_{3}(11564 \mathrm{eV})$ edges using a double-crystal Si(111) monocromator in the BM29 beamline at the European Synchrotron Radiation Facility (ESRF). Measurements were performed at $300 \mathrm{~K}$ in fluorescence detection mode with a 13-element Ge solid state detector with digital signal processing for fluorescence XAS, high energy resolution and high count rate. Reference samples were also measured at different edges: Co particles embedded in alumina matrix (referred in the following as "bare Co NPs") and the Co metallic foil measured at the Co $K$ edge; and Pt metallic foil measured at the $\mathrm{Pt} L_{3}$ edge. For each edge measured on each sample, four spectra were taken in order to have enough statistics in data treatment, following a $4 \mathrm{~s} /$ point count.

The X-ray absorption spectra were analyzed following standard procedures. A preliminary reduction of the EXAFS raw data involving background removal of the absorption data $\mu(\mathrm{E})$, conversion of $\mu(\mathrm{E})$ to $\chi(\mathrm{k})$, normalization and weighting scheme were performed with AUTOBK and ATHENA, programs from the simulation package IFEFFIT 96 . EXAFS data analysis and fitting were performed on all reference and samples at their respective edges. All spectra were fitted using the ARTEMIS program from the same IFEFFIT package using models based on crystallographic information found in the ICSD $^{3}$ database. Atomic clusters used to generate the scattering paths for fitting were generated with ATOMS, a tool from the same simulation package $\lfloor 97\rfloor$.

\section{Co $K$ edge}

Normalized X-ray absorption near edge structure (XANES) spectra at the Co $K$ edge for Co-Pt NPs with $t_{\mathrm{Co}}=0.7 \mathrm{~nm}$ and $t_{\mathrm{Pt}}=0.6,1.5,4.5$ and 6.0 $\mathrm{nm}$ are shown in Fig. 4.3, along with the Co foil XANES, plotted for reference. Changes in the intensity and shape of the pre-edge peak and the white line (denoted as peak I and peak II, respectively, in Fig. 4.3) are appreciable and reflect changes in the electronic structure of the Co (elec-

\footnotetext{
${ }^{3}$ http://icsd.iqfr.csic.es/icsd/
} 


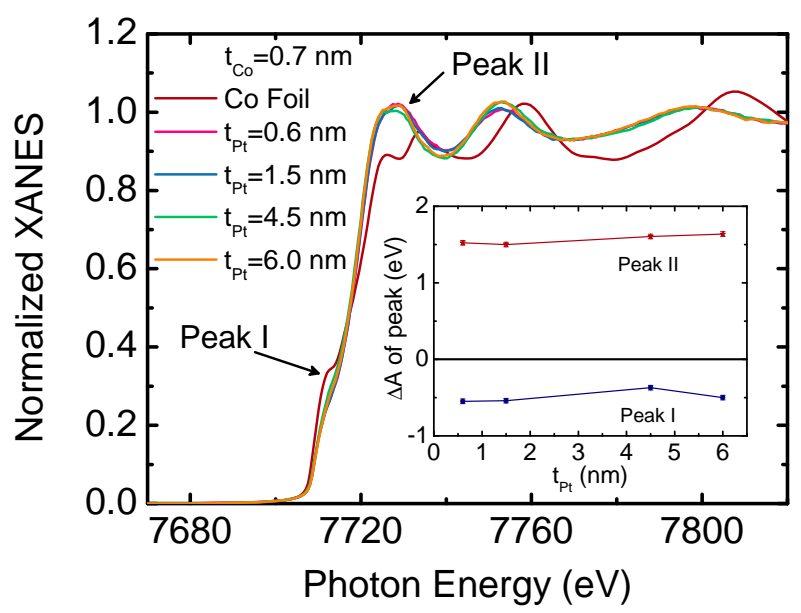

Figure 4.3: XANES at the Co $K$ edge for the Co-Pt NPs and comparison with that of a Co foil for reference. Inset: Variation of the areas of peak I and peak II in the figure as a function of $t_{\mathrm{Pt}}$.

tron configuration $[\mathrm{Ar}] 3 d^{7} 4 s^{2}$ ) and $\mathrm{Pt}$ (electron configuration $[\mathrm{Xe}] 4 f^{14} 5 d^{9} 6 s^{1}$ ) atoms. Analogous to the analysis of such variation described for the Co $K$ edge XANES in the Co-W NPs, we calculate the difference of the peaks areas $\left(\Delta \mathrm{A}=\int_{E_{i}}^{E_{f}}\left(\mu_{\text {sample }}-\mu_{\text {Co-foil }}\right) d E\right)$. The inset of Fig. 4.3 represents the $\Delta \mathrm{A}$ as a function of the Pt content in the samples. The integration limits were 7706.1-7717.6 eV for peak I, and 7717.6-7735.1 eV for peak II.

Let us recall that the Co $K$ edge white line reflects the $4 p$ empty states of Co near the Fermi energy. Variation of peak I mainly determines changes of the $3 d$ character of unoccupied states, which is influenced by Co $4 p-3 d$ hybridization. The reduction of the intensity of peak I in the Co-Pt NPs with respect to that of the Co foil, also observed for the in Co-W NPs case, reveals a delocalization of the Co $3 d$ empty states, and is a proof of Co $4 p-3 d-\mathrm{Pt}$ $5 d$ hybridization.

The intensity of peak II depends on both the number of available $4 p$ states, and the structure of the system. It is evident that the intensity of this peak II is higher and its shape different from that of the Co foil. Indeed, it looks closer to those recorded for Co-Pt alloys in Ref. [143, which may be an indication of presence of such kind of alloys in our Co-Pt particles. In any case, it is clear that the $4 p$ empty states in the Co-Pt NPs samples increase, compared to those of the bulk Co.

Both peak I and peak II are practically constant along the Co-Pt samples series (see inset of Fig. 4.3(a)), reflecting that Co $3 d-\mathrm{Pt} 5 d$ hybridization is not affected by such variations in the amount of $\mathrm{Pt}$ for a fixed Co content. 

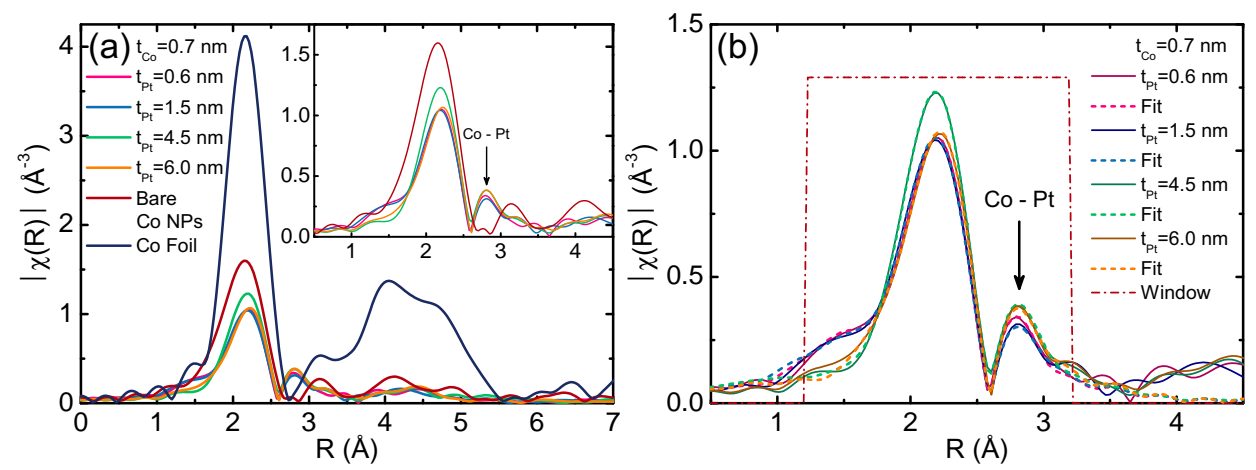

Figure 4.4: (a) Fourier transform of EXAFS signal at the Co $K$ edge on the Co-Pt samples. The measurements on the Co foil and bare Co nanoparticles with $t_{\mathrm{Co}}=0.7$ $\mathrm{nm}$ in are shown for comparison. Inset: zoom of the first coordination shell in the particulate systems. (b) Best fits to the first coordination shell in (a) for the Co-Pt nanoparticles.

Similarly to the Co-W NPs case, this fact strongly suggests that the alloy composition in the Co-Pt NPs samples is similar for these four samples studied, a result that will be confirmed by EXAFS (later in this section).

The structural analysis of the Co-Pt NPs that can be extracted from the $\chi(\mathrm{k})$ EXAFS signal is better studied by its Fourier transform (FT), shown in Fig. 4.4(a), performed on a k-range from 2.4 to $10.6 \AA^{-1}$ using a $\mathrm{k}^{2}$ weight and a kaiser-bessel window function. This Co $K$ edge $R$-space data show peaks which correspond to different atomic shells surrounding the Co atoms in the samples. We have plotted the FT EXAFS of Co-Pt NPs samples along with the Co foil and bare Co NPs for comparison. As described in section 2.2 .2 , the Co environment of the latter two samples is very similar, which is revealed from the the same position and shape of the peaks, not only for the first coordination shell, but also for outer shells. However, the peaks for outer coordination shells are different in the Pt-capped Co nanoparticles. The appearance of a peak characteristic of a $\mathrm{Co}-\mathrm{Pt}$ distance is identified in all four samples, and is marked in the inset of Fig. 4.4(a). The intensities of all peaks of the FT EXAFS are larger in the Co foil than in the particulate systems, as it is expected from the reduction in the mean Co coordination due to the size reduction.

From a qualitative inspection of the peaks shape and position of the FT EXAFS at the Co $K$ edge for the four Co-Pt NPs, it is noticeable that the Co neighborhood is very similar in all four samples. This was confirmed by the fitting of EXAFS signal, performed on the $R$-space in a range from 1.2 to $3.2 \AA$ using a kaiser-bessel window function, so that it covered the Co first coordination shell (see Fig. 4.4(b)). Similarly to the fitting procedure 
performed for the Co-W NPs, different models were tested in order to fit the Co $K$ edge $R$-space data. The best fits were achieved with combinations of two Co-Co distances used when fitting the bare Co NPs: a short Co-Co path at around $2.0 \AA$ and a Co-Co belonging to the $f c c$ Co metal at $2.51 \AA$; and two distances belonging to a CoPt alloy: a Co-Co path at $2.65 \AA$ and a Co-Pt at $2.70 \AA .{ }^{4}$

The structural parameters for fitting the $R$-space EXAFS at the Co $K$ edge of each Co-Pt NPs sample were the average coordination $(N)$, interatomic distance $(R)$, and the Debye-Waller factor $\left(\sigma^{2}\right)$ for each path. The amplitude reduction factor $S_{0}^{2}$ was set to that obtained for the Co foil fit, and the shift in the threshold energy $\Delta E_{0}$ was fit for each sample. A total of 10 fitting parameters were used for an iterative procedure, which consistently included the fitting of both Co $K$ and Pt $L_{3}$ edges (see section 4.2.2). Results for the Co $K$ edge EXAFS fits for the Co-Pt NPs are shown in Fig. 4.4(b) and the values obtained for each sample are listed in table 4.1.

The EXAFS results of distances and coordinations in our Co-Pt NPs differ from those of similar Co-core Pt-shell NPs of comparable sizes [144], demonstrating that the structure of our particles is different from that of a simple Co-core Pt-shell. However, there is a presence of a Co core in the Co-Pt NPs samples since the first two distances Co-Co in table 4.1 (numbered 1. CoCo and 2. Co-Co) are in agreement to those found in the EXAFS fitting of the bare Co NPs, though with a smaller Co-Co coordination due to the size reduction of the Co core. For the samples with $t_{\mathrm{Pt}}=4.5$ and $6.0 \mathrm{~nm}$, the introduction of the 1. Co-Co path had no effect in the fit, therefore it was excluded for the model used for them.

The distances numbered 3. Co-Co and 4. Co-Pt have been identified to belong to a $\mathrm{Co}_{x} \mathrm{Pt}_{1-x}$ alloy with composition close to that with $x=0.5$, by comparison to the Co $K$ edge EXAFS results in Co-Pt films with that composition $\lfloor 142\rfloor$. Such distances, as well as the coordination and Debye-Waller factors for each path, are almost constant along the series, which indicates that the Co-Pt alloy in the samples has the same or very close composition.

\section{Pt $L_{3}$ edge}

Normalized XANES spectra at the Pt $L_{3}$ edge for the Co-Pt NPs samples are shown in Fig. 4.5, along with that of a $\mathrm{Pt}$ foil at the same edge for comparison. Similar to the case of $\mathrm{W}$, being both $5 d$ metals, the $\mathrm{Pt} L_{3}$ absorption edge appears for transitions from $2 p$ to both $5 d_{3 / 2}$ and $5 d_{5 / 2}$ bands. In general, the white line feature of the $\mathrm{Pt} L_{3}$ in the Co-Pt NPs in Fig. 4.5 is reduced with

\footnotetext{
${ }^{4}$ http://icsd.iqfr.csic.es/icsd/
} 


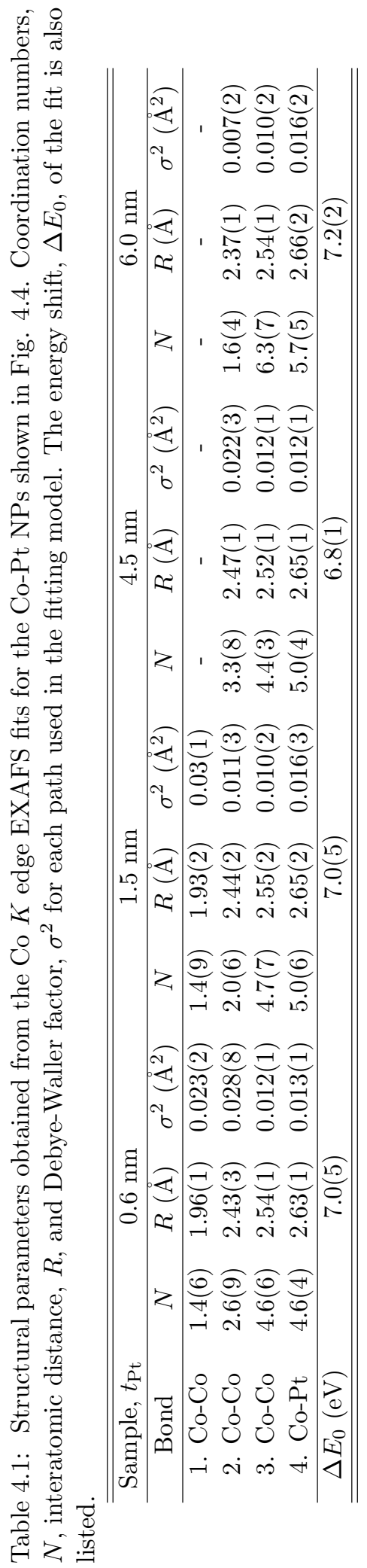




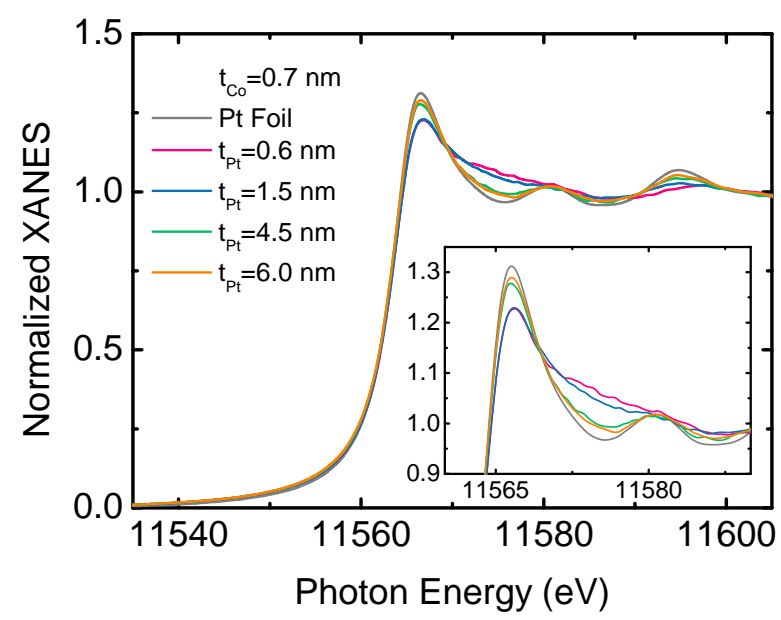

Figure 4.5: XANES spectra at the Pt $L_{3}$ edge for the Co-Pt NPs samples, along with the the absorption of the $\mathrm{Pt}$ foil plotted for reference. Inset: details of the variation of the white line intensity.

respect to that of the $\mathrm{Pt}$ foil as the amount of $\mathrm{Pt}$ in the sample decreases.

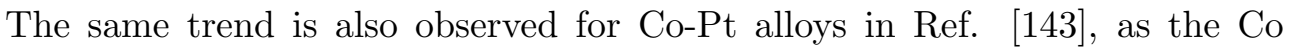
concentration in the alloy increases. A decrease in the $\mathrm{Pt} L_{3}$ edge intensity from one sample to the other shows a decrease in the number of $\mathrm{Pt} 5 d$ available empty states. Such a decrease may be understood as an electron transfer from Co towards Pt, due to hybridization between the Pt $5 d$ and Co $4 p-3 d$ electronic states.

It is evident that those samples with high amount of $\mathrm{Pt}$, namely $t_{\mathrm{Pt}}=4.5$ and $6.0 \mathrm{~nm}$, on one hand, have a spectrum very close in shape and intensity to that of the Pt foil, showing a higher amount of metallic Pt in these two samples. On the other hand, the XANES of those samples with low amount of $\mathrm{Pt}$, that is $t_{\mathrm{Pt}}=0.6$ and $1.5 \mathrm{~nm}$, differ from that of the $\mathrm{Pt}$ foil, showing a broader and less intense white line, which is an indicative of different electronic structure and $\mathrm{Pt}$ environment in these last two samples. Later in this section we will demonstrate by EXAFS analysis performed in these samples how the Pt environment differs, being very similar to the metallic $\mathrm{Pt}$ in the first two mentioned samples $\left(t_{\mathrm{Pt}}=4.5\right.$ and $\left.6.0 \mathrm{~nm}\right)$.

The Fourier transformed EXAFS signals at the Pt $L_{3}$ of the Co-Pt NPs samples, along with that of the $\mathrm{Pt}$ foil used for reference, are shown in Fig. 4.6(a). The k-range for the FT goes from 2.7 to $8.5 \AA^{-1}$ using a $\mathrm{k}^{2}$ weight and a kaiser-bessel window function. A qualitative inspection of the peaks shows, again, that the $\mathrm{Pt}$ environment in the samples with $t_{\mathrm{Pt}}=4.5$ and $6.0 \mathrm{~nm}$ is very similar to that of the metallic Pt. 

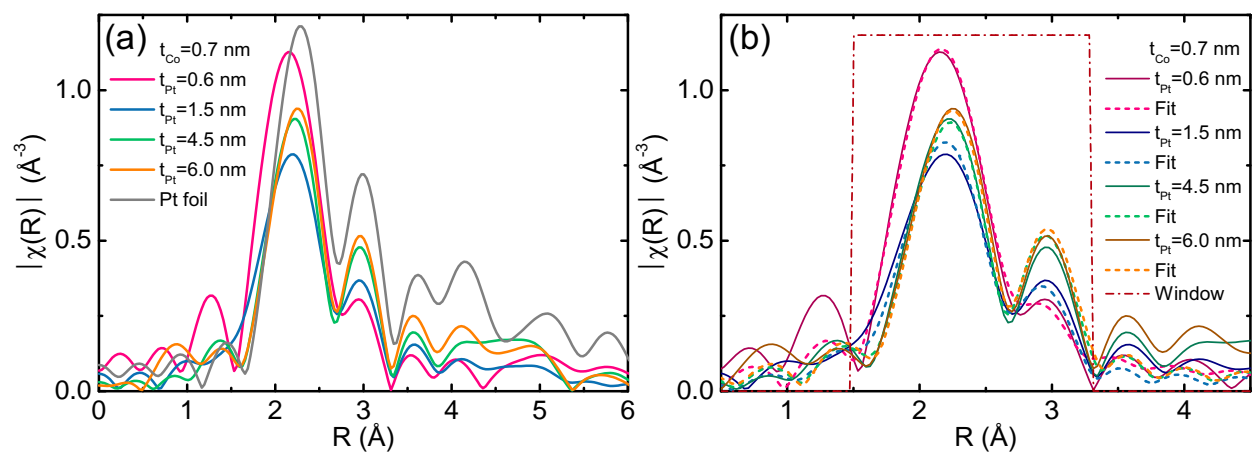

Figure 4.6: (a) Fourier transform of EXAFS signal at the Pt $L_{3}$ edge on the CoPt NPs studied and comparison with that of the Pt foil. (b) Best fits to the first coordination shell of the Co - Pt NPs samples in (a).

The $\mathrm{Pt} L_{3}$ EXAFS fitting was performed on the $R$-space in a $R$-range from 1.5 to $3.3 \AA$, using a kaiser-bessel window function. $\mathrm{S}_{0}^{2}$ was set to that value obtained for the Pt foil EXAFS fit, and $\Delta E_{0}$ was fit for each sample. The Pt $L_{3}$ EXAFS fitting model for the Co-Pt NPs included a combination of Co-Pt distances from a Co-Pt alloy, and a Pt-Pt distance from the metallic Pt. ${ }^{5}$ The fitting parameters were seven, in total, in an iterative procedure including the fitting of both Co $K$ and $\mathrm{Pt} L_{3}$ edges, as it was previously described.

The EXAFS fitting results for the Co-Pt NPs samples are plotted in Fig. 4.6(b) and the values listed in table 4.2. The Pt-Co distance of $2.62 \AA$ found for the samples with $t_{\mathrm{Pt}}=0.6$ and $1.5 \mathrm{~nm}$ are well in agreement to the results found for the same samples at the Co $K$ edge EXAFS analysis (see table 4.1), which confirms the presence of a Co-Pt alloy in the samples.

The sample with the least amount of $\mathrm{Pt}, t_{\mathrm{Pt}}=0.6 \mathrm{~nm}$, has a $\mathrm{Pt}$ environment quite different from the other three samples. The Pt-Pt distance is in this case a bit shorter $(2.65 \AA)$ than those found for the others $(\sim 2.73 \AA)$, which might indicate that $\mathrm{Pt}$ is not in its metallic form. Such a short PtPt distance has been detected in electrochemically deposited $f c c$ Co-Pt alloys 145」. Then, it is very likely that most of the deposited Pt in this sample may be alloyed with the Co atoms.

The EXAFS signals of the samples with the highest amount of Pt, namely, $t_{\mathrm{Pt}}=4.5$ and $6.0 \mathrm{~nm}$, were fitted only with a Pt-Pt model since introducing a Co-Pt distance had no effect in their fit. From the fitting results in these two samples, we found the same structure around the Pt atoms, as it was observed from the $\mathrm{Pt} L_{3}$ XANES, being very close to that of the Pt foil. In fact, the Pt-Pt coordination being close to 12, and interatomic distances of

\footnotetext{
${ }^{5}$ http://icsd.iqfr.csic.es/icsd/
} 
Table 4.2: Structural parameters obtained from the Pt $L_{3}$ edge EXAFS fits for the Co-Pt NPs shown in Fig. 4.6(b). Coordination numbers, $N$, interatomic distance, $R$, and Debye-Waller factor, $\sigma^{2}$ for both paths used in the fitting model. The energy shift, $\Delta E_{0}$, of the fit is also listed.

\begin{tabular}{cccccccc}
\hline \hline & \multicolumn{5}{c}{ Pt-Co } & \multicolumn{3}{c}{ Pt-Pt } & \\
\cline { 2 - 7 } Sample, $t_{\mathrm{Pt}}$ & $N$ & $R(\AA)$ & $\sigma^{2}\left(\AA^{2}\right)$ & $N$ & $R(\AA)$ & $\sigma^{2}\left(\AA^{2}\right)$ & $\Delta E_{0}(\mathrm{eV})$ \\
\hline $0.6 \mathrm{~nm}$ & $4.6(9)$ & $2.62(3)$ & $0.008(3)$ & $4.7(9)$ & $2.65(3)$ & $0.004(4)$ & $3.2(9)$ \\
$1.5 \mathrm{~nm}$ & $2.4(5)$ & $2.62(2)$ & $0.012(2)$ & $9.3(5)$ & $2.73(2)$ & $0.008(4)$ & $4.7(8)$ \\
$4.5 \mathrm{~nm}$ & - & - & - & $11.8(5)$ & $2.73(1)$ & $0.006(1)$ & $6.7(9)$ \\
$6.0 \mathrm{~nm}$ & - & - & - & $11.8(5)$ & $2.74(1)$ & $0.006(1)$ & $7.2(8)$ \\
\hline \hline
\end{tabular}

$2.73-2.74 \AA$ are close to those obtained for the Pt foil used as a reference with fcc structure.

Similar Co-Co, Co-Pt and Pt-Pt distances to those found for our Co-Pt NPs have been reported for Co-clusters of similar sizes $(\sim 3 \mathrm{~nm})$ embedded in a Pt matrix, where diffusion of the atoms at the surface cluster takes place, resulting in a Pt-Co alloyed interface of one or two monolayers [146]. Analogous processes may be present in our Co-Pt NPs. However, the coordination numbers we have obtained for the Co-Pt and Pt-Pt environments are much higher than those listed in Ref. [146], which may indicate that the Co-Pt alloy interface in our Co-Pt NPs extends to more layers at the Co-core surface.

Summarizing, the morphological and structural characterization of the CoPt NPs reveal the presence of a Co-Pt alloy with a composition very similar in the four samples studied. Its composition is close to a $\mathrm{Co}_{0.5} \mathrm{Pt}_{0.5}$, according to the Co and $\mathrm{Pt}$ coordination and to the Co-Co, Co-Pt and Pt-Pt distances found by EXAFS analysis of the samples. In addition to this alloy, a remnant of the original Co cluster formed on the alumina matrix was identified from the EXAFS fitting at the Co $K$ edge. Thus, the $\mathrm{Co}_{0.5} \mathrm{Pt}_{0.5}$ alloy is probably surrounding this Co rich core. In the samples with $t_{\mathrm{Pt}}=1.5,4.5$ and 6.0 $\mathrm{nm}$ there is an excess of metallic Pt that crystallizes as an $f c c$ structure, in agreement to what was observed by HRTEM for these samples. The presence of small $\mathrm{Pt}$ clusters in the $\mathrm{Al}_{2} \mathrm{O}_{3}$ matrix is also observed from the HRTEM images of the latter two samples. The structure of such Pt clusters is that of metallic $f c c \mathrm{Pt}$, which contributes to the signal of metallic Pt identified in the $\mathrm{Pt} L_{3}$ edge EXAFS. 


\subsection{Magnetic study}

\subsubsection{SQUID-based magnetometry}

Magnetization measurements were performed with a SQUID magnetometer equipped with the high resolution (RSO) option. Both magnetization as a function of the applied field, $M(H)$, and as a function of temperature, $M(T)$, were measured in two configurations: (1) with the field applied parallel, and (2) perpendicular to the substrate plane.

The dc susceptibility measurements curves measured with a bias field of $H_{\mathrm{dc}}=200$ Oe, in both configurations, for the samples series with $t_{\mathrm{Co}}=0.7$ $\mathrm{nm}, t_{\mathrm{Pt}}=1.5,4.5$ and $6 \mathrm{~nm}$, and $N=25$ are plotted in the left panel of Fig. 4.7. The shape of the curves is very different to that found for the bare Co NPs of the same $t_{\mathrm{Co}}=0.7 \mathrm{~nm}$, which show the typical ZFC-FC curve of a superparamagnetic system with a blocking temperature $T_{B}=27 \mathrm{~K}\lfloor 18\rfloor$. In the case of the Co-Pt NPs, the ZFC and FC curves measured in the parallel direction overlap even for fields as high as $50 \mathrm{kOe}$, showing a maximum at the freezing temperature $T_{f}=365,340$ and $296 \mathrm{~K}$ for the samples with $t_{\mathrm{Pt}}=1.5$, 4.5 and $6 \mathrm{~nm}$, respectively. The same cusp is observed in ac susceptibility data, measured with a 4 Oe exciting field. Between 0.1 and $50 \mathrm{~Hz}$, these data show no dependence on frequency, therefore, the analysis of the magnetic anisotropy performed in section 2.3 from ac susceptibility measurements was not possible for these Co-Pt NPs.

In all samples, increasing of the the external applied field $H_{\mathrm{dc}}$ shifts $T_{f}$ to lower temperatures till the maximum is flattened and all the particles become fully polarized along $H_{\mathrm{dc}}$, as observed in the right panel of Fig. 4.7. The dependence of $T_{f}$ with $H_{\mathrm{dc}}$ is a monotonously decreasing function $M\left(T_{f}, H\right)$, as shown in Fig. 4.7 .

In contrast, the ZFC-FC curves measured in the perpendicular configuration show no overlapping as the temperature increases till it reaches $T_{f}$. For the ZFC curve, an increase of the magnetization with temperature is observed until $T_{1} \approx 140 \mathrm{~K}$ for the three samples in the series, which corresponds to the first shoulder observed in the curves in Fig. 4.7(a) to 4.7(c). Above $T_{1}$, the particles follow a collective ferromagnetic behavior up to $T_{f}$, where both ZFC and FC curves decrease again, following a Curie-Weiss law.

The $M(H)$ were measured for all samples with both configurations at several temperatures. Fig. 4.8 shows the data recorded at $5 \mathrm{~K}$. At low temperatures the hysteresis curves measured in the perpendicular direction are rather square, which indicates the presence of a ferromagnetic hard component, with a quite high coercive field of $H_{\mathrm{C}}=4,3$ and $1.9 \mathrm{kOe}$ for the samples 

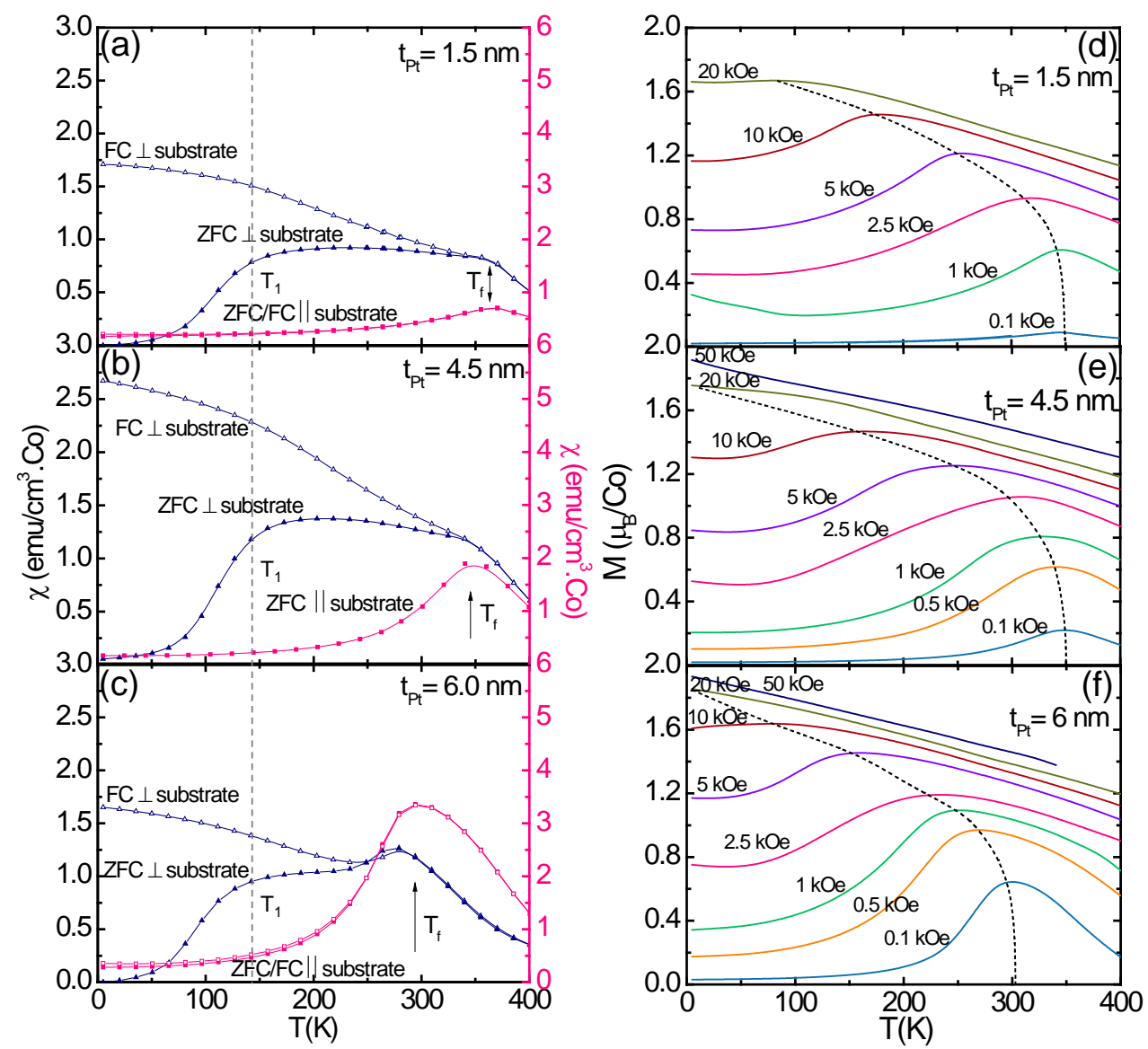

Figure 4.7: (left panel) DC susceptibility curves measured under an applied field of $H_{\mathrm{dc}}=200$ Oe perpendicular and parallel to the substrate plane, and (right panel) $M(T)$ at various fields applied in the parallel direction, $(H$, indicated beside each curve) for samples with $t_{\mathrm{Co}}=0.7, N=25$, and $t_{\mathrm{Pt}}=1.5 \mathrm{~nm}$ in (a) and (d), $t_{\mathrm{Pt}}=4.5$ $\mathrm{nm}$ in (b) and (e), and $t_{\mathrm{Pt}}=6 \mathrm{~nm}$ in (c) and (f) . (- -) $M\left(T_{f}, H\right)$, superparamagneticcorrelated phase separation line.

with $t_{\mathrm{Pt}}=1.5,4.5$ and $6 \mathrm{~nm}$, respectively, at $T=5 \mathrm{~K}$. Such a ferromagnetic behavior implies that the Co-Pt particles are strongly coupled.

In addition to the main reversal of the magnetization close to $H_{\mathrm{C}}$, the hysteresis curves measured at low temperatures in the perpendicular direction have a slight step near $H=0$, which indicates that there is a magnetically soft component present in the sample.

The coercive field measured in the perpendicular direction decreases with temperature till it disappears at about $200 \mathrm{~K}$ for all samples in the series, as shown in Fig. 4.9. $H_{\mathrm{C}}$ also decreases as $t_{\mathrm{Pt}}$ in the sample increases, except for some temperatures near $100 \mathrm{~K}$, where $H_{\mathrm{C}}$ measured for the sample with 


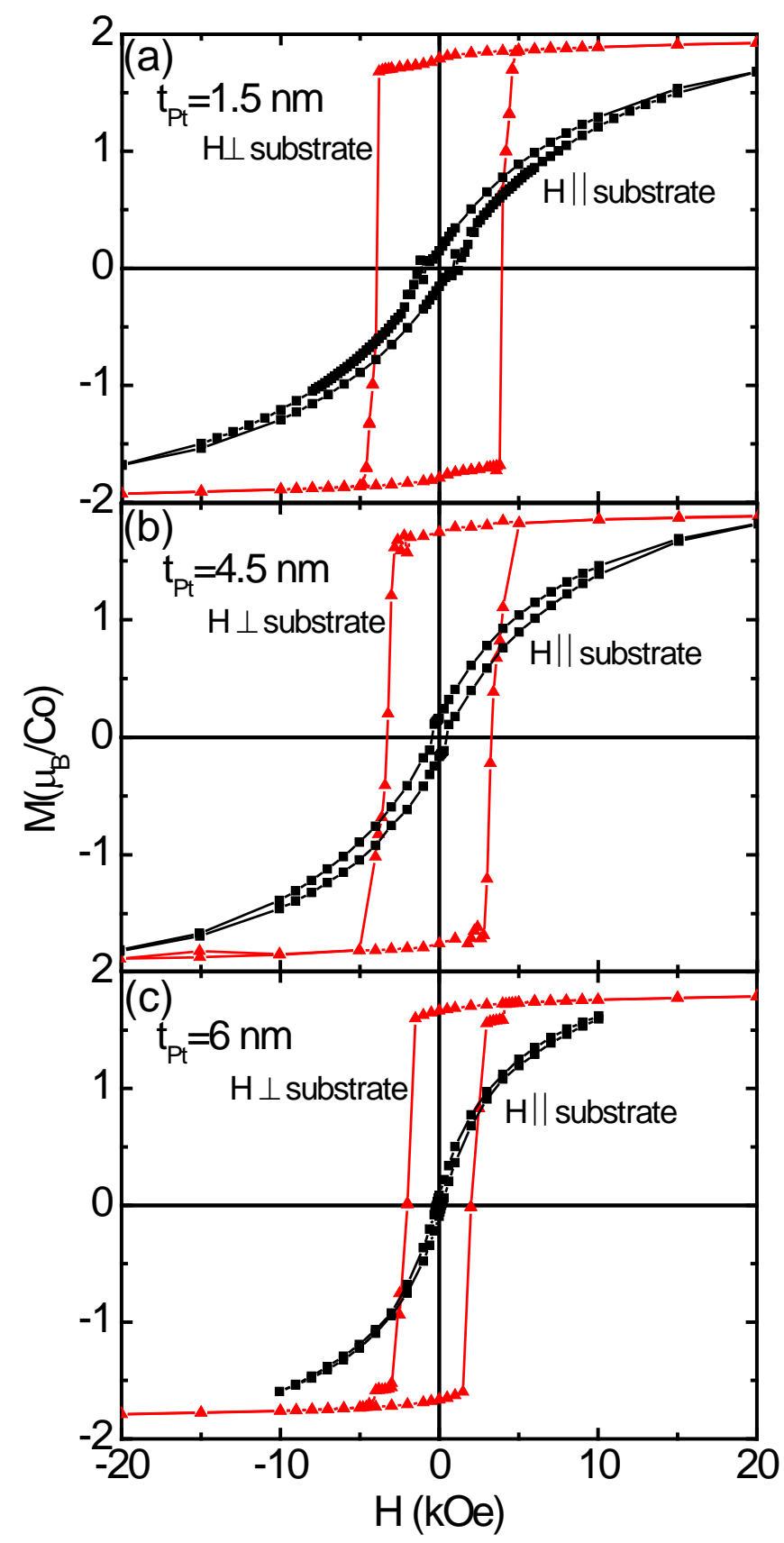

Figure 4.8: Hysteresis loops measured at $T=5 \mathrm{~K}$ and $H_{\mathrm{dc}}$ perpendicular $(\boldsymbol{\Delta})$ and parallel (ם) to the substrate, for the samples with $t_{\mathrm{Co}}=0.7 \mathrm{~nm}, N=25$, and (a) $t_{\mathrm{Pt}}=1.5 \mathrm{~nm}$, (b) $t_{\mathrm{Pt}}=4.5 \mathrm{~nm}$, and (c) $t_{\mathrm{Pt}}=6 \mathrm{~nm}$. 


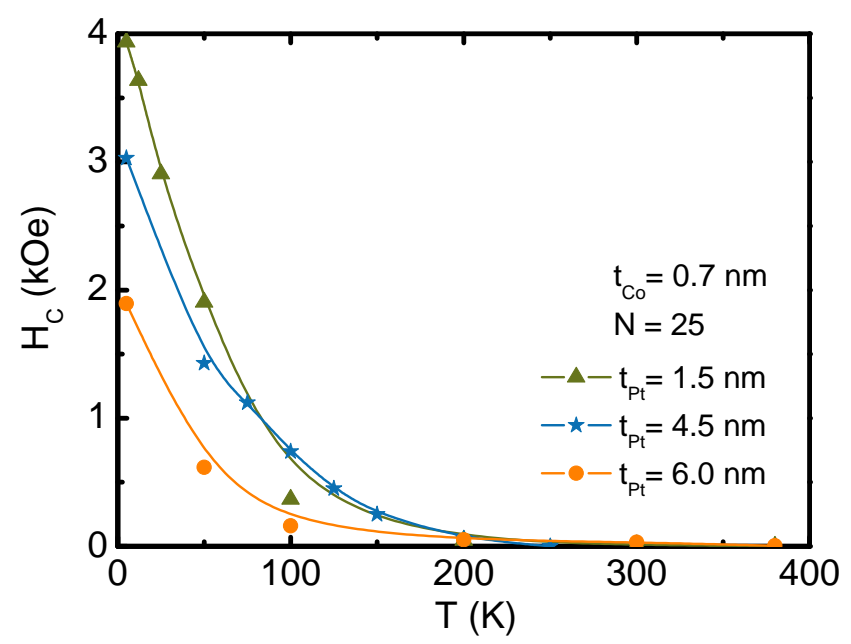

Figure 4.9: Temperature dependence of the coercive field measured with $H_{\mathrm{dc}}$ applied perpendicular to the substrate plane for the samples with $t_{\mathrm{Co}}=0.7 \mathrm{~nm}, N=25$, and (a) $(\Delta) t_{\mathrm{Pt}}=1.5 \mathrm{~nm},(\mathrm{~b})(\star) t_{\mathrm{Pt}}=4.5 \mathrm{~nm}$ and $(\mathrm{b})(\bullet) t_{\mathrm{Pt}}=6 \mathrm{~nm}$.

$t_{\mathrm{Pt}}=6 \mathrm{~nm}$ is higher than that of the sample with $t_{\mathrm{Pt}}=4.5 \mathrm{~nm}$. This, in principle, unusual behavior has also been reported for $\mathrm{Co} / \mathrm{Pt}$ multilayers where an oscillatory dependence of $H_{\mathrm{C}}$ with $t_{\mathrm{Pt}}$ and $N$ is observed, and has been ascribed to RKKY interactions between the Co layers through Pt [147].

There is an interesting feature in the hysteresis curve of the sample with $t_{\mathrm{Pt}}=6 \mathrm{~nm}$ measured in the perpendicular configuration (Fig. 4.8(c)). In the first and third quadrants, the magnetization reversal in this sample apparently proceeds layer by layer. The last layer magnetization reversal involves a variation of $\Delta M_{s} / M_{s}=1 / 12.1$, where $M_{s}$ is the saturation moment. Indeed, if each layer contributes $M_{1}=M_{s} / 25$ to the total magnetization, the reversal of the magnetization of one layer will imply a moment difference of twice this quantity.

In the direction parallel to the substrate plane the coercive field is much lower, being $0.85,0.46$ and $0.15 \mathrm{kOe}$ for samples with $t_{\mathrm{Pt}}=1.5,4.5$ and $6 \mathrm{~nm}$, respectively, at $5 \mathrm{~K}$ (see Fig. 4.8). Such differences between the magnetization curves measured in orthogonal directions (with the bias field applied parallel and perpendicular to the substrate plane) evidence the presence of perpendicular magnetic anisotropy (PMA) in the Co-Pt samples. The easy magnetization direction has been identified to be the one perpendicular to the substrate, as observed in different Co-Pt multilayer systems [148-151], $\mathrm{Co}_{x} \mathrm{Pt}_{1-x}$ alloy films $\lfloor 139-142\rfloor$ and Co-Pt NPs systems [30, 31].

Above $T_{f}$ the $M(H)$ curves measured in both configurations have the characteristic superparamagnetic behavior, which can be fitted to a Langevin curve 
of the nominal Co-Pt particle average diameter $\lfloor 152\rfloor$, following a procedure analogous to the one described in section 2.3.1.

\subsubsection{Anomalous Hall Effect magnetometry}

The anomalous Hall effect (AHE) measurement technique is a method frequently used to study the magnetic properties of thin films exhibiting PMA. Measurements of AHE in ferromagnetic granular systems has demonstrated its sensitivity to the local magnetic moments of individual nano-particles [153], so that hysteresis is developed in the Hall voltage versus applied field curve below the blocking temperature of magnetic particles. Thus, by measuring the variation of the hall voltage $V_{H}$ with the magnetic field applied in the direction perpendicular to the substrate of our Co-Pt NPs samples, hysteresis loops comparable with those of SQUID magnetometry can be obtained.

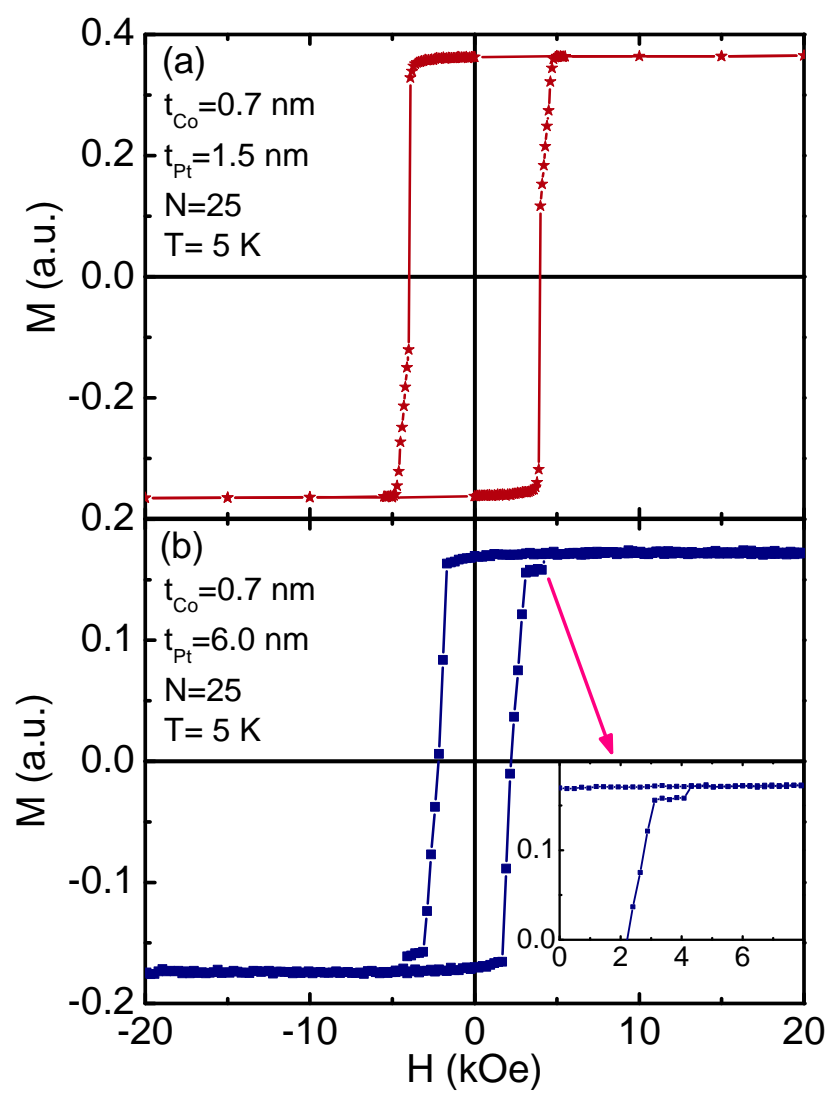

Figure 4.10: AHE hysteresis loops measured at $T=5 \mathrm{~K}$ and $H_{\mathrm{dc}}$ perpendicular to the substrate for the samples with $t_{\mathrm{Co}}=0.7 \mathrm{~nm}, N=25$, and (a) $t_{\mathrm{Pt}}=1.5 \mathrm{~nm}$ and (b) $t_{\mathrm{Pt}}=6 \mathrm{~nm}$. Inset in (b): details of the first quadrant of the hysteresis loop in (b). 


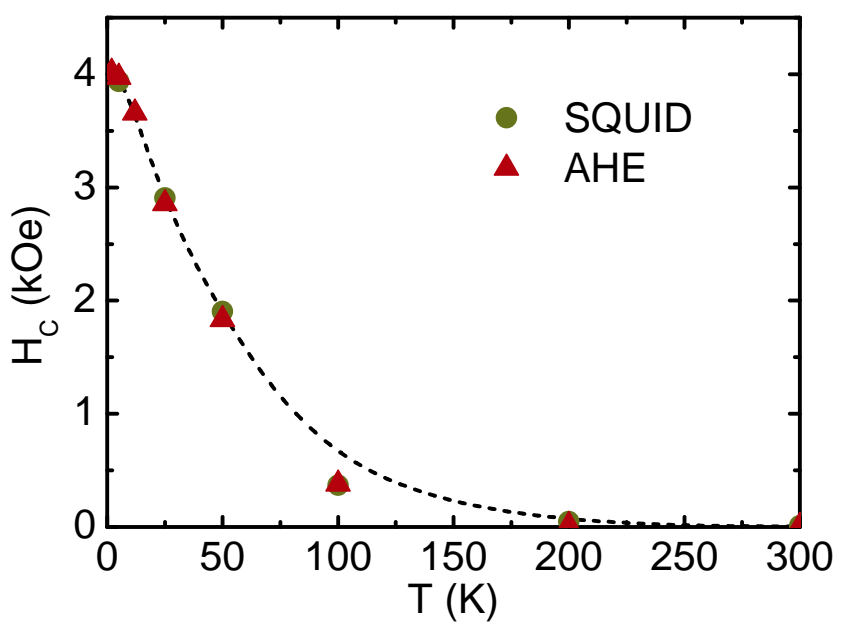

Figure 4.11: Comparison of the $H_{\mathrm{C}}$ vs. $T$ obtained by SQUID $(\bullet)$ and $\operatorname{AHE}(\boldsymbol{\Delta})$ measurements with $H_{\mathrm{dc}}$ applied perpendicular to the substrate plane of the sample with $t_{\mathrm{Co}}=0.7 \mathrm{~nm}$ and $t_{\mathrm{Pt}}=1.5 \mathrm{~nm}$. Dashed line: guide to the eye.

AHE measurements were performed on two of the Co-Pt NPs samples, those with $t_{\mathrm{Co}}=0.7 \mathrm{~nm}$, and $t_{\mathrm{Pt}}=1.5$ and $6.0 \mathrm{~nm}$. These measurements were carried out by Dr. Jolanta Stankiewicz in an electrical transport setup of the Departamento de física de la materia condensada and Instituto de Ciencia de Materiales de Aragón (ICMA), CSIC - Universidad de Zaragoza, and in a Quantum Design Physical Property Measurement System (PPMS) of the Servicio de medidas físicas of the Universidad de Zaragoza. ${ }^{6}$ The measurement configuration is that shown in Fig. 1.3 of section 1.3, with the four contacts placed at the edges of the rectangular samples. The magnetic field was varied within $H= \pm 20 \mathrm{kOe}$, and applied in the direction perpendicular the substrate. $V_{H}$ vs. $H$ loops were collected at several temperatures from $2 \mathrm{~K}$ to $300 \mathrm{~K}$. In Fig. 4.10 we have plotted those $M(H)$ obtained for the two CoPt samples at $T=5 \mathrm{~K}$. These curves show a remarkable squareness and the coercive field coincides well with the one obtained by SQUID magnetometry (see Fig. 4.8). However, the soft component observed in the curves measured in the SQUID magnetometer is not detected by AHE, which instead seems to be very sensitive to the hard component. The $H_{\mathrm{C}}$ values and their temperature dependence obtained by this method perfectly match those observed by SQUID magnetometry in Fig. 4.9, as observed in the direct comparison of $H_{\mathrm{C}}$ vs. $T$ for both techniques depicted in Fig. 4.11.

AHE measurements also allow to examine the magnetization reversal mechanisms in our granular films. The inset of Fig. 4.10(b) reveals details of the

\footnotetext{
${ }^{6}$ http://sai.unizar.es/medidas/index.html
} 
switching of magnetization in the sample with $t_{\mathrm{Pt}}=6.0 \mathrm{~nm}$, which is consistent with the layer by layer reversing described in the previous section for the same sample.

\subsubsection{X-ray Magnetic Circular Dichroism magnetometry}

XANES and XMCD measurements at the Co $K(7709 \mathrm{eV})$, Pt $L_{3}(11564 \mathrm{eV})$ and $\mathrm{Pt} L_{2}(13273 \mathrm{eV})$ edges on the Co-Pt NPs sample with $t_{\mathrm{Co}}=0.7 \mathrm{~nm}$ and $t_{\mathrm{Pt}}=1.5 \mathrm{~nm}$ were performed at the ESRF ID12 beamline. The APPLEII undulator and a double - Si - (111) - crystal monocromator were used to collect the spectra at the respective energies. XANES spectra were recorded by a fluorescence detector in backscattering geometry. Polarization of the circular light was over $90 \%$ in these cases.

XANES and XMCD experiments at he Co $L_{3}(778.1 \mathrm{eV})$ and Co $L_{2}(793.2$ $\mathrm{eV}$ ) edges on the same Co-Pt NPs sample were performed at the ID08 beamline of the ESRF. An APPLE-II undulator and a spherical grating monochromator were used in this case. The degree of polarization at these Co $L_{2,3}$ edges was $\sim 100 \%$ and the total electron yield (TEY) detection method was employed.

The XMCD signal at each edge was obtained by applying a magnetic field of $10 \mathrm{kOe}$ along the X-ray beam direction, at a temperature of $10 \mathrm{~K}$. This field and temperature were chosen according to the magnetic properties of the CoPt NPs, so that the system was reaching its magnetic saturation under these conditions (see section 4.3.1). XMCD was obtained by differences of XANES spectra measured with opposite helicities of the light at a fixed magnetic field value, orienting the field in two inverse directions.

\section{Co $K$ edge results}

XANES and XMCD at the Co $K$ edge probes the $4 p$ empty states of the Co atom in the sample. Analysis of the XANES spectra recorded at this edge on these Co-Pt alloy NPs samples was described in section 4.2.2. Evidences of Co $4 p-3 d$ and Co $3 d-\mathrm{Pt} 5 d$ hybridization in the Co-Pt particles and electronic transfer from $\mathrm{Pt} 5 d$ to Co orbitals were then observed. As described for the XMCD at the Co $K$ edge in the Co-W NPs (section 3.1.1), the detection of a nonzero XMCD signal at this edge reflects the magnetic polarization of the Co $4 p$ band caused by the intra-atomic exchange interaction with the $3 d$ band. The XMCD signal recorded at this edge for the Co-Pt NPs sample is shown in Fig. 4.12, normalized to the absorption jump obtained in the Co $K$ XAS spectrum after background removal. It has the same sign as the Co $K$ XMCD of the Co-W NPs (see section 3.1.1) and as the one reported in Ref. [127], 


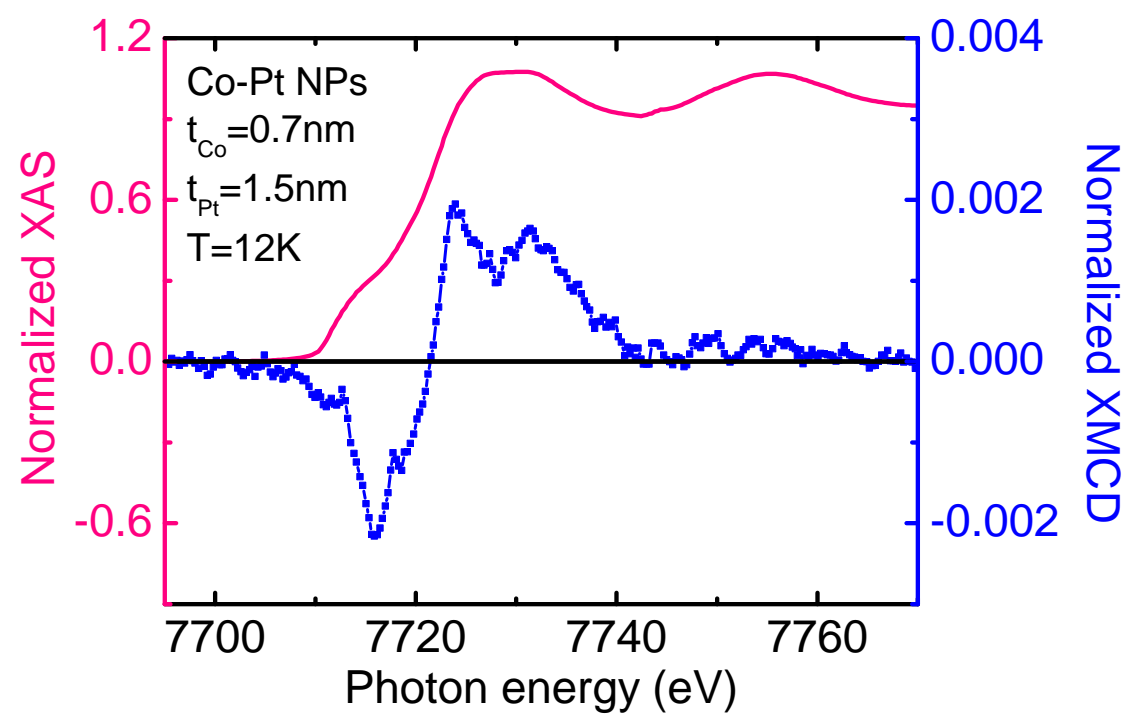

Figure 4.12: (a) (-) XANES spectra, and (b) (ם) XMCD signal at the Co $K$ edge for the Co-Pt NPs with $t_{\mathrm{Co}}=0.7 \mathrm{~nm}$ and $t_{\mathrm{Pt}}=1.5 \mathrm{~nm}$.

which results in an antiferromagnetic coupling between $4 p$ and $3 d$ levels in Co.

The shape of XMCD signal in Fig. 4.12 is indeed very similar to the one reported for ion irradiated Co-Pt films in Ref. [154]. In these Co-Pt films, changes in the electronic structure of Co atoms related to the formation of $\mathrm{Co}_{x} \mathrm{Pt}_{1-x}$ alloys induced by ion irradiation were observed. Striking similarities between the XMCD spectra of the Co-Pt films and a reference $\mathrm{Co}_{0.5} \mathrm{Pt}_{0.5} L 1_{0}$ thin-film reference sample were shown, in terms of characteristic peaks at selected energies. This allowed to interpreted the XMCD spectrum for the irradiated Co-Pt film as the superposition of pure Co and $L 1_{0} \mathrm{Co}_{0.5} \mathrm{Pt}_{0.5}$ alloy contributions. Similarly, in our Co-Pt NPs we may state that the Co $K$ edge XMCD signal that we observe is a combination of these two phases, since we have demonstrated the existence of both by EXAFS analysis (see section 4.2.2).

By comparing the amplitude of the Co $K$ edge XMCD curve for the CoPt NPs with that of Ref. [127], we have estimated the $4 p$ Co moment to be $-0.057(4) \mu_{B}$. This value has been reduced in about $35 \%$ with respect to that found for the $\mathrm{Al}_{2} \mathrm{O}_{3}$ capped Co particles $\left(m^{4 p}=-0.087[11\rfloor\right)$. Such a reduction may be a consequence of the Co-Pt alloy present in the samples. 


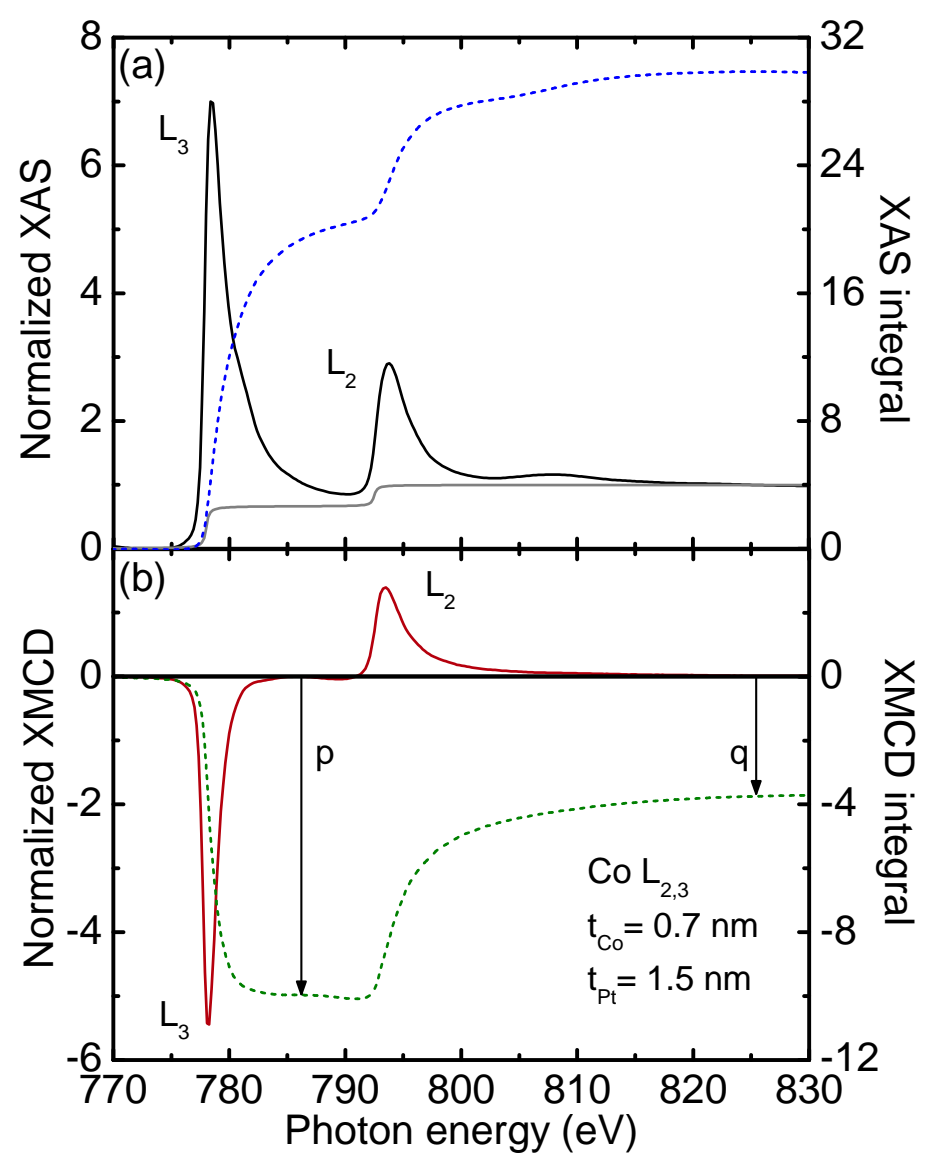

Figure 4.13: $\mathrm{Co}-L_{2,3}$ edges spectra of the $t_{\mathrm{Co}} 0.7 \mathrm{~nm}, t_{\mathrm{Pt}}=1.5 \mathrm{~nm}$ sample. (a) (-) Normalized XAS spectra mean curve. (-) The double-step function used is also indicated. (- -) Integrated white line. (b) (-) Normalized XMCD spectra, (- -) integrated area. The higher limit for the $\mathrm{p}$ and $\mathrm{q}$ integrals, used for the sum rules analysis, is given in (b).

\section{Co $L_{2,3}$ edges results}

Figure 4.13 shows the XMCD spectrum measured at the Co $L_{2,3}$ for normal incidence of the X-rays. This spectrum has been normalized to the XAS shown in Fig. 4.13(a) in high energy limit, after background subtraction.

As described in previous chapters, in order to apply the magneto-optical sum rules $[64,65\rfloor$ (see section 1.4.2) to the XMCD signal in Fig. 4.13(b), the number of unoccupied states in the $3 d$ band for Co $n_{h}$ is required in the calculations. Values of $3 d n_{h}$ reported in the literature are, for example, $n_{h}=2.49$ holes for metallic Co $\lfloor 108\rfloor$ and $n_{h}=2.628$ for $\mathrm{Co}_{0.5} \mathrm{Pt}_{0.5} L 1_{0}$ alloy films $\lfloor 141$. Let us recall that the Co atoms in our Co-Pt NPs are found to be 
in these two different environments (metallic Co and $\mathrm{Co}_{0.5} \mathrm{Pt}_{0.5} L 1_{0}$ alloy), but we are not able to quantify each of them. Therefore, the right number of $3 d$ holes in Co atoms of our Co-Pt NPs is unknown. Thus, we have expressed the orbital and spin moments, per $3 d$ hole in the Co atoms, as $m_{\mathrm{S}} / n_{h}=0.80(1)$ $\mu_{B}, m_{\mathrm{L}} / n_{h}=0.09(1) \mu_{B}$, and $m_{\mathrm{L}} / m_{\mathrm{S}}=0.11(1)$. The spin moment $m_{\mathrm{S}} / n_{h}$ has been obtained considering that the dipolar term $m_{\mathrm{D}}$ is small with respect to the total $m_{\mathrm{S}}$ (see section 1.4.2).

XMCD measurements at the Co $L_{2,3}$ edges on $\mathrm{Co}_{x} \mathrm{Pt}_{1-x}$ particles prepared by vapor deposition have been recently reported $[155,156]$. They find systematically larger ratios of $m_{\mathrm{L}} / m_{\mathrm{S}}$ than those in our Co-Pt NPs, for comparable particle sizes. In that case, the orbital to spin ratio $m_{\mathrm{L}} / m_{\mathrm{S}}$ of Co atoms in our Co-Pt NPs is close to the value found for particles of $2.0 \mathrm{~nm}$ with composition close to $\mathrm{Co}_{3} \mathrm{Pt}[156$. With reference to other XMCD studies in Co-Pt systems, our $m_{\mathrm{L}} / m_{\mathrm{S}}$ in Co is always larger, as compared to $\mathrm{CoPt}_{3}$ films deposited on a substrate at $800 \mathrm{~K}\left(m_{\mathrm{L}} / m_{\mathrm{S}}=0.09\right)\lfloor 140\rfloor, \mathrm{Co}_{0.5} \mathrm{Pt}_{0.5} L 1_{0}$ alloy films $\left(m_{\mathrm{L}} / m_{\mathrm{S}}=0.063\right)\lfloor 141\rfloor$, and for annealed $\mathrm{Co}_{0.5} \mathrm{Pt}_{0.5} \mathrm{NPs}$ with diameters of about $2.6 \mathrm{~nm}$ deposited on amorphous carbon matrices $\left(m_{\mathrm{L}} / m_{\mathrm{S}}=0.094\right)$ 22 .

XMCD measurements at the Co $L_{2,3}$ edges as a function of incident angle $\left(0^{\circ}\right.$ and $\left.60^{\circ}\right)$ were also performed, looking for anisotropy of the orbital moment. However, the results did not show clear angle dependence, which was unexpected.

\section{Pt $L_{2,3}$ edges results}

The XMCD spectra measured at the $\mathrm{Pt} L_{2,3}$ edges are shown in Fig. 4.14. The non-zero values of the XMCD signal reflects the polarization of the Pt by the magnetic Co. From an XMCD experiment at the $\mathrm{Pt} L_{2,3}$ edges one obtains, through the sum rules $\lfloor 64,65\rfloor$, the spin $m_{\mathrm{S}}$ and orbital $m_{\mathrm{L}}$ moments averaged over the whole sample, provided one knows by calculation or estimation the number of holes, $n_{h}$, existing involved in the $2 p \rightarrow 5 d$ excitations. For Pt we have derived $n_{h}$ from the white line difference with a $\mathrm{Pt}$ foil at the $L_{2,3}$ edges XANES, similarly to the procedure followed for XANES at the W $L_{2,3}$ edges in section 3.1.2. We have obtained $n_{h}=1.80(2)$ holes in the $5 d$ band, $m_{\mathrm{S}}=0.14(1) \mu_{B}, m_{\mathrm{L}}=0.027(2) \mu_{B}$, and $m_{\mathrm{L}} / m_{\mathrm{S}}=0.19(1)$ for Pt atoms in the Co-Pt NPs.

The induced moment on $\mathrm{Pt} m_{\mathrm{tot}}=0.16(1) \mu_{B}$ is one order of magnitude larger than the moments induced on the $\mathrm{Cu}, \mathrm{Ag}$ or $\mathrm{Au}$ capping cases [11]. Obviously, the reason for this difference is the proximity of $\mathrm{Pt}$, with the $5 d^{9}$ band, to fulfill the Stoner criterion for ferromagnetism [157, instead of the 


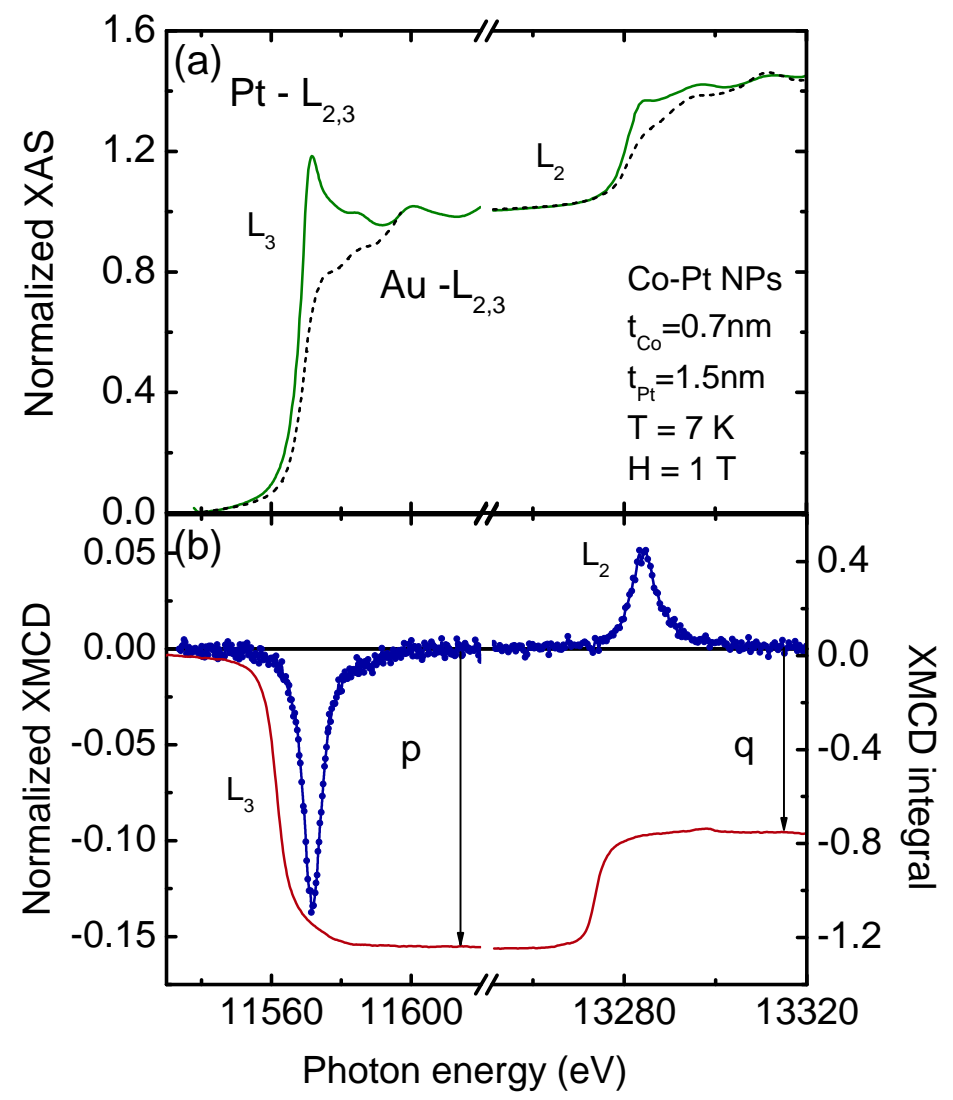

Figure 4.14: (-) Normalized XANES (a) and (•) XMCD (b) at the Pt $L_{2,3}$ edges in the Co-Pd NPs. (-) XMCD integrated area. (- -) The XANES spectrum of Au $L_{2,3}$ edges is shown in (a) for comparison. The higher limit for the $\mathrm{p}$ and $\mathrm{q}$ integrals, used for the sum rules analysis, is given in (b).

nearly filled noble metal $n d^{10}$ bands $(n=3,4$ and 5 for $\mathrm{Cu}, \mathrm{Ag}$ or $\mathrm{Au}$, respectively).

However, it is important to point out that not all $\mathrm{Pt}$ atoms in the Co-Pt NPs of the measured sample $\left(t_{\mathrm{Co}}=0.7 \mathrm{~nm}\right.$ and $\left.t_{\mathrm{Pt}}=1.5 \mathrm{~nm}\right)$ are alloyed or in contact with Co atoms, as described in the HRTEM and EXAFS measurements (see section 4.2) performed in this sample. As a result, it is possible that not all $\mathrm{Pt}$ atoms in the sample are polarized by Co. Only those polarized $\mathrm{Pt}$ atoms contribute to the $\mathrm{Pt} L_{2,3}$ XMCD signal, but all $\mathrm{Pt}$ atoms in the sample are reflected in the Pt $L_{2,3}$ XAS. Since the XMCD results are scaled to the Pt $L_{2,3}$ XAS spectra, the $m_{\mathrm{S}}$ and $m_{\mathrm{L}}$ results from Pt XMCD may be lower than the actual ones. The $m_{\mathrm{L}} / m_{\mathrm{S}}$ ratio depends only on the XMCD integral values (see section 1.4.2), so it is not affected by the Pt $L_{2,3}$ XAS scale and we may rely on that value. 
Comparing to XMCD measurements at the $\mathrm{Pt} L_{2,3}$ edges reported in the literature, the $m_{\mathrm{L}} / m_{\mathrm{S}}$ ratio in the $5 d$ band of $\mathrm{Pt}$ in our Co-Pt NPs is close to

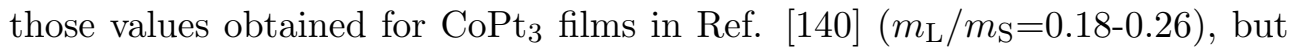
lower than those of $\mathrm{Co}_{0.5} \mathrm{Pt}_{0.5} L 1_{0}$ alloy films $\left(m_{\mathrm{L}} / m_{\mathrm{S}}=0.27\right)\lfloor 141\rfloor$.

\section{Element-specific magnetic hysteresis measured by XMCD}

One important advantage of the selective magnetometry using the XMCD technique is the possibility of performing element-specific magnetic hysteresis measurements. It consists in recording the dichroic signal for a fixed energy while the applied magnetic field is varied, similarly to the magnetization hysteresis measurements with conventional magnetometers. This way it is possible to evaluate the contribution of each atomic species to the magnetic properties of the material. We have performed $\operatorname{XMCD}(H)$ at the Co $K$ and $\mathrm{Pt} L_{3}$ edges on the same sample of Co-Pt NPs with $t_{\mathrm{Co}}=0.7 \mathrm{~nm}$ and $t_{\mathrm{Pt}}=1.5 \mathrm{~nm}$. Measurements were done at the ESRF ID12 beamline, with the same technical details than in the conventional XMCD experiments described above.

Figure 4.15 shows the XMCD spectra recorded at $12 \mathrm{~K}$ in normal incidence at the $\mathrm{Co} K$ and $\mathrm{Pt} L_{2,3}$ edges, from which $\operatorname{XMCD}(H)$ measurements have been performed. The energies at which $\operatorname{XMCD}(H)$ were collected were 7723.9 $\mathrm{eV}$ and $11571.5 \mathrm{eV}$ for Co $K$ and $\mathrm{Pt} L_{3}$ edges, respectively (see arrows in Fig. 4.16). The insets in Fig. 4.16 illustrate the curves obtained for each edge. Notice that both hysteresis loops are squared, with a coercive field around $3.6 \mathrm{kOe}$ in both cases, which are consistent with those curves measured by SQUID (section 4.3.1) and AHE (section 4.3.2) techniques. The agreement in the $H_{\mathrm{C}}$ values obtained by $\operatorname{XMCD}(H)$ is an indicative that Co and $\mathrm{Pt}$ atoms in the Co-Pt NPs are strongly magnetically coupled, as resulting from
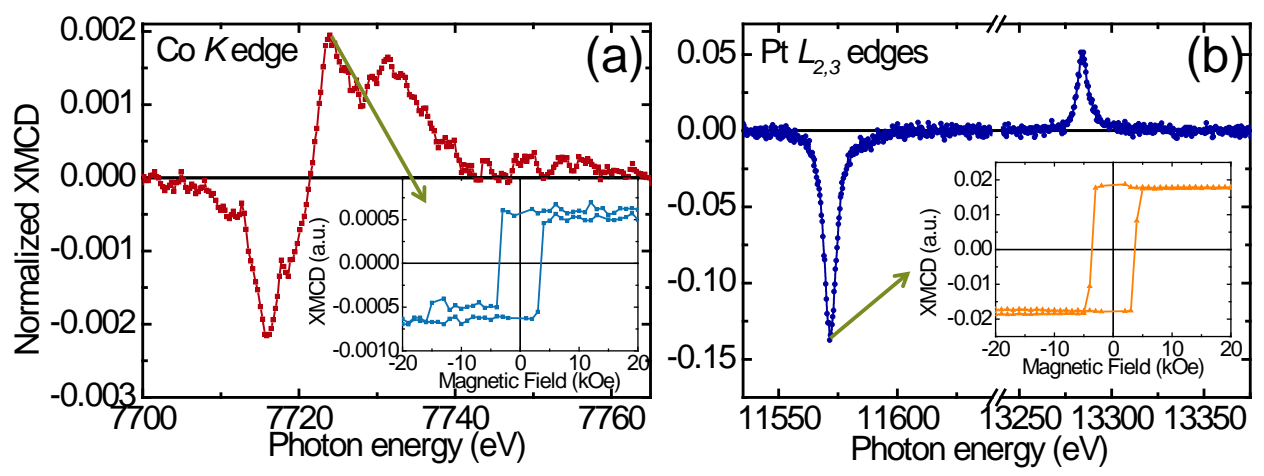

Figure 4.15: XMCD signal and magnetic hysteresis loops measured at the selected energy in the Co-Pt NPs with $t_{\mathrm{Co}}=0.7 \mathrm{~nm}$ and $t_{\mathrm{Pt}}=1.5 \mathrm{~nm}$ : (a) Co $K$ edge and (b) Pt $L_{2,3}$ edges. 


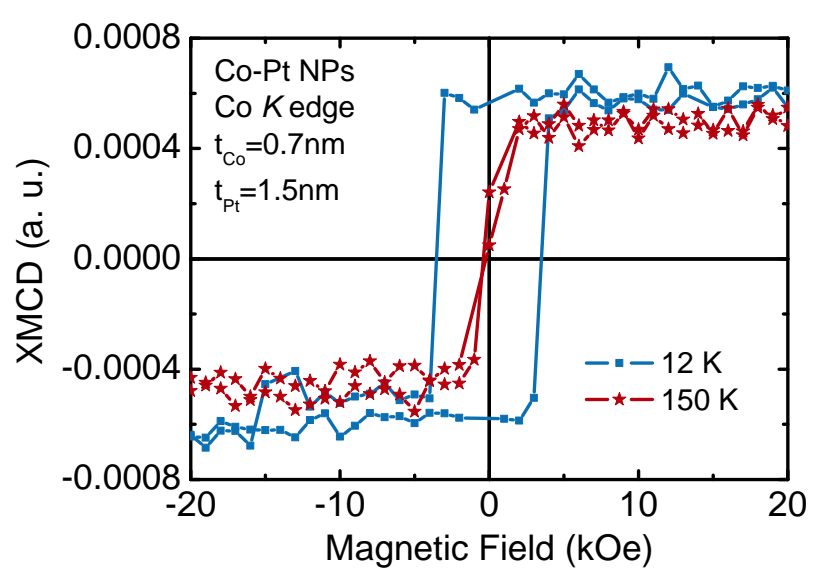

Figure 4.16: Magnetic hysteresis loops measured at $T=12 \mathrm{~K}(\square)$ and $150 \mathrm{~K}(\star)$ at the selected energy of the XMCD Co $K$ edge in the Co-Pt NPs with $t_{\text {Co }}=0.7 \mathrm{~nm}$ and $t_{\mathrm{Pt}}=1.5 \mathrm{~nm}$.

the Co-Pt alloy in the sample. Both Co and Pt atoms are responsible of the magnetic hard component measured in the sample with the magnetic field in the perpendicular configuration. No clear observation of the soft component has been detected in these measurements.

The decay of the $H_{\mathrm{C}}$ values with increasing temperature has been also examined from the $\mathrm{XMCD}(H)$, by measuring the hysteresis loop at the Co $K$ edge at $T=150 \mathrm{~K}$. In Fig. 4.16 we have plotted the $\operatorname{XMCD}(H)$ curves at $12 \mathrm{~K}$ and $150 \mathrm{~K}$ to illustrate the reduction in $H_{\mathrm{C}}$, from $3.6 \mathrm{kOe}$ down to $0.39 \mathrm{kOe}$. This behavior is consistent with that observed by SQUID and AHE measurements (see Fig. 4.11).

\subsection{Discussion}

The formation of a $\mathrm{Co}_{x} \mathrm{Pt}_{1-x}$ alloy in our Co-Pt NPs has been proven by XANES and EXAFS measurements at the Co $K$ and $\mathrm{Pt} L_{3}$ edges. It results from the interdiffusion of Co atoms from the previously formed Co clusters towards the Pt matrix, as observed in other Co-Pt systems produced by sputtering $\lfloor 142\rfloor$ and low energy cluster beam deposition $\lfloor 158\rfloor$ at room temperature. This alloy gives rise to a quite different magnetic behavior than that observed for the noble metal-capped Co NPs $\lfloor 11\rfloor$ and Co-W NPs (see chapter 2). Co-Pt NPs form in each layer a bidimensional array of particles with a ferromagnetic behavior below a characteristic temperature $T_{f}$, identified from the peak in the $M(T)$ curve measured in the parallel configuration and the shoulder in the curve measured in the perpendicular one. This result contrasts with the 


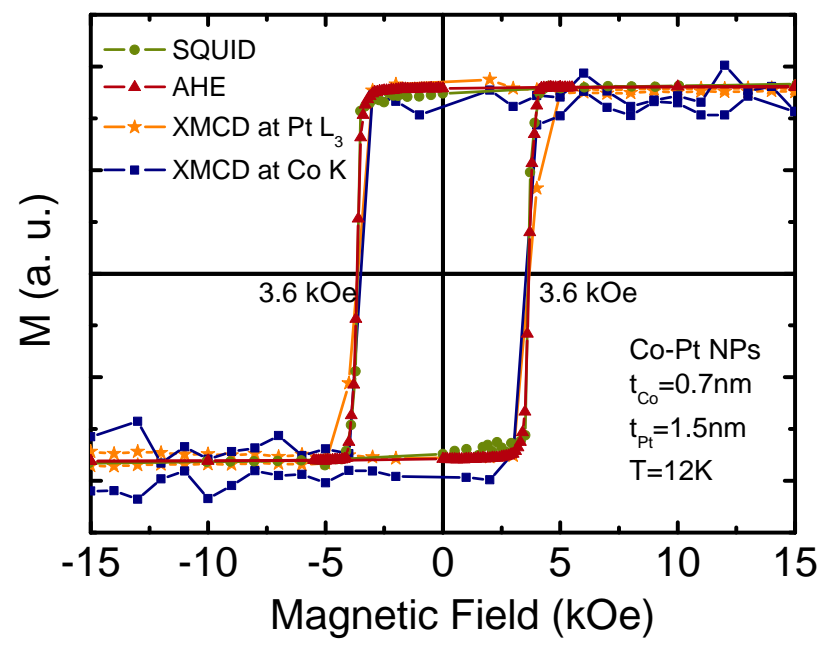

Figure 4.17: Comparison of the magnetic hysteresis loops measured at $T=12 \mathrm{~K}$ with different techniques in the Co-Pt NPs with $t_{\mathrm{Co}}=0.7 \mathrm{~nm}$ and $t_{\mathrm{Pt}}=1.5 \mathrm{~nm}:(\bullet)$ SQUID magnetometry, $(\boldsymbol{\Delta}) \mathrm{AHE},(\star)$ XMCD at the Pt $L_{3}$ edge, and (ם) XMCD at the Co $K$ edge.

blocked superparamagnetic behavior observed in Co-M NPs with $\mathrm{M}=\mathrm{Cu}, \mathrm{Ag}$, $\mathrm{Au}$ and $\mathrm{W} . T_{\mathrm{f}}$ depends on the average particle size, and above $T_{\mathrm{f}}$ the Co-Pt NPs are superparamagnetic.

The different magnetometry techniques employed to study the Co-Pt NPs reveal the presence of PMA in all the samples in the series. There is evidence of two different contributions, a soft and a hard magnetic component. For the latter, the coercive field is found to decrease with increasing temperature, being zero at a temperature of $T_{1} \approx 140 \mathrm{~K}$ in all samples. This suggests that the magnetic hard component is the same in all samples in the series.

This magnetic hard component is observed in all magnetometry techniques employed in the present study. This can be observed in Fig. 4.17, where the comparison of the hysteresis loops obtained for the Co-Pt NPs sample with $t_{\mathrm{Co}}=0.7 \mathrm{~nm}$ and $t_{\mathrm{Pt}}=1.5 \mathrm{~nm}$ in the perpendicular configuration at $T=12 \mathrm{~K}$ is depicted. The y-axis has been scaled for all curves to coincide. The magnetic hard component is evidenced from the rather squared curves and the high $H_{\mathrm{C}}$ values obtained from all measurements. These curves demonstrate that the Co-Pt NPs are strongly magnetically coupled, as resulting from the Co-Pt alloy in the sample.

The composition of the $\mathrm{Co}_{x} \mathrm{Pt}_{1-x}$ alloy present in all Co-Pt NPs samples seems to be close to $\mathrm{Co}_{0.5} \mathrm{Pt}_{0.5}$, according to the EXAFS results. Co-Pt alloys are known to have a variety of magnetic properties depending on their composition and crystallinity $\lfloor 159\rfloor$. For example, the crystallographically ordered 
alloy $\mathrm{CoPt}_{3}$ has a Curie temperature of $T_{\mathrm{C}}=290 \mathrm{~K}$. A departure from the exact composition or lack of perfect crystallinity would reduce it to $T_{\mathrm{C}} \approx 200$ $\mathrm{K}$, which is the temperature of disappearance of coercivity in our samples. CoPt alloys with composition close to $\mathrm{Co}_{0.5} \mathrm{Pt}_{0.5}$ may have a disordered $f c c$ structure or a chemically ordered tetragonal structure $L 1_{0}$. Both phases are ferromagnetic and the $\mathrm{C}$ axis of the $L 1_{0}$ is an easy direction of magnetization. CoPt films grown with this $L 1_{0}$ phase show PMA [141]. Therefore, one may identify the PMA appearing on our Co-Pt NPs as due to the formation of $\mathrm{Co}_{0.5} \mathrm{Pt}_{0.5}$ alloy with short range ordering in the $L 1_{0}$ structure.

The XMCD data also point towards the same explanation by comparison with measurements on different Co-Pt systems in the literature. Thus, a $\mathrm{Co}_{0.5} \mathrm{Pt}_{0.5}$ alloy (hard magnetic component) is probably surrounding the Co rich core (soft magnetic component), in view of the particulate morphology of the Co-Pt layers observed by TEM and the structure described by EXAFS measurements.

Recent calculations of core-shell morphologies in CoPt nanoparticles have demonstrated that the Co core $-\mathrm{Co}_{0.5} \mathrm{Pt}_{0.5}$ alloy shell, with some segregation of $\mathrm{Pt}$ atoms to the particle surface, is one of the most energetically stable configurations of NPs with diameter close to $3 \mathrm{~nm}$ (clusters of $\approx 1000$ atoms) $\lfloor 160,161\rfloor$. Those calculations have also allowed to estimate the spin magnetic moments of $\mathrm{Co}$ and $\mathrm{Pt}$ in the clusters, resulting in values of $m_{\mathrm{S}}^{\mathrm{Co}}=1.85-1.96$ $\mu_{B} /$ Co atom and $m_{\mathrm{S}}^{\mathrm{Pt}}=0.26-0.42 \mu_{B} / \mathrm{Pt}$ atom. Since we are not able to obtain the absolute spin moment in Co in our Co-Pt NPs, the comparison

with those $m_{\mathrm{S}}^{\mathrm{Co}}$ values is not possible. In the case of $m_{\mathrm{S}}^{\mathrm{Pt}}$, the deviation of the $m_{\mathrm{S}}^{\mathrm{Pt}}=0.14(1) \mu_{B}$ value for our Co-Pt NPs from that calculated in Refs. $\lfloor 160,161\rfloor$ may be related to the difference between the actual amount of $\mathrm{Pt}$ in the samples that is polarized by Co and contributes to the XMCD signal, as described in section 4.3.3.

\subsection{Conclusions}

The study that we have described in this chapter allowed to characterize the system of self-organized Co-Pt NPs. The overall picture describing the collective behavior of these multilayers is that of a Co-core with a Co-Pt alloy shell particles, embedded in the non-reacted $\mathrm{Pt}$, strongly coupled via the polarized $\mathrm{Pt}$ or via dipolar or RKKY interaction. The polarization of the $\mathrm{Pt}$ atoms is observed by XMCD measurements and has been demonstrated to have an important role in the magnetic behavior of the Co-Pt NPs.

The short range order within the grains would create the anisotropy in 
the $\mathrm{Co}_{0.5} \mathrm{Pt}_{0.5}$ alloy which would give rise to the PMA. Moreover, the sample preparation may play an important role, as demonstrated from comparison with results of Co-Pt NPs systems prepared by different chemical and physical methods $[155,156\rfloor$. 


\section{Chapter 5}

\section{d-band magnetism of Ag, Au, $\mathrm{Pd}$ and $\mathrm{Pt}$ nanostructured materials}

In this chapter we review the interesting magnetic properties of metals (M) that are not magnetic in bulk form, but when shaped in reduced dimensions. In particular, we describe the magnetic behavior of $\mathrm{Ag}, \mathrm{Au}, \mathrm{Pd}$ and $\mathrm{Pt}$ nanoparticles. They have in common an incompletely filled $4 d-(\mathrm{Ag}, \mathrm{Pd})$ or $5 d$-band $(\mathrm{Au}, \mathrm{Pt})$, that gives rise partially to this type of magnetism. By means of X-ray magnetic circular dichroism (XMCD) measurements at the Metal- $L_{2,3}$ edges, the orbital and spin contributions to the magnetic moment stemming from the $d$-band are explored.

\subsection{Magnetism in bulk metals}

The understanding of the magnetism of metals has attracted the attention of physicists for decades, and, as a result, very successful theories and models have been proposed to explain it. The magnetic properties of a metal in the solid state in bulk scale may be rationalized in terms of the spin-split density of states DOS. The Stoner criterion of stability with respect to ferromagnetism in metals states that long range ordering is possible only when the Stoner parameter (repulsion between electrons of opposite spin) $I$, times the density of states at the Fermi energy $D\left(\epsilon_{F}\right)$ is larger than 1 [157].

As free atoms, the noble metals $\mathrm{Ag}$ and $\mathrm{Au}$, and $\mathrm{Pd}$ have a full electronic $d$ band, Ag:[Kr] $4 d^{10} 5 s^{1}$, Au:[Xe $4 f^{14} 5 d^{10} 6 s^{1}$ and $\mathrm{Pd}:[\mathrm{Kr}] 4 d^{10}$, respectively, while Pt has a hole in the $5 d$ orbit; Pt:[Xe] $4 f^{14} 5 d^{9} 6 s^{1}$. In the solid state, though, 
$\mathrm{Ag}$ and $\mathrm{Au}$ metals have a very shallow $D\left(\epsilon_{F}\right)$ and, correspondingly, are nonmagnetic, on one hand; Pd and Pt have a maximum in $D\left(\epsilon_{F}\right)$ but do not fulfill the Stoner criterion and remain paramagnetic, on the other hand. This is due to the hybridization between the $d$-band and the $s p$-bands, which generates a small, albeit non-negligible number of holes, $n_{h}^{d}$, in the $d$-band. This parameter varies between $n_{h}^{d}=0.349$ holes for $\mathrm{Ag}$ and $n_{h}^{d}=1.635$ holes for $\mathrm{Pt}$, and increases in the trend $\mathrm{Ag}<\mathrm{Au}<\mathrm{Pd}<\mathrm{Pt}$ (see table 5.1). As a result, the four metals have a partially filled $d$-band.

In non-magnetic metals the magnetic susceptibility response to an applied field can be explained as due to several contributions:

$$
\chi_{\text {total }}=\chi_{\text {dia }}+\chi_{\text {Landau }}+\chi_{\text {Pauli }}^{s}+\chi_{o r b}^{S O}+\chi_{o r b}^{K O}
$$

$\chi_{d i a}$ is the diamagnetic susceptibility of the electrons in the core states, $\chi_{\text {Landau }}$ is the diamagnetic contribution due to the conduction band that splits into discrete Landau transversal orbits upon application of an external field, $\chi_{\text {Pauli }}^{s}$ is the Pauli paramagnetism due to the spin density near the Fermi energy, $\chi_{\text {orb }}^{S O}$ is the paramagnetic orbital component due to spin-orbit (SO) coupling, $\chi_{o r b}^{K O}$ is the Kubo-Obata paramagnetic contribution due to second order perturbation contribution of the itinerant electrons $\lfloor 162\rfloor$.

Bulk Ag and Au have an overall diamagnetic response to an applied field; i.e., the diamagnetic terms overpower the paramagnetic ones. In contrast, in $\mathrm{Pd}$ and $\mathrm{Pt}$, which are paramagnetic, the opposite situation occurs.

The approximation of considering the electron band structure as a $d$-band combined with a conduction band is quite reasonable in these metals, where electrons in the $d$-band play a role of paramount importance. Therefore, the XMCD spectroscopy at the $L_{2,3}$ edges, which excites electrons from a $p$ core level to the empty states of $d$-band, is the ideal experimental tool to probe the $d$-band magnetism, since the spin and orbital contributions are discriminated. One must be aware, though, that dipole excitation from $2 p$ to $(n+1) s$ states cannot be ruled out and may give a non-negligible contribution. Besides, the diamagnetic contribution is not observed.

A recent work on Au foil illustrates this approach beautifully [163]. The $\mathrm{XMCD}$ at the $\mathrm{Au} L_{2,3}$ edges were measured as a function of field up to $H=100$ $\mathrm{kOe}$, at temperatures ranging between 2.2 and $300 \mathrm{~K}$. The XMCD spectra were clearly non-zero, indicating that an induced moment appears at the $5 d$ band and that it aligns parallel to the applied field. Moreover, the magnetic orbital and spin moments were found to depend linearly with the applied field. The orbital to spin moment ratio amounts to $m_{\mathrm{L}} / m_{\mathrm{S}}=0.28$, thus indicating an important orbital contribution to the susceptibility. The spin Pauli para- 
Table 5.1: Collected data for bulk Ag, $\mathrm{Au}, \mathrm{Pd}$ and $\mathrm{Pt}$ metals. $n_{h}^{d}$, number of holes at the d-band; $m_{\mathrm{L}} / m_{\mathrm{S}}$ ratio obtained from the XMCD $L_{2,3}$ edges analysis; $\chi_{\text {total }}$ total magnetic susceptibility measured with conventional magnetometry; $\chi_{d}$, $d$-band susceptibility, from XMCD magnetometry; $\chi_{\text {Pauli }}^{s}$, Pauli paramagnetic spin component; $\chi_{\text {orb }}$ orbital component.

\begin{tabular}{ccccc}
\hline \hline Metal & $\mathrm{Ag}(4 d)$ & $\mathrm{Au}(5 d)^{\mathrm{d}}$ & $\mathrm{Pd}(4 d)^{\mathrm{e}}$ & $\mathrm{Pt}(5 d)^{\mathrm{e}}$ \\
\hline$n_{h}^{d}$ & $0.349^{\mathrm{a}}$ & $0.617^{\mathrm{b}}$ & $1.27^{\mathrm{c}}$ & $1.635^{\mathrm{b}}$ \\
$m_{\mathrm{L}} / m_{\mathrm{S}}$ & & $0.28^{\mathrm{d}}$ & $0.30^{\mathrm{e}}$ & $0.38^{\mathrm{e}}$ \\
$\chi_{\text {total }}(\mathrm{emu} / \mathrm{mol})^{\mathrm{f}}$ & $-1.95 \times 10^{-5}$ & $-2.08 \times 10^{-5}$ & $5.56 \times 10^{-4}$ & $1.89 \times 10^{-4}$ \\
$\chi_{d}(\mathrm{emu} / \mathrm{mol})$ & & $7.0 \times 10^{-6}$ & $1.3 \times 10^{-3}$ & $6.1 \times 10^{-4}$ \\
$\chi_{\text {Pauli }}^{s}(\mathrm{emu} / \mathrm{mol})$ & & $5.4 \times 10^{-6}$ & $9.6 \times 10^{-4}$ & $4.4 \times 10^{-4}$ \\
$\chi_{\text {orb }}(\mathrm{emu} / \mathrm{mol})$ & & $1.5 \times 10^{-6}$ & $3.2 \times 10^{-4}$ & $1.7 \times 10^{-4}$ \\
\hline \hline
\end{tabular}

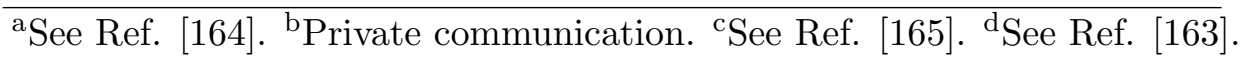

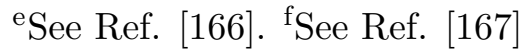

magnetism and orbital components to the susceptibility could be deduced, and the resulting susceptibility due to the $5 d$ band is compared to the total susceptibility in table 5.1 .

The bulk Pd XMCD spectra, at the Pd $L_{2,3}$ edges, up to $H=70 \mathrm{kOe}$ and $4<T<300 \mathrm{~K}$, were also measured [166」. The field dependence is linear, and the susceptibility decreases with increasing temperature. The two components, spin Pauli paramagnetic and orbital susceptibility were deduced and are compared to the rest in table 5.1. The orbital component could be segregated into the $\mathrm{SO}$ and $\mathrm{KO}$ terms by comparing the results with a $\mathrm{Fe} / \mathrm{Pd}$ multilayer. The XMCD at the $L_{2,3}$ edges of bulk Pt were measured likewise [34」 (see table 5.1).

From table 5.1 several conclusions may be drawn. In Au, diamagnetism is dominant, but the paramagnetic $d$-band susceptibility is nevertheless observable. In $\mathrm{Pd}$ and $\mathrm{Pt}$ the $d$-band paramagnetism is partially compensated by the diamagnetic terms. Except for Ag where no data exists, the $d$-band magnetism of the bulk metals is paramagnetic and is attributed to the non-zero number of holes induced by hybridization of this band with the $s-p$ ones. Moreover, the $m_{\mathrm{L}} / m_{\mathrm{S}}$ ratio increases with the number of holes in the $d$-band.

\subsection{Induced magnetism in nanoparticle matrix}

When any of the four metals is in contact with a strongly magnetic material, the interfacial atoms of $\mathrm{M}=\mathrm{Ag}, \mathrm{Au}, \mathrm{Pd}$ and $\mathrm{Pt}$ may also become magnetic in absence of an external applied field. This phenomenon has been extensively 
studied in $\mathrm{T} / \mathrm{M}$ thin films, where $\mathrm{T}$ is a transition metal as $\mathrm{Ni}$, Fe or Co. The case between the interfacial $\mathrm{T}=\mathrm{Ni}$ and $\mathrm{M}=\mathrm{Pt}$ is described in [137], where three possible mechanisms for the induction of magnetic moment atoms are discussed: (a) charge transfer by hybridization between $\mathrm{T}$ and $\mathrm{M} d$-bands, (b) symmetry breaking at the surface atoms, and (c) spin-orbit couplings between the $\mathrm{T}$ and $\mathrm{M} d$-bands

When the metal $\mathrm{M}$ forms part of a magnetic nanoparticle covering it, or intrinsically as an alloy, one or more of the three mechanisms invoked to explain magnetic induction of magnetism in $\mathrm{M}$ is also active and magnetism in the $\mathrm{M}$ atom is encountered. Such a study on Co nanoparticles capped with $\mathrm{M}=\mathrm{Ag}, \mathrm{Au}, \mathrm{Pd}$ and $\mathrm{Pt}$ has been performed, as described for the case of Co-Pt NPs in chapter 4. In the Ag and $\mathrm{Au}$ cases the Co nanoparticle remains in its integrity and $M$ covers, without alloying, the Co nanoparticle. The effect of the metal capping is, thus, embedding the Co particle in an M matrix. The net effect is the increase of surface anisotropy at the Co nanoparticle surface atoms $[11,19\rfloor$. In contrast, Pt alloys with Co forming particles with high anisotropy (see chapter 4), and Pd behaves likewise.

In this section, the XMCD results obtained at the $L_{2,3}$ edges of the corresponding capping layer for particles with the same average diameter of $\langle D\rangle \approx 3$ $\mathrm{nm}$ are compared. The data have been obtained at the ID12 beamline, at the ESRF, and the detection technique was fluorescence in backscattering geometry. The polarization was over $90 \%$ in the $\mathrm{Au}$ and $\mathrm{Pt}$ cases, but in the $\mathrm{Ag}$ and $\mathrm{Pd}$ it was 36 and $22 \%$ at the $L_{2}$ edge and 24 and $12.6 \%$, at the $L_{3}$ edge, respectively. The field was applied with an incident angle of $75^{\circ}$ with respect to the normal to the plate. The applied field was $H=10-20 \mathrm{kOe}$ and the temperature $T=5-10 \mathrm{~K}$, thus guarantying the magnetic saturation of the NPs magnetization. The XANES and XMCD results for the four metalcapped Co-NPs, namely, Co-Ag, Co-Au, Co-Pd and Co-Pt NPs are shown in Figs. 5.1 and 5.2. Co-Ag and Co-Au figures are taken from Ref. [11]. The XMCD measurements for Co-Pt NPs are those described in section 4.3.3. Results from the sum rules analysis applied to the XMCD signals in Figs. 5.1 and 5.2 are collected in table 5.2 .

In all cases the non-zero XMCD signal indicates that the ferromagnetic Co has polarized the $\mathrm{M}$ atoms with the total moment parallel to the Co magnetization direction. The Pauli paramagnetic contribution, calculated from table 5.1 data for the applied field, falls within the experimental error bars in the four cases. The $m_{\mathrm{L}} / m_{\mathrm{S}}$ ratio has reduced respect to the bulk metal (compare with table 5.1), with the exception of the bulk Ag, where there is no data. In the case of $\mathrm{Ag}$ and, even more, in $\mathrm{Pd}$, the orbital moment has been practically quenched, while it is at least one order of magnitude larger in 
Table 5.2: Collected data for the M-capped Co NPs with $\langle D\rangle \approx 3 \mathrm{~nm}$. $\xi_{d}$, spin-orbit constant at the $\mathrm{n} d$ orbital; $n_{h}^{d}$, number of holes at the $d$-band; $m_{\mathrm{L}} / m_{\mathrm{S}}$ ratio, $m_{\mathrm{L}}$, orbital moment, $m_{\mathrm{S}}$, spin moment, $m_{\mathrm{tot}}=m_{\mathrm{L}}+m_{\mathrm{S}}$, obtained from the XMCD $L_{2,3}$ edges analysis.

\begin{tabular}{ccccc}
\hline \hline Capping metal & $\mathrm{Ag}^{\mathrm{b}}$ & $\mathrm{Au}^{\mathrm{b}}$ & $\mathrm{Pd}$ & $\mathrm{Pt}$ \\
\hline$\xi_{d}(\mathrm{meV})^{\mathrm{a}}$ & 272 & 608 & 200 & 600 \\
$n_{h}^{d}$ & 0.422 & 0.688 & $1.2^{\mathrm{c}}$ & $1.80(2)$ \\
$m_{\mathrm{L}} / m_{\mathrm{S}}$ & $0.045(5)$ & $0.10(5)$ & $-0.006(3)$ & $0.19(1)$ \\
$m_{\mathrm{L}}\left(\mu_{B}\right)$ & $7(1) \times 10^{-4}$ & $1.5(5) \times 10^{-3}$ & $-0.003(3)$ & $0.027(1)$ \\
$m_{\mathrm{S}}\left(\mu_{B}\right)$ & $0.014(3)$ & 0.015 & $0.45(9)$ & $0.14(1)$ \\
$m_{\text {tot }}\left(\mu_{B}\right)$ & $0.015(3)$ & $0.016(1)$ & $0.45(9)$ & $0.16(1)$ \\
\hline \hline
\end{tabular}

${ }^{a}$ See Ref. [113]. ${ }^{b}$ See Ref. [11.. ${ }^{c}$ See Ref. [165].

the $\mathrm{Au}$ and $\mathrm{Pt}$ cases. This feature is probably related with the fact that the spin orbit coupling constant $\xi_{d}$ is larger in the $\mathrm{Au}$ and $\mathrm{Pt}$ cases (see table 5.2). The $m_{\text {tot }}$ is one order of magnitude smaller in $\mathrm{Ag}$ and $\mathrm{Au}$, with respect to $\mathrm{Pd}$ and $\mathrm{Pt}$, which is related with the much larger $n_{h}^{d}$ in the two latter cases. On the other hand, $m_{\text {tot }}$ is thrice as large in Pd with respect to Pt. This trend is similar to that found for the Pauli paramagnetism, and cannot be directly related to the $n_{h}^{d}$ variation, but rather to a larger exchange enhancement of the paramagnetism in the $\mathrm{Pd}$ case.

\subsection{Intrinsic magnetic moment in $M$ nanoparticles}

Here, the focus is on NPs formed by clusters of M atoms, and show single particle magnetization. There are two distinct, reported, magnetic behaviors, Langevin superparamagnetism (SP)

$$
M(H)=m_{\text {Part }}\left(\operatorname{coth}\left(\frac{m_{\text {Part }} H}{k_{B} T}\right)-\frac{k_{B} T}{m_{\text {Part }} H}\right)
$$

with a single particle moment $m_{\text {Part }}$, and temperature independent magnetism up to room temperature (TI), which may appear simultaneously or independently. Many of the reports deal with conventional magnetometric data, and doubts are cast on the influence of spurious contributions. Our approach to this problem has been to restrict ourselves to the XMCD available data because of its element selectivity that allows to discriminate the magnetization originating from the actual $\mathrm{M}$ metal. As in the previous sections, the $\mathrm{M}$ $L_{2,3}$ edges inform on the contribution of the $n d$-band to this NPs magnetism.

It has been reported that Ag NPs of $\langle D\rangle=2.3 \mathrm{~nm}$, capped with do- 

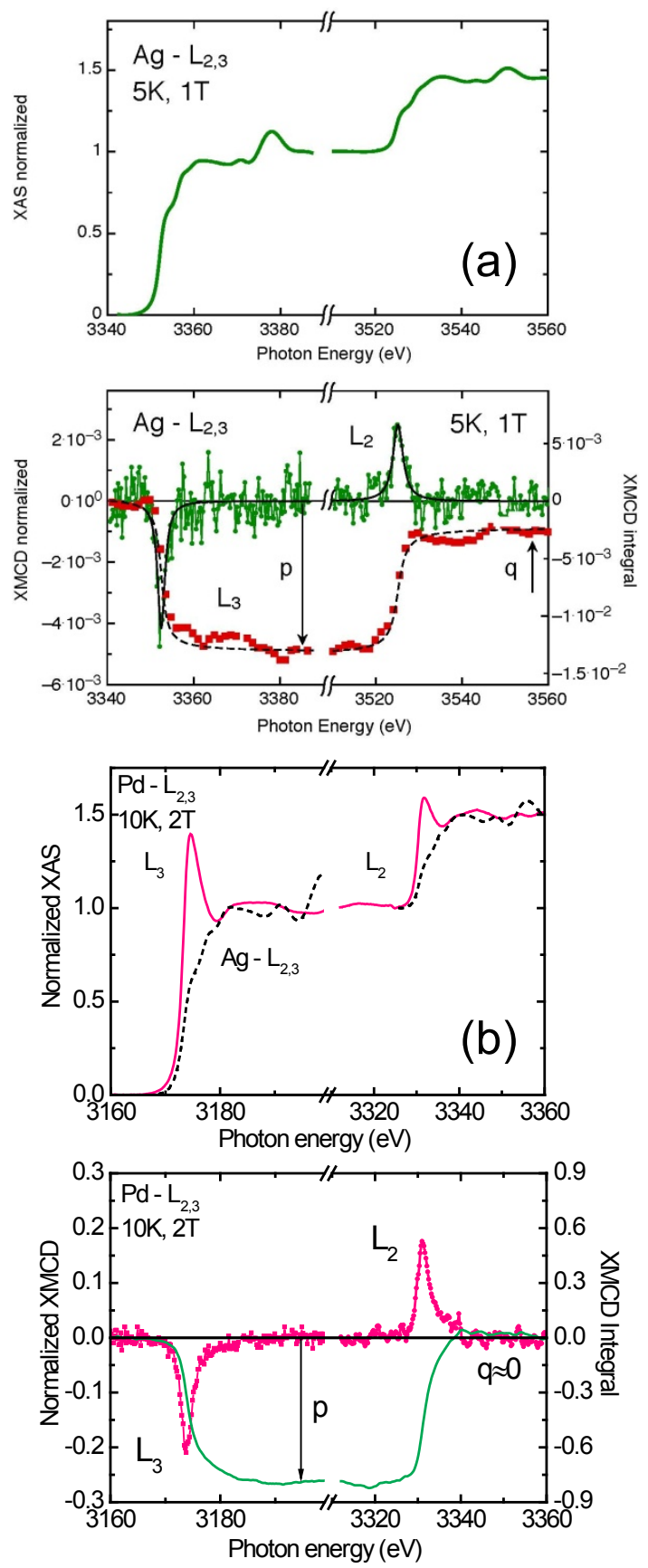

Figure 5.1: Normalized XANES and XMCD at the M $L_{2,3}$ edges in the Co-M NPs. (a) Co-Ag NPs $\left\lfloor 11\right.$, and (b) Co-Pd NPs. The XANES spectra of Ag $L_{2,3}$ edges are shown in (b) for comparison. The higher limit for the $\mathrm{p}$ and $\mathrm{q}$ integrals, used for the sum rules analysis, is given. 

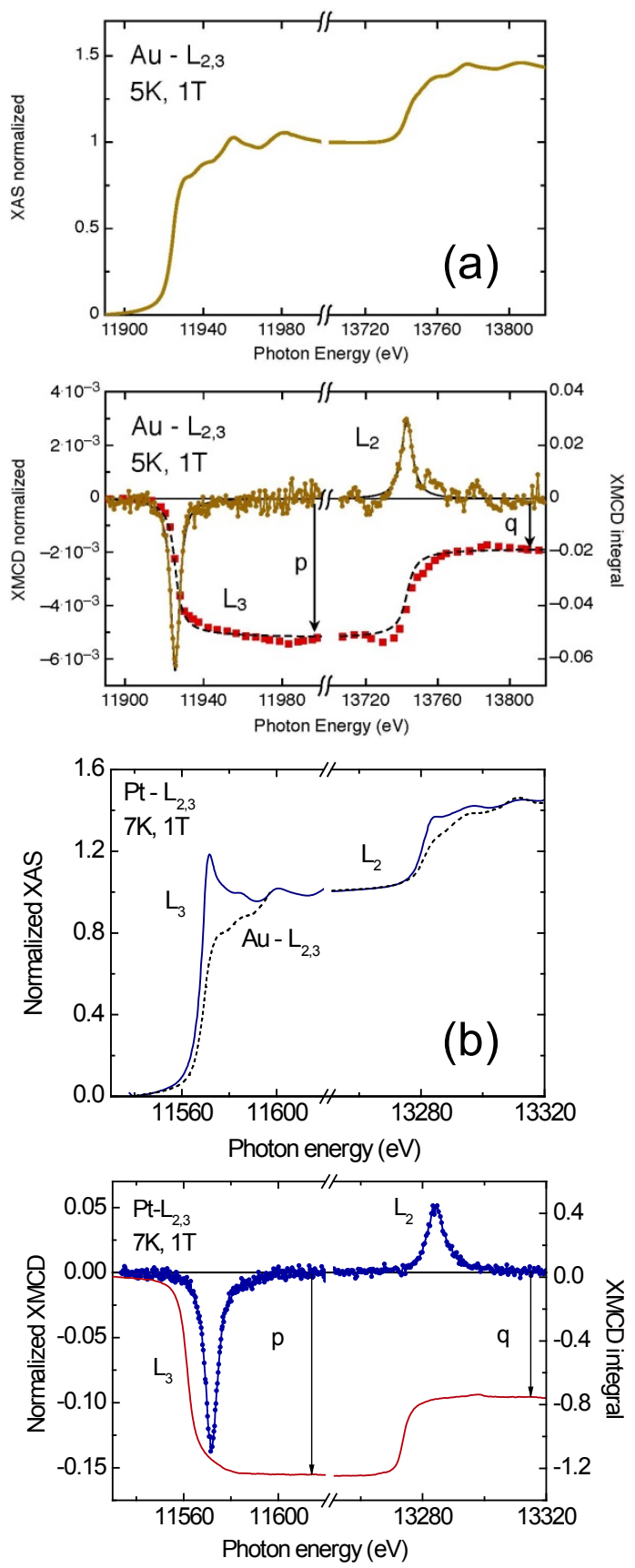

Figure 5.2: Normalized XANES and XMCD at the M $L_{2,3}$ edges in the Co-M NPs. (a) Co-Au NPs 11$\rfloor$ and (b) Co-Pt NPs. The XANES spectra of Au $L_{2,3}$ edges are shown in (b) for comparison. The higher limit for the $\mathrm{p}$ and $\mathrm{q}$ integrals, used for the sum rules analysis, is given. 
decanelthiol molecules, show a spontaneous, constant moment up to room temperature. However, there are no XMCD experiments yet done on these particles $\lfloor 168,169\rfloor$.

Although $\mathrm{Au}$ NPs have been extensively investigated with conventional magnetometry (SQUID), relatively few experiments have been done with XMCD. The first of these experiments detected a Langevin paramagnetism and a small TI contribution in $\langle D\rangle=1.9 \mathrm{~nm}$ Au NPs capped with polyallyl amine hydrochloride (PAAHC). The NP magnetic moment was determined to be $m_{\text {Part }}=0.4 \mu_{B}$. The TI component is quite sizeable and both SQUID and XMCD data coincide below $T=100 \mathrm{~K}$. The XMCD signal is very small $\left(10^{-5}\right.$ $\mathrm{XMCD} / \mathrm{XAS}$ ratio at the $L_{3}$ edge) (see table 5.3) 1170 . The second report concerned $\mathrm{Au}\langle D\rangle=2 \mathrm{~nm}$ NPs capped with dodecanethiol, where the XMCD signal was similar to that of the PAAHC capped Au NP. Again, it yields to a very minute XMCD signal $\left(10^{-5} \mathrm{XMCD} / \mathrm{XAS}\right.$ ratio) [169]. However, this small XMCD signal is assigned to the surface atoms only, and the authors propose $m_{\mathrm{at}}=0.33 \mu_{B} /$ magnetic Au atom. Finally, Au NPs capped with thiol and embedded in polyethylene were measured with XMCD [171」, finding also a very small XMCD signal as compared to the TI component.

Our contribution to this subject has been the detection of a 25 times larger XMCD signal, shown in Fig. 5.3(a), in NPs of similar size deposited on a template of the Sulfolobus acidocaldarius S-layer [35]. $m_{\text {Part }}$ has been estimated to be of several Bohr magnetons. As in the previous cases, the presence of sulfur $(\mathrm{S})$ atoms at the template surface binding the Au particle seems to be of paramount importance to show this type of magnetism, since it does not appear on Au particles deposited on Bacilus Sphericus S-layer, where there is no $\mathrm{S}$ present. We demonstrate that on each Au particle the thiols and other groups present in the S-layer supply holes to the $5 d$ band. Their spins are ferromagnetically coupled by a spin-spin exchange interactions. In addition, an appreciable orbital moment is generated by spin-orbit couplings. Hund's third rule is complied and the orbital moment is parallel to the spins. The magnetic behavior of the particle is paramagnetic, with very low anisotropy. Thus, the external field sets the common alignment direction of the magnetization of all particles. The very large magnetic moment per Au atom observed in the XMCD measurements originates from the large charge transfer from the S-layer supporting template to the Au surface atoms, and reshuffling of holes to the particle core. Finally, we may also remark that the striking magnetic properties of these Au NPs are due to the special synthesis on a unique biological matrix containing low number of thiol groups distributed in a highly ordered way $\lfloor 172\rfloor$.

As in the case of the $\mathrm{Ag}$ case, $\mathrm{Pd}$ NPs have been reported to show a 

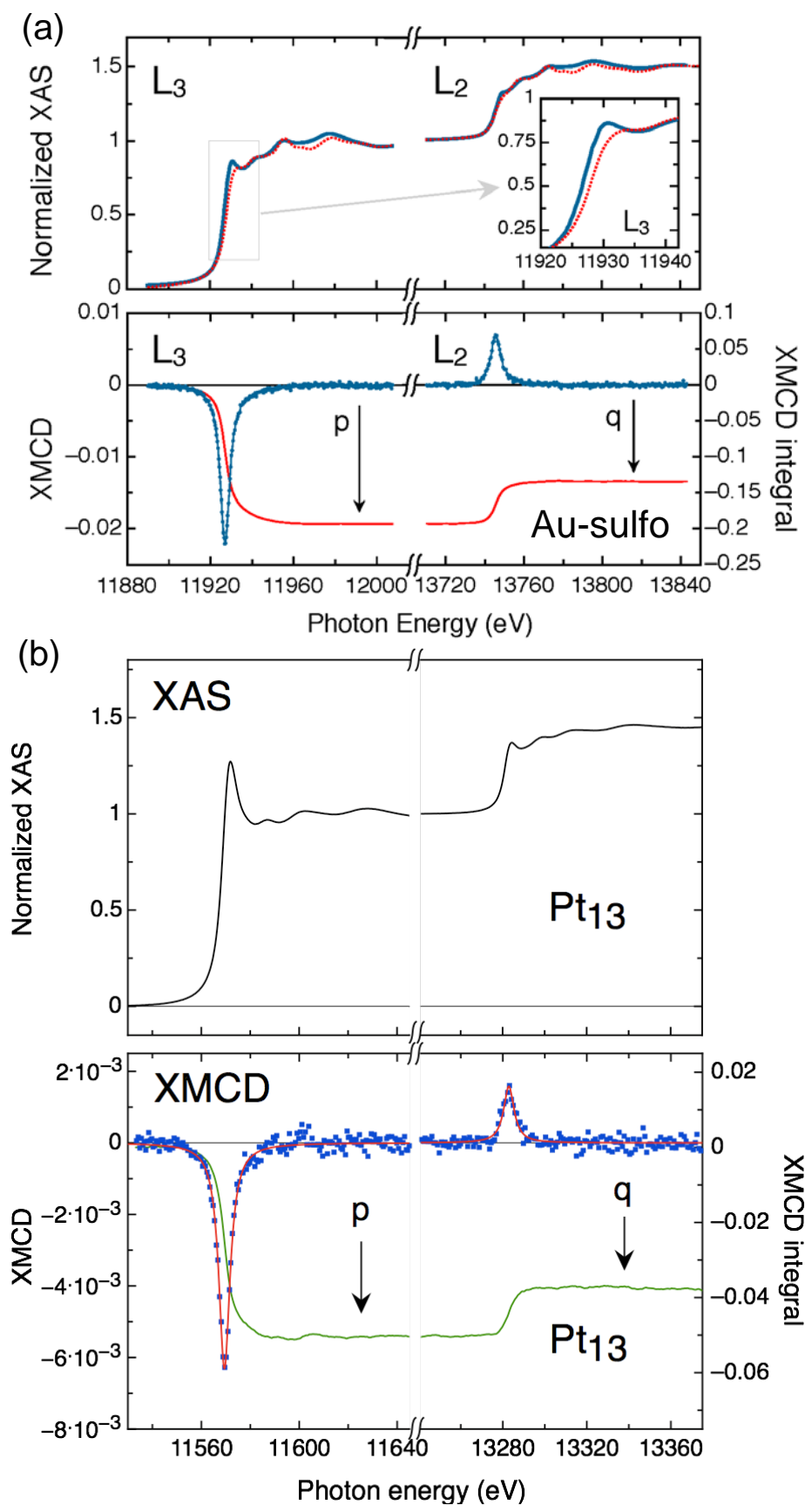

Figure 5.3: Normalized XANES and XMCD at the M $L_{2,3}$ edges in the (a) Au NPs $\lfloor 172$. and (b) Pt NPs $\lfloor 34$. The higher limit for the $\mathrm{p}$ and $\mathrm{q}$ integrals, used for the sum rules analysis, is given. 
Table 5.3: Collected data for the M NPs studied with XMCD. $\langle D\rangle$, particle diameter; $n_{h}^{d}$, number of holes at the $d$-band; $m_{\mathrm{L}} / m_{\mathrm{S}}$ ratio, $m_{\mathrm{L}}$, orbital moment, $m_{\mathrm{S}}$, spin moment, $m_{\mathrm{at}}=m_{\mathrm{L}}+m_{\mathrm{S}}$, obtained from the XMCD $L_{2,3}$ edges analysis; $m_{\text {Part }}$, the NP magnetic moment deduced from the Langevin curve.

\begin{tabular}{|c|c|c|c|c|}
\hline & \multicolumn{3}{|c|}{$\mathrm{Au} \mathrm{NPs}$} & \multirow{2}{*}{ Pt NPs ${ }^{d}$} \\
\hline & $\mathrm{PAAHC}^{\mathrm{a}}$ & $\mathrm{DT}^{\mathrm{b}}$ & Sulfolobus & \\
\hline Type & $\mathrm{SP}$ & $\mathrm{TI}$ & $\mathrm{SP}$ & $\mathrm{SP}$ \\
\hline$\langle D\rangle(\mathrm{nm})$ & 1.9 & 2 & $2.6(1)$ & 0.7 \\
\hline$n_{h}^{d}$ & & & 0.762 & 1.85 \\
\hline$m_{\mathrm{L}} / m_{\mathrm{S}}$ & 0.145 & 0.10 & $0.28(1)$ & $0.32(2)$ \\
\hline$m_{\mathrm{L}}\left(\mu_{B} / \mathrm{at}\right)$ & & & $0.0110(1)$ & $0.0055(1)$ \\
\hline$m_{\mathrm{S}}\left(\mu_{B} / \mathrm{at}\right)$ & & & $0.039(1)$ & $0.0171(6)$ \\
\hline$m_{\text {tot }}\left(\mu_{B} /\right.$ at $)$ & 0.001 & 0.33 & $0.050(1)$ & $0.0226(9)$ \\
\hline$m_{\text {Part }}\left(\mu_{B}\right)$ & 0.4 & & 2.3 & $3.5-4.9$ \\
\hline
\end{tabular}

magnetic behavior due to size-effect and, or, surface atoms polarization [173], however, there is no confirmation by XMCD of this feature.

Finally, $\mathrm{Pt}_{13}$ nanoparticles embedded in a $\mathrm{NaY}$ zeolite matrix have also shown superparamagnetic behavior at low temperature and high field, as observed first with SQUID magnetometry [174]. The $\mathrm{Pt}_{13}$ particle crystallizes in icosahedral symmetry and have 12 of its atoms at the surface, thus size and surface effect are maximized. The XMCD measurement performed at the Pt $L_{2,3}$ edges at $T=7 \mathrm{~K}$ and increasing field up to $H=60 \mathrm{kOe}$ gave an unambiguous non-zero signal, as shown in Fig. 5.3(b), which proved the existence of intrinsic magnetization in these particles. The $m_{\text {Part }}$ deduced from the Langevin curve ranges between 3.5 and $4.9 \mu_{B}$, and the moment per $\mathrm{Pt}$ atom deduced from the experiment is too low to account for all existent particles to be magnetic, actually, only $12 \%$ seem to have this property. It may be due to the different chemical surroundings in the zeolite cavity where the cluster sits $[34\rfloor$.

Comparing the results in table 5.3 , the ratio $m_{\mathrm{L}} / m_{\mathrm{S}}$ of $\mathrm{Au}$ NPs varies strongly depending on the capping material, but in two out of three cases, it is larger than in the induced magnetic case of Co-Au capping (see tables 5.2 and 5.3). The value in the case of $\mathrm{Pt}_{13}$ is larger than in the Co-Pt alloyed NPs (see tables 5.2 and 5.3). In both cases, $\mathrm{Au}$ and Pt NPs, there is an increase in the number of holes $n_{h}^{d}$ with respect to the corresponding bulk metal. This seems to be a necessary condition for $d$-band magnetism of intrinsic type. 


\subsection{Conclusions}

XMCD has been able to establish that there is $d$-band magnetism in the metallic Ag, Au, Pd and Pt at the nanoscale. Besides, it has shown that about $30 \%$ of this magnetism is orbital in its origin. However, this technique gives, inherently, just an average value of the $m_{\mathrm{L}}, m_{\mathrm{S}}$ components over all $\mathrm{M}$ atoms present in the sample. Therefore, it is unable to discriminate whether the magnetism originates at the surface (by chemical bonding, symmetry breaking, or both), or in the core of the particle. In our opinion, at present this is the most intriguing question, there being arguments and theories in both directions $\lfloor 175\rfloor$. These are, among others, some of the open questions in the fascinating realm of the magnetism of metals. 



\section{Chapter 6}

\section{Transverse susceptibility measurement system for the PPMS}

This chapter is dedicated to describe the magnetic transverse susceptibility (TS) technique: a measurement method, hardly used, but very effective, to directly probe the magnetic anisotropy of interesting materials, especially of nanoparticulate systems. A TS measurement system based on a simple inverter CMOS cell oscillator cross-coupled to a $L C$ tank is presented, which we have implemented to operate at a Quantum Design Physical Properties Measurement System (PPMS) at the Servicio de Medidas Físicas of the Universidad de Zaragoza. ${ }^{1}$ This implementation has been performed in collaboration with people from the Servicio de Medidas Físicas and from the Servicio de Instrumentación Electrónica of the Universidad de Zaragoza. ${ }^{2}$ It has also been possible thanks to the advise of Dr. Hariharan Srikanth and members of the Functional Materials Laboratory at the University of South Florida in Tampa, FL, USA. ${ }^{3}$

We introduce several improvements with respect to similar currently operating TS measurement equipments [51]. The electronics have been redesigned to use CMOS transistors as active devices, which simplifies the circuit design and enlarge the tuning range, thus making the proposed electronic block more feasible, predictable, and precise. Additionally, we propose a newly designed sample holder, which facilitates the procedure to change a sample and improves reproducibility of the circuit. Our design minimizes the thermal leak of the

\footnotetext{
${ }^{1}$ http://sai.unizar.es/medidas/index.html

${ }^{2}$ http://sai.unizar.es/electronica/index.html

${ }^{3}$ http://shell.cas.usf .edu/fml/
} 
measuring probe by one order of magnitude, allowing to measure from $1.8 \mathrm{~K}$ in standard PPMS systems, thanks to the use of a low temperature Beryllium Copper coax cable instead of the conventional RG402 Cu coax cable employed in the insert for the PPMS in similar systems. The data acquisition method is also simplified, so that the measuring sequences are implemented directly in the PPMS controller computer by programming them in the Quantum Design MultiVu software that controls the PPMS. We present the test measurements performed on the system without sample to study the background signal and stability of the circuit. Measurements on a $\mathrm{Gd}_{2} \mathrm{O}_{3}$ calibrating sample yield to the estimation of the system sensitivity, which is found to be on the order of $10^{-6}$ emu. Finally, measurements on a $\mathrm{TmCo}_{2}$ Laves phase sample with a ferrimagnetic transition temperature around $4 \mathrm{~K}$ are described, demonstrating that the developed system is well suited to explore interesting magnetic phenomena at this temperature scale.

\subsection{Theoretical model of the magnetic transverse susceptibility}

A transverse susceptibility measurement consists in probing the ac magnetic susceptibility of a material in one direction while an external bias magnetic field is applied perpendicular to the measurement direction. Analysis of the TS in magnetic materials have demonstrated its potential and versatility to study singular magnetic properties of bulk $[36-38$, single crystals $[39\rfloor$, thin films $\lfloor 40-43\rfloor$ and nanoparticles (NPs) systems $\lfloor 44-48,176\rfloor$.

The first coherent theoretical approach for TS was proposed by Aharoni

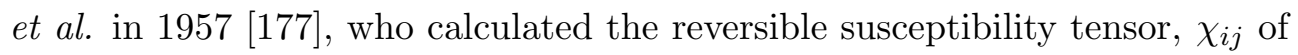
a Stoner-Wohlfarth (SW) particle [4]. The susceptibility tensor is defined as $\chi_{i j}=d M_{i} / d H_{j}$ and its diagonal components comprise the longitudinal susceptibility component, $\chi_{\mathrm{L}}$, measured in the main field bias direction, and the two transverse susceptibility components $\chi_{\mathrm{T} 1}$ and $\chi_{\mathrm{T} 2}$, measured in directions perpendicular to the bias field.

Lets analyze the case of a SW particle, i.e., an ellipsoid, uniaxial single domain, ferromagnetic particle, with volume $V$, anisotropy constant $K_{1}$ and saturation magnetization, $M_{S}$, as the one shown in Fig. 6.1(a). The bias field, $H_{\mathrm{DC}}$, is applied along the $z$ axis, and the perturbing radio frequency (rf) magnetic field, $H_{\mathrm{rf}}$, along the $x$ axis. In this case, the longitudinal susceptibility is $\chi_{\mathrm{L}}=\chi_{z z}=d M_{z} / d H_{z}$, and the transverse susceptibility components $\chi_{\mathrm{T} 1}=\chi_{x x}=d M_{x} / d H_{x}$ and $\chi_{\mathrm{T} 2}=\chi_{y y}=d M_{y} / d H_{y} . \quad M_{i}$ and $H_{i}$ are the projections of the vectors saturation magnetization, $\vec{M}_{\mathrm{S}}$, and total magnetic 

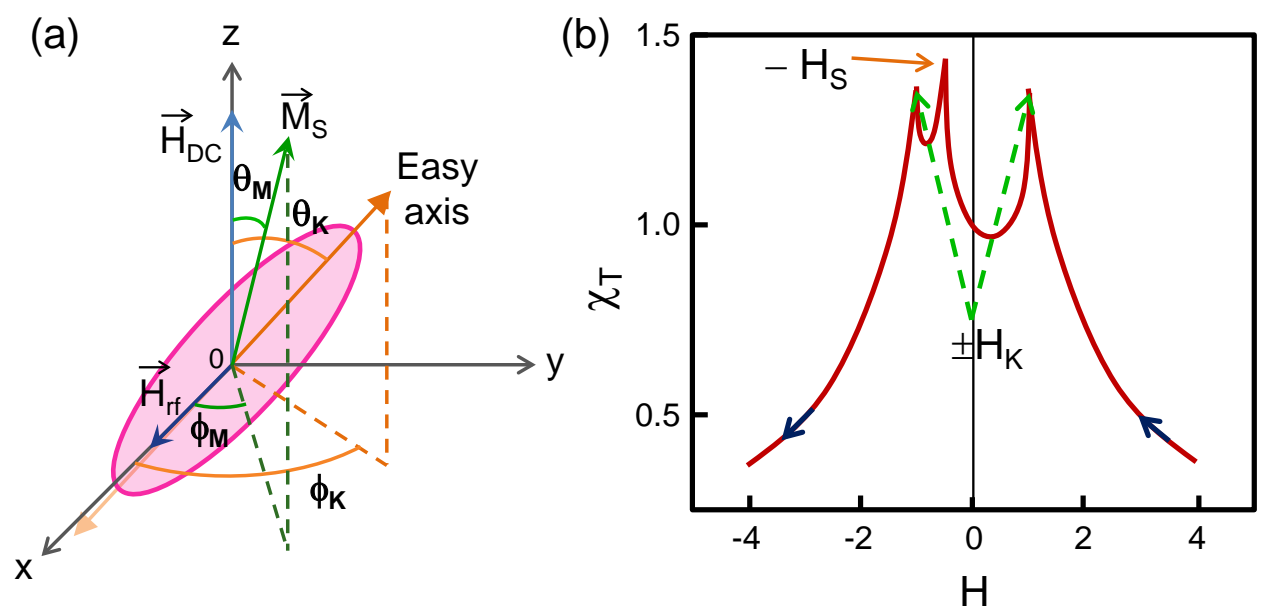

Figure 6.1: (a) Diagram of a TS measurement geometry of a uniaxial magnetic particle 48. (b) Typical TS unipolar curve as a function of $H_{\mathrm{DC}}$ for a collection of randomly oriented particles.

field, $\vec{H}_{\mathrm{DC}}=\vec{H}_{\mathrm{DC}}+\vec{H}_{\mathrm{rf}}$, respectively.

The energy of the SW particle is given by the sum of the anisotropy energy, $E_{K}$, and the Zeeman energy, $E_{H}$, the latter defining the interaction with the applied field. Based on the coordinate system given in Fig. 6.1(a), the total energy may be written as:

$$
E=-K_{1}\left(\vec{M}_{\mathrm{S}} \cdot \vec{u}_{K}\right)^{2}-\vec{M}_{\mathrm{S}} \cdot\left(\vec{H}_{\mathrm{DC}}+\vec{H}_{\mathrm{rf}}\right)
$$

The vector $\vec{u}_{K}$ is the unit vector in the easy axis direction. The torques caused by the anisotropy and $H_{\mathrm{DC}}$ will determine the equilibrium position of the particle magnetic moment $\left(\theta_{M}, \phi_{M}\right)$ while the small alternating field, $H_{\mathrm{rf}}$, will produce only small perturbations around this equilibrium position. From the point view of the energy of the magnetic system, the stable equilibrium positions of magnetization vector are given by the conditions for minimum of the total energy, this is

$$
\frac{\partial E}{\partial \theta_{M}}=0 ; \quad \frac{\partial^{2} E}{\partial \theta_{M}^{2}} \geq 0 \quad \text { and } \quad \frac{\partial E}{\partial \phi_{M}}=0 ; \quad \frac{\partial^{2} E}{\partial \phi_{M}^{2}} \geq 0 ;
$$

Then, taking into account these conditions, and minimizing equation 6.1, one obtains the following expression for the transverse susceptibility, $\chi_{\mathrm{T} 1}$ as an example 


$$
\begin{aligned}
\chi_{\mathrm{T} 1}=\frac{3}{2} \chi_{0} & \left(\cos ^{2} \phi_{K} \frac{\cos ^{2} \theta_{M}}{h \cos \theta_{M}+\cos 2\left(\theta_{M}+\theta_{K}\right)}\right. \\
& \left.+\sin ^{2} \phi_{K} \frac{\sin \left(\theta_{K}-\theta_{M}\right)}{h \sin \theta_{K}}\right)
\end{aligned}
$$

where, $\chi_{0}=M_{\mathrm{S}}^{2} / 3 K_{1}, h$, the reduced field defined as $h=H_{\mathrm{K}} / H_{\mathrm{DC}}$, and $H_{\mathrm{K}}=2 K_{1} / M_{\mathrm{S}}$ corresponds to the anisotropy field.

For an array of particles with randomly oriented anisotropy axes, assuming the particles are identical and that inter-particle interactions are negligible, the average $\chi_{\mathrm{T}}$ becomes

$$
\left\langle\chi_{T}\right\rangle=\frac{1}{2 \pi} \int_{0}^{2 \pi} \int_{0}^{\pi / 2} \chi_{\mathrm{T}} \sin \theta_{K} d \theta_{K} d \phi_{K}
$$

after integrating over $\phi_{K}$ and substituting back equation 6.3 , we arrive at the expression

$$
\begin{aligned}
\left\langle\chi_{\mathrm{T}}\right\rangle=\frac{3}{2} \chi_{0} \int_{0}^{\pi / 2} & {\left[\frac{\cos ^{2} \theta_{M}}{h \cos \theta_{M}+\cos 2\left(\theta_{K}-\theta_{M}\right)}\right.} \\
& \left.+\frac{\sin \left(\theta_{K}-\theta_{M}\right)}{h \sin \theta}\right] \sin \theta_{K} d \theta_{K}
\end{aligned}
$$

The transverse susceptibility curve for this system, as a function of the bias field, is plotted in Fig. 6.1(b). It shows three cusps: two of them located at $\pm H_{\mathrm{K}}$, and the third one at the switching field $H_{\mathrm{S}}$.

\subsection{Description of the TS measurement system}

The first experimental confirmation of Aharoni's theory for TS was performed in 1987 by Pareti and Turilli [49], who showed the presence of $\pm H_{\mathrm{K}}$ and $H_{\mathrm{S}}$ peaks on barium ferrite particles. Their TS measurement system was based on conventional inductance susceptibility techniques, method also used by other groups $\lfloor 46,50\rfloor$. In addition to that system, TS measurement techniques developed up to date are based on magneto-optical setups $\lfloor 40,43\rfloor$ and self-resonant $L C$ tank circuits in the radio frequency range $[51]$. The latter technique has demonstrated to be one of the most accurate and versatile developments to measure TS, since it may be implemented on widely used commercial Quantum Design physical properties measurement systems (PPMS), as illustrated 


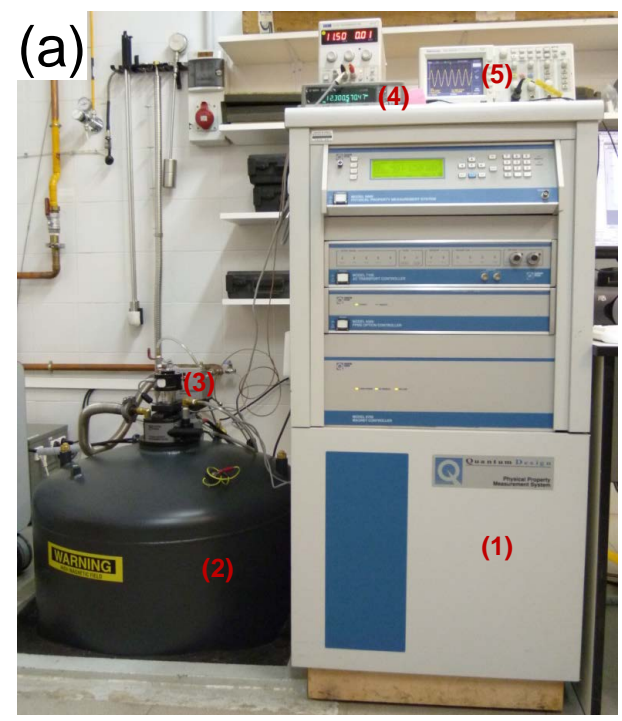

(b)

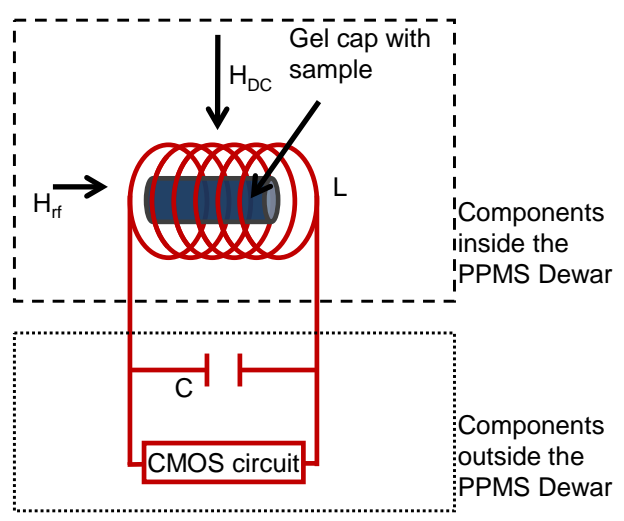

Figure 6.2: (a) Picture of the PPMS with the TS setup: (1) Model 6000 PPMS control, (2) dewar, (3) multi functional probe's head, (4) frequency counter and (5) oscilloscope. (b) Diagram of the TS measurement system.

in Fig. 6.2. The tank inductance, $L$, is provided by a pick-up coil located in a controlled temperature and magnetic field environment. The capacitor, $C$, is usually fixed at room temperature outside the insert. The standard expression to define the resonance frequency of the tank is

$$
f=\frac{1}{2 \pi \sqrt{L C}}
$$

In these kind of setups, the magnetic sample is inserted into a gel cap that snugly fits into the inductive coil, and $\Delta f$ is measured as an external static field $\left(H_{\mathrm{DC}}\right)$ or temperature are varied. Changes in $f$ are direct consequence of changes in $L$, described by the expression

$$
\frac{\Delta f}{f} \approx-\frac{\Delta L}{2 L}
$$

obtained after differentiating equation 6.6.

The total inductance of the coil with the sample inside, $L$, is defined in terms of the empty coil inductance, $L_{0}$, and the contribution of the sample, $L_{\mathrm{S}}$. The latter depends on the permeability of the sample, $\mu=\mu_{0}(1+\chi)$, where $\chi$ is its volume magnetic susceptibility (dimensionless). Then, considering the geometry of the coil, i.e., its volume, $V_{0}$, its length, $l$, and the number of turns, $N$, and the volume of the sample inside it, $V_{\mathrm{S}}$, the total inductance $L$ 
is expressed as

$$
L=\mu_{0}\left(\frac{N}{l}\right)^{2}\left(V_{0}+\chi V_{\mathrm{S}}\right)
$$

The change in inductance $\Delta L$, calculated after differentiating equation 6.8 , is written as

$$
\Delta L=\mu_{0}\left(\frac{N}{l}\right)^{2} \Delta \chi V_{\mathrm{S}}
$$

Since we want to express $\Delta L / L$, we divide equation 6.9 by $L$ obtaining

$$
\frac{\Delta L}{L}=\frac{\Delta \chi V_{\mathrm{S}}}{V_{0}+\chi V_{\mathrm{S}}}
$$

Two approximations may be derived from this expression. On one hand, if $\chi V_{\mathrm{S}} \gg V_{0}$, equation 6.10 is reduced to

$$
\frac{\Delta L}{L} \approx \frac{\Delta \chi}{\chi}
$$

On the other hand, if $\chi V_{\mathrm{S}} \ll V_{0}$, equation 6.10 may be written as

$$
\frac{\Delta L}{L} \approx \Delta \chi \eta
$$

where $\eta=V_{\mathrm{s}} / V_{0}$ represents the fill factor of the coil core space.

When the geometry of the measurement setup is that illustrated in Fig. $6.2(\mathrm{~b})$, where the perturbing rf magnetic field inside the coil $\left(H_{\mathrm{rf}} \sim 10 \mathrm{Oe}\right)$ is oriented perpendicular to the bias field, $H_{\mathrm{DC}}$, the magnetic susceptibility of the sample being probed is its transverse component $\left.\chi_{\mathrm{T}} \mid 51\right\rfloor$, as described in section 6.1. Therefore, combining equations 6.7 and 6.11 , we arrive at the direct relation between $\Delta f$ and $\Delta \chi_{\mathrm{T}}$, as

$$
\frac{\Delta f}{f} \approx-\frac{1}{2} \frac{\Delta \chi_{\mathrm{T}}}{\chi_{\mathrm{T}}}
$$

and from equations 6.7 and 6.12 we get

$$
\frac{\Delta f}{f} \approx-\frac{1}{2} \Delta \chi_{\mathrm{T}} \eta
$$




\subsection{Design and implementation of the oscillator cir- cuit}

TS magnetometry equipments based on self-resonant systems usually employ a tunnel diode oscillator (TDO) as their active device in the radio frequency resonator setup [51]. However, there are several important drawbacks of using TDOs for this application, which may be summarized as: 1) they are complex devices with low market availability and 2) the tuning range for accomplishing the oscillation condition is reduced, thus limiting the nature of the measurable magnetic samples. Instead, the proposed oscillator uses CMOS transistors as active devices, implementing a simple inverter cell in cross-coupled topology, which compensates the energy loss of the $L C$ resonant tank. The choice of these devices and configuration not only solve the above mentioned TDO drawbacks, but also involve some other benefits like: 1) minimum parasitics and noise sources increasing the sensitivity and accuracy of the resonant circuit, 2) higher circuit robustness and predictability, giving a deep understanding about the block properties, and 3) much lower cost in the future case of high-volume manufacturing.

Figure 6.3 shows the electronic schematic of the complete circuit, where the described oscillator is denoted in the (1) zone. The theoretical basis of the oscillator design is detailed in Ref. [178]. The main aspects of the cell modeling are described as follows. The small-signal equivalent model of the proposed oscillator is illustrated in Fig. 6.4(a). The $L C$ tank is represented by the impedance $Z_{L}$. Applying conventional circuit theory, the characteristic equation of this model is obtained as a function of the $\mathrm{Z}_{L}$ transfer function,

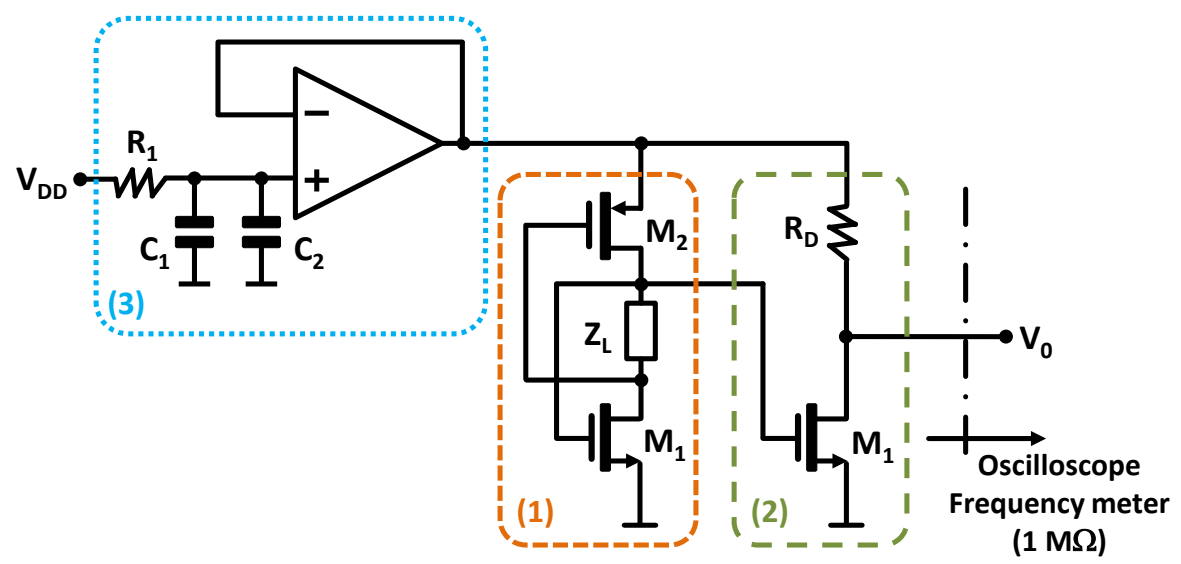

Figure 6.3: Electronic schematic of the CMOS based $L C$ cross-coupled oscillator circuit used for TS measurements. 
as

$$
A-B Z_{L}=0
$$

where,

$$
\begin{gathered}
A=r_{01}+r_{02}+r_{01} r_{02}\left(g_{m 1}+g_{m 2}\right) \approx r_{01} r_{02}\left(g_{m 1}+g_{m 2}\right) \\
B=g_{m 1} g_{m 2} r_{01} r_{02}-1 \approx g_{m 1} g_{m 2} r_{01} r_{02}
\end{gathered}
$$

$\mathrm{Z}_{L}$ is modeled as a $L-R-C$ circuit given the inherent internal DC resistance of the inductive coil, as drawn in Fig. 6.4(b). Given this model, the load transfer function, $\mathrm{Z}_{L}$, is expressed as:

$$
Z_{L}(s)=\frac{s L+R}{s^{2} L C+s C R+1}
$$

Substituting equation 6.18 in 6.15 , the final expression of the characteristic equation results in

$$
A L C s^{2}+s(A R C-B L)+(A-B R)=0
$$

Taking into account solutions $s= \pm j \omega$, and equalling the real and imaginary terms to zero, the oscillation frequency and oscillation condition are functions of $L, C$, and $R$, and are expressed as

$$
\begin{gathered}
f^{2}=\frac{1}{(2 \pi)^{2} L C}[1-R \alpha] \\
1 \leq \frac{L \alpha}{R C}
\end{gathered}
$$

where $\alpha=\left(g_{m 1} g_{m 2}\right) /\left(g_{m 1}+g_{m 2}\right)$, and $g_{m 1,2}$ are the characteristic transconductances of the $\mathrm{M}_{1,2}$ transistors shown in Fig. 6.3. These transistors are implemented with a commercial device, ALD1105 IC (two NMOS and two PMOS transistors into a single chip). The use of this standard CMOS chip guarantees high simplicity in modeling the transistors, validating the first order quadratic approximation described in Ref. [179」. This fact is quite important because it minimizes errors when determining the shift in the resonance frequency caused by the change in inductance for the TS calculation. 
(a)

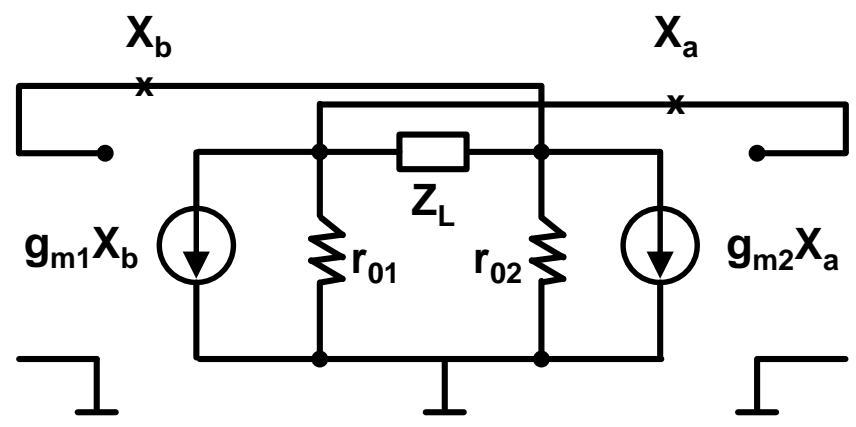

(b)

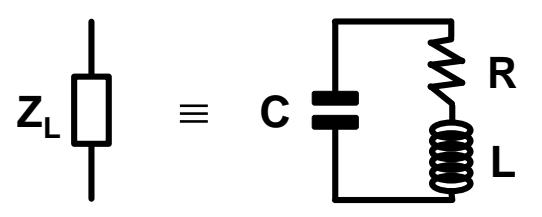

Figure 6.4: (a) Small-signal equivalent model of the proposed oscillator. (b) Realistic model of the $L C$ resonant tank

In addition to the inverter cell and the $L C$ tank, the circuit includes two extra stages. One of them is an output driver working as second oscillator stage. This driver (shown in the (2) marked zone in Fig. 6.3) is made by an NMOS passively loaded common-source stage and isolates the resonator tank from the posterior measurement equipment. This alleviates the load effect, minimizing the electronic impact of the connections to the measurement setup (the oscilloscope and frequency counter that will be described below). The second additional stage (denoted in the (3) area in Fig. 6.3) is a supply isolation block consisting of a voltage amplifier in buffer topology with a $R C$ filtering network. It is crucial to isolate the $L C$ resonant tank since this noise could generate phase noise into the oscillation. Different voltage amplifiers were tested in the design process of this block in order to optimize the stability and working conditions, obtaining the best trade-off with a LF356 OpAmp. Since the designed circuit operates at radio frequencies, the filtering network is based on a $R-2 C$ bypassing filter with values of $3 \Omega, 2.2 \mathrm{nF}$ and $220 \mathrm{nF}$, respectively (see Fig. 6.3, (3) marked area).

The circuit is integrated into the PPMS, using a model P450A - PPMS Multi-Function Probe (MFP) insert, shown in Fig. 6.5(a). The components of the circuit were placed as close as possible (thus minimizing parasitic effects) and mounted on a copper double layer board with glass fiber as isolation material, which is located inside the MFP head (see Fig. 6.5(b) for details of the final board design and Fig. 6.5(c) for the location of the board circuit into the probe's head). The capacitor of the $L C$ tank, $C=22 \mathrm{pF}$, is located on 


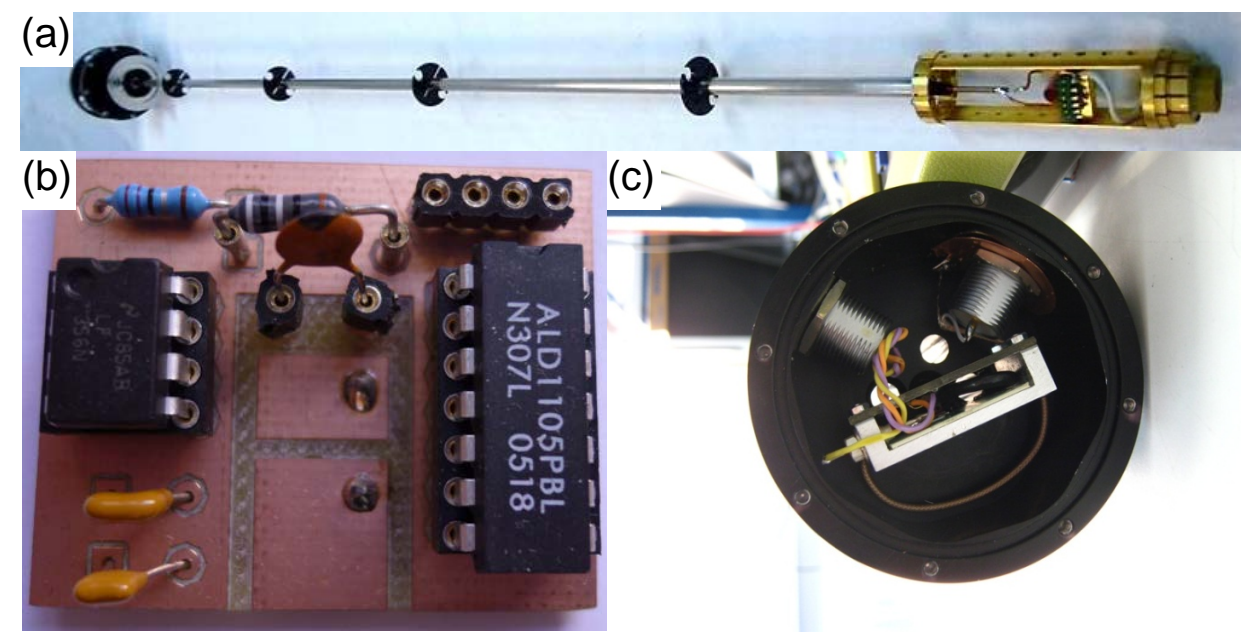

Figure 6.5: (a) MFP used for the TS setup. (b) Details of the circuit board with the CMOS based circuit. (c) Location of the circuit in the MFP's head.

the circuit board, while the inductor is placed at the end of the MFP, i.e., it is inserted into the PPMS dewar since it holds the sample to be measured (see location of the insert in the PPMS dewar in Fig. 6.2(a)). Discrete devices have been employed in this prototype in order to check different frequency ranges and bias conditions. Connections between the circuit board and the coil were made by a $0.9 \mathrm{~m}$ long non-magnetic beryllium copper - $50 \Omega$ semi-rigid coaxial cable, with a 2.19 mm diameter (Coax co. ref. SC-219/50 B-B). The parasitic capacitance of the coaxial is about $86 \mathrm{pF}$, and the self inductance is around $200 \mathrm{mH}$, according to the manufacturer specifications; these two contributions must be taken into account when calculating the nominal resonance frequency of the $L C$ tank.

We have chosen this specific coax to minimize the thermal leak in the probe inside the PPMS dewar at low temperatures, as we demonstrate as follows. The thermal leak introduced by the coaxial cable in the low temperature system can be estimated by calculating the heat transfer rate by thermal conductance $(Q / t)$ of the coax, according to the dimensions and thermal conductivity $(\kappa)$ of the cable. ${ }^{4}$ This thermal leak for the Be-Cu coaxial cable $(\kappa=65.77$ $\mathrm{W} \mathrm{m} \mathrm{m}^{-1} \mathrm{~K}^{-1}$ at $300 \mathrm{~K}$ and cross-sectional area $=1.58 \times 10^{-6} \mathrm{~m}^{2}$ ) is $0.045 \mathrm{~W}$, while the one for a conventional RG402 Cu coaxial used in similar TS setups $\left(\kappa=385.95 \mathrm{~W} \mathrm{~m}^{-1} \mathrm{~K}^{-1}\right.$ at $300 \mathrm{~K}$ and cross-sectional area $\left.=3.07 \times 10^{-6} \mathrm{~m}^{2}\right)$ is $1.23 \mathrm{~W}$. The reduction of the thermal transfer rate by a factor of 27 by using

\footnotetext{
${ }^{4} \mathrm{~A}$ useful tool to calculate the heat transfer by thermal conductance of several materials can be found in the Quantum Design website www.qdusa.com/techsupport/ thermalCalculator.html
} 


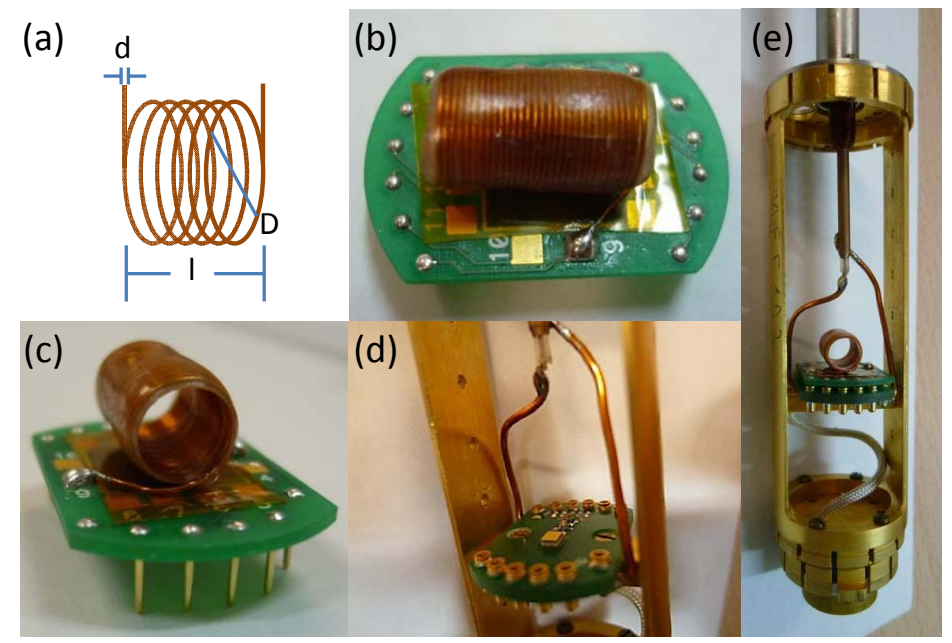

Figure 6.6: (a) Diagram of the coil geometry. (b) and (c) views of the coil assembly on the universal sample board. (d) Close up of the plug-in platform. (e) Final assembly of the coil at the bottom of the MFP.

a $\mathrm{Cu}-\mathrm{Be}$ coax in the setup is what allowed us to reach the lowest temperature possible with a conventional probe insert for the PPMS, i.e., $T_{\text {base }}=1.8 \mathrm{~K}$.

The circuit specifications were optimized to use an inductive air coil, $L$, which is a 31 - turn solenoid hand - wound using a $0.3 \mathrm{~mm}$ in diameter $\mathrm{Cu}$ wire, with $10.5 \mathrm{~mm}$ in length and $5.5 \mathrm{~mm}$ in diameter. According to these dimensions, the calculated inductance of the coil is $2.73 \mu \mathrm{H}$. Figure 6.6 (a) shows a diagram of the coil geometry. The inductance and DC resistance of the coil were measured with a LCR meter, obtaining $L=2.8 \mu \mathrm{H}$, which results in an error of less than $3 \%$ in the estimated value, and $R=0.18 \Omega$. The coil is assembled onto a Quantum Design universal sample board (ref. P103C, shown in Fig. 6.6(b) and 6.6(c)) that plugs into the platform assembly of the MFP, which includes a calibrated cernox $^{\mathrm{TM}}$ (a zirconium oxynitride film) thermometer (see Fig. 6.6(d).). This sample board is easy to plug in and take out from the probe, so the coil does not need to be unsoldered and different coils can be used with the same circuit, if needed. A picture of the complete assembly at the bottom of the MFP is shown in Fig. 6.6(e). The coil dimensions are designed to hold a conventional gel-cap for PPMS and Magnetic Properties Measurement System (MPMS) measurements, so that it snugly fits into the coil core space.

The resulting oscillator waveform is shown in Fig. 6.7, measured with a Tektronix TDS7104 digital phosphor oscilloscope. The signal is nearly sinusoidal with an oscillation frequency around $9.2 \mathrm{MHz}$. In order to calculate the frequency and compare both experimental and calculated values, equation 


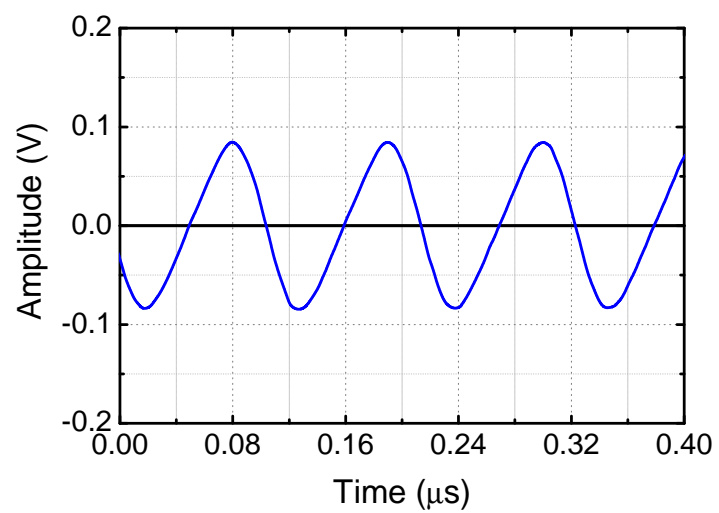

Figure 6.7: Measured output waveform at $\mathrm{V}_{\mathrm{DD}}=7 \mathrm{~V}, C=22 \mathrm{pF}$ and $L=2.8 \mu \mathrm{H}$.

6.20 may be approximated to $f \sim 1 /\left(2 \pi \sqrt{L C_{\text {total }}}\right)$ (since $\alpha \sim 0.4 \mathrm{mS}$ for the input voltage of $7 \mathrm{~V}$ in the measurement of Fig. 6.7, and $R=0.18 \Omega$, so that $R \alpha \ll 1)$. According to the values of $L=2.8 \mu \mathrm{H}$, the circuit capacitor, $C=22$ $\mathrm{pF}$, the capacitance and inductance contributions of the coax, $C_{\text {coax }} \sim 86 \mathrm{pF}$ and $L_{\text {coax }} \sim 200 \mathrm{mH}$, the calculated frequency is $9.26 \mathrm{MHz}$, giving a high accuracy between the calculated and experimental values, with an error of less than $1 \%$. Following the oscillation condition defined in equation 6.21 and the experimental results, the circuit oscillates with an input voltage, $\mathrm{V}_{\mathrm{DD}}$, ranging between $6.2 \mathrm{~V}$ and $13 \mathrm{~V}$, provided by a power supply EL302P programmable PSU.

The oscillation frequency of this designed rf self resonant system may be varied by correctly choosing those values of $L$ and $C$, so that equations 6.21 and 6.20 are fulfilled. In any case, the upper frequency is limited by the unavoidable parasitic capacitance of the coaxial cable $\left(C_{\text {coax }} \sim 86 \mathrm{pF}\right.$ for the one used in our design). For example, for $L=2.8 \mu \mathrm{H}$ used in this work, the highest frequency is around $10.2 \mathrm{MHz}$, and the lowest reachable frequency is around $5.4 \mathrm{MHz}$, the latter obtained with a $C=220 \mathrm{pF}$.

\subsection{Data acquisition}

The output signal of the circuit is read by an Agilent 53131A frequency counter and a Tektronix TDS2022B oscilloscope (see Fig. 6.2(a)). Data acquisition is performed by a connection via the IEEE - 488 General Purpose Interface Bus (GPIB) between the frequency counter and the Model 6000 PPMS respective port. Then, the resonating frequency acquired is used in the subsequent scripts written for measuring the sample TS. Measuring sequences have been imple- 
mented using the Visual Basic interpreter within MultiVu PPMS software. ${ }^{5}$ The executable programs scripted (.BAS files - called macros in MultiVu) allow scanning both $H_{\mathrm{DC}}$ field and $T$ (controlled by the PPMS) and measuring the resonance frequency, $f$. All measuring sequences are programmed directly on the PPMS controller computer and Multivu software, so that no additional equipment is needed for such effect. This fact represents an advantage with respect to similar TS measurement setups.

\subsection{Test Measurements}

\subsubsection{Test measurements on an empty coil}

First tests measurements were performed on the empty coil, so that we could study and characterize the response of the system resonance frequency to both $H_{\mathrm{DC}}$ and $T$. Such tests included those of stability of the frequency with time, temperature and field. Results for these tests are shown in Fig. 6.8.

The test of the resonance frequency with time for the CMOS circuit (Fig. $6.8(\mathrm{a}))$ reveals the existence of a drift, which is reduced with time from 30 $\mathrm{Hz} / \mathrm{min}$ to $14 \mathrm{~Hz} / \mathrm{min}$ in $150 \mathrm{~min}$ (see straight lines in Fig. 6.8(a)). This drift can be attributed to the time the electronics need to reach a thermal stability after they are turned on. Comparing this result with tests performed on a TDO based circuit, ${ }^{6}$ it is found that both circuits have analogous drifts, but the CMOS based circuit has a smoother and easy to correct curve on each TS scans. How these corrections are performed is described later in this section.

The variation of the frequency of the empty coil with magnetic field is shown in Fig. 6.8(b). The total shift in frequency for a complete $9 \mathrm{~T}$ unipolar scan (sweeping $H_{\mathrm{DC}}$ from $90 \mathrm{kOe}$ to $-90 \mathrm{kOe}$ ) is $197 \mathrm{~Hz}$.

Temperature dependence of the tank resonator frequency was probed by zero field cooling of the empty coil and it is shown in Fig. 6.8(c). This measurement supplies the background signal originated at the empty coil, $f_{\mathrm{bkg}}(T)$. Frequency monotonically increases as temperature is lowered. The total frequency drift with temperature was found to be around $133 \mathrm{~Hz} / \mathrm{K}$, showing that the system is very sensitive to thermal changes. This is mostly due to

\footnotetext{
${ }^{5}$ For further information see the PPMS Application Note 1070-209 of Quantum Design. www.qdusa.com/techsupport/index.html

${ }^{\circ} \mathrm{A}$ TDO based resonant circuit, similar to that described in Ref. 51. was also built by us to contrast the frequency stability results of both TDO and CMOS based resonant TS systems. The $f_{0}$ vs. time curve for the TDO configuration had an average drift of $5 \mathrm{~Hz} / \mathrm{min}$, after $120 \mathrm{~min}$. However, the frequency change with time in this case was not monotonic, showing a step-like curve that is not very simple to correct in subsequent TS scans.
} 

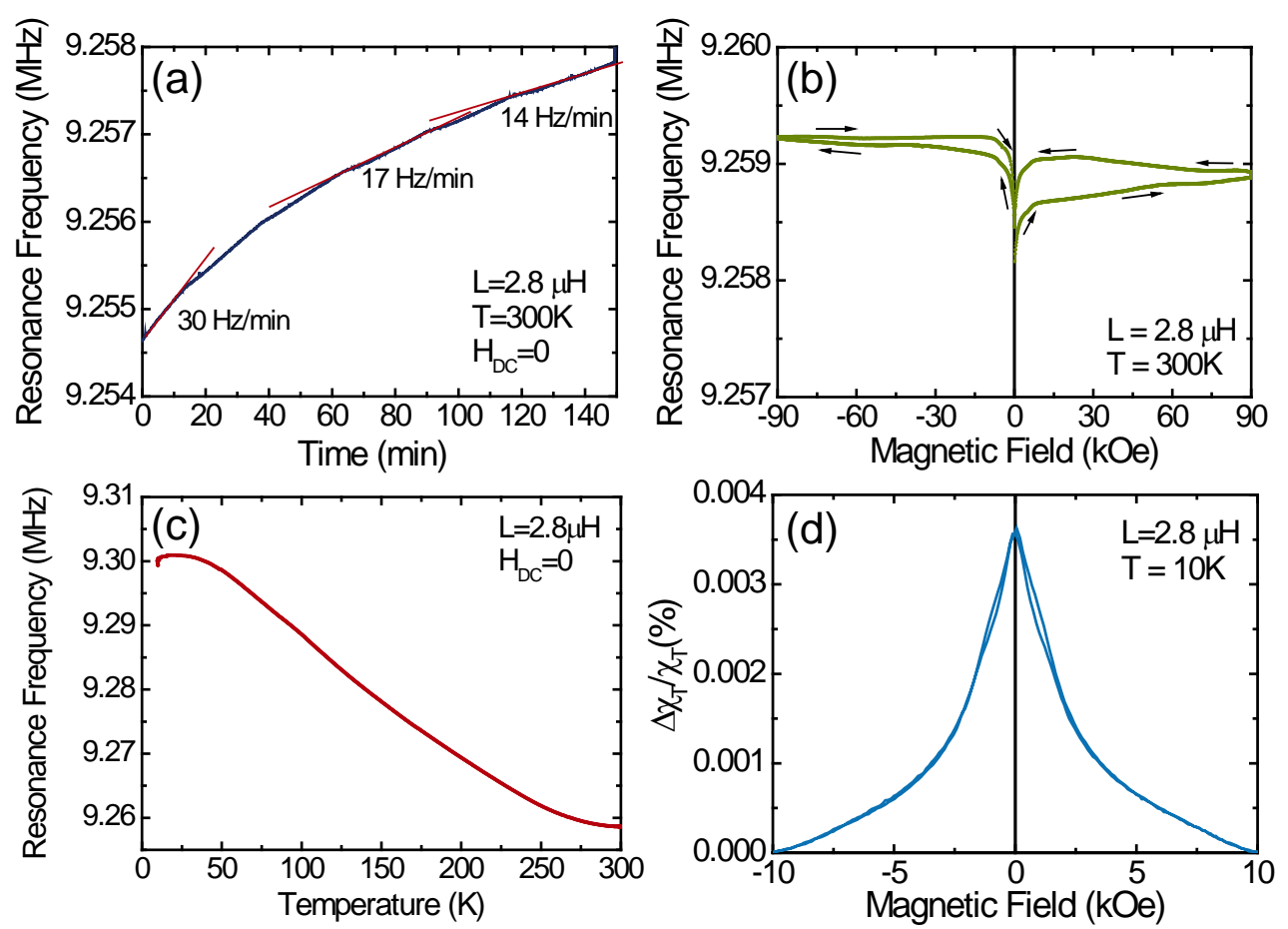

Figure 6.8: Test measurements of the frequency stability of the empty coil. (a) Drift with time at $300 \mathrm{~K}$. The straight segments indicate the rate of the frequency variation with time at different time intervals. (b) Variation with $H_{\mathrm{DC}}$ at $300 \mathrm{~K}$. Arrows show the direction of the field sweeping. (c) Variation with temperature at $H_{\mathrm{DC}}=0$. (d) TS scan at $T=10 \mathrm{~K}$ and with $H^{\text {sat }}=10 \mathrm{kOe}$, after corrections from drifts.

the thermal contraction the inductive coil suffers with decreasing temperature; the estimated rate of the frequency change as consequence of the changes in the dimension of the coil has been estimated as $\sim 100 \mathrm{~Hz} / \mathrm{K}$, calculated with the linear thermal expansion coefficient of copper $\left(\alpha=16.6 \times 10^{-6} \mathrm{~K}^{-1}\right)$.

A bipolar TS scan of the empty coil at $T=10 \mathrm{~K}$ was also performed (see Fig. $6.8(\mathrm{~d})$ ), where the change in resonance frequency was measured as $H_{\mathrm{DC}}$ was ramped from negative to positive saturation of $1 \mathrm{~T}$, and vice versa. We considered the quantity

$$
\frac{\Delta \chi_{\mathrm{T}}}{\chi_{\mathrm{T}}} \%=\frac{\chi_{\mathrm{T}}^{\mathrm{Sat}}-\chi_{\mathrm{T}}\left(H_{\mathrm{DC}}\right)}{\chi_{\mathrm{T}}^{\mathrm{Sat}}} \times 100 \propto \frac{\Delta f}{f_{0}} \%
$$

where $\chi_{T}^{\text {Sat }}$ is the transverse susceptibility at the saturating field $H^{\text {Sat }}=10$ kOe. This TS scan has been corrected for the previously described time and field linear drifts, by fitting a straight line between the data at positive and negative saturation and subtracting it from the whole data set. From Fig. 
$6.8(\mathrm{~d})$, the $\Delta \chi_{\mathrm{T}} / \chi_{\mathrm{T}} \%$ value at $H_{\mathrm{DC}}=0$ for the empty coil is $0.0036 \%$, which is very low compared to the $\Delta \chi_{\mathrm{T}} / \chi_{\mathrm{T}} \%$ we obtain for a magnetic sample, as we will demonstrate later.

In most cases this technique is applied to obtain intensive parameters, such as transition temperatures and fields, anisotropy, switching or coercivity fields, and, consequently, it is not necessary to determine the absolute $\chi_{\mathrm{T}}$. However, if desired, $\chi_{\mathrm{T}}$ may be scaled to absolute values by measuring the absolute ac longitudinal susceptibility, $\chi_{\mathrm{L}}$ in a SQUID magnetometer at $H_{\mathrm{DC}}=0$, and compare it with the results obtained in the transverse geometry for $H_{\mathrm{DC}}=0$.

\subsubsection{Calibration of the TS measurement system with $\mathrm{Gd}_{2} \mathrm{O}_{3}$}

The previously described procedure has been used to measure the $\chi_{\mathrm{T}}$ of a paramagnetic $\mathrm{Gd}_{2} \mathrm{O}_{3}$ sample, which is regularly used to calibrate different kinds of magnetometers, and allows us to probe and analyze the sensitivity of our equipment. The ac $\chi_{\mathrm{L}}$ was measured in a conventional SQUID magnetometer. Even though the frequency of the ac magnetic field in both measurements is different $\left(1 \mathrm{kHz}\right.$ for $\chi_{\mathrm{L}}$ and $9.2 \mathrm{MHz}$ for the $\left.\chi_{\mathrm{T}}\right)$, for this paramagnetic system the results should be identical since in both frequencies the susceptibility of the $\mathrm{Gd}_{2} \mathrm{O}_{3}$ is in its isothermal limit.

Figure 6.9 shows a plot of the relative change in the resonance frequency of the $L C$ tank in the TS setup with the sample $\Delta f / f_{0}$ vs. $\Delta \chi_{\mathrm{L}} / \chi_{0}$, at a common temperature range $(12-80 \mathrm{~K})$. The references $f_{0}$ and $\chi_{0}$ are taken as those values at $T=80 \mathrm{~K}$. A linear fit of the data yields to a slope of $s=0.607(1)$. This value is used to determine the scaling factor to transform frequency readings into absolute susceptibility units. Let us take the references $f_{0}$ and $\chi_{0}$ and define $a=s \frac{f_{0}}{\chi_{0}}$, so that $\Delta \chi_{\mathrm{L}}=a \Delta f$. We obtain $a=1.82 \times 10^{8}$ $\mathrm{emu} / \mathrm{Hz}$.

The sensitivity of the instrument can be evaluated according to the measurement signal to noise ratio (SNR)

$$
\mathrm{SNR}=\frac{\Delta f / f_{0}}{\delta\left(\Delta f / f_{0}\right)}
$$

SNR is on the order of $10^{3}$. Let us consider, as sensitivity test, a measurement of $\Delta f / f_{0}=10 \times \delta\left(\Delta f / f_{0}\right)$, so that we ensure that the signal measured is at least 10 times that of the noise. We obtain a sensitivity of $10 \times \delta\left(\Delta f / f_{0}\right) \times f_{0} / s_{1} \approx 2 \times 10^{-6} \mathrm{emu}$. 


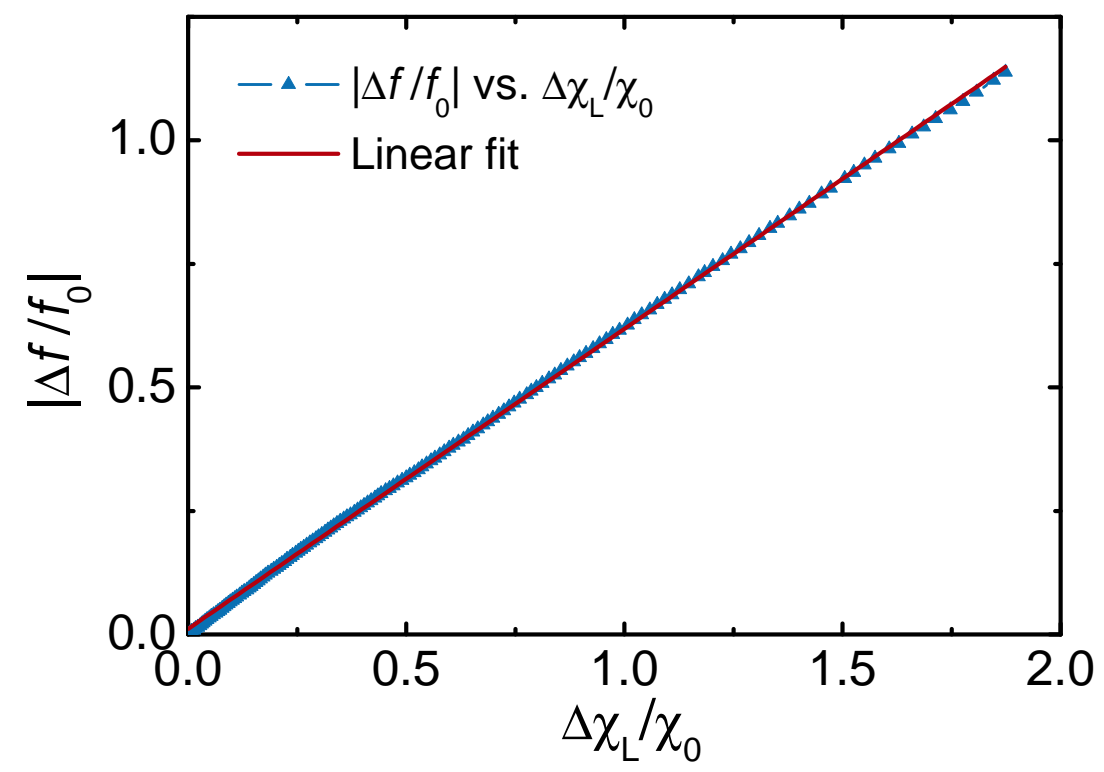

Figure 6.9: Measurements of the temperature dependence of the magnetic susceptibility of $\mathrm{Gd}_{2} \mathrm{O}_{3}$ : (solid line) $\Delta \chi_{\mathrm{L}} / \chi_{0}$ measured in a SQUID magnetometer, and (dashed line) relative change in the resonance frequency of the $L C$ tank in the TS setup with the sample. Inset: $\Delta f / f_{0}$ vs. $\Delta \chi_{\mathrm{L}} / \chi_{0}$, both at $H_{\mathrm{DC}}=0$ for the equivalent temperatures and fit of two linear regions in the data.

\subsubsection{Test measurements on a known and previously charac- terized sample with a TDO based TS system}

Additional tests of the equipment were performed by measuring a previously studied sample with a similar TDO-based TS system. For this, we have chosen a system of $\mathrm{Fe}_{3} \mathrm{O}_{4} \mathrm{NPs}$, with a mean diameter of $6 \mathrm{~nm}$, in powder form, synthesized and provided by Dr. Hariharan Srikanth and members of the Functional Materials Laboratory at the University of South Florida in Tampa, FL, USA. Magnetic properties of this and similar NPs systems can be found in Ref. [48]. Tests on this sample included bipolar TS scans at fixed temperatures with $H^{\text {Sat }}=1 \mathrm{~T}$ performed with the two different TS setups: the TDO based and our improved CMOS-based circuit. Fig. 6.10(a) shows a 3D plot of scans measured at different temperatures between $1.8 \mathrm{~K}$ and $100 \mathrm{~K}$ with the CMOSbased system. A detailed bipolar scan for the sample measured at $1.8 \mathrm{~K}$ is shown in Fig. 6.10(b). The respective and previously described corrections for the time and field linear drifts have been performed on the scans. Two peaks are identified for each unipolar scan, which correspond to the anisotropy fields, $H_{\mathrm{K}}$, according to the theoretical model of TS developed by Aharoni et al. (see section 6.1). A clear evolution of the $H_{\mathrm{K}}$ peaks in the sample with temperature is observed in the $3 \mathrm{D}$ plot (Fig. 6.10(a)); i.e., the double peak 
structure becomes a single peak one as the temperature increases. Such an evolution with temperature is better illustrated in the $H_{\mathrm{K}}$ vs. $T$ plot in Fig. 6.10 (c). It is observed that the described merging of the double peak structure occurs at the blocking temperature $(\sim 70 \mathrm{~K})$.

The direct comparison of the results obtained with our CMOS-based TS setup with respect to those with a TDO-based circuit are shown in Fig. 6.11, where we have plotted bipolar scans for the sample measured at $10 \mathrm{~K}$ with both setups. Our CMOS-based system reproduces the shape and position of the peaks at $H_{\mathrm{K}}$ of the TS scan measured with a TDO-based circuit setup built by us, and also of those previously reported in Ref. [48」 performed with a similar TDO-based TS equipment. Moreover, the TS amplitude in our CMOS-based TS setup is enhanced by a factor of almost two at $10 \mathrm{~K}$ for the same sample, which demonstrates the high sensitivity of our equipment. The latter is also accentuated by the comparison with the practically flat TS signal for the empty coil, shown in dashes in the same Fig. 6.11 for comparison (notice that this correspond to the same curve shown in Fig. 6.8(d)), which is negligible compared to that of the sample.
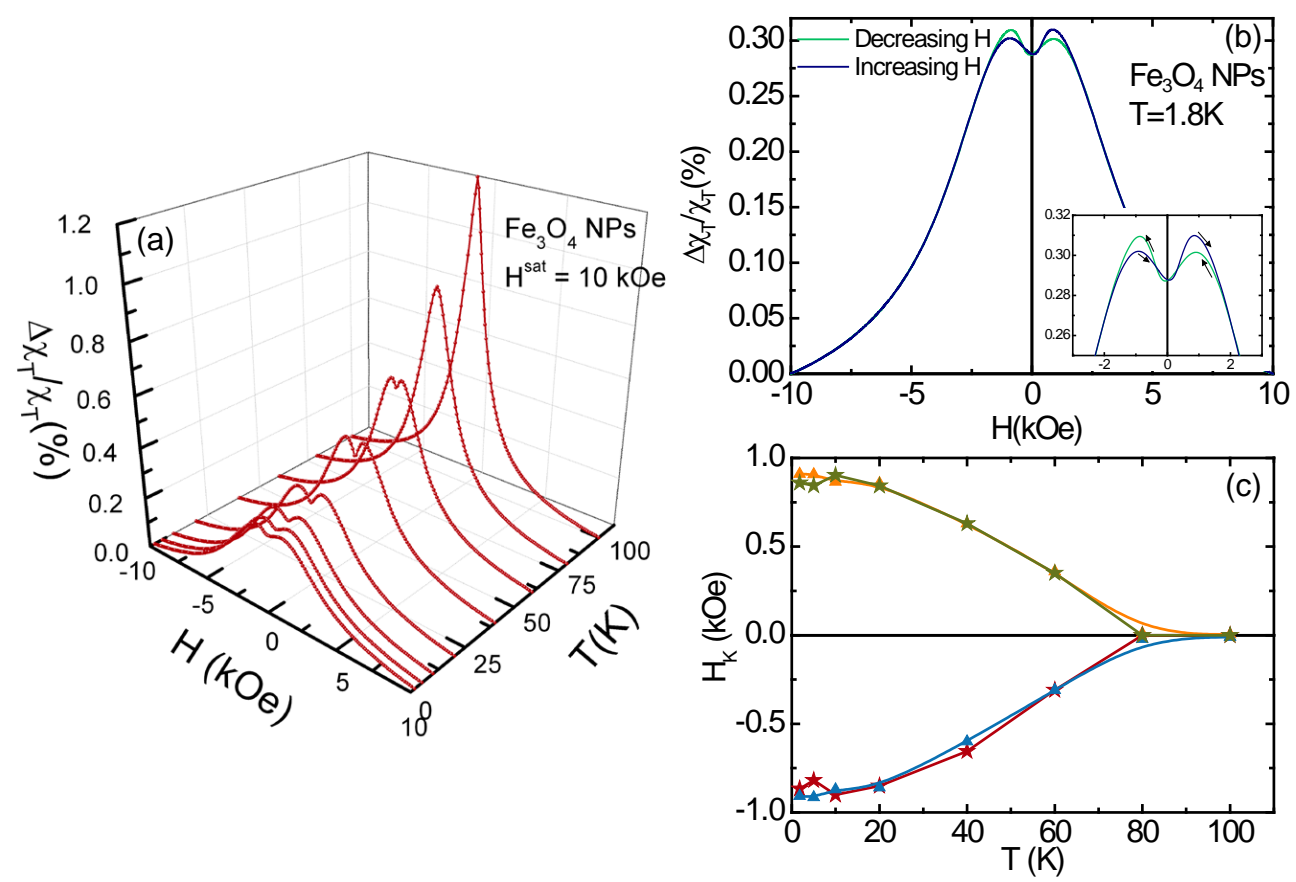

Figure 6.10: TS test measurements on $\mathrm{a} \mathrm{Fe}_{3} \mathrm{O}_{4}$ nanoparticles samples. (a) 3D plot of unipolar scans. (b) Bipolar scan at $1.8 \mathrm{~K}$. Inset: detail of the anisotropy peaks of this scan. (c) Temperature dependence of $H_{\mathrm{K}}$. 


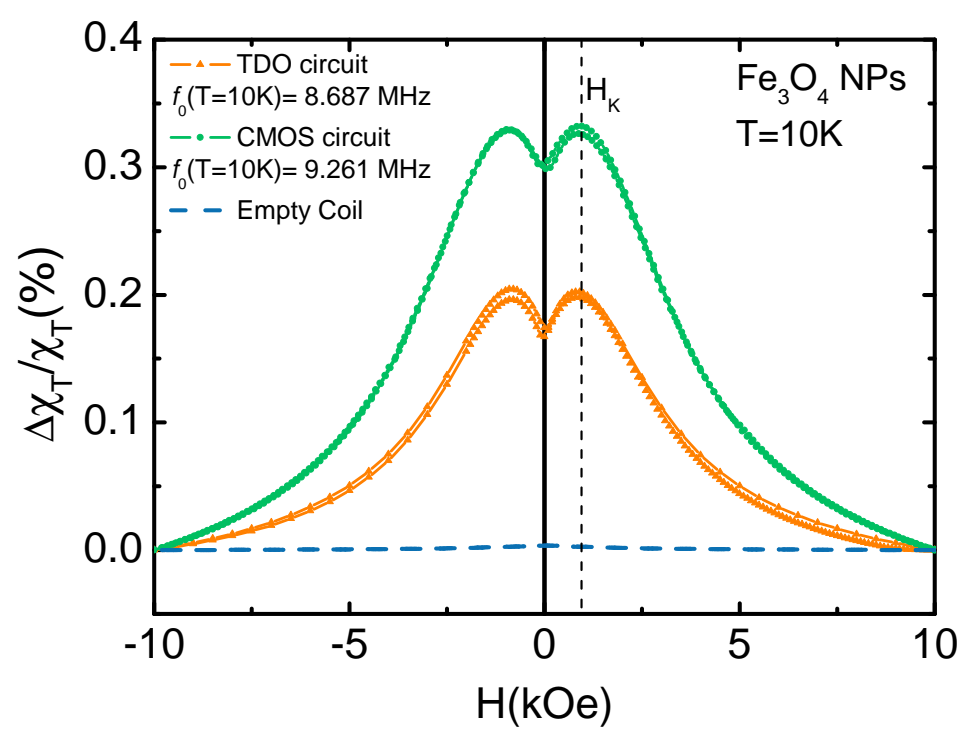

Figure 6.11: Comparison of bipolar TS scans on a $\mathrm{Fe}_{3} \mathrm{O}_{4}$ NPs sample at $10 \mathrm{~K}$ with $H^{\text {Sat }}=10 \mathrm{kOe}$ performed with a TDO based $(\Delta)$, and with a CMOS based $(\bullet)$ TS setup. The measurement on the empty coil (--) is also shown for comparison.

\subsubsection{Low temperature measurements on $\mathrm{TmCo}_{2}$}

Finally, in order to probe the functionality of the TS system at low temperatures, we have performed measurements on a sample of bulk $\mathrm{TmCo}_{2}$ : a Laves phase with a ferrimagnetic to paramagnetic transition temperature around 4 K [180]. Previous works have demonstrated that this TS technique is well suited to track down and probe interesting magnetic features in similar $\mathrm{RCo}_{2}$ Laves phases $[37,38]$. For the particular case of $\mathrm{TmCo}_{2}$ we use it to track its magnetic transition at low temperatures (below $10 \mathrm{~K}$ ). TS measurements on this sample have been performed with a saturating field of $10 \mathrm{kOe}$ from $2 \mathrm{~K}$ to $20 \mathrm{~K}$.

Figure 6.12(a) shows selected TS bipolar scans measured for the $\mathrm{TmCo}_{2}$ sample. Each TS profile in the ferrimagnetic regime (below $T \sim 4 \mathrm{~K}$ ) shows pronounced peaks at characteristic fields, which we identified as switching fields, $H_{\mathrm{S}}$. In Fig. 6.12(b) we have plotted the values of such $H_{\mathrm{S}}$ as a function of temperature. Close to $T_{\mathrm{c}} \sim 4 \mathrm{~K}$ the peaks are narrower and their values suffer a slight increase (indicated by arrows in Fig. 6.12(b)), similar to what has been previously observed for similar $\mathrm{RCo}_{2}$ systems $\left[37,38\right.$. Above $T_{\mathrm{c}}$, $H_{\mathrm{S}}$ values rapidly decrease since the sample enters to a magnetic disordered state, but surprisingly they never reach a zero value in this temperature range. The existence of finite $H_{\mathrm{S}}$ in the paramagnetic state of these Laves phases has been ascribed to the occurrence of magnetic clusters, a novel and fascinating 

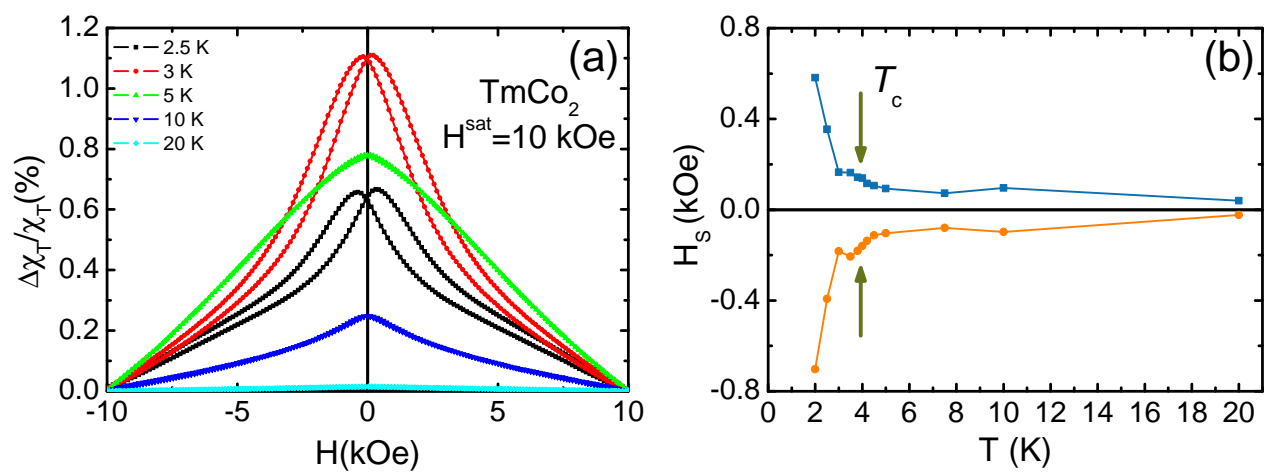

Figure 6.12: (a) TS bipolar scans of $\mathrm{TmCo}_{2}$ for selected temperatures between 2 20 K. (b) Switching field values, $H_{S}$, identified from the TS profiles.

phenomenon coined as parimagnetism $[37,38,181\rfloor$.

\subsection{Conclusions}

A complete TS measurement equipment for the PPMS has been implemented, operating at the $\mathrm{MHz}$ frequency range, with a wide temperature and $H_{\mathrm{DC}}$ field range of operation. The efficiency, simplicity and versatility of the proposed CMOS oscillator based on a $L C$ cross-coupled topology guarantee reliability, robustness and portability. Measurement tests demonstrate frequency stability and high performance of the circuit, as well as high sensitivity to probe the transverse susceptibility of interesting magnetic systems. Also, we have demonstrated that our system is well suited to study magnetic phenomena at low temperatures, down to $T=1.8 \mathrm{~K}$. 



\section{Chapter 7}

\section{Transverse susceptibility of iron oxide nanoparticles systems}

In this section we introduce two examples of iron oxide nanoparticles systems studied via $\mathrm{rf}$ transverse susceptibility (TS). We demonstrate possibilities of the TS technique, extensively described in the previous chapter, to directly probe the magnetic anisotropy of magnetic systems.

In the first example, we present dc SQUID based magnetometry and radio frequency transverse susceptibility measurements on oleic acid- and $\mathrm{SiO}_{2}$ coated magnetite $\left(\mathrm{Fe}_{3-\mathrm{x}} \mathrm{O}_{4}\right)$ nanoparticles. The effects of the type of coating on the magnetic anisotropy are evaluated as a function of the particle size ( 5 $\mathrm{nm}$ and $14 \mathrm{~nm}$ of average diameter). Coating the samples with $\mathrm{SiO}_{2}$ reduces the interparticle interactions compared to those oleic acid coated samples, but it seems to be more effective for the particles with $5 \mathrm{~nm}$ than for those with $14 \mathrm{~nm}$ in diameter. $\mathrm{SiO}_{2}$ coating also decreases the magnetic anisotropy field measured on the particles, with respect to the oleic acid-coated samples. This is relevant concerning applications in biomedicine, since the $\mathrm{SiO}_{2}$ coating renders 5 and $14 \mathrm{~nm}$ hydrophilic particles with very limited agglomeration, low anisotropy, and superparamagnetic behavior at room temperature.

The second example consists in maghemite $\left(\gamma-\mathrm{Fe}_{2} \mathrm{O}_{3}\right)$ nanoparticles of different sizes, on which the effective anisotropy constant, $K_{\text {eff }}$ is estimated from the TS measurements and its variation is examined as a function of the particle size. The resulting values range from 4 to $8 \times 10^{4} \mathrm{erg} / \mathrm{cm}^{3}$, being on the order of the magnetocrystalline anisotropy in bulk maghemite. $K_{\text {eff }}$ values increase as the particle diameter increases. Evidences of anisotropy field 
distribution given by the size distribution in the samples, and interparticle interactions that increase as the particle size increases, are also observed in the TS measurements.

\subsection{Magnetite Nanoparticles}

Magnetite $\left(\mathrm{Fe}_{3} \mathrm{O}_{4}\right)$ NPs are one of the most commonly studied nanoparticles systems because of their relatively easy production by chemical routes, low toxicity and fascinating magnetic properties $\lfloor 182-184\rfloor$. It has been demonstrated that the surrounding environment of the $\mathrm{Fe}_{3} \mathrm{O}_{4}$ particles strongly affects their magnetic properties $\lfloor 185-189\rfloor$, in such a way that, for example, bulk-like structural, magnetic and electronic behavior may be recovered in $5 \mathrm{~nm}$ particles [182-184]. Such effects are obtained in $\mathrm{Fe}_{3} \mathrm{O}_{4}$ NPs with oleic acid used as surfactant, covalently bonded to the particle surface. Those particles are good candidates for biomedical applications [190], once the hydrophobic oleic acidcoating is ligand-exchanged to a hydrophilic one, which makes the particles dispersible in aqueous media. Within this framework, magnetic NPs of a few nanometers in size and with the highest possible magnetization are required. They must also show superparamagnetic behavior at room temperature and low interparticle interactions, for which low particle agglomeration is desirable. All these features enable the synthesis of NP dispersions (ferrofluids) which can be injected into biological systems for in vivo applications $\lfloor 3,5\rfloor$.

Following this scheme, it is crucial to evaluate the effects of the hydrophilic particle coating on the magnetic anisotropy of $\mathrm{Fe}_{3} \mathrm{O}_{4} \mathrm{NPs}$, in order to get optimum particles for bio-applications. Particle coating also reduces the interparticle interactions, thus shifting the superparamagnetic transition well below room temperature. This allows the use of larger particles with larger magnetic moment, which results in larger signals in the superparamagnetic regime $\lfloor 191\rfloor$.

Then, we use a hydrophilic coating, namely, $\mathrm{SiO}_{2}$ on $5 \mathrm{~nm}$ and $14 \mathrm{~nm} \mathrm{Fe}_{3} \mathrm{O}_{4}$ NPs, and examine its effects on the magnetic anisotropy of the particles by RF transverse susceptibility measurements. For completeness, we compare the results on these $\mathrm{SiO}_{2}$-coated $\mathrm{Fe}_{3} \mathrm{O}_{4}$ NPs with those obtained for the starting oleic acid-coated particles of the same size.

\subsubsection{Experimental}

The synthesis of the size controlled oleic acid-coated NPs was carried out by the group of Dr. Amilcar Labarta and Dr. Xavier Batlle from the Depar- 
tament de Física Fonamental and Institut de Nanociencia i Nanotecnologia, Universitat de Barcelona, in Barcelona, Spain. It was performed following conventional thermal decomposition of an iron precursor in an organic medium in the presence of an organic acid as a surfactant, as extensively described elsewhere $\lfloor 187,192-194\rfloor$. Samples with $5 \mathrm{~nm}$ and $14 \mathrm{~nm}$ particles were prepared. For $\mathrm{SiO}_{2}$ coating, the hydrolysis of tetra ethoxy silane (TEOS) in water-inoil (w/o) micro-emulsions has proven to be a reproducible method for the subsequent silica coating of the hydrophobic oleic acid-coated magnetite NPs $\lfloor 195,196\rfloor$. The proportion of TEOS and the concentration of magnetite cores of known diameter in the medium allows controlling the shell thickness as well as the number of magnetic cores inside a single shell.

The size and shape of the NPs were examined by transmission electron microscopy (TEM) performed by the group of Dr. Amilcar Labarta and Dr. Xavier Batlle from the Departament de Física Fonamental and Institut de Nanociencia i Nanotecnologia, Universitat de Barcelona, in Barcelona, Spain. TEM measurements were carried out on Hitachi H-7500 (low-resolution) and JEOL JEM 2010 (high-resolution) transmission electron microscopes of the Centres Científics i Tecnològics of the Universitat de Barcelona. Samples for the TEM study were prepared on copper grids coated with carbon. A drop of NP's solution was carefully placed on the copper grid surface and dried. The size distributions of the nanocrystals were determined by analysis of the TEM images.

The bright field (BF) TEM image of each sample is shown in Fig. 7.1 along with the size distributions obtained for each case. The oleic acid-coated particles are very uniform (Fig. 7.1(a) and 7.1(b)), similarly to those previously reported for this range of sizes. $\mathrm{SiO}_{2}$-coated particles are shown in Fig. 7.1(c) and 7.1(d). Darker and lighter regions correspond to the $\mathrm{Fe}_{3-\mathrm{x}} \mathrm{O}_{4}$ core (5 and $14 \mathrm{~nm}$ average diameter) and the $\mathrm{SiO}_{2}$ shell (about 15-20 nm thick in both cases), respectively, as shown in detail in the inset of Fig. 7.1(d). These images reveal that the $\mathrm{Fe}_{3-\mathrm{x}} \mathrm{O}_{4}$ NPs are well single-coated, with absence of agglomeration, though an excess of $\mathrm{SiO}_{2}$ is observed from the presence of several $\mathrm{SiO}_{2}$ beads without $\mathrm{Fe}_{3-\mathrm{x}} \mathrm{O}_{4}$ cores. The average diameter and width obtained from the size distributions for the four samples are listed in table 7.1.

\subsubsection{Magnetic Properties}

The magnetic properties of the particles have been determined by dc magnetic susceptibility, $\chi_{\mathrm{dc}}(T)$, and RF transverse susceptibility, performed in the four $\mathrm{Fe}_{3-\mathrm{x}} \mathrm{O}_{4}$ NPs samples. $\chi_{\mathrm{dc}}(T)$ was measured in a SQUID magnetometer at the Servicio de Medidas Físicas of the Universidad de Zaragoza, after zero-field 

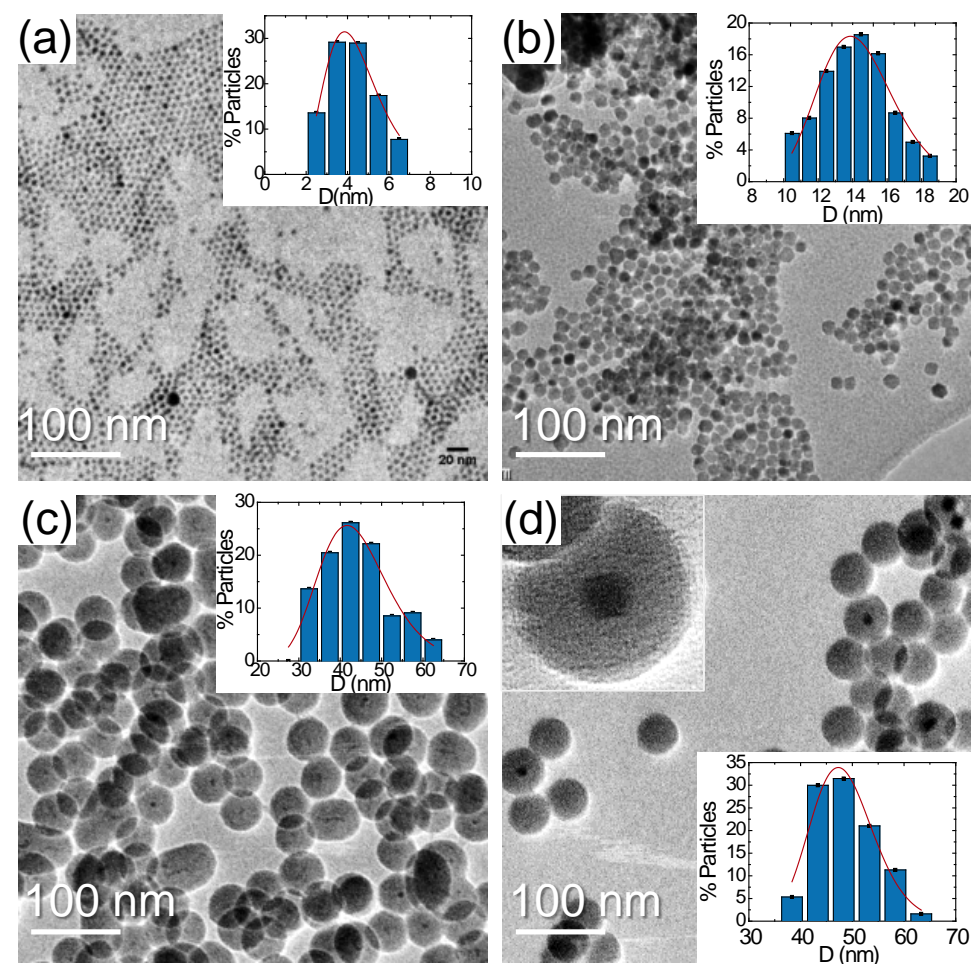

Figure 7.1: BF TEM images of the oleic acid-coated ((a) and (b)) and $\mathrm{SiO}_{2}$-coated ((c) and (d)) $\mathrm{Fe}_{3-\mathrm{x}} \mathrm{O}_{4}$ NPs. Insets shows the size distributions obtained in each case. Inset in (d) shows details of a $\mathrm{SiO}_{2}$ coated particle. The $\mathrm{SiO}_{2}$ shell thickness in (c) and (d) is about $15-20 \mathrm{~nm}$.

cooling (ZFC) and field cooling (FC) the sample. Temperature was varied between 2 and $300 \mathrm{~K}$, and a 50 Oe field was applied.

The ZFC-FC curves shown in Fig. 7.2 reveal the superparamagnetic behavior of the $\mathrm{Fe}_{3-\mathrm{x}} \mathrm{O}_{4}$ particles. At lower temperatures, $\mathrm{FC}$ and $\mathrm{ZFC}$ for each sample separate, and the ZFC curve shows a maximum at a blocking temperature, $T_{B}$. The blocking temperature of the particles with average sizes of 5 and $14 \mathrm{~nm}$, is 35 and $300 \mathrm{~K}$, respectively, while the $\mathrm{SiO}_{2}$-coated samples exhibit a reduced $T_{B}$ (see table 7.1 ) as compared to the oleic acid-coated samples.

TS measurements were performed using the setup described in chapter 6 . The temperature, $T$, and static magnetic field, $H_{\mathrm{dc}}$, are varied using the PPMS within $2-300 \mathrm{~K}$ and $\pm 15 \mathrm{kOe}$, respectively. Figure $7.3(\mathrm{a})$ shows the $3 \mathrm{D}$ plot of unipolar TS scans for the $5 \mathrm{~nm}$ oleic acid $\mathrm{Fe}_{3-\mathrm{x}} \mathrm{O}_{4}$ NPs. At low temperatures the curves show two peaks located symmetrically about the origin of the magnetic field axis, characteristic of the blocked regime. According to the theoretical model of TS developed by Aharoni et al. «177」. (see section 6.1), a unipolar scan should reveal the existence of those two symmetric peaks at the 


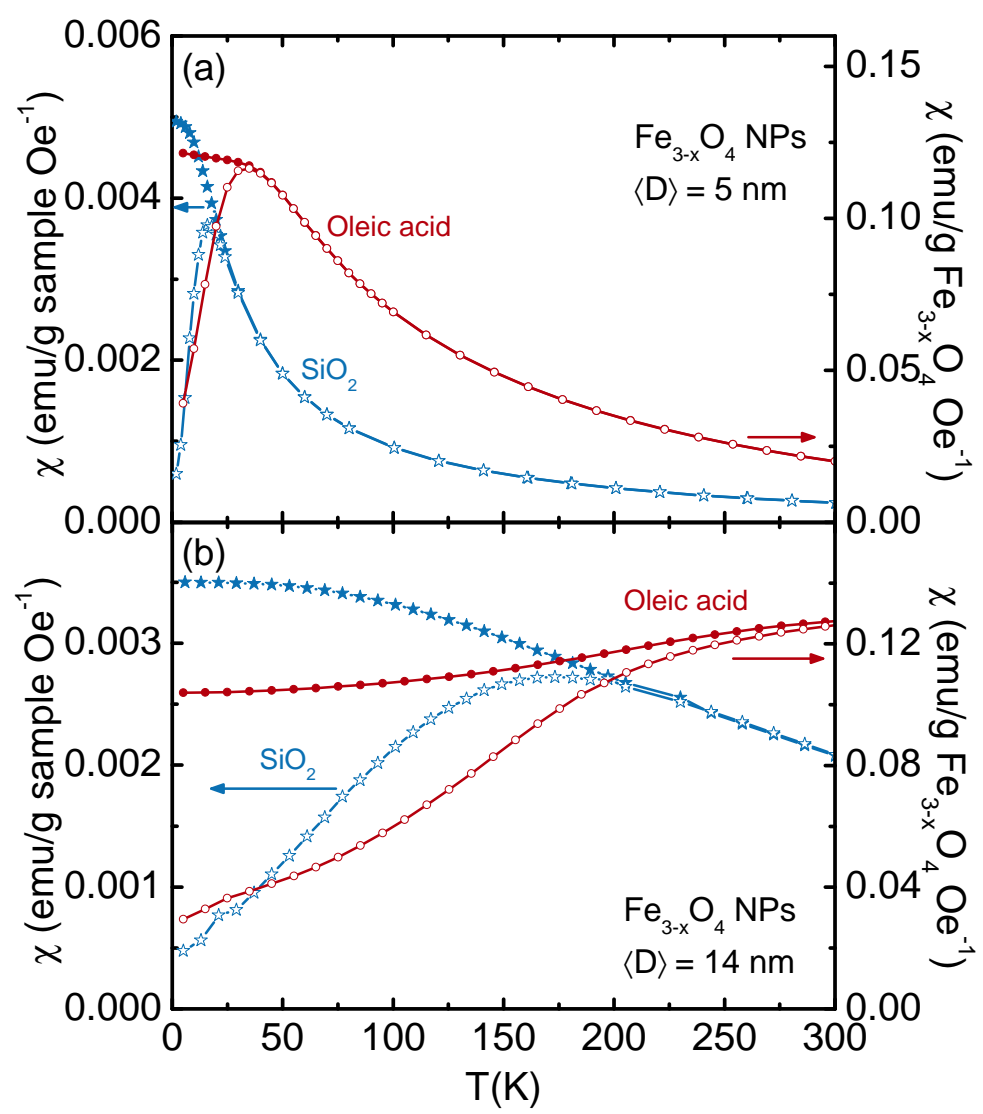

Figure 7.2: ZFC-FC curves measured for the oleic acid- and $\mathrm{SiO}_{2}$-coated $\mathrm{Fe}_{3-\mathrm{x}} \mathrm{O}_{4}$ NPs with average diameters of (a) $5 \mathrm{~nm}$ and (b) 10-12 nm.

anisotropy fields, $H_{\mathrm{K}}$, and a third one at the switching field, $H_{\mathrm{S}}$. The absence of the peak at $H_{\mathrm{S}}$ in the TS scans, as well as the rounded characteristic shape of the peaks observed in our case or in similar NP systems [47], are attributed to the presence of a distribution of anisotropy fields, which causes the peak at the switching field to merge indistinguishably with one of the peaks at the anisotropy field [45]. The distribution of anisotropy fields is an unavoidable consequence of the dispersion in particle size.

As temperature increases from $2 \mathrm{~K}$, the double-peak structure of the isothermal unipolar scans becomes less pronounced and merges into a single central peak, as depicted in Fig. 7.3(b). This trend is consistent with a gradual transition from a blocked state towards a superparamagnetic one, which will be discussed further in section 7.1.3. Analogous results for the TS measurements have been obtained for the other three samples.

The $H_{\mathrm{K}}$ values obtained from the TS profiles for the four samples are plotted in Fig. 7.4, as a function of temperature. Let us remark that the 
Table 7.1: Summary of parameters of the $\mathrm{Fe}_{3-\mathrm{x}} \mathrm{O}_{4}$ NPs. Average particle diameter $(\langle D\rangle)$ and distribution width $(\sigma)$ as obtained from TEM, blocking temperature $\left(T_{B}\right)$ obtained from $\chi_{\mathrm{dc}}$, blocking temperature $\left(T_{M}\right)$ deduced from TS, and the intrinsic anisotropy field obtained at $T=2 \mathrm{~K}\left(H_{\mathrm{K} 0}\right)$.

\begin{tabular}{cccccc}
\hline \hline Coating & $\begin{array}{c}\langle D\rangle \\
(\mathrm{nm})\end{array}$ & $\begin{array}{c}\sigma \\
(\mathrm{nm})\end{array}$ & $\begin{array}{c}T_{B} \text { from } \\
\chi_{\mathrm{dc}}(\mathrm{K})\end{array}$ & $\begin{array}{c}T_{M} \text { from } \\
\mathrm{TS}(\mathrm{K})\end{array}$ & $\begin{array}{c}H_{\mathrm{K} 0} \\
(\mathrm{kOe})\end{array}$ \\
\hline oleic & 5 & 3 & $35(2)$ & $35(2)$ & $0.69(3)$ \\
acid & 14 & 9 & $\sim 300$ & $215(5)$ & $0.90(4)$ \\
\hline \multirow{2}{*}{$\mathrm{SiO}_{2}$} & 44 & 27 & $16(1)$ & $14(1)$ & $0.65(3)$ \\
& 49 & 29 & $170(1)$ & $140(1)$ & $0.89(4)$ \\
\hline \hline
\end{tabular}
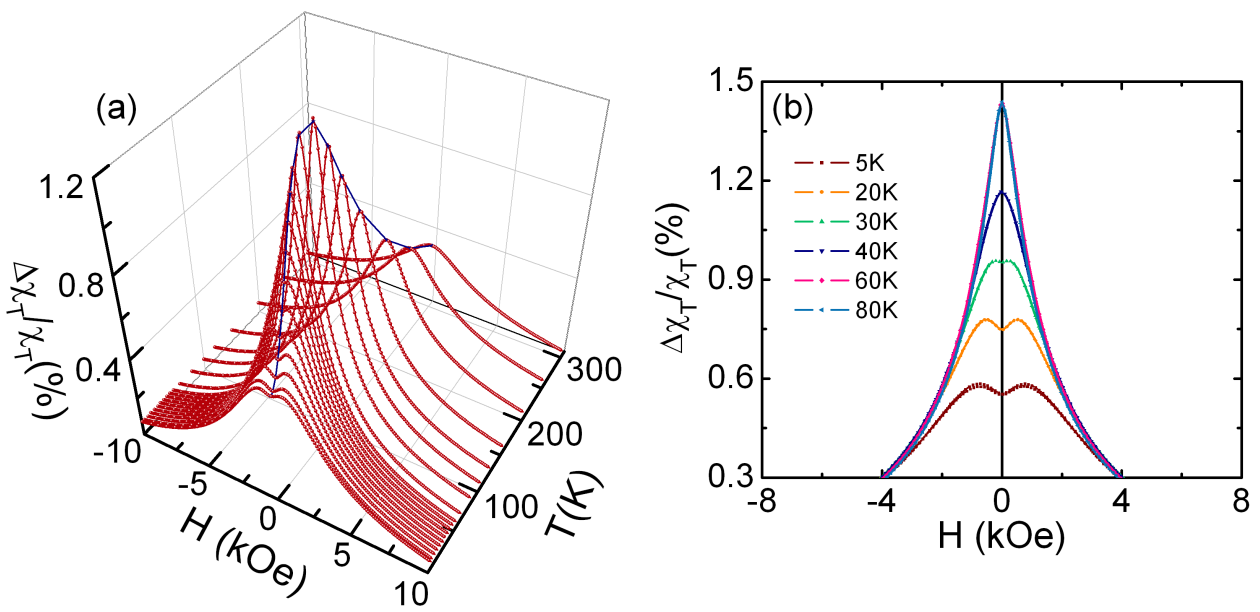

Figure 7.3: TS results for $5 \mathrm{~nm}$ oleic acid-coated $\mathrm{Fe}_{3-\mathrm{x}} \mathrm{O}_{4}$ NPs. (a) 3D plot of TS unipolar scans at several temperatures. (b) TS bipolar scans at selected temperatures.

direct determination of $H_{\mathrm{K}}$ is one of the main assets of the TS technique. We define $T_{M}$ as the minimum temperature at which $H_{\mathrm{K}}=0$, corresponding to the blocking temperature where the double peak structure in the TS profiles disappears. $T_{M}$ values obtained for the four samples are listed in table 7.1. Note that these values are well in agreement with those of $T_{B}$ obtained from the ZFC curves measured by conventional SQUID magnetometry (also listed in table 7.1).

$H_{\mathrm{K}}$ values are found to be lower than $1 \mathrm{kOe}$ in all cases. For most temperatures, $H_{\mathrm{K}}$ are higher for the oleic acid- than for the $\mathrm{SiO}_{2}$-coated samples (see Fig. 7.4), for both particle sizes. This is due to a reduction in the $T_{B}$ value for the $\mathrm{SiO}_{2}$-coated particles, fact that will be also discussed in section 7.1.3. At about $2 \mathrm{~K}$, though, $H_{\mathrm{K}}$ values are very close for each group of particles $(5 \mathrm{~nm}$ with both coatings on one side, and $14 \mathrm{~nm}$ with both coatings on the other), 


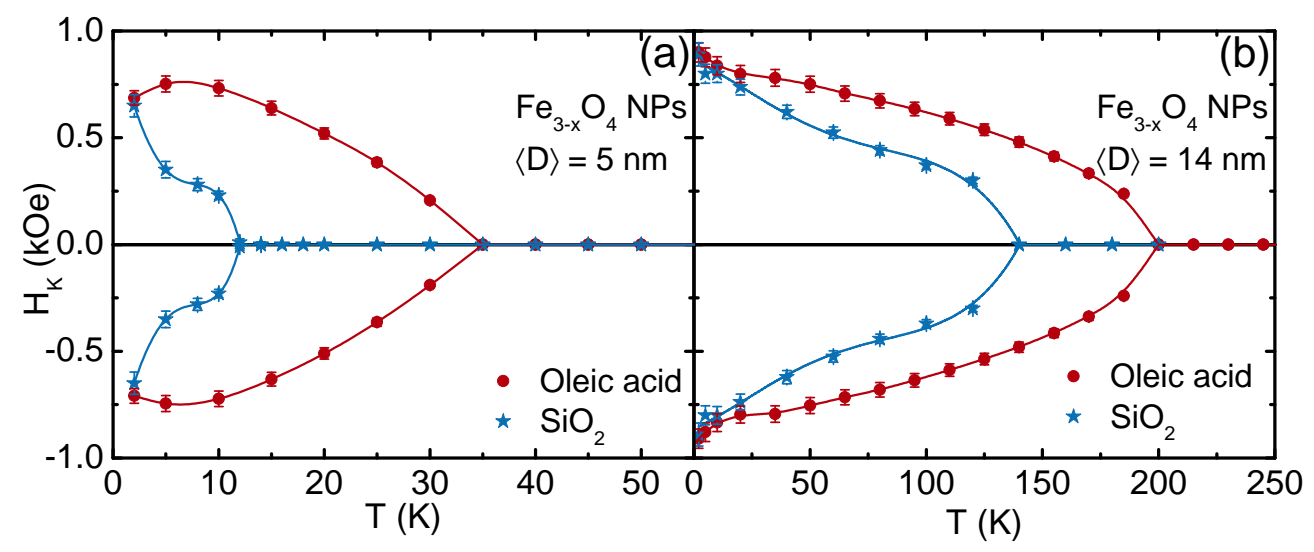

Figure 7.4: Comparison of the temperature dependence of the values of the anisotropy field $\left(H_{\mathrm{K}}\right)$ extracted from the TS scans for the oleic acid- and $\mathrm{SiO}_{2}$-coated $\mathrm{Fe}_{3-\mathrm{x}} \mathrm{O}_{4}$ NPs with average diameters of (a) $5 \mathrm{~nm}$ and (b) $14 \mathrm{~nm}$.

which reveals that at very low temperature the strong magnetic behavior of the magnetite core overcomes any effect of the coating.

\subsubsection{Discussion}

In order to provide a deeper understanding of the TS measurements, we perform a direct comparison between the ZFC curve as obtained from the $\chi_{\mathrm{dc}}$ SQUID measurements $\lfloor 185,187\rfloor$ (top panel,left-hand side y-axis) and the TS ratio, $\Delta \chi_{\mathrm{T}} / \chi_{\mathrm{T}}(T)$, at $H_{\mathrm{dc}}=0$ (top panel, right-hand side y-axis), for the $5 \mathrm{~nm}$ oleic-coated particles, which is shown in Fig. 7.5 as a function of temperature. As the temperature increases, the TS ratio at $H_{\mathrm{dc}}=0$ increases, similarly to the observed behavior of the ZFC curve, although it has a maximum at a higher temperature (see Fig. 7.5). This is consistent with the well-known increase $[1]$ in the temperature of the ac susceptibility maximum as the exciting frequency increases (with respect to the dc measurements), since TS is measured at a frequency of $12 \mathrm{MHz}$. Such a feature for TS has been previously described in reference $\left\lfloor 176\right.$. Finally, both $\chi_{\mathrm{dc}}$ and TS measurements coincide in the paramagnetic region, as it has been proven in section 6.5.2.

For completeness, the temperature dependence of the positive $H_{\mathrm{K}}$ values is plotted on the same graph (bottom panel, left-hand side y-axis). Notice the nice accordance between $T_{M}$ from TS and $T_{B}$ from the maximum of the ZFC curve (see dashed line in Fig. 7.5), as mentioned in the previous section, demonstrating that in the blocked state (below $T_{B}$ ) the particles have finite $H_{\mathrm{K}}$ values.

The strong temperature dependence of $H_{\mathrm{K}}$, observed in Figs. 7.4 and 7.5, 


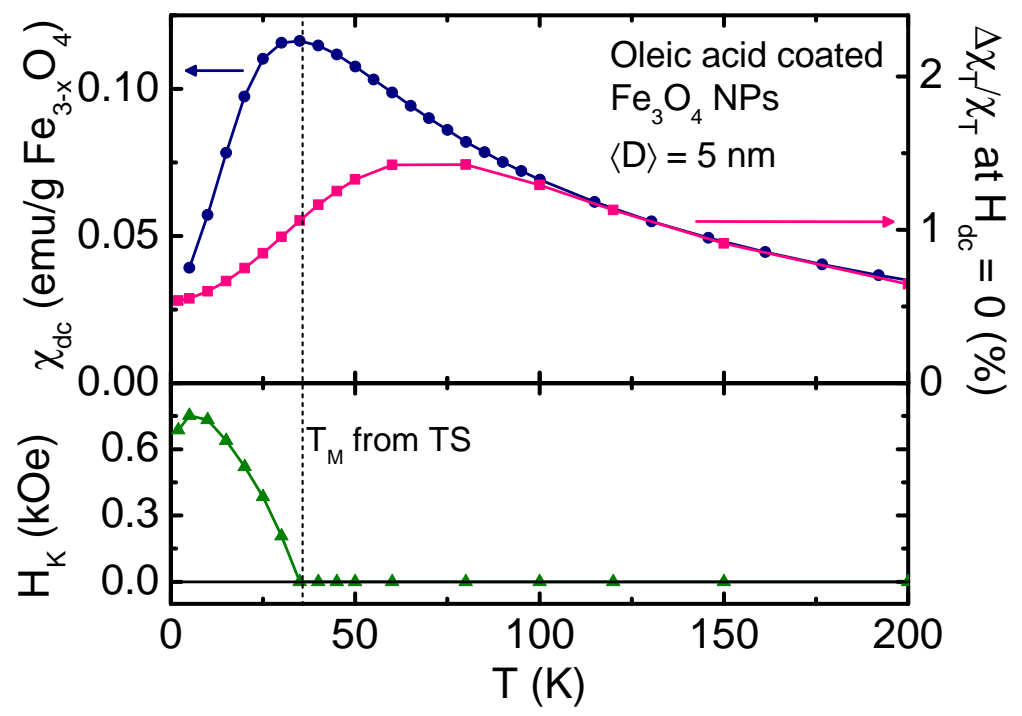

Figure 7.5: Comparison of the temperature dependence of (i) the ZFC curve $(\bullet)$ obtained from dc susceptibility measurements $\left(\chi_{\mathrm{dc}}\right)$ at 50 Oe; (ii) the curve obtained from the transverse susceptibility values at $H_{\mathrm{dc}}=0(\square)$, and (iii) the anisotropy field $\left(H_{\mathrm{K}}\right)$, for the oleic acid-coated $5 \mathrm{~nm} \mathrm{Fe}_{3-\mathrm{x}} \mathrm{O}_{4}$ particles. Dashed line marks the $T_{B}$ from the ZFC curve, which matches the blocking temperature, $T_{M}$, obtained from TS.

accounts for both the thermal variation of the magnetocrystalline anisotropy constant of the material, which is negligible in this case, and for the influence of the thermal energy that overcomes the anisotropy energy of the particles $[197,198]$. The decrease of $H_{\mathrm{K}}$ with increasing $T$ is usually described by the relation $H_{\mathrm{K}}=H_{\mathrm{K} 0}\left[1-\left(T / T_{M}\right)^{\beta}\right]$, where $\beta \sim 0.5\lfloor 199,200$, for an assembly of aligned particles, and $\beta \sim 0.77$ for an assembly of randomly oriented particles [201-203], and $H_{\mathrm{K} 0}$ corresponds to the intrinsic anisotropy field of the material. The emerging picture is that, at low temperatures, the magnetite particles are frozen and $H_{\mathrm{K}} \sim H_{\mathrm{K} 0}$. As $T$ increases, some fraction of the particles become superparamagnetic since the thermal excitations are now capable of reversing the magnetic moment from one orientation to the opposite, and the effective $H_{\mathrm{K}}$ decreases. Thus, well above the blocking temperature, all the particles are superparamagnetic, which is characterized by the random thermal fluctuations of single-domain particle moments, so that the measured $H_{\mathrm{K}}$ of the collection of magnetite particles is zero. However, the curves of $H_{\mathrm{K}}(T)$ in Fig. 7.4 and 7.5 do not follow the simple $H_{\mathrm{K}}=H_{\mathrm{K} 0}\left[1-\left(T / T_{M}\right)^{\beta}\right]$ relation, since additional effects on the magnetic anisotropy of the particles, such as interparticle interactions [204] and anisotropy field distribution [199, 200 ], should be taken into account.

$T_{M}$ values, and, correspondingly, $T_{B}$, are found to decrease as the particle 
diameter decreases, from $215 \mathrm{~K}$ to $35 \mathrm{~K}$ for $14 \mathrm{~nm}$ and $5 \mathrm{~nm}$ oleic acid-coated $\mathrm{NPs}$, respectively, and from $140 \mathrm{~K}$ to $14 \mathrm{~K}$ for the equivalent $\mathrm{SiO}_{2}$-coated particles (see table 7.1 and Fig. 7.4). This coating also reduces the values of $T_{M}$, with respect to those found for the oleic acid-coated case with the same diameter, which is related to the reduction of the dipolar interparticle interactions [47 as consequence of the increased magnetite interparticle corecore distance by the thick $\mathrm{SiO}_{2}$ shell.

A stronger reduction in $T_{M}$ by $\mathrm{SiO}_{2}$ coating is observed for the smaller 5 nm particles than for the larger $14 \mathrm{~nm}$ NPs, despite the $\mathrm{SiO}_{2}$ shell thickness is roughly the same in both cases, since their magnetic moment is reduced with the particle volume, and, correspondingly, their dipole-dipole interactions decrease. Consequently, the smaller the particles the more effective the reduction of the interparticle interactions by $\mathrm{SiO}_{2}$ coating.

According to these analyzes, with the TS measurements we are observing, on the one hand, a phenomenon related to a dc behavior of the particles, i.e., their superparamagnetic-blocking transition occurring at a characteristic temperature, detectable from the $H_{\mathrm{K}}$ vs. $T$ plot and the ZFC from $\chi_{\mathrm{dc}}$ measurements; and, on the other hand, a dynamic process evidenced in the shift of the ac susceptibility maximum to higher temperatures with increasing frequency. This is possible given that in a TS experiment we are applying both a bias and a dynamic magnetic field at the same time, thus we are able to observe and disentangle both phenomena in the particles.

Figure 7.6 shows the comparison of the ZFC curves and the TS ratio at $H_{\mathrm{dc}}=0$ as a function of temperature for the samples with $14 \mathrm{~nm}$ in diameter with both coatings. Since these bigger particles have a wider distribution in size and their interparticle interactions are more evident than the case of the smaller particles in Fig. 7.5, the peak at the ZFC curve is broader. However, $T_{M}$ obtained from TS seems to coincide with the inflection point of the ZFC curve. At any rate, the value of the blocking temperature is sharper from the TS study ( $H_{\mathrm{K}}$ vs. $T$ plot) than from these ZFC measurements, the latter showing a broad maximum.

Finally, an estimation of the effective anisotropy constant, $K_{\text {eff }}$ may be directly deduced from the $M_{\mathrm{S}}$ measured from conventional SQUID magnetometry and the values of $H_{\mathrm{K} 0}$ obtained from the TS measurements at low temperatures (see table 7.1), as $K_{\mathrm{eff}}=1 / 2 \quad M_{\mathrm{S}} H_{\mathrm{K} 0}\lfloor 191\rfloor$. With $M_{\mathrm{S}}$ for all samples of the sizes studied here being on the order of $80-90 \mathrm{emu} / \mathrm{g} \mathrm{Fe}_{3-\mathrm{x}} \mathrm{O}_{4}$ $\lfloor 185,187\rfloor$, the resulting $K_{\text {eff }}$ values are within $1.6-1.8 \times 10^{5} \mathrm{erg} / \mathrm{cm}^{3}$, which are close to those values reported for both bulk magnetite $\left(\sim 1-3 \times 10^{5}\right.$ $\left.\mathrm{erg} / \mathrm{cm}^{3}\right)\lfloor 191,205,206\rfloor$, and high quality $5 \mathrm{~nm}$ particles $\left(\sim 4-5 \times 10^{5}\right.$ $\left.\mathrm{erg} / \mathrm{cm}^{3}\right)\lfloor 185,206\rfloor$. 


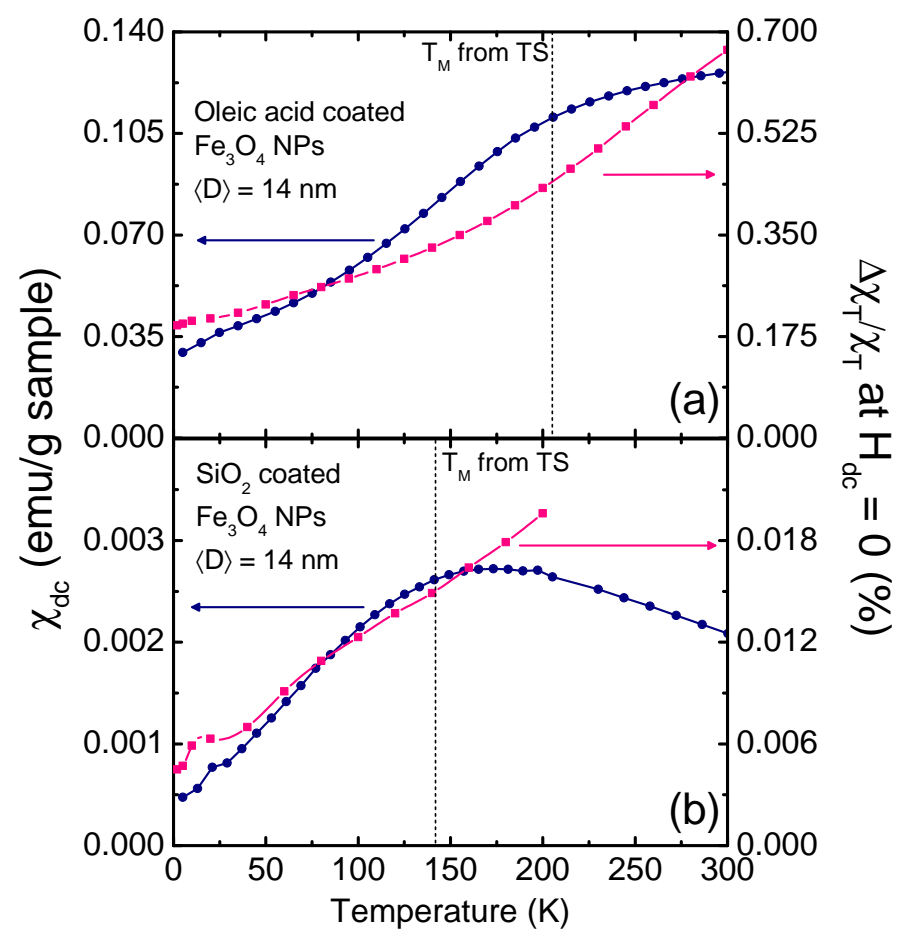

Figure 7.6: Comparison of the ZFC curve from $\chi_{\mathrm{dc}}(\bullet)$ with the TS at $H_{\mathrm{dc}}=0(\square)$, both as a function of $T$, for the $\mathrm{Fe}_{3-\mathrm{x}} \mathrm{O}_{4}$ NPs with 10-12 nm in diameter: (a) oleic acid coated and (b) $\mathrm{SiO}_{2}$ coated. Dashed line marks $T_{M}$.

\subsubsection{Conclusions}

We have demonstrated that $\mathrm{SiO}_{2}$ coating of the $\mathrm{Fe}_{3-\mathrm{x}} \mathrm{O}_{4}$ particles reduces the interparticle interactions, which is revealed by the reduction of the blocking temperature of the system. This is observed for both particle sizes but it is more efficient in the case of the smaller ones. $H_{\mathrm{K}}$ values of the oleic acid-coated NPs are higher than those of the $\mathrm{SiO}_{2}$-coated ones because of their higher $T_{B}$, and, consequently, larger interparticle interactions. This suggests that the effective anisotropy field increases with interparticle dipole-dipole interactions, which is consistent to what has been described in references $\lfloor 1\rfloor$ and [185」. TS measurements also allow estimating the effective magnetic anisotropy constant, obtaining values in close agreement with others published in literature.

To sum up, let us recall that biomedical applications require hydrophilic particles with high saturation magnetization to manipulate and detect them by application of an external magnetic field, low anisotropy field and superparamagnetic behavior at room temperature so that particle agglomeration, as due to attractive magnetic forces, is avoided $[3,5\rfloor$. The present work indicates that our 5 and $14 \mathrm{~nm} \mathrm{SiO}_{2}$-coated hydrophilic magnetite nanoparticles 
fulfill all those requirements (saturation magnetization close to that of the bulk magnetite, $H_{\mathrm{K}}$ below $1 \mathrm{kOe}$ and superparamagnetic behavior at room temperature), which makes them very attractive for such purposes.

\subsection{Maghemite Nanoparticles}

The chemical stability of the maghemite phase $\left(\gamma-\mathrm{Fe}_{2} \mathrm{O}_{3}\right)$ of iron oxide nanoparticles makes this system very attractive for technological and biomedical applications; this feature is specially important for the latter, since, beside the requirements described in the previous section, particles with low degradability into biological systems for in vivo applications are desired $[3,5\rfloor$. However, and despite all the research carried out on maghemite NPs, their magnetic behavior is not yet well understood.

Bulk maghemite has a inverse-spinel structure with some vacant sites, exhibiting ferrimagnetic ordering below $918 \mathrm{~K}$. Maghemite nanoparticles show reduced saturation magnetization compared to the bulk, they do not saturate at rather high fields, they show open and shifted hysteresis loops, and irreversibility in ZFC-FC curves $[207$. This magnetic behavior has been attributed to finite size and surface effects at the maghemite particles [208]. Accordingly, some theoretical models have been developed to explain such a behavior, in particular the decrease in their magnetization with size reduction; from these models, the most accepted is the one based on a bulk-like ferrimagnetic core and a shell composed of disordered moments [207]. The magnetism of the $\gamma-\mathrm{Fe}_{2} \mathrm{O}_{3}$ particles we study here may be understood in the frame of such a core-shell model, which we summarize as follows $[207,209$ : the particles consist of a core with structural periodicity exhibiting a superparamagnetic behavior, and a disordered shell without the periodicity. Most of the magnetic contribution of the entire particle usually originates from the bulk-like ferrimagnetic core. The disordered shell at the surface is analyzed as paramagnetic or antiferromagnetic component in the magnetization data at high fields. The magnetic interaction between the core and the shell can appear as an exchange bias effect. The shell has a thickness of about $1 \mathrm{~nm}$, independently of the size of the particle $[207,209\rfloor$.

A previous study on the effects of pressure on these maghemite nanoparticles allowed to disentangle the contribution of the core $\left(K_{\text {core }}\right)$ and the surface $\left(K_{\mathrm{S}}\right)$ to the effective magnetic anisotropy of the system, $K_{\text {eff }}\lfloor 210\rfloor$. The values of $K_{\text {core }}$, on the one hand, were found to be between that from the magnetocrystalline anisotropy for bulk maghemite and that usually found in maghemite nanoparticles $\left(10^{5}-10^{6} \mathrm{erg} / \mathrm{cm}^{3}\right)$. On the other hand, $K_{\mathrm{S}}$ is similar to that previously found for maghemite nanoparticles $\left(10^{-2} \mathrm{erg} / \mathrm{cm}^{2}\right)$. In 
this section, we study the magnetic anisotropy of maghemite nanoparticles of different sizes, estimated from the direct measurement of the anisotropy field $H_{\mathrm{K}}$ from $\mathrm{rf}$ transverse susceptibility measurements.

The series of maghemite nanoparticles samples studied here were synthesized and previously characterized by the group of Dr. Angel Millán and Dr. Fernando Palacio of the Instituto de Ciencia de Materiales de Aragón, CSICUniversidad de Zaragoza. The particles are dispersed in a polymer matrix with low size dispersion and regular distribution of particles. A total of four $\gamma-\mathrm{Fe}_{2} \mathrm{O}_{3}$ NPs samples were studied, with average diameters of 5.2, 6, 7 and $13 \mathrm{~nm}$, labeled S5 to S8 following the nomenclature used in Refs. [207, 209.. The NPs diameter has been determined by transmission electron microscopy (TEM) and small angle X-ray scattering (SAXS), as described in the same references $[207,209\rfloor$, measurements carried out by the same group of Dr. Millán and Dr. Palacio. The average diameter and width obtained from the size distributions for the four samples are listed in table 7.2.

\subsubsection{TS measurements}

We have performed TS measurements on the four samples of maghemite nanoparticles using the setup described in chapter 6 . The temperature, $T$, and static magnetic field, $H_{\mathrm{dc}}$, are varied using the PPMS within 2-300 K and $\pm 10 \mathrm{kOe}$, respectively. In Fig. 7.7(a) we show the 3D plot of unipolar TS scans at several temperatures for particles with $\langle D\rangle=5.2(9) \mathrm{nm}$. The TS profiles are similar to those described in section 7.1.2 for magnetite NPs. The peak at the switching field merges with one of the anisotropy field peaks due to the presence of a distribution in anisotropy fields in the maghemite NPs system, as described above for the $\mathrm{Fe}_{3-x} \mathrm{O}_{4}$ NPs. However, in the case of $\gamma-\mathrm{Fe}_{2} \mathrm{O}_{3}$ NPs, the two peaks observed at the anisotropy field have different heights. As

Table 7.2: Summary of the parameters deduced from the TEM and magnetic measurements on $\gamma-\mathrm{Fe}_{2} \mathrm{O}_{3}$ NPs. Average particle diameter $(\langle D\rangle)$ and width $(\sigma)$ of the size distribution obtained from TEM, blocking temperature $\left(T_{M}\right)$ obtained from TS, saturation magnetization $\left(M_{\mathrm{S}}\right)$, anisotropy field $\left(H_{\mathrm{K}}\right)$ and anisotropy constant $\left(K_{\mathrm{eff}}\right)$, these last three parameters at $T=5 \mathrm{~K}$.

\begin{tabular}{ccccccc}
\hline \hline Sample & $\begin{array}{c}\langle D\rangle \\
(\mathrm{nm})\end{array}$ & $\begin{array}{c}\sigma \\
(\mathrm{nm})\end{array}$ & $\begin{array}{c}T_{M} \\
(\mathrm{~K})\end{array}$ & $\begin{array}{c}M_{\mathrm{S}} \\
\left(\mathrm{emu} / \mathrm{cm}^{3}\right)\end{array}$ & $\begin{array}{c}H_{\mathrm{K}} \\
(\mathrm{Oe})\end{array}$ & $\begin{array}{c}K_{\text {eff }} \\
\left(\mathrm{erg} / \mathrm{cm}^{3}\right)\end{array}$ \\
\hline S5 & $5.2(9)$ & 0.9 & $25(5)$ & $217(9)$ & $340(30)$ & $3.7(5) \times 10^{4}$ \\
S6 & $6(1)$ & 1.1 & $105(5)$ & $237(9)$ & $460(30)$ & $5.4(8) \times 10^{4}$ \\
S7 & $7(1)$ & 1.4 & $140(8)$ & $210(9)$ & $570(40)$ & $6.0(8) \times 10^{4}$ \\
S8 & $13(3)$ & 3.2 & $250(10)$ & $356(9)$ & $440(30)$ & $7.8(9) \times 10^{4}$ \\
\hline \hline
\end{tabular}




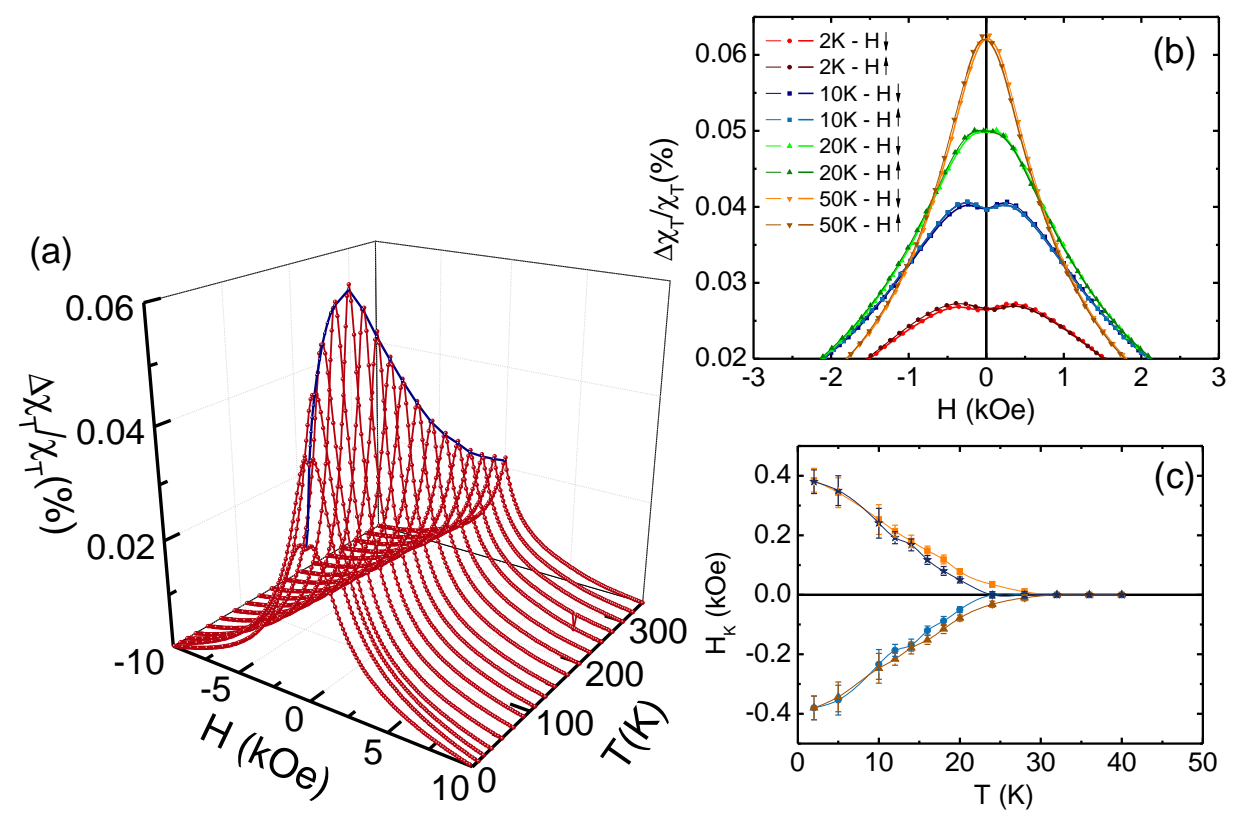

Figure 7.7: TS results the sample with $\langle D\rangle=5.2(9) \mathrm{nm}$. (a) 3D plot of TS unipolar scans at several temperatures. (b) TS bipolar scans at selected temperatures. (c) Values of $H_{\mathrm{K}}$ extracted from the TS scans plotted as a function of $T$.

temperature increases from $2 \mathrm{~K}$, the peak heights difference is reduced, and the double-peak structure is less pronounced, evolving into a single central peak, as depicted in the TS bipolar scans plotted for selected temperatures in Fig. 7.7(b). This trend, similar to the case of $\mathrm{Fe}_{3-x} \mathrm{O}_{4} \mathrm{NPs}$, is consistent with the gradual transition from a blocked state towards a superparamagnetic one. A better illustration of this evolution is observed in the plot of the $H_{\mathrm{K}}$ values (obtained directly from the TS scans) as a function of $T$ shown in Fig. 7.7(c). From these plots, the $T_{M}$ of each sample is directly obtained as the minimum temperature where $H_{\mathrm{K}}=0$.

Analogous results for the TS measurements have been obtained for the other three samples. $T_{M}$ values for the four samples are listed in table 7.2. They are found to decrease as the NP diameter decreases, from $250 \mathrm{~K}$ down to $25 \mathrm{~K}$ for particles of $13 \mathrm{~nm}$ and $5.2 \mathrm{~nm}$, respectively. This trend in $T_{M}$ may be understood as due to the decrease of the particles magnetic moment with their reduced volume, and, correspondingly, the decrease of their dipole-dipole interactions, similarly to the case of $\mathrm{Fe}_{3-x} \mathrm{O}_{4}$ NPs. 


\subsubsection{Discussion}

As it has been mentioned above, there is a difference in the peaks height of the TS profiles, so that for each unipolar scan from positive to negative field sweep, the peak at the first quadrant is always larger than the peak height in the second one. This feature is better illustrated in Fig. 7.8 where details of a TS bipolar scan of sample S5 (Fig. 7.8(a)) and S8 (Fig. 7.8(a)) measured at $T=5 \mathrm{~K}$ are shown. One way to analyze such an asymmetry is to quantify the peak difference for each unipolar TS scan as

$$
\Delta \text { height }(\%)=\frac{\left[(\text { Peak height })_{\text {first quadrant }}-(\text { Peak height })_{\text {second quadrant }}\right]}{(\text { Peak height })_{\text {first quadrant }}} \%
$$

similar to the procedure described in Ref. [47]. In Fig. 7.10(a) we have plotted $\Delta$ height as a function of the reduced temperature $T / T_{M}$ for the four samples in the series. It is evident that the asymmetry is found to increase as the particle size increases. This is a fact that has been consistently observed in several TS studies of NPs systems and has been related to the presence of anisotropy field dispersion $\lfloor 45\rfloor$, and also as a consequence of increasing interparticle interactions with the particle size [47]. Both components might be present in our maghemite NPs samples since, on one hand, previous studies of their size have shown a broader size distribution for the sample with larger diameter than those with smaller ones $\lfloor 207,209$, and, on the other hand, effects of interparticle interactions have been observed in ac susceptibility measurements at different frequencies in these samples (measurements performed by the group of Dr. Millán and Dr. Palacio). The extrapolated values of the microscopic characteristic time, $\tau_{0}$, are obtained from a typical Arrhenius plot of $\ln (1 / \omega)$ vs. $1 / T_{B}$ shown in Fig. 7.9 , from frequency dependent ac susceptibility measurements. For non-interacting superparamagnetic maghemite nanoparticles $\tau_{0}$ values are usually on the order of $10^{-10} \mathrm{~s}$, while in the present case, $\tau_{0}$ obtained from linear fits of data shown in Fig. 7.9 reaches $10^{-15} \mathrm{~s}$ for the particles with $\mathrm{D}=13 \mathrm{~nm}$. Such a strong reduction in $\tau_{0}$ is a signature of systems where interparticle interactions are important [211].

The thermal evolution of $H_{\mathrm{K}}$ for the four samples is shown in Fig. 7.10(b). $H_{\mathrm{K}}$ are also plotted as a function of the reduced temperature $T / T_{M}$ in order to have better comparison. $H_{\mathrm{K}}$ values at low temperatures are of the same order for all samples, except for those particles of smallest size. As temperature increases, $H_{\mathrm{K}}$ values seem to follow a general trend of increasing as the the particle diameter increases, which is reflected in the effective anisotropy values as it is described below.

The effective anisotropy constant, $K_{\text {eff }}$ may be estimated from the $M_{\mathrm{S}}$ ob- 


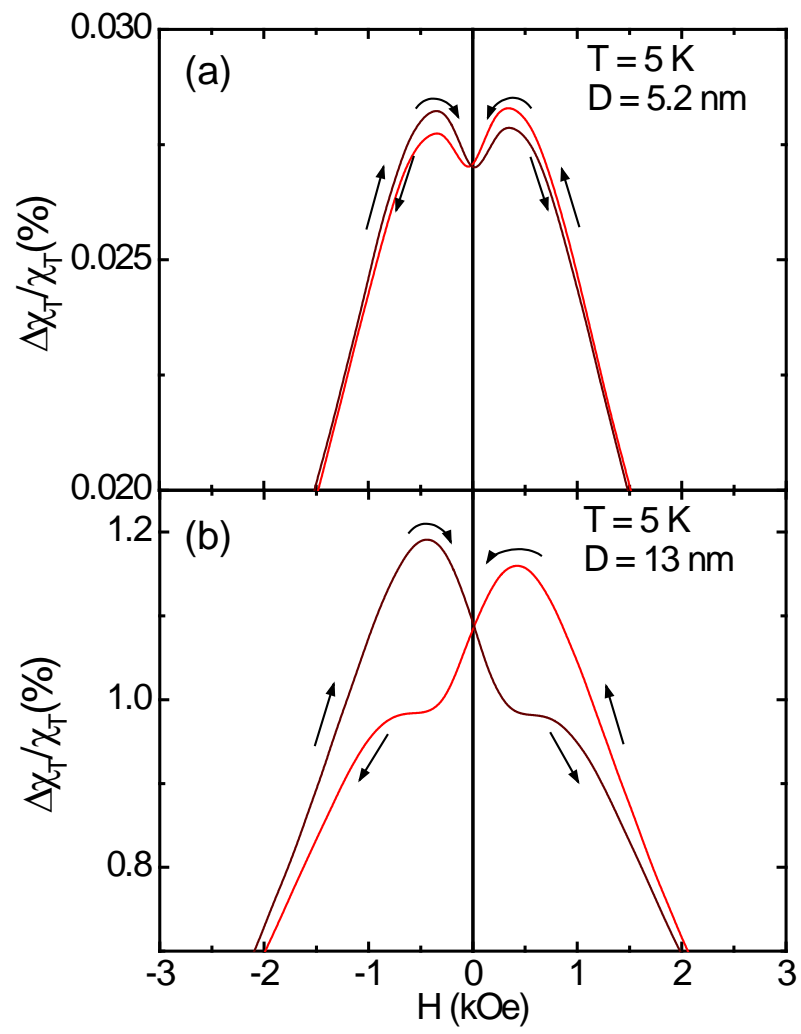

Figure 7.8: Comparison of the details of the TS bipolar scans measured at $T=5$ $\mathrm{K}$ for the sample with (a) $\langle D\rangle=5.2 \mathrm{~nm}$ (S5) and that with (b) $\langle D\rangle=13 \mathrm{~nm}$ (S8). Arrows show the direction of the field sweep.

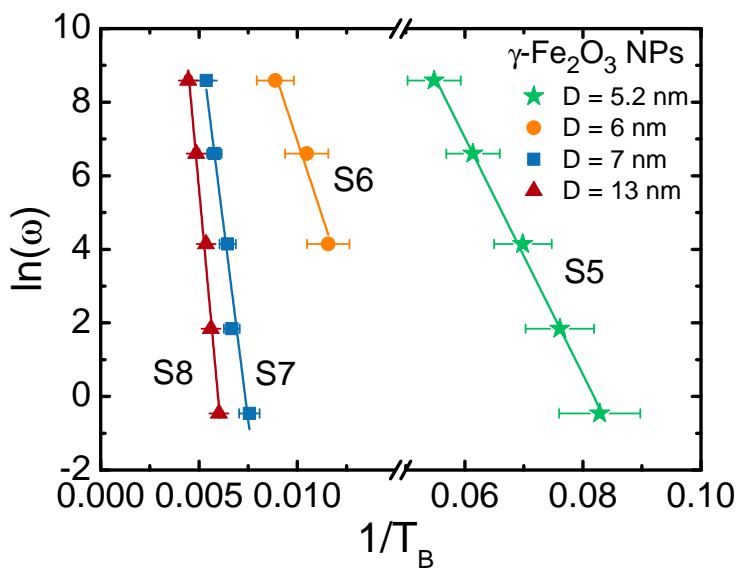

Figure 7.9: Arrhenius plot of the measuring time $(1 / \omega)$ as a function of $1 / T_{B}$, as obtained from ac susceptibility measurements in the four maghemite NPs. 


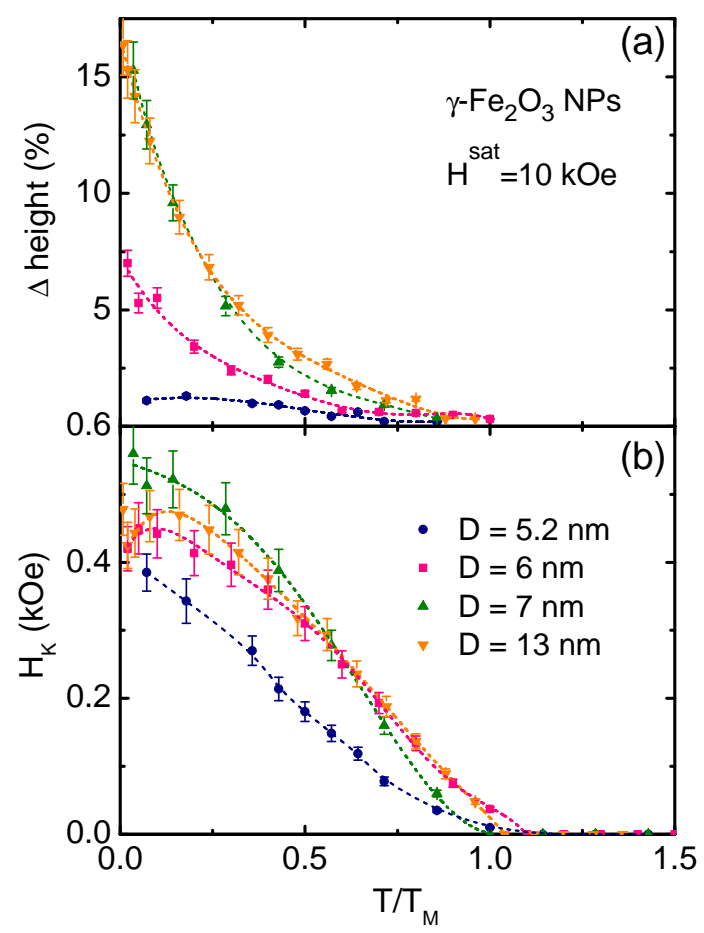

Figure 7.10: Comparison of (a) the anisotropy peak height difference and (b) $H_{\mathrm{K}}$ values extracted from the TS scans, both plotted as a function of $T / T_{M}$ in the four maghemite NPs samples. Dashed lines are guides to the eye.

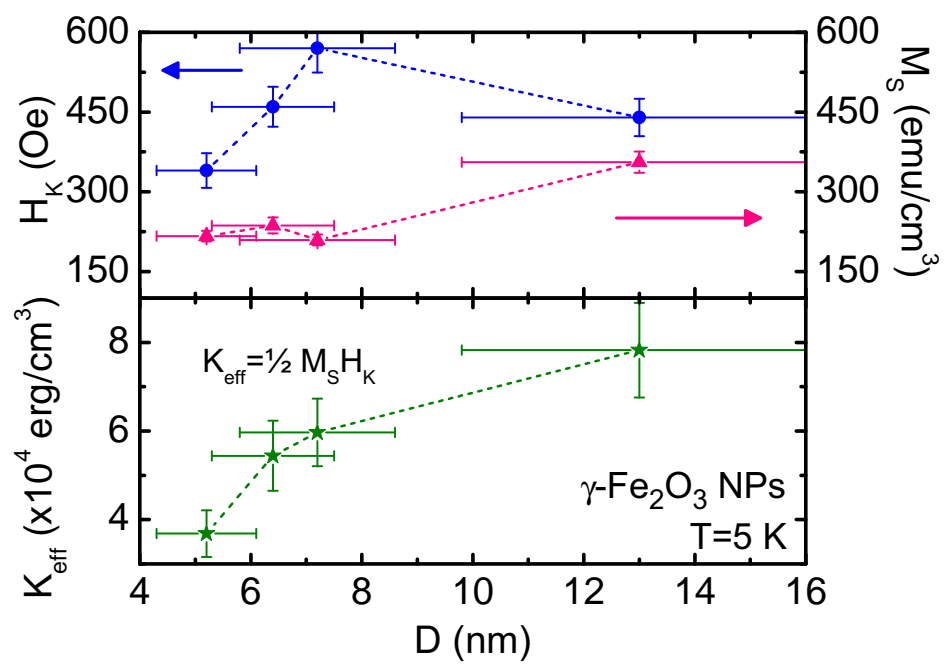

Figure 7.11: (top panel) $H_{\mathrm{K}}$ and $M_{\mathrm{S}}$ values as obtained from the TS scans and $\mathrm{M}(\mathrm{H})$ measurements, respectively. (bottom panel) $K_{\text {eff }}$ estimated from $H_{\mathrm{K}}$ and $M_{\mathrm{S}}$ values. The three parameters are plotted as a function of the particle diameter. 
tained from conventional SQUID magnetometry $\lfloor 207\rfloor$ and the values of $H_{\mathrm{K}}$ extracted from the TS measurements, as $K_{\text {eff }}=1 / 2 \quad M_{\mathrm{S}} H_{\mathrm{K}}$. The values of $H_{\mathrm{K}}$ and $M_{\mathrm{S}}$ for each sample, both at $T=5 \mathrm{~K}$, used to calculate $K_{\text {eff }}$ and the resulting values of the latter are given in table 7.2 and plotted in Fig. 7.11. $K_{\text {eff }}$ values are on the same order of those reported for the magnetocrystalline anisotropy in bulk maghemite $\left(2.0 \times 10^{4} \mathrm{erg} / \mathrm{cm}^{3}\right)\lfloor 212\rfloor . K_{\text {eff }}$ is found to increase as the particle diameter increases, which is consistent with the increase in the anisotropy as the core volume $\left(V_{\text {core }}\right)$ in the particles increases, as reported in Ref. $\lfloor 210\rfloor$. Comparing to those values obtained for the same maghemite NPs in Ref. [210」 (being of $7.7 \times 10^{5} \mathrm{erg} / \mathrm{cm}^{3}$ for the core contribu-

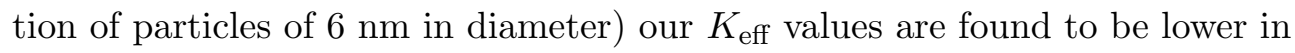
almost one order of magnitude. This difference may stem from the influence of the interparticle interactions in the position of $H_{\mathrm{K}}$ peaks in the TS scans, as it has been observed in similar NPs systems where the anisotropy field is found to increase with increasing interactions [47]. Indeed, we have demonstrated that our TS measurements are strongly influenced by inter-particle interactions, which may be affecting the correct estimation of $K_{\text {eff. }}$.

\subsubsection{Conclusions}

The increasing asymmetry in the peak heights in the TS scans of $\gamma-\mathrm{Fe}_{2} \mathrm{O}_{3}$ NPs samples is due to a combination of the anisotropy field distribution given by the size distribution in the samples, and the interparticle interactions that increase as the particle size increases. $K_{\text {eff values determined from the TS mea- }}$ surements for the particles are found to be close to those of bulk maghemite and increase as the particle diameter increases. These values, however, are lower than those previously determined for the same NPs systems in Ref. [210]. Such a difference may be due to the influence of interparticle interactions and anisotropy field distribution in the determination of $H_{\mathrm{K}}$ from TS measurements. 



\section{General Conclusions}

In this thesis we have performed an extensive study of different magnetic nanoparticles systems, elaborated by combination of diverse experimental techniques. We intended to center the attention in the capabilities of two characterization methods: the X-ray absorption spectroscopies (XANES, EXAFS and XMCD) and the $\mathrm{rf}$ transverse susceptibility. By using these techniques for electronic, structural and magnetic studies of nanoparticles systems, along with other complementary methods, it is possible to obtain a better understanding of the origin of their magnetic anisotropy, and many other interesting phenomena. The main findings for the magnetic nanoparticles studied here may be summarized as follows:

- Co-W NPs: we have described a system of self-organized amorphous Co-W alloy nanoparticles, uniformly dispersed in an alumina matrix. They show superparamagnetic behavior and are described as an array of non-interacting particles with random anisotropy axes and an average moment per particle proportional to the particle volume and to the average Co moment for each alloy composition. We prove that, despite their amorphous nature, values of the magnetic anisotropy constant deduced for Co-W alloyed NPs are on the order of $10^{6} \mathrm{erg} / \mathrm{cm}^{3}$, which is higher than that of bulk Co. Thus, we demonstrate that the magnetic anisotropy of amorphous alloy particles may be originated in mechanisms other than surface effects, such as structural short range order present in the $\mathrm{Co}-\mathrm{W}$ alloy.

Co-W hybridization and short range order identified in these Co-W NPs give rise to breakdown of Hund's third rule in the relative orientation of the induced orbital and spin moments in $\mathrm{W}$ atoms. Since such a breakdown is observed both experimentally and theoretically in nanoparticulate and bulk Co-W alloys, as well as in Fe/W films [120, our work suggests that the breakdown of this rule is a rather general trend in the case of $\mathrm{W}$ systems, taking place not only in metallic thin films, but also in nano-particulate and bulk materials. 
- Co-Pt NPs: these granular films are composed of particles with a pure cobalt core surrounded by an alloyed CoPt interface, embedded in a $\mathrm{Pt}$ matrix. Their magnetic properties are those of ferromagnetically coupled particles. We demonstrate that the main source of their magnetic anisotropy comes from the formation of Co-Pt alloy, which results in the presence of perpendicular magnetic anisotropy (PMA). The Co and $\mathrm{Pt}$ atoms in these particles are strongly magnetically coupled, as resulting from the alloy formation.

- Noble metal (Ag, Au, Pd, Pt) NPs: it is important to recall that without the element selective XMCD technique, this study of the $d$-band magnetism in noble metals particles would not have been possible, since it rules out any contribution from magnetic impurities other than the metals measured. Then, we have proven that the $d$-band magnetism in these NPs has an important orbital contribution as resulting of the asymmetry of the noble metal atoms coordination at the surface. This breaking of symmetry narrows the $d$ electron "surface band" and increases the number of $d$-holes with respect to their bulk counterpart.

- Magnetite $\left(\mathrm{Fe}_{3-\mathrm{x}} \mathrm{O}_{4}\right)$ NPs: radio frequency transverse susceptibility measurements in oleic acid- and $\mathrm{SiO}_{2}$-coated $\mathrm{Fe}_{3-\mathrm{x}} \mathrm{O}_{4}$ nanoparticles allowed a direct examination of the effects of the type of coating on their magnetic anisotropy, as a function of the particle size. On the one hand, $\mathrm{SiO}_{2}$ coating reduces the interparticle interactions as compared to oleic acid coating, the reduction being more effective for $5 \mathrm{~nm}$ than for $14 \mathrm{~nm}$ particles. On the other hand, $\mathrm{SiO}_{2}$ coating also decreases the magnetic anisotropy field measured on the particles. This is relevant concerning applications in biomedicine, since the $\mathrm{SiO}_{2}$ coating renders 5 and $14 \mathrm{~nm}$ hydrophilic particles with very limited agglomeration, low anisotropy, and superparamagnetic behavior at room temperature.

- Maghemite $\left(\gamma-\mathrm{Fe}_{2} \mathrm{O}_{3}\right)$ NPs: radio frequency magnetic transverse susceptibility measurements on $\gamma-\mathrm{Fe}_{2} \mathrm{O}_{3}$ nanoparticles allow to estimate their effective anisotropy constant, $K_{\text {eff. }}$ The resulting values range from 4 to 8 $\times 10^{4} \mathrm{erg} / \mathrm{cm}^{3}$, being on the order of the magnetocrystalline anisotropy in bulk maghemite. $K_{\text {eff }}$ values increase as the particle diameter increases. Evidences of anisotropy field distribution given by the size distribution in the samples, and interparticle interactions that increase as the particle size increases, are also observed in the TS measurements. 


\section{Perspectives}

We consider important to mention that there are still some open questions regarding the study of the NPs systems we describe in this dissertation. For example, in the case of Co-Pt nanoparticles, our investigation has to be continued by including the samples series with a fixed amount of $\mathrm{Pt}$ and variable Co thickness, in order to analyze their effects in their magnetic properties and PMA. Angle dependent XMCD measurements both at the Co and $\mathrm{Pt} L_{2,3}$ edges have been also performed on these Co-Pt particles in order to explore the evolution of the magnetization as a function of the sample and field orientation, with which we expect to obtain a better understanding of the PMA in these samples. We have also observed that the interactions between $\mathrm{Co}-\mathrm{Pt}$ NPs play an important role in their magnetic properties, then it would be important to quantify and evaluate them.

Another example is the Co-Pd NPs system introduced in Chapter 5, which shows PMA induced by alloying, just as the Co-Pt NPs case. In this system, the systematic analysis of the structural and magnetic properties is yet to be completed.

The study of noble metal NPs described in Chapter 5 might be complemented with results on assemblies of $\mathrm{Pd}$ NPs and $\mathrm{Pd}_{13}$ clusters in a zeolite (a system very similar to the $\mathrm{Pt}_{13}$ described in Chapter 5), which our group are currently working on. We have some projects and proposals in this direction, so that the respective XMCD measurements could be performed.

With respect to the transverse susceptibility measurements on NPs systems, we would like to deepen into the understanding of the anisotropy field dispersion and interparticle interactions observed in both magnetite and maghemite NPs, and their effects on the TS results. This may be achieved by simulations of the TS profiles following previous works, for example, the one by Matarranz et al. in Ref. 445 .

Finally, now that we have demonstrated the capabilities of the radio frequency (rf) transverse susceptibility technique (TS) developed for the facilities of the Universidad de Zaragoza, we shall make the most of it by continuing studying other interesting systems. Additionally, let us recall that this TS setup is now part of the Servicio de Medidas Físicas in the Universidad de Zaragoza, which means that many other users may benefit of it for performing their own research on magnetic anisotropy, spin dynamics, and short range correlations, to mention some of the possible topics to study with this equipment. 



\section{Bibliography}

[1] X. Batlle and A. Labarta, J. Phys. D Appl. Phys. 35, R15 (2002).

[2] J. Bansmann, S. H. Baker, C. Binns, J. A. Blackman, J. P. Bucher, J. D. Dávila, V. Dupuis, L. Favre, D. Kechrako, A. Kleibert, K. H. Meiwes-Broer, G. M. Pastor, A. Perez, O. Toulemonde, K. N. Trohidou, J. Tuaillon, and Y. Xie, Surf. Sci. Rep. 56, 189 (2005).

[3] N. A. Frey, S. Peng, K. Cheng, and S. Sun, Chem. Soc. Rev. 38, 2532 (2009).

[4] E. C. Stoner and E. P. Wolfarth, Phil. Trans. Roy. Soc. A240, 599 (1948).

[5] P. Tartaj, M. Morales, S. Veintemillas-Verdaguer, T. G.-C. no, and C. Serna, Journal of Physics D: Applied Physics 36, R182 (2003).

[6] T. Hayashi, S. Hirono, M. Tomita, and S. Umemura, Nature 381, 772 (1996).

[7] B. D. Terris and T. Thomson, J. Phys. D. Appl. Phys. 38, R199 (2005).

[8] J. Kim, S. Park, J. E. Lee, S. M. Jin, J. H. Lee, I. S. Lee, I. Yang, S. K. K. J.-S. Kim, M.-H. Cho, and T. Hyeon, Angew. Chem., Int. Ed. 45, 7754 (2006).

[9] G. F. Goya, V. Grazu, and M. R. Ibarra, Curr. Nanosci. 4, 1 (2008).

[10] C. Antoniak, J. Lindner, M. Spasova, D. Sudfeld, M. Acet, M. Farle, K. Fauth, U. Wiedwald, H.-G. Boyen, P. Ziemann, F. Wilhelm, A. Rogalev, and S. Sun, Phys. Rev. Lett. 97, 117201 (2006).

[11] J. Bartolomé, L. M. García, F. Bartolomé, F. Luis, R. López-Ruiz, F. Petroff, C. Deranlot, F. Wilhelm, A. Rogalev, P. Bencok, N. B. Brookes, L. Ruiz, and J. M. González-Calbet., Phys. Rev. B 77, 184420 (2008). 
[12] A. Fraile Rodríguez, A. Kleibert, J. Bansmann, A. Voitkans, L. J. Heyderman, and F. Nolting, Phys. Rev. Lett. 104, 127201 (2010).

[13] C. Antoniak, A. Warland, M. Darbandi, M. Spasova, A. Trunova, K. Fauth, E. F. Aziz, M. Farle, and H. Wende, J. Phys. D. Appl. Phys. 43, 474007 (2010).

[14] F. Jiménez-Villacorta, C. Prieto, Y. Huttel, N. D. Telling, and G. van der Laan, Phys. Rev. B 84, 172404 (2011).

[15] M. Niemeyer, K. Hirsch, V. Zamudio-Bayer, A. Langenberg, M. Vogel, M. Kossick, C. Ebrecht, K. Egashira, A. Terasaki, T. Möller, B. v. Issendorff, and J. T. Lau, Phys. Rev. Lett. 108, 057201 (2012).

[16] P. Bruno, Phys. Rev. B 39, 865 (1989).

[17] F. Luis, J. M. Torres, L. M. García, J. Bartolomé, J. Stankiewicz, F. Petroff, F. Fettar, J. L. Maurice, and A. Vaures, Phys. Rev. B 65, 094409 (2002).

[18] F. Luis, F. Petroff, J. M. Torres, L. M. García, J. Bartolomé, J. Carrey, and A. Vaurès, Phys. Rev. Lett. 88, 217205 (2002).

[19] F. Luis, F. Bartolomé, F. Petroff, J. Bartolomé, L. M. García, C. Deranlot, H. Jaffrès, M. J. Martínez, P. Bencok, F. Wilhelm, A. Rogalev, and N. Brookes, Europhys. Lett. 76, 142 (2006).

[20] J. Bartolomé, F. Luis, F. Petroff, F. Bartolomé, L. M. García, V. Cross, and H. Jaffres, Phys. Met. Metallogr. 99, S8 (2005).

[21] J. Bartolomé, F. Bartolomé, L. M. García, F. Luis, F. Petroff, V. Cros, H. Jaffrès, and A. Vaurès, "Magnetic dynamics of co nanospheres: Origin of the enhanced anisotropy," in Smart Materials for Ranging Systems, edited by J. Franse, V. Eremenko, and V. Sirenko (Springer, The Netherlands, 2006) pp. 1-25, arw nato no. 4 ed.

[22] F. Tournus, A. Tamion, N. Blanc, A. Hannour, L. Bardotti, B. Prével, P. Ohresser, E. Bonet, T. Epicier, and V. Dupuis, Phys. Rev. B 77, 144411 (2008).

[23] G. Hadjipanayis and P. Gaunt, J. Appl. Phys. 50, 2358 (1979).

[24] Q. F. Xiao, E. Bruck, Z. D. Zhang, F. R. de Boer, and K. H. J. Buschow, J. Alloys. Comp. 364, 64 (2004).

[25] K. H. J. Buschow, P. van Engen, and R. Jongebreur, J. Magn. Magn. Mat. 38, 1 (1983). 
[26] M. Naoe, H. Kazama, Y. Hoshi, and S. Yamanaka, J. Appl. Phys. 53, 7846 (1982).

[27] K. H. J. Buschow, J. Appl. Phys. 54, 2578 (1983).

[28] J. Dubowik, Y. V. Kudryavtsev, and R. Gontarz, Acta Phys. Pol. A. 76, 331 (1989).

[29] M. A. M. Ibrahim, S. S. A. E. Rehim, and S. O. Moussa, J. Appl. Electochem. 33, 627 (2003).

[30] J. Arbiol, F. Peiro, A. Cornet, C. Clavero, A. Cebollada, G. Armelles, and Y. Huttel, Appl. Phys. Lett. 86, 032510 (2005).

[31] A. Ebbing, O. Hellwig, L. Agudo, G. Eggeler, and O. Petracic, Phys. Rev. B 84, 012405 (2011).

[32] N. Nishimura, T. Hirai, A. Koganei, T. Ikeda, K. Okano, Y. Sekiguchi, and Y. Osada, J. Appl. Phys. 91, 5246 (2002).

[33] S. Ikeda, K. Miura, H. Yamamoto, K. Mizunuma, H. D. Gan, M. Endo, S. Kanai, J. Hayakawa, F. Matsukura, and H. Ohno, Nat. Mater. 9, 721-724 (2010).

[34] J. Bartolomé, F. Bartolomé, L. M. García, E. Roduner, Y. Akdogan, F. Wilhelm, and A. Rogalev, Phys. Rev. B 80, 014404 (2009).

[35] S. Selenska-Pobell, T. Reitz, R. Schönemann, T. Herrmannsdörfer, M. Marroun, A. Geissler, J. Bartolomé, F. Bartolomé, L. M. García, F. Wilhelm, and A. Rogalev, Nanomater. Nanotechnol. 1, 8 (2011).

[36] C. Leighton, D. D. Stauffer, Q. Huang, Y. Ren, S. El-Khatib, M. A. Torija, J. Wu, J. W. Lynn, L. Wang, N. A. Frey, H. Srikanth, J. E. Davies, K. Liu, and J. F. Mitchell, Phys. Rev. B 79, 214420 (2009).

[37] A. I. Figueroa, S. Chandra, M. H. Phan, H. Srikanth, C. M. Bonilla, L. M. García, F. Bartolomé, J. Bartolomé, and J. Herrero-Albillos, J. Appl. Phys. 109, 07E118 (2011).

[38] C. M. Bonilla, I. Calvo, J. Herrero-Albillos, A. I. Figueroa, C. CastanGuerrero, J. Bartolomé, J. A. Rodriguez-Velamazan, D. Schmitz, E. Weschke, D. Paudyal, V. K. Pecharsky, K. A. Gschneidner, Jr., F. Bartolomé, and L. M. García, J. Appl. Phys. 111, 07E315 (2012).

[39] G. T. Woods, P. Poddar, H. Srikanth, and Y. M. Mukovsk, J. Appl. Phys. 97, 10C104 (2005). 
[40] M. C. Contreras, J. F. Calleja, M. Rivas, M. O. Gutiérrez, and J. A. Corrales, J. Magn. Magn. Mat. 175, 64 (1997).

[41] N. A. Frey, S. Srinath, H. Srikanth, M. Varela, S. Pennycook, G. X. Miao, and A. Gupta, Phys. Rev. B 74, 024420 (2006).

[42] M. C. Contreras, J. F. Calleja, R. Matarranz, B. Presa, J. A. Corrales, and G. Pan, J. Appl. Phys. 99, 08F110 (2006).

[43] G. Ju, H. Zhou, R. Chantrell, B. Lu, and D. Weller, J. Appl. Phys. 99, 083902 (2006).

[44] L. Spinu, H. Srikanth, E. E. Carpenter, and C. J. O'Connor, J. Appl. Phys. 87, 5490 (2000).

[45] R. Matarranz, M. C. Contreras, G. Pan, B. Presa, J. A. Corrales, and J. F. Calleja, J. Appl. Phys. 99, 08 Q504 (2006).

[46] J. Soares, F. Machado, J. de Araújo, F. Cabral, H. Rodrigues, and M. Ginani, Phys. B 384, 85 (2006).

[47] P. Poddar, M. B. Morales, N. A. Frey, S. A. Morrison, E. E. Carpenter, and H. Srikanth, J. Appl. Phys. 104, 063901 (2008).

[48] N. A. Frey, Surface and interface magnetism in nanostructures and thin films, Ph.D. thesis, University of South Florida (2008).

[49] L. Pareti and G. Turilli, J. Appl. Phys. 61, 5098 (1987).

[50] A. Hoare, R. W. Chantrell, W. Schmitt, and A. Eiling, J. Phys. D: Appl. Phys. 26, 461 (1993).

[51] H. Srikanth, J. Wiggins, and H. Rees, Rev. Sci. Instrum. 70, 3097 (1999).

[52] R. C. Black and F. C. Wellstood, "Measurements of magnetism and magnetic properties of matter," in The SQUID Handbook: Applications of SQUIDs and SQUID Systems, Volume II, edited by J. Clarke and A. I. Braginski (John Wiley \& Sons, New York, 2006) Chap. 12, pp. 391-480.

[53] E. H. Hall, American Journal of Mathematics 2, pp. 287 (1879).

[54] A. Gerber, A. Milner, M. Karpovsky, B. Lemke, H.-U. Habermeier, J. Tuaillon-Combes, M. Négrier, O. Boisron, P. Mélinon, and A. Perez, J. Magn. Magn. Mater. 242-245, 90 (2002).

[55] J. Lindemuth and B. Dodrill, IEEE Trans. Magn. 40, 2191 (2004). 
[56] N. Nagaosa, J. Sinova, S. Onoda, A. H. MacDonald, and N. P. Ong, Rev. Mod. Phys. 82, 1539 (2010).

[57] J. Hollas, Modern Spectroscopy (John Wiley \& Sons, 2004).

[58] M. Hof, "Basics of optical spectroscopy," in Handbook of Spectroscopy (Wiley-VCH Verlag GmbH \& Co. KGaA, 2005) pp. 37-47.

[59] S. Conradson, Los Alamos Science 26, 422 (2000).

[60] M. Newville, (2004).

[61] A. Filipponi, M. Borowski, D. T. Bowron, S. Ansell, A. D. Cicco, S. D. Panfilis, and J. Itie, Rev. Sci. Intrum. 71, 2422 (2000).

[62] G. Schütz, M. Knülle, R. Wienke, W. Wilhelm, W. Wagner, P. Kienle, and R. Frahm, Z. Phys. B - Condensed Matter 73, 67 (1988).

[63] U. Fano, Phys. Rev. 178, 131 (1969).

[64] B. T. Thole, P. Carra, F. Sette, and G. vanderLaan, Phys. Rev. Lett. 68, 1943 (1992).

[65] P. Carra, B. T. Thole, M. Altarelli, and X. Wang, Phys. Rev. Lett. 70, 694 (1993).

[66] J. Stohr, J. Electron Spectrosc. Relat. Phenom. 75, 253 (1995).

[67] C. T. Chen, Y. U. Idzerda, H.-J. Lin, N. V. Smith, G. Meigs, E. Chaban, G. H. Ho, E. Pellegrin, and F. Sette, Phys. Rev. Lett. 75, 152 (1995).

[68] J. Stohr, Phys. Rev. Lett. 75, 3748 (1995).

[69] C. Piamonteze, P. Miedema, and F. M. F. de Groot, Phys. Rev. B 80, 184410 (2009).

[70] V. V. Krishnamurthy, D. J. Singh, N. Kawamura, M. Suzuki, and T. Ishikawa, Phys. Rev. B 74, 064411 (2006).

[71] M. W. Grinstaff, M. B. Salamon, and K. S. Suslick, Phys. Rev. B 48, 269 (1993).

[72] X. Cao, Y. Koltypin, G. Kataby, R. Prozorov, and A. Gedanken, J. Mater. Res. 10, 2952 (1995).

[73] X. Cao, Y. Koltypin, R. Prozorov, G. Katabya, and A. Gedanken, J. Mater. Chem. 7, 2447 (1997). 
[74] K. J. Carroll, J. A. Pitts, K. Zhang, A. K. Pradhan, and E. E. Carpenter, J. Appl. Phys. 107, 09A302 (2010).

[75] M. Muruganandham, R. Amutha, B. Ahmmad, E. Repo, and M. Sillanpaa, J. Phys. Chem. C 114, 22493 (2010).

[76] G. Kataby, A. Ulman, R. Prozorov, and A. Gedanken, Langmuir 14, 1512 (1998).

[77] G. Kataby, Y. Koltypin, A. Ulman, I. Felner, and A. Gedanken, Appl. Surf. Sci. 201, 191 (1997).

[78] J. Dormann, D. Fiorani, and E. Tronc, Adv. Chem. Phys. 98, 283 (1997).

[79] J. Dormann, A. Belayachi, J. Maknani, A. Ezzir, M. Cruz, M. Godinho, R. Cherkaoui, and M. Nogues, J. Magn. Magn. Mat. 185, 1 (1998).

[80] E. DeBiasi, C. A. Ramos, R. D. Zysler, and H. Romero, Phys. Rev. B 65, 144416 (2002).

[81] R. Zysler, H. Romero, C. Ramos, E. DeBiasi, and D. Fiorani, J. Magn. Magn. Mat. 266, 233 (2003).

[82] Y. Koltypin, G. Katabi, X. Cao, R. Prozorov, and A. Gedanken, J. Non-Cryst. Solids 201, 159 (1996).

[83] K. V. P. M. Shafi, A. Gedankenb, R. B. Goldfarb, and I. Felner, J. Appl. Phys. 81, 6901 (1997).

[84] K. V. P. M. Shafi, Y. Koltypin, A. Gedanken, R. Prozorov, J. Balogh, J. Lendvai, and I. Felner, J. Phys. Chem. B 101, 6409 (2010).

[85] M. Donten and Z. Stojek, J. Appl. Electochem. 26, 665 (1996).

[86] M. Donten, T. Gromulski, and Z. Stojek, J. Alloys. Comp. 279, 272 (1998).

[87] K. Ounadjela, G. Suran, and F. Machizaud, Phys. Rev. B 40, 578 (1989).

[88] M. Naili and G. Suran, J. Magn. Magn. Mat. 135, 141 (1994).

[89] D. Babonneau, F. Petroff, J. L. Maurice, F. Fettar, A. Vaures, and A. Naudons, Appl. Phys. Lett. 76, 2892 (2000).

[90] J. Rodriguez-Carvajal, Phys. B 192, 55 (1993). 
[91] T. Omi, H. Yamamoto, and H. L. Glass, J. Electrochem. Soc. 119, 168 (1972).

[92] F. Machizaud, K. Ounadjela, and G. Suran, Phys. Rev. B 40, 587 (1989).

[93] D. Babonneau, D. Lantiat, S. Camelio, J. Toudert, L. Simonot, F. Pailloux, M.-F. Denanot, and T. Girardeau, Eur. Phys. J. Appl. Phys. 44, 3 (2008).

[94] J. Briático, J.-L. Maurice, J. Carrey, D. Imhoff, F.Petroff, and A. Vaurès., Eur. Phys. J. D. 9, 517 (1999).

[95] G. Qin, N. Xiao, B. Yang, Y. Ren, W. Pei, and X. Zhao, Acta Metall. Sin. 22, 415 (2009).

[96] B. Ravel and M. Newville, J. Synchrotron Rad. 12, 537 (2005).

[97] B. Ravel, J. Synchrotron Rad. 8, 314 (2001).

[98] B. Moraweck, A. J. Renouprez, E. K. Hlil, and R. Baudoing-Savois, J. Phys. Chem. 97, 4288 (1993).

[99] M. Kuhn, T. Sham, J. Chen, and K. Tan, Sol. State Comm. 75, 861 (1990).

[100] A. Yevick and A. I. Frenkel, Phys. Rev. B 81, 115451 (2010).

[101] A. L. Ageev, Y. A. Babanov, V. V. Vasin, N. V. Ershov, and A. V. Serikov, Phys. Stat. Sol. (b) 117, 345 (1983).

[102] M. L. Fdez-Gubieda, A. García-Arribas, I. Orue, F. Plazaola, and J. M. Barandiarán, Europhys. Lett. 40, 43 (1997).

[103] A. Sadoc, D. Raoux, P. Lagarde, and A. Fontaine, J. Non-Cryst. Solids 50, 331 (1982).

[104] R. D. Zysler, E. DeBiasi, C. A. Ramos, D. Fiorani, and H. Romero, "Surface and interparticle effects in amorphous magnetic nanoparticles," in Surface Effects in Magnetic Nanoparticles, edited by D. Fiorani (Springer, USA, 2005) Chap. 8, pp. 239-261.

[105] P. Hansen, "Magnetic amorphous alloys," in Handbook of Magnetic Materials, Vol. 6, edited by K. Buschow (Elsevier Science Pub. B.V., The Netherlands, 1991) Chap. 4, pp. 289-452.

[106] P. Bracconi and L. C. Dufour, Metall. Mater. Trans. B 7, 321 (1976). 
[107] M. Tischer, O. Hjortstam, D. Arvanitis, J. H. Dunn, F. May, K. Baberschke, J. Trygg, J. M. Wills, B. Johansson, and O. Eriksson, Phys. Rev. Lett. 75, 1602 (1995).

[108] M. G. Samant, J. Stohr, S. S. P. Parkin, G. A. Held, B. D. Hermsmeier, F. Herman, M. van Schilfgaarde, L.-C. Duda, D. C. Mancini, N. Wassdahl, and R. Nakajima, Phys. Rev. Lett. 72, 1112 (1994).

[109] R. Nakajima, J. Stöhr, and Y. U. Idzerda, Phys. Rev. B 59, 6421 (1999).

[110] K. Fauth, Appl. Phys. Lett. 85, 3271 (2004).

[111] A. Kleibert, K.-H. Meiwes-Broer, and J. Bansmann, Phys. Rev. B 79, 125423 (2009).

[112] R. C. O'Handley, "Amorphous materials: Magnetism and disorder," in Modern magnetic materials: principles and applications, A Wileyinterscience publication (Wiley, 2000) Chap. 11, pp. 391-431.

[113] A. R. Mackintosh and O. K. Andersen, "The electronic structure of transition metals," in Electrons at the Fermi surface, edited by M. Springford (Cambridge University Press, 1980) Chap. 5, pp. 149-224.

[114] M. Vijayakumar and M. S. Gopinathan, J. Mol. Struct. 361, 15 (1996).

[115] W. A. Sucksmith and J. E. Thompson, Proc. R. Soc. London 225, 362 (1954).

[116] M. Spasova, U. Wiedwald, R. Ramchal, M. Farle, M. Jergel, E. Majkova, S. Luby, and R. Senderak, Phys. Stat. Sol. (b) 225, 449 (2001).

[117] M. Ohkoshi, N. Watanabe, and K. Tsushima, J. Magn. Magn. Mat. 113, 92 (1992).

[118] M. Rossignol, M. Schlenker, and Y. Samson, "Ferromagnetism of an ideal system," in Magnetism: Fundamentals, Magnetism, edited by É. Lacheisserie, D. Gignoux, and M. Schlenker (Springer, 2005) Chap. 5, pp. 143-208.

[119] J. A. Osborn, Phys. Rev. 67, 351 (1945).

[120] F. Wilhelm, P. Poulopoulos, H. Wende, A. Scherz, K. Baberschke, M. Angelakeris, N. K. Flevaris, and A. Rogalev, Phys. Rev. Lett. 87, 207202 (2001).

[121] F. Hund, Z. Phys. 33, 345 (1925). 
[122] J. Herrero-Albillos, L. M. García, F. Bartolomé, and A. T. Young, Europhys. Lett. 93, 17006 (2011).

[123] V. Kapaklis, P. T. Korelis, B. Hjörvarsson, A. Vlachos, I. Galanakis, P. Poulopoulos, K. Özdoğan, M. Angelakeris, F. Wilhelm, and A. Rogalev, Phys. Rev. B 84, 024411 (2011).

[124] Y. H. Matsuda, J. L. Her, S. Michimura, T. Inami, M. Suzuki, N. Kawamura, M. Mizumaki, K. Kindo, J. Yamauara, and Z. Hiroi, Phys. Rev. B 84, 174431 (2011).

[125] F. Wilhelm, N. Jaouen, A. Rogalev, W. G. Stirling, R. Springell, S. W. Zochowski, A. M. Beesley, S. D. Brown, M. F. Thomas, G. H. Lander, S. Langridge, R. C. C. Ward, and M. R. Wells, Phys. Rev. B 76, 024425 (2007).

[126] J. P. Rueff, R. M. Galéra, C. Giorgetti, E. Dartyge, C. Brouder, and M. Alouani, Phys. Rev. B 58, 12271 (1998).

[127] S. Pizzini, A. Fontaine, C. Giorgetti, E. Dartyge, J.-F. Bobo, M. Piecuch, and F. Baudelet, Phys. Rev. Lett. 74, 1470 (1995).

[128] B. L. Henke, E. M. Gullikson, and J. C. Davis, Atom. Data Nucl. Data 54, 181 (1993).

[129] A. N. Mansour, J. J. W. Cook, and D. E. Sayers, J. Phys. Chem. 88, 2330 (1984).

[130] I. Galanakis, M. Alouani, and H. Dreyssé, Phys. Rev. B 62, 3923 (2000).

[131] Y. Joly, Phys. Rev. B 63, 125120 (2001).

[132] O. Bunău and Y. Joly, J. Phys.: Condens. Matter 21, 345501 (2009).

[133] I. Galanakis, P. M. Oppeneer, P. Ravindran, L. Nordström, P. James, M. Alouani, H. Dreysse, and O. Eriksson, Phys. Rev. B 63, 172405 (2001).

[134] I. A. Campbell, J. Phys. F. Met. Phys. 2, L47 (1972).

[135] X. Qian and W. Hübner, Phys. Rev. B 67, 184414 (2003).

[136] R. Tyer, G. van der Laan, W. M. Temmerman, Z. Szotek, and H. Ebert, Phys. Rev. B 67, 104409 (2003).

[137] H. Wende, Rep. Prog. Phys. 67, 2105 (2004). 
[138] S. Rohart, C. Raufast, L. Favre, E. Bernstein, E. Bonet, and V. Dupuis, Phys. Rev. B 74, 104408 (2006)

[139] M. Maret, M. C. Cadeville, W. Staiger, E. Beaurepaire, R. Poinsot, and A. Herr, Thin Solid Films 275, 224 (1996).

[140] W. Grange, M. Maret, J. Kappler, J. Vogel, A. Fontaine, F. Petroff, and G. Krill, Phys. Rev. B 58, 6298 (1998).

[141] W. Grange, I. Galanakis, M. Alouani, M. Maret, J.-P. Kappler, and A. Rogalev, Phys. Rev. B 62, 1157 (2000).

[142] K. K. M. Pandey, J. S. Chen, T. Liu, C. J. Sun, and G. M. Chow, J. Phys. D: Appl. Phys. 42, 185007 (2009).

[143] Y. S. lee, J. Y. Rhee, C. N. Whang, and Y. P. Lee, Phys. Rev. B 68, 235111 (2003).

[144] J.-I. Park, M. G. Kim, Y. wook Jun, J. S. Lee, W. ram Lee, and J. Cheon, J. Am. Chem. Soc. 126, 9072 (2004).

[145] S. Mukerjee, S. Srinivasan, M. P. Soriaga, and J. McBreen, J. Electrochem. Soc. 142, 1409 (1995).

[146] M. Négrier, J. Tuaillon-Combes, V. Dupuis, and A. P. P. Mélinon, Philos. Mag. A 81, 2855 (2001).

[147] J. W. Knepper and F. Y. Yang, Phys. Rev. B 71, 224403 (2005).

[148] P. F. Carcia, J. Appl. Phys. 63, 5066 (1988).

[149] S. Hashimoto, Y. Ochiai, and K. Aso, J. Appl. Phys. 66, 4909 (1989).

[150] C. H. Lee, R. F. C. Farrow, C. J. Lin, E. E. Marinero, and C. J. Chien, Phys. Rev. B 42, 11384 (1990).

[151] N. Nakajima, T. Koide, T. Shidara, H. Miyauchi, H. Fukutani, A. Fujimori, K. Iio, T. Katayama, M. Nývlt, and Y. Suzuki, Phys. Rev. Lett. 81, 5229 (1998).

[152] J. Bartolomé, F. Luis, L. M. García, F. Bartolomé, F. Petroff, C. Deranlot, F. Wilhelm, A. Rogalev, P. Bencok, and N. B. Brookes, J. Magn. Magn. Mat. 316, e9 (2007).

[153] A. Gerber, A. Milner, J. Tuaillon-Combes, M. Négrier, O. Boisron, P. Mélinon, and A. Perez, J. Magn. Magn. Mater. 241, 340 (2002). 
[154] A. Maziewski, P. Mazalski, Z. Kurant, M. O. Liedke, J. McCord, J. Fassbender, J. Ferré, A. Mougin, A. Wawro, L. T. Baczewski, A. Rogalev, F. Wilhelm, and T. Gemming, Phys. Rev. B 85, 054427 (2012).

[155] P. Imperia, P. Andreazza, D. Schnitz, J. Penuelas, and C. AndreazzaVignole, J. Magn. Magn. Mat 310, 2417 (2007).

[156] P. Imperia, L. Glaser, M. Martins, P. Andreazza, J. Penuelas, V. Alessandrovic, H. Weller, C. Andreazza-Vignolle, and W. Wurth., Phys. Stat. Sol. (a) 205, 1047 (2008).

[157] E. C. Stoner, Proc. R. Soc. A 165, 372 (1938).

[158] M. Jamet, M. Négrier, V. Dupuis, J. Tuaillon-Combes, P. Mélinon, A. Pérez, W. Wernsdorfer, B. Barbara, and B. Baguenard, J. Magn. Magn. Mater. 237, 293 (2001).

[159] F. Bolzoni, F. Leccabue, R. Panizzieri, and L. Pareti, IEEE Trans. Magn. 20, 1625 (1984).

[160] M. E. Gruner, J. Phys.: Conf. Ser. 200, 072039 (2010).

[161] M. E. Gruner, J. Phys. D: Appl. Phys. 43, 474008 (2010).

[162] A. H. Morrish, "The magnetization of feromagnetic materials," in The physical properties of magnetism, Wiley series on the science and technology of materials (John Wiley \& sons, Inc., New York, 1965) Chap. 7, pp. $332-431$.

[163] M. Suzuki, N. Kawamura, H. Miyagawa, J. S. Garitaonandia, Y. Yamamoto, and H. Hori, Phys. Rev. Lett. 108, 047201 (2012).

[164] A. Bzowski, T. K. Sham, and Y. M. Yiu, Phys. Rev. B 49, 13776 (1994).

[165] J. Vogel, A. Fontaine, V. Cros, F. Petroff, J.-P. Kappler, G. Krill, A. Rogalev, and J. Goulon, Phys. Rev. B 55, 3663 (1997).

[166] A. Rogalev, F. Wilhelm, N. Jaouen, J. Goulon, and J.-P. Kappler, "X-ray magnetic circular dichroism: Historical perspective and recent highlights," in Magnetism: A Synchrotron Radiation Approach, Lecture Notes in Physics, Vol. 697, edited by E. Beaurepaire, H. Bulou, F. Scheurer, and J.-P. Kappler (Springer Berlin / Heidelberg, 2006) pp. 71-93.

[167] K. Adachi, D. Bonnenberg, J. J. M. Franse, R. Gersdorf, K. A. Hempel, K. Kanematsu, S. Misawa, M. Shiga, M. B. Stearns, and H. P. J. Wijn, Landolt-Börnstein, New series. Magnetic Properties of Metals: 3d, $4 d$ 
and 5d Elements, Alloys and Compounds, edited by H. P. J. Wijn, Vol. III/19a (Springer-Verlag, Berlin, 1986).

[168] L. Suber, D. Fiorani, G. Scavia, P. Imperatori, and W. R. Plunkett, Chem. Mater. 19, 1509 (2007).

[169] J. S. Garatonaindia, M. Insausti, E. Goikolea, M. Suzuki, J. D. Cashion, N. Kawamura, H. Ohsawa, I. Gil de Muro, K. Suzuki, F. Plazaola, and T. Rojo, Nanolett. 8, 375 (2008).

[170] Y. Yamamoto, T. Miura, M. Suzuki, N. Kawamura, H. Miyagawa, T. Nakamura, K. Kobayashi, T. Teranishi, and H. Hori, Phys. Rev. Lett. 93, 116801 (2004).

[171] J. de la Venta, E. F. Pinel, M. García, P. Crespo, A. Hernando, O. R. de la Fuente, A. Fernández, and S. Penadés, Mod. Phys. Lett. B 21, 303 (2007).

[172] J. Bartolomé, F. Bartolomé, L. M. García, A. I. Figueroa, A. Repollés, M. J. Martínez, F. Luis, C. Magén, S. Selenska-Pobell, F. Pobell, T. Reitz, R. Schönemann, T. Herrmannsdörfer, M. Merroun, A. Geissler, F. Wilhelm, and A. Rogalev, Phys. Rev. Lett. (Submitted for publication).

[173] T. Taniyama, E. Ohta, and T. Sato, Europhys. Lett. 38, 195 (1997).

[174] X. Liu, M. Bauer, H. Bertagnolli, E. Roduner, J. van Slageren, and F. Phillipp, Phys. Rev. Lett. 97, 253401 (2006).

[175] S. Trudel, Gold Bull. 44, 3 (2011).

[176] L. Spinu, C. J. O'Connor, and H. Srikanth, IEEE Trans. Magn. 37, 2188 (2001).

[177] A. Aharoni, E. H. Frei, S. Shtrikman, and D. Treves, Bull. Res. Counc. of Israel 6A, 215 (1957).

[178] P. A. Martínez and B. M. Monge, Int. J. Electron. 92, 619 (2005).

[179] J. M. García del Pozo, P. A. Martínez, A. Otín, J. P. Alegre, and D. Sancho, Int. J. Electron. 95, 1211 (2008).

[180] E. Gratz, R. Resel, A. T. Burkov, E. Bauer, A. S. Markosyan, and A. Galatanu, J. Phys.: Condens. Matter 7, 6687 (1995).

[181] J. Herrero-Albillos, F. Bartolomé, L. M. García, A. T. Young, T. Funk, J. Campo, and G. J. Cuello, Phys. Rev. B 76, 094409 (2007). 
[182] N. Pérez, F. Bartolomé, L. M. García, J. Bartolomé, M. P. Morales, C. J. Serna, A. Labarta, , and X. Batlle, Appl. Phys. Lett. 94, 093108 (2009).

[183] X. Batlle, N. Pérez, P. Guardia, O. Iglesias, A. Labarta, F. Bartolomé, L. M. García, J. Bartolomé, A. G. Roca, M. P. Morales, and C. J. Serna, J. Appl. Phys. 109, 07B524 (2011).

[184] J. Salafranca, J. Gazquez, N. Pérez, A. Labarta, S. T. Pantelides, S. J. Pennycook, X. Batlle, and M. Varela, Nano Lett. 12, 2499 (2012), http://pubs.acs.org/doi/pdf/10.1021/nl300665z .

[185] P. Guardia, B. Batlle-Brugal, A. Roca, O. Iglesias, M. Morales, C. Serna, A. Labarta, and X. Batlle, J. Magn. Magn. Mat. 316, e756 (2007).

[186] S. Pal, M. Morales, P. Mukherjee, and H. Srikanth, J. Appl. Phys. 105, 07B504 (2009).

[187] A. G. Roca, M. P. Morales, K. O’Grady, and C. J. Serna, Nanotechnology 17, 2783 (2006).

[188] P. Guardia, A. Labarta, and X. Batlle, J. Phys. Chem. C 115, 390 (2011).

[189] N. Perez, F. Lopez-Calahorra, A. Labarta, and X. Batlle, Phys. Chem. Chem. Phys. 13, 19485 (2011).

[190] R. Mejías, S. Pérez-Yagüe, A. G. Roca, N. Pérez, A. Villanueva, M. Canete, S. Manes, J. Ruiz-Cabello, M. Benito, A. Labarta, X. Batlle, S. Veintemillas-Verdaguer, M. P. Morales, D. F. Barber, and C. J. Serna, Nanomedicine UK 5, 397 (2010).

[191] B. Cullity, Introduction to magnetic materials, Addison-Wesley series in metallurgy and materials (Addison-Wesley Pub. Co., 1972).

[192] P. Guardia, N. Pérez, A. Labarta, and X. Batlle, Langmuir 26, $5843 \quad$ (2010), pMID: 20000725, http://pubs.acs.org/doi/pdf/10.1021/la903767e .

[193] P. Guardia, J. Perez-Juste, A. Labarta, X. Batlle, and L. M. Liz-Marzan, Chem. Commun. 46, 6108 (2010).

[194] J. Park, K. An, Y. Hwang, J.-G. Park, H.-J. Noh, J.-Y. Kim, J.-H. Park, N.-M. Hwang, and T. Hyeon, Nature Mater. 3, 891 (2004).

[195] D. C. Lee, F. V. Mikulec, J. M. Pelaez, B. Koo, and B. A. Korgel, J. Phys. Chem. B 110, 11160 (2006), http://pubs.acs.org/doi/pdf/10.1021/jp060974z . 
[196] F. J. Arriagada and K. Osseo-Asare, J. Colloid Interf. Sci. 211, 210 (1999).

[197] S. Yoon, J. Korean Phys. Soc. 59, 3069 (2011).

[198] P. Poddar, J. L. Wilson, H. Srikanth, D. F. Farrell, and S. A. Majetich, Phys. Rev. B 68, 214409 (2003).

[199] H. Pfeiffer, Phys. Status Solidi (a) 118, 295 (1990).

[200] C. de Julián Fernández, Phys. Rev. B 72, 054438 (2005).

[201] H. Pfeiffer, Phys. Status Solidi (a) 120, 233 (1990).

[202] H. Pfeiffer and W. Schüppel, Phys. Status Solidi (a) 119, 259 (1990).

[203] X. Batlle, M. Garcia del Muro, J. Tejada, H. Pfeiffer, P. Gornert, and E. Sinn, Journal of Applied Physics 74, 3333 (1993).

[204] P. M. Sollis, P. R. Bissell, and Y. Matsutake, IEEE Trans. Magn. 33, 3046 (1997).

[205] G. F. Goya, T. S. Berquó, F. C. Fonseca, and M. P. Morales, J. Appl. Phys. 94, 3520 (2003).

[206] N. Pérez, P. Guardia, A. G. Roca, M. P. Morales, C. J. Serna, O. Iglesias, F. Bartolomé, L. M. García, X. Batlle, and A. Labarta, Nanotechnology 19, 475704 (2008).

[207] A. Urtizberea, Open problems in the magnetic behavior of iron-oxide nanoparticles, Ph.D. thesis, Universidad de Zaragoza (2011).

[208] B. Martínez, X. Obradors, L. Balcells, A. Rouanet, and C. Monty, Phys. Rev. Lett. 80, 181 (1998).

[209] A. Millán, A. Urtizberea, N. J. O. Silva, F. Palacio, V. S. Amaral, E. Snoeck, and V. Serin, J. Magn. Magn. Mat. 312, L5 (2007).

[210] Y. Komorida, M. Mito, H. Deguchi, S. Takagi, A. Millán, N. J. O. Silva, and F. Palacio, Appl. Phys. Lett. 94, 202503 (2009).

[211] J. L. Dormann, F. D’Orazio, F. Lucari, E. Tronc, P. Prené, J. P. Jolivet, D. Fiorani, R. Cherkaoui, and M. Noguès, Phys. Rev. B 53, 14291 (1996).

[212] E. P. Valstyn, J. P. Hanton, and A. H. Morrish, Phys. Rev. 128, 2078 (1962). 


\section{Publications}

1. A. I. Figueroa, F. Bartolomé, J. Bartolomé, L. M. García, F. Petroff, C. Deranlot, F. Wilhelm and A. Rogalev. Breakdown of Hund's third rule in amorphous $\mathrm{Co}-\mathrm{W}$ nanoparticles and crystalline $\mathrm{Co}_{3} \mathrm{~W}$ alloys. Physical Review B. 86(6):064428. 2012. doi:10.1103/PhysRevB.86.064428

2. J. Bartolomé, A. I. Figueroa, L. M. García, F. Bartolomé, L. Ruiz, J. M. González-Calbet, F. Petroff, C. Deranlot, F. Wilhelm, A. Rogalev and N. Brookes. Perpendicular magnetic anisotropy in Co-Pt granular multilayers. Low Temperature Physics/Fizika Nizkikh Temperatur (Special issue: Prof. Eremenko's 80th anniversary honor book - Kharkov). 38(9):1053. 2012.

3. A. I. Figueroa, J. Bartolomé, J. M. García del Pozo, A. Arauzo, E. Guerrero, P. Téllez, F. Bartolomé and L. M. García. Low temperature radio-frequency transverse susceptibility measurements using a CMOS oscillator circuit. Journal of Magnetism and Magnetic Materials. 324(17):2669-2675. 2012. doi:10.1016/j.jmmm.2012.03.058

4. C. M. Bonilla, I. Calvo, J. Herrero-Albillos, A. I. Figueroa, C. CastánGuerrero, J. Bartolomé, J. A. Rodriguez-Velamazan, D. Schmitz, E. Weschke, D. Paudyal, V. K. Pecharsky, K. A. Gschneidner, Jr., F. Bartolomé, L. M. García. New magnetic configuration in paramagnetic phase of $\mathrm{HoCo}_{2}$. Journal of Applied Physics, 111:07E315. 2012. doi:10.1063/1.3672258

5. S. Chandra, A. I. Figueroa, Barnali Ghosh, A. K. Raychaudhuri, M. H. Phan, P. Mukherjee and H. Srikanth. Fabrication and magnetic response probed by RF transverse susceptibility in $\mathrm{La}_{0.67} \mathrm{Ca}_{0.33} \mathrm{MnO}_{3}$ nanowires. Physica B, 407(1):175-178. 2012. doi:10.1016/j.physb.2011.10.021 
6. A. I. Figueroa, J. Bartolomé, L. M. García, F. Bartolomé, C. Magén, A. Ibarra, L. Ruiz, J. M. González-Calbet, F. Petroff, C. Deranlot, S. Pascarelli, P. Bencok, N. B. Brookes, F. Wilhelm and A. Rogalev. Structural and magnetic properties of amorphous Co-W alloyed nanoparticles. Physical Review B, 84(18):184423. 2011.

doi:10.1103/PhysRevB.84.184423

7. S. Chandra, A. I. Figueroa, Barnali Ghosh, M. H. Phan, H. Srikanth, and A. K. Raychaudhuri. Phase coexistence and magnetic anisotropy in polycrystalline and nanocrystalline $\mathrm{LaMnO}_{3+\delta}$. Journal of Applied Physics, 109:07D720. 2011. doi:10.1063/1.3551734

8. A. I. Figueroa, S. Chandra, M. H. Phan, H. Srikanth, C. M. Bonilla, L. M. García, F. Bartolomé, J. Bartolomé and J. Herrero-Albillos. Magnetic switching and magnetic transitions in $\mathrm{ErCo}_{2}$ probed by radiofrequency transverse susceptibility. Journal of Applied Physics, 109:07E118. 2011. doi:10.1063/1.3553936

9. N. B. Ivanova, J. Bartolomé, A. Figueroa, J. Blasco, A. Arauzo, M. S. Platunov, V. V. Rudenko and N. V. Kazak. The Influence of Ca Substitution on Magnetic and Electric Properties of $\mathrm{GdCoO}_{3-\delta}$ Cobaltite. Solid State Phenomena, 168-169:501-504. 2011. doi:10.4028/www.scientific.net/SSP.168-169.501

10. A. I. Figueroa, J. Bartolomé, L. M. García, F. Bartolomé, C. Magén, A. Ibarra, L. Ruiz, J. M. González-Calbet, F. Petroff and C. Deranlot. Morphology and magnetic properties of W-capped Co nanoparticles. Journal of Applied Physics, 107:09B508. 2010. doi:10.1063/1.3368725

11. A. Martínez, J. Spottorno, A. I. Figueroa, F. Bartolomé, L. M. García, C. Prestipino, A. Hernando and P. Crespo. Direct measurements of the correlation between reentrant ferromagnetism and lattice expansion in FeCuZr alloys. Physical Review B, 82(1):012406. 2010.

doi:10.1103/PhysRevB.82.012406

12. A. Martínez, A. I. Figueroa, F. Bartolomé, L. M. García, C. Prestipino, A. Hernando and P. Crespo. Correlation between anomalous temperature thermoremanence dependence measurements and thermal dilation in FeCuZr alloys. Journal of alloys and compounds. 2012. Accepted.

13. J. Bartolomé, A. I. Figueroa, F. Bartolomé, L. M. García, F. Wilhelm and A. Rogalev. d-band magnetism of $\mathrm{Ag}, \mathrm{Au}, \mathrm{Pd}$ and $\mathrm{Pt}$ studied with XMCD. Solid State Phenomena. 2012. Accepted. 
14. J. Bartolomé, F. Bartolomé, L. M. García, A. I. Figueroa, A. Repollés, M. J. Martínez, F. Luis, C. Magén, S. Selenska-Pobell, F. Pobell, T. Reitz, R. Schönemann, T. Herrmannsdörfer, M. Merroun, A. Geissler, F. Wilhelm, and A. Rogalev. Strong paramagnetism of Au nanoparticles deposited on a Sulfolobus acidocaldarius S-layer. Physical Review Letters. 2012. Submitted.

15. A. I. Figueroa, C. Moya, J. Bartolomé, F. Bartolomé, L. M. García, N. Pérez, A. Labarta, X. Batlle. $\mathrm{SiO}_{2}$ coating effects in the magnetic anisotropy of $\mathrm{Fe}_{3-x} \mathrm{O}_{4}$ nanoparticles suitable for bio-applications. Nanotechnology. 2012. Submitted. 
ISBN 978-84-15770-49-7

$9\left\|_{788415}\right\|_{770497}$

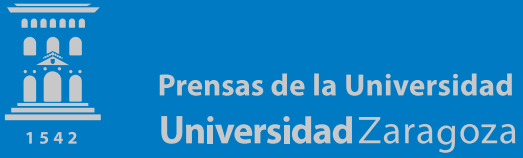

